V693

\section{The combined status of ATM and p53 links tumor development and therapeutic response}

\section{Jiang, H. ${ }^{1}$; Yaffe, M.B. ${ }^{1}$; Hemann, M. ${ }^{1}$; Reinhardt, H.C. ${ }^{2}$}

${ }^{1}$ Massachusetts Institute of Technology, Koch Institute for Integrative Cancer Research, Cambridge, United States, ${ }^{2}$ Uniklinik Köln, Medizinische Klinik 1, Köln, Germany

While the contribution of specific tumor suppressor networks to cancer development has been the subject of considerable recent study, it remains unclear how alterations in these networks integrate to influence the response of tumors to anti-cancer therapy. Here, we show that mechanisms commonly used by tumors to bypass early neoplastic checkpoints ultimately determine chemotherapeutic response and generate tumor-specific vulnerabilities that can be exploited with targeted therapies. Specifically, evaluation of the combined status of ATM and p53, two commonly mutated tumor suppressor genes, is sufficient to predict the clinical response to genotoxic cancer therapies. Using cell culture, mouse models and human tumor samples, we show that in p53 deficient settings, suppression of ATM dramatically sensitizes tumors to DNA damaging chemotherapy,due to the premature collapse of cell cycle checkpoints. Conversely, in the presence of functional p53, suppression of ATM or its downstream target Chk2, actually protects tumors from genotoxic therapy. This loss of ATM in p53-proficient cells selectively blunted the proapoptotic p53 response by preventing the induction of genes, such as Puma and Noxa However, the induction of protective cell cycle regulatory p53 target genes, such as p21 and Gadd45alpha, remained intact in this setting. Furthermore, we document that ATM-deficient cancer cells that contain wildtype p53 display strong non-oncogene addiction to DNA-PKcs for survival after DNA damage, such that suppression of DNA-PKcs in vivo resensitizes inherently chemoresistant ATM-deficient tumors to genotoxic chemotherapy. Thus, the specific set of alterations induced during tumor development plays a dominant role in determining both the response to conventional chemotherapy as well as the set of specific susceptibilities for targeted therapies in a given malignancy.

Disclosure: No conflict of interest disclosed.

\section{Freie Vorträge}

\section{Extranodale/T-Zell Lymphome}

\section{V694}

Identification of a novel Major Breakpoint Region (MBR) on the MALT1 gene from $\mathrm{t}(14 ; 18)(\mathrm{q} 32 ; \mathrm{q} 21) / / G H-M A L T 1-$ positive MALT lymphomas

Murga Penas, E.M. ${ }^{1}$; Callet-Bauchu, E. ${ }^{2}$; Ye, H. ${ }^{3}$; Gazzo, S. ${ }^{2}$; Berger, F. ${ }^{2}$; Schilling, G. ${ }^{1}$; Albert-Konetzny, N. ${ }^{1}$; Vettorazzi, E. Salles, G. ${ }^{2}$; Wlodarska, I. ${ }^{5}$, Du, M.-Q. ${ }^{3}$; Bokemeyer, C. ${ }^{1}$; Dierlamm, J. ${ }^{1}$

${ }^{1}$ University Medical Center Hamburg-Eppendorf, Department of Oncology and Hematology, BMT with section of Pneumology, Hubertus Wald Tumorzentrum-University Cancer Center Hamburg, Hamburg, Germany, ${ }^{2}$ Centre Hospitalier Lyon Sud, Department of Pathology and Hematology, Lyon, France, ${ }^{3}$ University of Cambridge, Department of Pathology, Cambridge, United Kingdom, ${ }^{4}$ University Medical Center HamburgEppendorf, Department of Medical Biometry and Epidemiology, Hamburg, Germany, ${ }^{5}$ University of Leuven, Center for Human Genetics and Flanders Interuniversity Institute for Biotechnology, Leuven, Belgium

Introduction: The $\mathrm{t}(14 ; 18)(\mathrm{q} 32 ; \mathrm{q} 21)$ involving the $I G H$ and the $M A L T 1$ genes is a recurrent abnormality in mucosa-associated lymphoid tissue (MALT) lymphomas. However, the nucleotide sequence of only one $\mathrm{t}(14 ; 18) / I G H$ $M A L T 1$-positive MALT lymphoma has been reported so far. We herein report the molecular genetic characterization of the direct MALT1-JH and reciprocal DH-MALT1 junctions in 5 new MALT lymphomas harboring the $\mathrm{t}(14 ; 18) /$ IGH-MALT1.

Methods: Five cases with the diagnosis of a MALT lymphoma, the presence of the $\mathrm{t}(14 ; 18) / I G H-M A L T 1$ as shown by interphase fluorescence in situ hybridization (FISH), and available frozen or paraffin-embedded tissue were collected. The direct IGH-MALTI fusions (MALT1-JH) were confirmed in 5 cases and the reciprocal IGH-MALTI fusion products $(D H-M A L T I)$ in 2 cases. The products were amplified and sequenced twice in both directions
Results: Similar to the $I G H$-associated translocations in follicular and mantle cell lymphomas, the IGH-MALT1 junctions in MALT lymphoma showed all features of a recombination signal sequence-guided V(D)J-mediated translocation at the $I G H$ locus. Furthermore, analogous to follicular and mantle cell lymphoma, templated nucleotides (T-nucleotides) were identified at the $\mathrm{t}(14 ; 18) / I G H-M A L T 1$ breakpoint junctions. On chromosome 18 , we identified a novel major breakpoint region in MALT1 upstream of its coding region. Moreover, the presence of duplications of MALT1 nucleotides in one case suggests an underlying staggered DNA-break process not consistent with V(D) $\mathrm{J}$-mediated recombination.

Conclusions: Our observations indicate that the pathomechanism underlying the $\mathrm{t}(14 ; 18) / I G H-M A L T 1$ in MALT lymphomas is probably based on an illegitimate $\mathrm{V}(\mathrm{D}) \mathrm{J}$-mediated recombination at the $I G H$ locus on chromosome 14 and a staggered double-stranded DNA break at the MALT1 locus on chromosome 18. Furthermore, we could identify a putative major breakpoint region (MBR) proximal to the 5' non-coding region of MALT1. The molecular characteristics of the $\mathrm{t}(14 ; 18) / I G H-M A L T 1$ resemble those found in the $\mathrm{t}(14 ; 18) /$ $I G H-B C L 2$ in follicular lymphoma and $\mathrm{t}(11 ; 14) / C C N D 1-I G H$ in mantle cell lymphoma, suggesting that these translocations could be generated by a common pathomechanism involving new synthesis of T-nucleotides and nonhomologous end joining (NHEJ) or alternative NHEJ repair pathways on the $I G H$-translocation partner.

Disclosure: No conflict of interest disclosed.

\section{V695}

\section{Temsirolimus for relapsed primary central nervous} system lymphoma: efficacy and pharmakokinetic data

\section{Kiewe, P. ${ }^{1}$; Korfel, A. ${ }^{1}$; Grobosch, T. $^{2}$; Thiel, E.}

${ }^{1}$ Charité Universitätsmedizin Berlin, Campus Benjamin Franklin, Hämatologie, Onkologie und Transfusionsmedizin, Berlin, Germany, ${ }^{2}$ Berliner Betrieb für Zentrale Gesundheitliche Aufgaben, Klinische Toxikologie und Pharmakologie, Berlin, Germany

Introduction: Salvage treatment in primary CNS lymphoma (PCNSL) after initial high-dose methotrexate (HDMTX) containing chemotherapy is poorly defined. Temsirolimus is a selective inhibitor of cell proliferation promoting intracellular protein mTOR with proven activity in lymphatic malignancies. We designed a phase II study (NCT00942747) to evaluate activity and toxicity of a temisrolimus monotherapy in relapsed PCNSL and to study its penetration into cerebrospinal fluid (CSF).

Methods: Included are immunocompetent patients with histologically confirmed PCNSL relapsing or progressive after HDMTX, age $\geq 18$ years, ECOG PS $\leq 2$, and adequate hepatic, renal and bone marrow function. Ten patients are planned to be treated with temsirolimus $25 \mathrm{mg}$ (cohort 1) and $75 \mathrm{mg}$ (cohort 2) once weekly. Primary endpoint is the overall response rate, secondary endpoints are toxicity, time to progression and CSF penetration of temsirolimus and its metabolite sirolimus. For CSF studies blood and CSF samples are simultaneously collected 30-60min after first, second and fourth infusion, and concentrations of temsirolimus and sirolimus are measured by HPLC with mass spectrometry detection.

Results: Five patients (median age 57 years, median ECOG PS 2) have been included and treated with $25 \mathrm{mg}$ Temsirolimus thus far. Median number of previous therapy regimens was 3 including whole brain irradiation in 3 patients. Patients received a median of 6 temsirolimus infusions. After 4 infusions, one patient had a partial remission (PR), three patients had stable disease (SD) (with tumor volume reductions of $32 \%-45 \%$ ), and one patient progressed. All patients with SD progressed after 2-4 further infusions. CTC zgrade III toxicity was thrombocytopenia in 1 patient and pneumonia in two patients. Ten blood/CSF pairs were collected in 5 patients. Mean maximum blood concentration was $292 \mathrm{ng} / \mathrm{ml}$ for temsirolimus and $37.2 \mathrm{ng} / \mathrm{ml}$ for sirolimus. No drug was detected in CSF (lower detection level $1 \mathrm{ng} / \mathrm{ml}$ ).

Conclusions: Temsirolimus $25 \mathrm{mg} /$ week showed some activity in the first 5 patients treated. Neither temsirolimus nor sirolimus could be found in CSF at this dose level. The study will continue at the $75 \mathrm{mg}$ dose level.

Disclosure: Philipp Kiewe: Honoraria: Wyeth/Pfizer Oncology; Financing of Scientific Research: Wyeth/Pfizer Oncology

Eckhard Thiel: Honoraria: Wyeth/Pfizer Oncology; Financing of Scientific Research: Wyeth/Pfizer Oncology 
V696

\section{Primary pulmonary lymphoma with an initial suspicion of a neuroendocrine tumor}

Kender, Z. ; Kunos, L. ${ }^{2}$; Losonczy, G. ${ }^{2}$; Sréter, L. '; Tóth, M.1; Racz, $K^{1}{ }^{1}$

${ }^{1} I I$. Department of Internal Medicine, Semmelweis University Faculty of Medicine, Budapest, Hungary, ${ }^{2}$ Department of Pulmonology, Semmelweis University Faculty of Medicine, Budapest, Hungary

We report a case of a primary pulmonary lymphoma mimicking a neuroendocrine tumor of the lung.

In 2009, a 62-year-old man with a history of hepatitis, renal stone and cutaneous basalioma has been examined because of fatigue. A round shadow in the right lung on a routine chest X-ray was already known since 2000, but the patient refused any further investigations at that time. Control chest X-ray in 2009 has revealed a moderate increase in the size of the right pulmonary lesion, furthermore a smaller lesion in the left lung has also been described Chest computed tomography has confirmed these solid lesions. Bronchoscopy has been negative.

Based on the slow progression of the pulmonary lesion, the possibility of a neuroendocrine tumor has been raised and somatostatin receptor scintigraphy has been performed. Both lesions turned out to be positive by scintigraphy. In contrast with the imaging results suggestive of a neuroendocrine tumor endocrinological investigation including serum chromogranin-A, serum neuron specific enolase, urinary 5-hydroxy-indol acetatic acid, metanephrin and normetanephrin levels have all been in the normal range.

Core needle aspiration biopsy has been successful at the second trial Preliminary histological examination based on hematoxilin-eosine staining suggested a neuroendocrine tumor, however, following detailed immunohis tochemical analysis this initial diagnosis has been withdrawn and the diagnosis of a CD20-positive marginal zone type non-Hodgkin lymphoma has been established.

PET-CT has not shown any extrapulmonry manifestations. The patient is currently under chemotherapy according to R-CHOP protocol.

In conclusion, this case presents the diagnostic difficulties related to a primary pulmonary non-Hodgkin lymphoma that mimicked a neuroendocrine tumor both by its appearance on imaging studies and even by its histological appearance. Careful endocrinological and histological analysis were needed to establish the diagnosis.

Disclosure: No conflict of interest disclosed.

\section{V697}

\section{ITK-SYK induces a T-cell lymphoproliferative disease in mice mimicking human disease}

\author{
Maurer, H. ${ }^{1}$; Sprissler, C. ${ }^{1}$; Fisch, P. ${ }^{1}$; Zirlik, K. ${ }^{1}$; Veelken, H. ${ }^{1}$; \\ Dierks, C. 1 \\ ${ }^{1}$ Uniklinik Freiburg, Hämatologie/Onkologie, Freiburg, Germany
}

Background: Peripheral T-cell lymphomas (PTCLs) display a major treatment problem with a high mortality rate due to the minimal effectiveness of conventional chemotherapy. Recent findings describe ITK-SYK as the first fusion oncogene identified in $17 \%$ of unspecified peripheral T-cell lymphomas and demonstrate overexpression of Syk in more than $90 \%$ of PTCLs.

Principle findings: In our experiments, we demonstrate that the expression of ITK-SYK in the bone marrow of Balbc mice induces a differentiation block in T-cell development and causes T-cell lymphomas in all transplanted mice within 8 weeks after transplantation. T-cell lymphomas were characterized by infiltration of spleen, lymph nodes, bone marrow and the skin with CD3+CD4+CD8- and CD3+CD4-CD8- malignant T-cells accompanied by a systemic inflammatory reaction with upregulation of IL-5 and Interferon- $\gamma$ (INF- $\gamma$ ). The disease was serially transplantable, inducing a clonal disease in secondary recipients. Malignant T-cells demonstrated enhanced apoptosis resistance and INF- $\gamma$ production in vitro. The action of ITK-SYK in vivo was dependent on the Syk kinase activity and the disease development could be inhibited by treatment of mice with the Syk inhibitor Curcumine ${ }^{7}$. In addition, alterations of the membrane binding of ITK-SYK or its interaction with the ubiquitin ligase $\mathrm{Cbl}$ by point mutations in the pleckstrin homology domain of Itk altered the transforming activity of ITK-SYK.
Conclusion: Our results demonstrate that ITK-SYK causes T-cell lymphomas in mice which mimic the human disease. Therefore, pharmacological inhibition of Syk in patients with PTCLs carrying the ITK-SYK fusion protein or other Syk fusions might be a new and effective treatment strategy.

Disclosure: No conflict of interest disclosed.

\section{V698}

\section{The fusion kinase ITK-SYK mimics a T cell receptor signal and drives oncogenesis in conditional mouse models of peripheral T cell lymphoma}

\section{Pechloff, K. ${ }^{1}$; Holch, J. ${ }^{1}$; Ferch, U. ${ }^{1}$; Schweneker, M. ${ }^{1}$; Brunner, K. ${ }^{1}$} Kremer, M.2; Sparwasser, T.3; Quintanilla-Martinez, L..,5. Zimber-Strobl, U. ${ }^{6}$; Streubel, B. ${ }^{7}$; Gewies, A. ${ }^{1,8}$; Peschel, C. ${ }^{1}$; Ruland, J. ${ }^{1,8}$

${ }^{1}$ Klinikum rechts der Isar, Technische Universität München, III.Med. Klinik, München, Germany, ${ }^{2}$ Institut für Pathologie, Technische Universität München, München, Germany, ${ }^{3}$ Institut für Medizinische Mikrobiologie, Immunologie und Hygiene, Technische Universität München, München, Germany, ${ }^{4}$ Institut für Pathologie, Helmholtz Zentrum München, Neuherberg, Germany, ${ }^{5}$ Institut für Pathologie, Eberhard Karls Universität Tübingen, Tübingen, Germany, ${ }^{6}$ Abteilung Genvektoren, Helmholtz Zentrum München, München, Germany, ${ }^{7} \mathrm{Klinisches} \mathrm{Institut} \mathrm{für} \mathrm{Pathologie,}$ Medizinische Universität Wien, Wien, Austria, ${ }^{8}$ Labor für Signaltransduktion im Immunsystem, Helmholtz Zentrum München, Neuherberg, Germany

Peripheral T cell lymphomas (PTCLs) belong to the category of the most aggressive non-Hodgkin's lymphomas with a high mortality rate, minimal effectiveness of conventional chemotherapy and only 10 - 30\% long-term survival. The molecular pathogenesis of this heterogenous group of lymphomas is not well understood and pre-clinical animal models for the disease are lacking. Recently, the chromosomal translocation $\mathrm{t}(5 ; 9)(\mathrm{q} 33 ; \mathrm{q} 22)$ was identified as a recurrent and specific genomic alteration in PTCL-unspecified. This translocation fuses the $S Y K$ (spleen tyrosine kinase) to the ITK (interleukin-2 (IL-2)-inducible T-cell kinase) gene, leading to the expression of the novel fusion tyrosine kinase ITK-SYK. Both, the ITK and the SYK kinase are required for normal antigen induced lymphocyte activation, however, the molecular and cellular consequences of ITK-SYK expression in lymphocytes are unknown.

By combining in vitro analysis with the generation of novel conditional mouse models expressing the fusion kinase ITK-SYK in a cell-type specific manner, we could show that ITK-SYK associates constitutively with lipid rafts in T cells and triggers antigen-independent phosphorylation of $\mathrm{T}$ cell receptor (TCR) proximal proteins, such as SLP-76, LAT and PLC-gamma1. These events lead to constitutive activation of the downstream AKT, ERK and p38 MAP kinase pathways and induce acute cellular outcomes that correspond to regular TCR ligation, including upregulation of CD69 and production of Interleukin-2 in vitro or deletion of thymocytes and activation of peripheral $\mathrm{T}$ cells in vivo. Ultimately, conditional expression of ITK-SYK in mice induces highly malignant PTCLs with $100 \%$ penetrance and clinical and pathological features resembling the human disease.

Taken together, our results show that ITK-SYK constitutively engages the antigen receptor signaling machinery in $\mathrm{T}$ cells, eventually culminating in the development of malignant PTCLs. We therefore provide in vivo evidence that constitutively enforced antigen receptor signaling can, in principle, act as a powerful oncogenic driver in lymphoma pathogenesis. Moreover, we establish a robust, clinically relevant and genetically tractable model of human PTCLs. Our novel mouse model will serve as a valuable tool for identifying and verifying compounds that could be of potential use for future treatment strategies for PTCL.

Disclosure: No conflict of interest disclosed. 
V699

\section{Role of miRNAs in $\mathrm{ALK}^{+}$and ALK' anaplastic large cell lymphoma}

\author{
Hamacher, F. ${ }^{1}$; Laimer, D. ${ }^{2}$; Scheideler, M. ${ }^{3}$; Trajanoski, Z. ${ }^{3}$; \\ Egger, G. ${ }^{2}$; Kenner, L. ${ }^{2}$; Greil, R. ${ }^{1}$; Merkel, O. ${ }^{1}$ \\ 13. Med. Abteilung, Landeskrankenhaus Salzburg, Labor für \\ Immunologische und Molekulare Krebsforschung, Salzburg, Austria, \\ ${ }^{2}$ Ludwig Boltzmann Institut, Wien, Austria, ${ }^{3}$ TU Graz, Graz, Austria
}

Introduction: Anaplastic large cell lymphoma (ALCL) is an aggressive nonHodgkin T-cell lymphoma. About half of the ALCL-patients bear a chromosomal translocation linking the nucleophosmin (NPM) gene to the anaplastic lymphoma kinase (ALK) resulting in constitutive activation of ALK. This causes the induction of oncogenic downstream pathways including PI3K/Akt/ mTOR, cJun/JunB, c-myc and Jak/STAT. Mainly due to their different prognosis the WHO has classified $\mathrm{ALK}^{+}$and $\mathrm{ALK}^{-}$ALCL as two different disease entities. In this study we have defined a specific miRNA signature of ALCL using extensive miRNA profiling in different ALCL model systems as well as in primary tissues.

Methods: Cell lines were NPM-ALK positive Karpas 299, SR786, SU-DHL1 and NPM-ALK negative Mac2a and FE-PD. microRNA arrays contained 2056 capture probes using linked nucleic acid technology. Cells were transfected with pre-miRNA oligonucleotides using lipofection. Q-PCR was performed using Taq-Man probes. Balb/c immunodeficient (SCID) mice were employed for xenograft experiments.

Results: 6 miRNAs that were specifically over-expressed in $\mathrm{ALK}^{+}$cell lines could discriminate $\mathrm{ALK}^{+}$from $\mathrm{ALK}^{-}$samples in primary ALCL tissue. Five of these belonged to the oncogenic miR-17 92 cluster and had identical seed regions (miR-17, miR-20a, miR-20b, miR-93 and miR-106) suggesting common target proteins. The last of the 6 candidates, miR-886-3p, was found to be located near the translocation point of NPM-ALK. Furthermore, we identified a set of 4 miRNAs that were concomitantly down-regulated in ALCL cell lines, the murine NPM-ALK mouse model as well as in primary ALCL tissue specimens (miR-29a, miR-29c, miR-101 and miR-150). Overexpression of miR-101 resulted in reduced proliferation of ALK ${ }^{+}$Karpas 299 and SR786 cell lines. Validated targets of miR-101 include the antiapoptotic Mcl-1, the histone mehtyltransferase EZH2 and the mTOR protein. Inhibition of the miR101 target mTOR by temsirolimus resulted in severely reduced tumour growth in Balb/c mice engrafted with murine ALK+ ALCL cell lines.

Conclusions: We have defined miRNAs that are over-expressed in ALK ALCL and characterized a set of miRNAs deregulated in ALCL irrespective of ALK status. Over-expression of miR-101 reduced ALCL cell line proliferation and inhibition of its target mTOR led to reduced tumour growth in ALCL mouse models. miR-101 in addition to other members of the miRNA signature may offer novel therapeutic avenues for ALCL.

Disclosure: No conflict of interest disclosed.

\section{Freie Vorträge \\ Stammzelltransplantation 3}

V700

Cytokeratin-18 is a useful serum biomarker for the early detection of acute intestinal graft-versus-host disease

Waterhouse, M. ${ }^{1}$; Samek, E. ${ }^{1}$; Bertz, H. ${ }^{1}$; Finke, J.

${ }^{1}$ Universitätsklinikum Freiburg, Hematologie/Onkologie, Freiburg, Germany

Cytokeratin-18 (CK18) is an intermediate filament in epithelial and parenchymal cells. Is expressed in colon, placenta, liver and lymph nodes of breast carcinoma. This protein is cleaved by caspases during epithelial cell apoptosis and fragments are released into the serum. Since apoptosis is one of the hallmarks of graft-versus-host disease (GvHD), CK18 can potentially be used as a biomarker for tissue damage during apoptosis. GvHD is a frequent and life threatening complication after allogeneic hematopoietic cell transplantation (HCT). Histological diagnosis of acute GvHD is not always feasible, since the affected tissues are not reachable due to different clinical conditions. Therefore a serum biomarker in this setting is a valuable resource for GvHD presumptive diagnosis. We analyze CK18 serum levels in patients undergoing allogeneic HCT and correlate these levels with clinical complications after transplant. Serum samples were obtained from 38 patients before and after transplant at different time points. Patient serum levels pre-transplant showed no statistical significant difference with serum obtained from healthy individuals. In $20 \%$ of patients without acute GvHD increments in serum CK18 were detected, while almost all patients (92\%) with acute intestinal GvHD showed increments of more than $50 \%$ respect with the pre-transplant sample. Sensitivity of CK18 for intestinal GvHD detection was 0.92 , while the negative predictive value was 0.91 . For skin GvHD sensitivity was 0.60 with a negative predictive value of 0.71. Significant increments of CK18 serum levels after transplant, were associated with development of chronic GvHD. Interestingly CK18 levels were raised before clinical GvHD manifestations in 10 out of 12 patients with acute intestinal GvHD (83\%), while the remaining two patients had late onset intestinal GvHD (day $>100)$. This pattern was not observed in 10 patients with only acute skin GvHD. Our findings suggest that serum CK18 is a predictive and sensitive biomarker for acute intestinal GvHD. It can be used for screening and as a surrogate marker for patients undergoing allogeneic HCT in whom a gut biopsy is clinically indicated but not feasible to perform.

Disclosure: No conflict of interest disclosed.

\section{V701}

\section{GPI-anchor negative T cells with impaired effector function persist after Alemtuzumab mediated T-cell depletion}

\section{Lay, A.N. ${ }^{1}$; Goetze, C. ${ }^{1}$; Wolff, D. ${ }^{1}$; Theobald, M. ${ }^{1}$; Herr, W. ${ }^{1}$;} Meyer, R.G. ${ }^{1}$; Wagner, E.M. ${ }^{1}$

${ }^{1}$ University Medical Center Mainz, Hematology/Oncology, Mainz, Germany

CD52 is a glycosylphosphatidylinositol (GPI)-anchored surface antigen with unknown function. It is targeted by Alemtuzumab, a monoclonal antibody frequently used for T-cell depletion (TCD) in the context of allogeneic hematopoietic stem cell transplantation (HSCT). We previously demonstrated that significant proportions of reconstituting $\mathrm{T}$ cells remained $\mathrm{CD} 52^{\text {neg }}$ for years after Alemtuzumab-mediated TCD in the context of allogeneic HSCT. We analyzed $\mathrm{T}$ cells of 20 patients following this protocol by co-labeling CD52 with FLAER, a fluorescent aerolysin particularly binding to GPIanchors. Thereby we demonstrated that the loss of CD52-expression was associated with the loss of GPI-anchors themselves. As many surface proteins are attached to the cell-surface by GPI-anchors, we wondered whether this had an impact on T-cell function. We therefore separated CD52 ${ }^{\text {neg }}$ from CD52 $2^{\text {pos }}$ CD4 and CD8 $\mathrm{T}$ cell populations by flow cytometry, expanded them in vitro (IL2, Okt3, feeder-cells) and observed constant absence of GPI-anchors for over 16 weeks in culture. The growth of $\mathrm{CD} 52^{\text {neg }}$ did not differ from that of CD52 ${ }^{\text {pos }} \mathrm{T}$ cell cultures. However, after stimulation with CMV-pp65 peptides, proliferation of CD52 $2^{\text {pos }} \mathrm{CD} 4$ and CD8 T-cells was enhanced compared to that of CD52 ${ }^{\text {neg }} \mathrm{T}$ cells. We also tested $\mathrm{T}$ cells from these cultures with CMVpeptide loaded dendritic cells (DC) in IFN-g ELISPOT assays and detected specific spot production among the GPI-anchor positive T cells only. To investigate the function of T cells directly ex vivo, we performed IFN-g Secretionassays and used autologous DC pulsed with CMV-peptides or allogeneic DC as stimulators. Again, IFN-g secretion was reduced in CD52 $2^{\text {neg }}$ compared to CD52 ${ }^{\text {pos }} \mathrm{T}$ cells. Despite the functional differences, the sub-populations did not differ regarding the expression of naïve and memory markers. In addition, T cells with CMV-pp65 specific T-cell receptors were present in the CD52 $2^{\text {pos }}$ and the CD52 $2^{\text {neg }}$ subpopulation as confirmed by HLA-tetramer staining. In summary, significant proportions of reconstituting $\mathrm{T}$ cells in our patients lost the expression of GPI-anchors after Alemtuzumab-based TCD. GPIanchor negative $\mathrm{T}$ cells exhibited a reduced proliferative capacity and a reduced IFN-g secretion in response to CMV-peptides and allogeneic DC. Our data suggests that a loss of GPI-anchored molecules might contribute to an impaired function of reconstituting donor $\mathrm{T}$ cells after Alemtuzumab-based TCD.

Disclosure: No conflict of interest disclosed. 
V702

\section{Transition from CD103+ to CD11b+ dendritic cells correlates with acute intestinal graft-versus-host disease}

Jordán Garrote, A.-L. ${ }^{1,2}$; Brede, C. ${ }^{3}$; Bäuerlein, C.A. ${ }^{3} ;$ Riedel, S.S. ${ }^{3}$, Kiesel, C. ${ }^{3}$; Grether, M. ${ }^{4}$; Mattenheimer, K. ${ }^{3}$; Ritz, M. ${ }^{3}$; Baker, J. ${ }^{5}$; Negrin, R.S. ; Einsele, H. ${ }^{3}$; Schulz, S. ${ }^{4}$; Beilhack, A. ${ }^{3}$

${ }^{1}$ Universitätsklinikum Würzburg, Medizinische Klinik und Poliklinik II, Würzburg, Germany, ${ }^{2}$ Graduate School of Life Science, Würzburg, Germany, ${ }^{3}$ Universitätsklinikum Würzburg, Würzburg, Germany, ${ }^{4}$ Technische Universität München, München, Germany, ${ }^{5}$ Stanford University, Stanford, United States

Recipient dendritic cell - donor $\mathrm{T}$ cell interactions in secondary lymphoid organs are essential to initiate acute graft-versus-host disease (aGVHD). Nevertheless, it remains unclear whether peripheral mucosal DCs are required to maintain the immune response in advanced aGVHD.

We aimed to investigate whether DCs in the intestinal mucosa are required to perpetuate aGVHD. To address this question we used a murine allogeneic hematopoietic cell transplantation (allo-HCT) model [luciferase ${ }^{+}$FVB/N $\left(\mathrm{H}-2^{\mathrm{q}}\right)$ or $\mathrm{C} 57 \mathrm{Bl} / 6\left(\mathrm{H}-2^{\mathrm{b}}\right)$ splenocytes (CD90.1)] plus wild type bone marrow into myeloablative conditioned allogeneic Balb/c recipients (H-2 ${ }^{\mathrm{d}}, \mathrm{CD} 90.2$ $8 \mathrm{~Gy})$.To visualize cell migration we employed non-invasive bioluminescence imaging. Phenotyping studies were performed by using multiparameter flow cytometry, and cell interactions were analyzed by fluorescence/confocal microscopy.

Histological analysis of the intestinal mucosa revealed high numbers of DC donor $\mathrm{T}$ cell contacts during advanced aGVHD. Persisting host APCs (I-A $\left.{ }^{\mathrm{d}}\right)$ co-localized with both, $\mathrm{CD} 4^{+} \mathrm{CD} 90.1^{+}$and $\mathrm{CD} 8^{+} \mathrm{CD} 90.1^{+}$donor $\mathrm{T}$ cell subtypes. Confocal microscopy confirmed interactions between DCs and donor T cells and revealed engagement of costimulatory molecules, such as CD80, at sites of contact. Although myeloablative conditioning augmented the numbers of infiltrating alloreactive $\mathrm{T}$ cell in the intestinal tract, we observed no reduction in DC - donor T cell contacts in non-conditioned recipients when compared to conditioned Balb/c Rag ${ }^{-1 /}$ common-g-chain ${ }^{-/}$allo-HCT recipients Next we investigated which peripheral mucosal DC subtypes engaged with donor $\mathrm{T}$ cells. DC subsets shifted from migratory $\mathrm{CD} 103^{+} \mathrm{CD} 11 \mathrm{~b}^{\mathrm{lo}} \mathrm{CD} 11 \mathrm{c}^{+}$ DCs, observed during steady state conditions, to a dominating CD103 $\mathrm{CD} 11 \mathrm{~b}^{\text {hi }} \mathrm{CD} 11 \mathrm{c}^{+} \mathrm{DC}$ population during the aGVHD effector phase. Strikingly, the number of host mucosal DCs did not decrease 6 days later after donor cell transfer, and were negative for the proliferation marker Ki67. Mucosal DC were negative for the proliferation marker Ki67, suggesting a continuous refill of this compartment with monocyte derived DCs arising from circulating monocytes attracted to areas of inflammation.

Our data provide evidence for a likely underappreciated role of mucosal DCs during later stages of aGVHD. This suggests that disruption of mucosal DC donor $\mathrm{T}$ cell interactions may provide a promising target for therapeutic approaches in patients with ongoing GVHD.

*Equal contribution of S.S. and A.B

Disclosure: No conflict of interest disclosed.

\section{V703}

\section{Progression-free survival of recipients with HLA-C KIR ligand group 2 homozygosity is impaired following peripheral blood stem cell transplantation (PBSCT) but not after bone marrow transplantation (BMT)}

\section{Clausen, J. ${ }^{1}$; Kircher, B. ${ }^{1}$; Gastl, G. ${ }^{1}$; Nachbaur, D. ${ }^{1}$}

${ }^{1}$ Medizinische Universität Innsbruck, Innere Medizin V, Innsbruck, Austria

Introduction: We have recently reported the superior progression-free survival of recipients with HLA-C KIR ligand heterozygosity (C1/C2), particularly as opposed to HLA-C group 2 homozygosity (C2/C2), in matched related, T cell replete PBSCT (Clausen et al; BBMT; 2010). Here, we test the hypothesis that the deleterious effect of $\mathrm{C} 2$ group homozygosity may be reversed in BMT and in T cell-depleted transplantation.

Methods: In a retrospective analysis of a single centre cohort of stem cell transplants for adults with various haemato-oncological diseases, 75 out of 317 transplants were performed as BMT, while 242 were PBSCT. 146 trans- plants $(46 \%)$ were unrelated, and 60 (19\%) were mismatched at 1-2 loci. Haploidentical SCT were not included. Median follow-up of survivors was 39 months (range, 3.1 - 253). In 94 transplants, in-vivo T cell depletion was applied, mainly by using anti thymocyte globulin.

Results: 120 recipients $(38 \%)$ were $\mathrm{C} 1 / \mathrm{C} 1$ and $50(16 \%)$ were $\mathrm{C} 2 / \mathrm{C} 2$ homozygous, while 147 (46\%) were C1/C2 heterozygous. Following PBSCT, the 3-year Kaplan-Meier estimate for progression-free survival (PFS) was 0.23 for $\mathrm{C} 2 / \mathrm{C} 2$ homozygous recipients, compared to 0.36 and 0.41 for $\mathrm{C} 1 / \mathrm{C} 1$ and $\mathrm{C} 1 / \mathrm{C} 2$ recipients $(\mathrm{p}=0.07)$. Following sibling donor PBSCT $(\mathrm{n}=130)$, the respective probabilities were $0.14(\mathrm{C} 2 / \mathrm{C} 2), 0.36(\mathrm{C} 1 / \mathrm{C} 1)$, and $0.52(\mathrm{C} 1 / \mathrm{C} 2$; $\mathrm{p}=0.002$ ). Multivariate analysis of PBSCT confirmed the adverse effect of $\mathrm{C} 2 /$ C2 homozygosity on PFS (RR 1.62; $\mathrm{p}=0.03$ in PBSCT, and RR 2.31; $\mathrm{p}=0.007$ in sibling donor PBSCT). In contrast, following BMT, 3-y PFS of C2/C2 recipients $(0.65)$ was at least comparable to that of $\mathrm{C} 1 / \mathrm{C} 1(0.35)$ and $\mathrm{C} 1 / \mathrm{C} 2$ recipients $(0.26 ; \mathrm{p}=0.26)$. Vice versa, the graft source had no significant impact on PFS in $\mathrm{C} 1 / \mathrm{C} 1$ and $\mathrm{C} 1 / \mathrm{C} 2$ recipients. However, $\mathrm{C} 2 / \mathrm{C} 2$ recipients had superior 3y-PFS and overall survival in case of BMT $(0.59$ and 0.65$)$ compared to PBSCT $(0.23$ and $0.20 ; \mathrm{p}=0.01$ and $\mathrm{p}=0.023$, respectively). The HLA-C group had no significantly different impact on PFS in the TCD vs the non-TCD cohort.

Conclusion: According to the present data, the graft source is the major factor influencing effects of HLA-C KIR ligands. Particularly, C2/C2 recipients may fare better with a BM than with a PBSC graft. Our data do not support the decision for or against TCD on the basis of HLA-C constellations.

Disclosure: No conflict of interest disclosed

\section{V704}

\section{Diagnostic evaluation of EBV-associated} lymphoproliferative disorders after allogeneic stem cell transplantation: A single centre retrospective analysis.

\section{Baumann, P. ${ }^{1}$; Groß-Judith, $P .{ }^{1}$; Judith, $D .^{2}$; Schleuning, M. ${ }^{2}$;} Girgensohn, S. ${ }^{3}$; Schwerdtfeger, $R^{2}$; Baurmann, $H^{2}$

${ }^{1}$ Bioscientia Institut für Medizinische Diagnostik $\mathrm{GmbH}$, Labor an der Deutschen Klinik für Diagnostik, Wiesbaden, Germany, ${ }^{2}$ Deutsche Klinik für Diagnostik, BMT-Unit, Wiesbaden, Germany, ${ }^{3}$ Bioscientia Institut für Medizinische Diagnostik GmbH, Labor Ingelheim, Ingelheim, Germany

Introduction: Epstein Barr Virus (EBV)-associated post-transplant lymphoproliferative disorder (PTLD) is a serious and underestimated complication of allogeneic stem cell transplantation (alloSCT). Valuable diagnostic tools are needed to help establish the diagnosis early.

Patients and methods: We retrospectively analyzed 24 cases of EBVassociated PTLD diagnosed in our centre among 887 consecutive patients (pts) following related $(\mathrm{n}=300)$ or unrelated $(\mathrm{n}=587)$ allogeneic transplantation between 1997 and 2009. Special attention was paid to cytological and immunophaenotypical changes of peripheral blood (PB) cells at the time of diagnosis.

Results: 10 female and 14 male pts with probable or definite EBV-PTLD were selected on the basis of their records following alloSCT. Median (md) age was 55 (22-62) years at transplant. 9 pts had received their graft from a well matched, 6 from a partially matched, 6 from a mismatched unrelated donor, and three from a related donor. Most pts had positive EBV-serology whereas 3 pts were serologically negative before alloSCT and acquired primary infection. All but one pt had been treated with ATG. Md time of diagnosis was day (d) +58 (28-378), with one exceptional case before d0. In 17 pts the diagnosis was proven by histology ( 7 monomorphic, 9 polymorphic and one unclassified lesions), whereas in the remaining cases positive EBV-PCR from PB and typical clinical symptoms established the diagnosis leading to an overall incidence of 2,7\% PLTD. From 14 pts PB cytology and immunophaenotyping (IP) were available at the time of diagnosis: In 5 pts IP was diagnostic and in another 5 pts suspicious for the diagnosis. Typical cytological findings ranged from a mononucleosis-like picture with few immunoblasts and plasma cells to various numbers of circulating lymphoma cells. IP revealed a spectrum from CD8-cell expansion with low numbers of polyclonal, activated B-cells to predominantly oligoclonal or monoclonal B-cell proliferations with a typical CD19+, CD20+, CD38++ antigen pattern. 16 pts received Rituximab®, combined with either DLI, radiation or chemotherapy in 5 pts. One CD20-negative pt was treated with chemotherapy and radiation alone. 5 pts are alive, at a md 
follow up of 864 (505-3390) ds. The remaining pts died at a md of 99 ds, 14 from direct consequences of PTLD.

Conclusion: PTLD following alloSCT carries a dismal prognosis. Cytology and IP of PB together with quantitative EBV-PCR are helpful in establishing the diagnosis.

Disclosure: No conflict of interest disclosed.

V705

Efficacy of allogeneic-SCT in patients with AML and FLT-3 mutations

Schleuning, M. ${ }^{1}$; Judith, D. ${ }^{1}$; Heshmat, M. ${ }^{1}$; Burlakova, I. ${ }^{1}$; Müller, J. ${ }^{1}$; Jedlickova, Z. ${ }^{1}$; Salm, E. ${ }^{1}$; Baurmann, $H_{.}{ }^{1}$; Schwerdtfeger, R. ${ }^{1}$

${ }^{1}$ Deutsche Klinik für Diagnostik, Zentrum für Blutstammzell- und Knochenmarktransplantation, Wiesbaden, Germany

Patients with acute myeloid leukemia (AML) with FLT-3 mutations have an extremely high risk of relapse after conventional chemotherapy. The role of allogeneic stem cell transplantation (SCT) for this patient cohort has been discussed controversially in recent years. This report summarizes our cumulative experience in a cohort of 42 consecutive patients (age 17-70, median 5 years) allografted for FLT-3 positive AML in a single centre. In more than $80 \%$ a FLAMSA-RIC based conditioning regimen was used, in 5\% BCNU/ Melphalan/Fludarabine, and in $14 \%$ conventional radiation- or Busulfanbased regimens. Most patients received mobilized peripheral blood stem cells as graft and 10 patients had a sibling and 32 an unrelated donor (MUD), respectively. Half of the patients were allografted in complete remission and twenty with active, mostly refractory disease. With a median follow-up for surviving patients of nearly 2 years (range 64 - 1746 days) the Kaplan-Meyer procedure estimates a $48 \%$ probability of survival at 2 y after transplantation. Interestingly, there is no difference what so ever in survival if patients had an identical sibling donor or a MUD. Similarly, neither patient age below or above the median, nor the applied conditioning regimen did affect the outcome. The only significant variable for improved survival was being in complete remission at transplantation with a 2-year overall survival probability of $60 \%$ as compared to $30 \%$ for patients with active disease. Thirteen patients (31\%) relapsed after allografting, which is substantially lower as to what is reported after conventional chemotherapy. Three of these patients could be salvaged by a second transplant, whereas 10 patients finally died from leukaemia. Non relapse mortality was $24 \%$ with 2 patients dying from acute GVHD, 7 from infections and 1 from suicide, despite being well physically. In conclusion, our data support the notion that allogeneic SCT is a highly effective treatment option for patients with AML and FLT-3 mutations and that, if the patient is eligible, it should be undertaken whenever possible in 1. complete remission. However, even patients with primary induction failure have a reasonable chance to be salvaged by allogeneic SCT.

Disclosure: No conflict of interest disclosed.

\section{Freie Vorträge \\ ALL}

V706

Methylation analysis and gene expression profiling of IGFBP7 reveal insights into its role in acute leukemia

Heesch, S. ; Bartram, I. ${ }^{1}$; Neumann, M. ${ }^{1}$; Reins, J. ${ }^{1}$; Mossner, M. ${ }^{1}$; Schlee, C. ${ }^{1}$; Stroux, A. ${ }^{2}$; Haferlach, T. ${ }^{3}$; Hofmann, W.-K. ${ }^{4}$ Thiel, E. ${ }^{1}$; Baldus, C.D. ${ }^{1}$

${ }^{1}$ Charité Universitätsmedizin Berlin, Campus Benjamin Franklin, Medizinische Klinik III Hämatologie, Onkologie und Transfusionsmedizin Berlin, Germany, ${ }^{2}$ Charité Universitätsmedizin Berlin, Campus Benjamin Franklin, Institut für Biometrie und klinische Epidemiologie, Berlin, Germany, ${ }^{3}$ MLL Münchner Leukämie Labor, München, Germany, ${ }^{4}$ Universität Heidelberg, Medizinische Fakultät Mannheim, Medizinische Klinik III, Mannheim, Germany

Background and aims: The gene IGFBP7 (Insulin-like Growth Factor Binding Protein 7) was recently identified as a novel molecular marker in acute leukemia. IGFBP7 is aberrantly expressed in subsets of acute myeloid leukemia (AML) and lymphoblastic leukemia (ALL) with high mRNA expression levels predicting primary therapy resistance in T-ALL patients. However, the regulation of aberrant $I G F B P 7$ expression and its potential role in acute leukemia remain unknown.

Methods: In order to investigate mechanisms underlying aberrant IGFBP7 expression, we used pyrosequencing technology to study the DNA-methylation of IGFBP7 in 149 acute leukemia patient samples and healthy donors. In addition, the human T-cell leukemia cell line BE-13 was treated with the demethylating agent decitabine to validate IGFBP7 regulation by DNA-methylation. Quantitative RT-PCR was performed to determine the relative expression levels of $I G F B P 7$ in all samples. Furthermore, we analyzed gene expression profiles (GEP) of 86 T-ALL patients, generated from the MILE Stage I study (HG-U133 Plus 2.0, Affymetrix) to unravel IGFBP7 associated gene expression pathways. Patient samples were divided into a low $(\mathrm{n}=57)$ and high $(\mathrm{n}=29)$ expression group according to $I G F B P 7$ microarray expression data. Differentially expressed genes between low and high IGFBP7 groups were defined with a minimum expression change of threefold $(P<0.005)$.

Results: Methylation analyses revealed aberrant methylation of IGFBP7 in T-ALL $(\mathrm{n}=109)$ and AML $(\mathrm{n}=40)$ patients. Moreover, the mRNA expression level of $I G F B P 7$ was negatively correlated with the methylation status in acute leukemia. This finding was further supported by the in vitro study, which showed restored IGFBP7 expression and a decreased methylation after decitabine treatment of BE-13 cells. Furthermore, in T-ALL hypomethylation was associated with immature features such as an early immunophenotype and expression of the hematopoietic stem cell markers CD117 $(P<0.001)$ and CD34 $(P<0.001)$. In accordance, GEP of T-ALL patients revealed up-regulation of stem cell markers (CD34 and $C D 133)$ and genes associated with poor outcome and pathogenesis of leukemia $(M N 1, B A A L C, F L T 3)$ in the high IGFBP7 expression group.

Conclusion: Our study demonstrated that aberrant IGFBP7 expression in acute leukemia is regulated by DNA-methylation. Over-expression of the $I G F B P 7$ gene is likely to characterize an immature and a more malignant subtype of acute leukemia.

Disclosure: No conflict of interest disclosed.

\section{V707}

\section{Bcr-Abl-mediated oncogenic stress induces ER stress response and a programmed necrosis-like cell death in Bcr-Abl overexpressing Imatinib-resistant ALL cells}

\author{
Dengler, M.A. ${ }^{1}$; Staiger, A. ${ }^{1}$; Gutekunst, M. ${ }^{1}$; van der Kuip, H. ${ }^{1}$; \\ Aulitzky, W.E. ${ }^{2}$
}

${ }^{1}$ Dr. Margarete Fischer-Bosch-Institute of Clinical Pharmacology and University of Tübingen, Stuttgart, Germany, ${ }^{2}$ Robert Bosch Hospital, Hämatologie, Onkologie und Palliativmedizin, Stuttgart, Germany

Imatinib resistance is a major problem in treatment of Bcr-Abl positive leukemias, particularly in patients with ALL and advanced CML. One mechanism of this resistance is overexpression of Bcr-Abl.

We investigated the effects of Imatinib deprivation in Bcr-Abl overexpressing Imatinib-resistant ALL cell lines. Removal of Imatinib from culture medium led to a delayed induction of cell death starting approximately 40 hours after Imatinib deprivation. This cell death was preceded by a robust induction of Bcr-Abl autophosphorylation and concomitant overstimulation of PI3K-, MAPK-, and JAK/STAT signalling. Imatinib deprivation also caused an elevated intracellular ATP and protein content accompanied by a massive increase in cell size and cytoplasmic vacuolization. To determine if Imatinib deprivation induced an ER stress response concurrent with vacuolization we investigated CHOP expression and eIF2alpha phosphorylation. Indeed, upon removal of Imatinib a massive induction of CHOP expression and eIF2 alpha phosphorylation could be detected. Severe ER stress induces cell death either by a BIM dependent apoptosis or in a TRAF-RIP1 dependent way. Besides the observation of a robust induction of BIM, Imatinib deprivation induced cell death was completely independent of Caspase activation. We hypothesize that BIM may be antagonized by Bcl-xL which was massively induced in parallel to BIM upon removal of Imatinib. Interestingly, the RIP1 inhibitor Necrostatin partially rescued the cells upon Imatinib deprivation. Using a protein kinase inhibitor library, we also identified inhibitors of glycogen synthase kinase-3 (GSK3) and p38-MAPK as potent compounds which prevented induction of cell death upon removal of Imatinib. A posttranslational modification of RIP 
which was seen selectively in cells after Imatinib deprivation was also completely absent in cells pre-treated with these inhibitors indicating a central role of RIP1 for oncogenic stress-induced cell death.

In conclusion, upon Imatinib deprivation we found a massive induction of a necrosis-like cell death in Bcr-Abl overexpressing, Imatinib resistant ALL cells as a consequence of Bcr-Abl over-activation induced ER stress. These data implicate that a discontinuous treatment with Imatinib (periods of Imatinib treatment followed by short periods without Imatinib) may prevent the appearance of cell clones with Bcr-Abl overexpression.

Disclosure: No conflict of interest disclosed.

\section{V708}

IAP inhibitors render childhood leukemia cells sensitive

for TRAIL- or chemotherapy-triggered apoptosis, overcome resistance by Bcl-2 and exert anti-leukemic activity in a NOD/SCID mouse model in vivo

Fakler, M. ${ }^{1}$; Löder, S. ${ }^{1}$; Jeremias, I. ${ }^{2}$ Debatin, K.-M. ${ }^{1}$; Fulda, S. ${ }^{1}$ ${ }^{1}$ Klinik für Kinder- und Jugendmedizin, Universität UIm, UIm, Germany, ${ }^{2}$ Helmholtz Center, München, Germany

Introduction: Defects in apoptosis contribute to poor outcome in high risk pediatric acute lymphoblastic leukemia (ALL), calling for novel strategies that counter apoptosis resistance. "Inhibitor of Apoptosis" (IAP) proteins present promising targets, since they are expressed at high levels in acute leukemia. Methods: We investigated the effect of small molecule IAP inhibitors alone and in combination with TRAIL or chemotherapeutics in ALL cell lines, primary leukemic blasts, normal peripheral blood lymphocytes and in a mouse model of pediatric ALL.

Results: IAP inhibitors at subtoxic concentrations, but not a structurally related control compound, cooperate with TRAIL to induce apoptosis in ALL cells in a highly synergistic manner as calculated by combination index. Also, IAP inhibitors act in concert with TRAIL to reduce clonogenic growth of ALL cells demonstrating that they suppress longterm survival. Analysis of signaling pathways reveals that IAP inhibitors enhance TRAIL-induced activation of caspases, loss of mitochondrial membrane potential and cytochrome c release in a caspase-dependent manner. Intriguingly, IAP inhibitors overcome Bcl-2mediated resistance to TRAIL by promoting Bcl-2 cleavage, Bak conformational change and caspase-3-driven mitochondrial perturbations. Moreover, IAP inhibitors sensitize ALL cells for chemotherapy, e.g. cytarabine, doxorubicin, etoposide and 6-mercaptopurine. This chemosensitization is inhibited by a TNFalpha-neutralizing antibody, demonstrating that it depends on IAP inhibitor-triggered production of TNFalpha. Of note, IAP inhibitors potently trigger cell death in leukemic blasts from children with ALL ex vivo and also sensitize primary leukemia cells for TRAIL- or chemotherapy-induced cell death. In contrast to malignant cells, IAP inhibitors and TRAIL at equimolar concentrations are non-toxic to normal peripheral blood lymphocytes despite expression of the apoptosis-inducing TRAIL receptors on the cell surface, pointing to a therapeutic window. Most importantly, IAP inhibitors exert potent anti-leukemic activity in vivo in a mouse model of pediatric ALL engrafted in NOD/SCID mice.

Conclusions: These findings provide first evidence that IAP inhibitors present a novel strategy to prime childhoodacute leukemia cells for TRAIL- or chemotherapy-induced cell death. These data have important clinical implications for the development of apoptosis targeted therapies for childhood leukemia.

Disclosure: No conflict of interest disclosed.
V709

Long term follow up of elderly patients with PhiladelphiaPositive Acute Lymphoblastic Leukaemia (Ph+ALL): Updated results of the GMALL elderly trials

Pfeifer, H. ${ }^{1}$; Wystub, S. ${ }^{1}$; Voelp, C. ${ }^{1}$; Wassmann, B. ${ }^{1}$; Brueck, P. ${ }^{1}$; Giagounidis, A. ${ }^{2}$; Stelljes, M. ${ }^{3}$; Dührsen, U. ${ }^{4}$; Schmalzing, M.5. Schaich, M. ${ }^{6}$; de Wit, M. ${ }^{7}$; Ganser, A. ${ }^{8}$; Fritsch, G. ${ }^{9}$; Gassmann, W. ${ }^{10}$; Pfreundschuh, M. ${ }^{11}$; Müller, M. ${ }^{12}$; Wendelin, K. ${ }^{13}$; Schmid, M. ${ }^{14}$; Beck, H.-J. ${ }^{15}$; Dengler, J. ${ }^{16}$; Horst, H.A. ${ }^{17}$; Lübbert, M. ${ }^{18}$; Serve, H. ${ }^{1}$; Hoelzer, D. ${ }^{1}$; Ottmann, O. ${ }^{1}$

${ }^{1}$ Goethe-Universität, Medizinische Klinik II, Hämatologie/Onkologie, Frankfurt, Germany, ${ }^{2}$ St. Johannes Hospital, Medizinische Klinik II, Duisburg, Germany, ${ }^{3}$ Universitätsklinikum Münster, Münster, Germany, ${ }^{4}$ Zentrum für Innere Medizin, ME der Universitäts-GHS Essen, Essen, Germany, ${ }^{5}$ Universitätsklinik, Tübingen, Germany, ${ }^{6}$ Universitätsklinikum Carl Gustav Carus, Dresden, Germany, ${ }^{7}$ Klinikum Neukölln, Berlin, Germany, ${ }^{8}$ Medizinische Hochschule Hannover, Hannover, Germany, ${ }^{9}$ Städtisches Klinikum Braunschweig, Braunschweig, Germany, ${ }^{10} \mathrm{St}$. Marien-Krankenhaus, Siegen, Germany, ${ }^{11}$ Universitätskliniken Homburg/ Saar, Homburg/Saar, Germany, ${ }^{12}$ Klinikum Mannheim, Mannheim, Germany, ${ }^{13}$ Klinikum Nord der Stadt Nürnberg, Nürnberg, Germany, ${ }^{14}$ Universitätsklinik UIm, Ulm, Germany, ${ }^{15}$ Uniklinik Mainz, Mainz, Germany, ${ }^{16}$ Universitätsklinikum Heidelberg, Heidelberg, Germany, ${ }^{17}$ Universitätsklinikum Schleswig Holstein Campus Kiel, Kiel, Germany, ${ }^{18}$ Albert Ludwigs-University Medical Centre Freiburg, Freiburg, Germany

Background: Introduction of Imatinib in the treatment of patients with $\mathrm{Ph}+\mathrm{ALL}$ have resulted in a high complete remission exceeding $90 \%$, but the long-term response duration is still unsatisfactory. It has not been established whether results of combined TKI with induction and consolidation therapy are superior to TKI monotherapy. Here, we provide the extended and updated results of the GMALL studies in patients older than 55 years, who were not initially considered for allo SCT.

Study design and patients demographics: The design of the first GMALL elderly study of TKI and chemotherapy has been described previously and consisted of a randomized induction comparing imatinib (IM) and chemotherapy (CTX), followed by up to 6 cycles of consolidation chemotherapy combined with imatinib (Ottmann, Cancer 2007). In a subsequent single arm study patients received imatinib induction followed by the same combination of imatinib and consolidation chemotherapy. 111 patients (109 ALL pts., 2 CML, lyBC pts.; median age 66 years, range 54- 80 yrs; male/female $n=51 / 60$;) were enrolled. 66 pts. received an imatinib induction, 37 pts. an induction with chemotherapy and 8 pts. received chemotherapy induction in conjunction with imatinib.

Results: The overall CR rate was $86 \%$ (pts. with IM induction: $89 \%$, pts. with CTX induction: $84 \%$, pts. with combination treatment: $84 \%$ ). 3 patients were primary refractory, 2 on imatinib, 6 patients died during induction. The median OS of all patients was 14.7 months (range $15 \mathrm{~d}-81.4$ mo.) There was no significant different OS between the three induction types: 18 months IM-induction versus 15 mo. CTX-induction versus 9 mo. combined induction. The remission duration was independent of the initial induction type, too (381 d IM-Ind. vs. 400 d CTX-Ind. vs. 159 d Comb-Ind.).

11 pts. (median age $61 \mathrm{y}$, range 54-66) were transplanted in CR 1 . The time from diagnosis to SCT was 141 days ( $94 \mathrm{~d}-509 \mathrm{~d}$ ). With a median follow-up of 19.3 mo (range $4.8 \mathrm{mo}-54.5 \mathrm{mo}$ ), 6 patients are in ongoing CR with a median OS of 41.5 mo. (range 9.5 mo - $54.5 \mathrm{mo}$ ), 2 pts. died in CR, 3 pts. relapsed.

Conclusions: The combination of imatinib in conjunction with intensive chemotherapy is feasible, but does not abrogate a high rate of relapse. As allogeneic SCT in first CR is able to sustain remissions in a substantial proportion of cases, transplantation should be strongly considered even in elderly $\mathrm{Ph}+\mathrm{ALL}$ pts.

Disclosure: Heike Pfeifer: No conflict of interest disclosed.

Oliver Ottmann: Honoraria: Novartis, BMS.; Financing of Scientific Research: Novartis, BMS. 
V710

\section{Definition of molecular remission in adult acute lymphoblastic leukemia (ALL) based on minimal residual disease (MRD)}

Gökbuget, N. ${ }^{1}$; Brüggemann, M. ${ }^{2}$; Arnold, R. ${ }^{3}$; Bartram, C.-R. ${ }^{4}$; Fietkau, R. ${ }^{5}$; Freund, M. ${ }^{6}$; Ganser, . $^{7}$; Kneba, M. ${ }^{2}$; Lipp, T. Ludwig, W.-D.9 ${ }^{9}$ Maschmeyer, G. ${ }^{10}$; Messerer, D. ${ }^{11}$; Raff, T. ${ }^{12}$; Rieder, H. ${ }^{13}$; Serve, H. ${ }^{1}$; Thiel, E. ${ }^{14}$; Hoelzer, D. ${ }^{1}$, German Multicenter Study Group for Adult ALL (GMALL)

${ }^{1}$ Klinikum der Goethe-Universität, Medizinische Klinik II, Frankfurt, Germany, ${ }^{2}$ Universitätsklinik Schleswig Holstein, Campus Kiel, Innere Medizin II, Kiel, Germany, ${ }^{3}$ Charité - Universitätsmedizin, Campus Virchow, Abteilung für Hämatologie und Onkologie, Berlin, Germany, ${ }^{4}$ RuprechtKarls-Universität, Institut für Genetik und Anthropologie, Heidelberg, Germany, ${ }^{5}$ Universitätsklinik, Strahlenklinik, Erlangen, Germany, ${ }^{6}$ Universitätsklinik, Abteilung für Hämatologie und Onkologie, Rostock, Germany, ${ }^{7}$ Medizinische Hochschule Hannover, Abteilung für Hämatologie, Hämostaseologie, Onkologie und Stammzelltransplantation, Hannnover, Germany, ${ }^{8}$ Krankenhaus Schwabing, Hematology/Oncology, München, Germany, ${ }^{9}$ Charite - Universitätsmedizin, Campus Buch, Hämatologie/ Onkologie, Berlin, Germany, ${ }^{10}$ Klinikum Ernst von Bergmann, Hämatologie/ Onkologie, Potsdam, Germany, ${ }^{11}$ Universität München, IBE, München, Germany, ${ }^{12}$ Universitätsklinik Schleswig Holstein, Campus Kiel, Hämatologie/Onkologie, Kiel, Germany, ${ }^{13}$ Universitätsklinik Düsseldorf, Zytogenetik, Düsseldorf, Germany, ${ }^{14}$ Charite - Universitätsmedizin Berlin Campus Benjamin Franklin, Abteilung für Hämatologie und Onkologie, Berlin, Germany

Achievement of CR confirmed by cytology is the most important prognostic factor in adult $\mathrm{AL}$ and hematologic $\mathrm{CR}$ and relapse are accepted study endpoints. MRD is a more sensitive, highly standardised method for response evaluation and an independent, strong prognostic factor. It is essential to define and validate MRD based response criteria - namely molecular-CR (molCR), molecular failure (molFAIL) and molecular relapse (molREL) - as new endpoints for clinical trials.

The German Multicenter Study Group for Adult ALL (GMALL) has conducted two consecutive studies (06/99 and 07/2003) with a risk and MRD adapted strategy. 1489 pts (15-55 y) with Ph-negative standard (SR) and high risk (HR) ALL have been included. MolCR was defined in pts with hematologic CR as negative MRD status after induction. MolREL was defined as reappearance of MRD above $10^{-4}$.

MolCR: The hematologic CR rate was $89 \%$ with differences between SR and HR ( $92 \%$ vs $84 \% ; \mathrm{p}<.0001)$ and age 35 yrs $(90 \%$ vs $86 \% ; \mathrm{p}=.01)$. The molCR was lower (69\%) in 479 evaluable pts. Differences were observed between SR vs HR $(77 \%$ vs $46 \% ; \mathrm{p}<.0001)$ and B vs T-lineage (63\% vs $79 \% ; \mathrm{p}=.0005)$. MolCR evaluation also allowed efficacy assessment of treatment modifications in induction.

SR pts were scheduled for chemotherapy. MolCR vs molFAIL pts had a superior overall survival (OS) $(67 \%$ vs $38 \%$; $<<.0001)$, mainly due to a lower relapse rate $(\mathrm{RR})(26 \%$ vs $59 \% ; \mathrm{p}<.0001)$. MolFAIL pts had a better OS with vs without $\mathrm{SCT}$ in CR1 (60\% vs $27 \% ; \mathrm{p}=.09)$.

HR pts were scheduled for SCT in CR1. OS was superior for MolCR vs MolFAIL (66\% vs $42 \%$; $\mathrm{p}=.003$ ). The RR was higher for molFAIL vs molCR on chemotherapy (66\% vs $18 \% ; \mathrm{p}=.04)$ and after SCT ( $34 \%$ vs $11 \% ; \mathrm{p}=.04)$. MolREL: 36 patients had an OS of 36\%.The probability of hematologic REL was $82 \%$ (median $75 \mathrm{~d}$ from molREL).

The data show the proof of principle for molecular response evaluation. MolFAIL is associated with significantly poorer outcome even after SCT. Pts with molFAIL and molREL are chemotherapy resistant and candidates for targeted, experimental drugs and SCT in CR1. Molecular response evaluation provides new refined endpoints for pivotal trials with new drugs, which can be measured early, by standardised methods and have strong correlation to clinical outcome.

Supported by Deutsche Krebshilfe 702657Ho2 and BMBF 01GI9971/8

Disclosure: No conflict of interest disclosed.
V711

\section{Allogeneic SCT in adult acute lymphocytic leukemia patients in CR1: Influence of patient age on outcome?}

Arnold, R. ${ }^{1}$; Terwey, T. ${ }^{1}$; Hemmati, P. ${ }^{1}$; Le Coutre, P. ${ }^{1}$; Vuong, L. ${ }^{1}$; Dörken, B. ${ }^{1}$; Massenkeil, G. ${ }^{2}$

${ }^{1}$ Medizinische Klinik m. S. Hämatologie/Onkologie, Charité

Universitätsmedizin Berlin, Berlin, Germany, ${ }^{2}$ Medizinische Klinik 2 ,

Städtisches Klinikum Gütersloh, Hämtologie/Onkologie, Gütersloh, Germany

In the GMALL Study, high risk and very high risk ALL patients (pts) are candidates for allogeneic stem cell transplantation (SCT) in first complete remission (CR1). Between 1995 and 201085 ALL patients in CR1 were transplanted in our center. Median age was 36 years (17 - 67). 28/85 patients had very high risk criteria $(\mathrm{Ph}+)$ and $57 / 85$ pts had high risk criteria. Stem cell donors were HLA identical siblings in 37/85 pts and HLA compatible unrelated donors in 48/85 pts. Up to the age of 55 in the GMALL Study protocol conditioning therapy is planned to be 12 Gy TBI and VP16 or cyclophosphamide (CY), pts with contraindications against TBI will receive reduced intensity conditioning (RIC) as do pts $>55$ years. In our patient group 80/85 pts were $\leq 55$ years and $5 / 85$ pts $>55$ years. $74 / 80$ pts received standard conditioning with 12 Gy TBI and $120 \mathrm{mg} / \mathrm{kg}$ CY or $60 \mathrm{mg} / \mathrm{kg}$ VP16, $5 / 80 \mathrm{pts}$ received RIC (FLU/BU/ATG) because of fungal pneumonia (3), cerebral toxicity (1) and 60 Gy irradiation because of glioblastoma (1), 1/80 pts received Treosulfan conditioning because of irradiation of an thyroid carcinoma. 5/85 pts were $>55$ years and received RIC.

Results: With a median follow up of 51 months (2 - 144) 48/85 pts are alive in complete remission (CCR). Probability of survival for the whole group at 10 years is 0.45 . Probability of survival for TBI conditioning $(n=74)$ was 0.46 at 10 years and for RIC $(n=10) 0.29$ at 8 years $(\mathrm{p} 0.823)$. In the TBI group, $11 / 74$ pts $(15 \%)$ relapsed, $21 / 74$ pts ( $28 \%$ ) died due to TRM. In the RIC group 4/10 pts relapsed, $1 / 10$ pts died due to TRM. We analyzed if survival in the TBI group is influenced by age. The age group $17-29$ years $(\mathrm{n}=29)$ had a probability of survival at 10 years of 0.45 , age $30-39$ years $(n=17)$ at 7 years of 0.69 and age group $40-55$ years $(n=28)$ at 10 years of $0.40(p 0.327)$. $3 / 5$ patients $>55$ years are alive in CCR, 2/5 died due to relapse.

In conclusion: In pts $\leq 55$ years standard conditioning with 12 Gy TBI and VP16 or CY results in long term survival of about $50 \%$. The relapse rate is low (15\%), the TRM of $30 \%$ is not age dependent. An improved prophylaxis and therapy of infections and GvHD may decrease TRM. 12 Gy TBI conditioning can be used in HR and VHR ALL pts up to 55 years for an effective leukemia control.

Disclosure: No conflict of interest disclosed.

\section{Wissenschaftliches Symposium Metastasierung}

\section{V714}

\section{MACC1, a newly identified regulator of HGF-MET} signaling, predicts metastasis in colorectal cancer

Stein, U. ${ }^{1}$; Arlt, F. ${ }^{1}$; Schwabe, H. ${ }^{2}$; Walther, W. ${ }^{1}$; Smith, J. ${ }^{3}$; Sack, U. ${ }^{3}$; Fichtner, I. ${ }^{3}$; Birchmeier, W. ${ }^{3}$; Schlag, P.M. ${ }^{4}$

${ }^{1}$ Experimental and Clinical Research Center, Charité Universitätsmedizin Berlin, am Max-Delbrück-Centrum für Molekulare Medizin, Berlin, Germany, ${ }^{2}$ Robert-Rössle-Klinik, Charité Universitätsmedizin Berlin, Berlin, Germany, ${ }^{3}$ Max- Delbrück-Centrum für Molekulare Medizin, Berlin, Germany, ${ }^{4}$ Charité Comprehensive Cancer Center, Berlin, Germany

Introduction: Currently used clinical and histopathological criteria of colorectal cancer are not sufficient for an early identification of subjects at highrisk for metastasis. Metastasis, however, is the most frequent cause of treatment failure and the most lethal attribute of colorectal cancer. Therefore, we searched for unknown genes that might be useful as prognostic indicators and intervention targets for distant colon cancer metastasis. Here we report on the identification and characterization of a novel gene, "Metastasis-Associated in Colon Cancer 1", MACC1.

Methods: We identified the previously undescribed gene MACC1 by genomewide expression analysis in primary and metastatic carcinomas of colon cancer patients. We evaluated MACC1 expression in tissues of colon cancer 
patients with respect to metastasis and survival. We performed detailed experimental in vitro and in vivo studies, as well as translational studies to identify the impact of MACC1 for cell motility and metastasis.

Results: Based on MACC1 expression in not metastasized primary colon cancers, our negative and positive prediction for metachronous metastasis was correct to $80 \%$ and $74 \%$, respectively. The 5-year-survival was $80 \%$ for MACC1 low expressors, but $15 \%$ for individuals with high MACC1 expression in the primary tumors. MACC1 expression in primary tumor specimens is a useful classifier for patients with low- vs high-risk of tumor metastasis. MACC1 induces proliferation, colony formation, migration, invasion, wound healing, and hepatocyte growth factor (HGF)-triggered scattering in cell culture. MACC1 acts as key regulator of the HGF/Met signaling pathway. We identified the HGF receptor gene, Met, as a transcriptional target of MACC1. Importantly, MACC1 acts as an inducer of tumor growth and metastasis in xenograft models. Knock down of MACC1 by RNAi technology, by key molecule inhibitors of MACC1-regulating upstream pathways, and by approaches interdicting its $\mathrm{SH} 3$ domain led to reduction of metastasis formation in mice, as demonstrated by end point measurements and by in vivo imaging.

Conclusion: MACC1 is a prognostic indicator for metastasis formation and metastasis-free survival, independent of the currently used tumor staging. MACC1 represents a promising new target for intervention of metastasis formation.

Disclosure: No conflict of interest disclosed.

\section{Expertenseminar}

Schilddrüsenkarzinom

\section{V718}

\section{Targeted therapy for patients with thyroid cancer}

Schütt, $P^{1}{ }^{1} ;$ Eberhardt, $W^{1}$

${ }^{1}$ Universitätsklinikum Essen, Innere Klinik (Tumorforschung), Essen, Germany

Introduction: The therapeutic options for metastatic and radioiodine-refrac tory differentiated and/or medullary thyroid cancer are rare and the benefit from classical chemotherapeutic drugs in this situation is limited. In recen years huge advantages have been made in understanding the molecular pathways that are involved in the pathogenesis of this disease. Based on this knowledge multiple novel agents targeting kinases involved in the pathogenesis of thyroid cancer have entered clinical trials.

Material and methods: A search of the Medline and ASCO databases was conducted to identify results of clinical trials using targeted therapy for thyroid cancer.

Results: Sorafenib, sunitinib, motesanib, vandetanib, and axitinib have been investigated in phase II trials in patients with differentiated and/or medullary thyroid cancer. In these studies significant clinical activity was observed with objective response rates of up to $35 \%$ and disease control rates $(\mathrm{CR}+\mathrm{PR}+\mathrm{SD})$ of up to $80 \%$ of patients. Median progression-free survival ranges from 7-28 months. Adverse events were mostly mild to moderate and comparable to studies of these drugs in other malignancies.

Conclusion: These results demonstrate that several drugs have significant clinical activity in patients with differentiated and/or medullary thyroid cancer and therefore further investigation of these agents in phase III trials is warranted.

Disclosure: No conflict of interest disclosed.

\section{Wissenschaftliches Symposium \\ GvHD and infectious complications after SCT: Trends and perspectives in pathogenesis and therapy \\ V719 \\ Current challenges in chronic GvHD}

Socie, G. $^{1}$

${ }^{1}$ Hospital Saint Louis, Hematology, Paris, France

Chronic graft-versus-host disease (GvHD), a multi organ disorder, is the leading cause of late non - relapse mortality after HSCT. Despite many years of experience we are still faced with numerous challenges including; lack of reli- able pre-clinical models, poor knowledge of human pathophysiology, validated diagnostic and severity criteria, and unpredictable clinical response to first line treatment.

In contrast to acute GVHD, chronic GVHD often presents with clinical manifestations that resemble those of autoimmune diseases. Nevertheless, relatively little is know about the immune mechanisms that lead to the development of chronic GVHD and how these might be similar or different than conventional autoimmune diseases. Both acute and chronic GVHD can be prevented by depletion of $\mathrm{T}$ cells from the donor graft. Thus, donor $\mathrm{T}$ cells responding to allogeneic antigens in the recipient must play a central role in the development of chronic GVHD, but the precise immunologic targets of chronic GVHD are not well established and the immunologic mechanisms that lead to the distinctive but highly variable clinical features of this disease remain largely unknown. Recent studies have provided evidence that B cells also contribute to the clinical manifestations of this disease.

Clinical syndromes with features of typical acute GVHD are increasingly recognized beyond 100 days after HSCT. In addition, patients with acute GvHD may progress to develop chronic GvHD with symptoms of both acute and chronic GVHD. The NIH consensus proposed standard criteria for diagnosis organ scoring and global assessment of chronic GvHD severity.

In general, systemic immunosuppressive treatment is not needed for patients with mild manifestations of chronic GVHD involving a single organ, unless adverse risk factors are present. Risk factors associated with an increased risk of non-relapse mortality include platelet count $<100,000$ at onset, treatment with prednisone at onset, antecedent acute GVHD, and hyperbilirubinemia at onset. Systemic treatment is needed for patients with more severe manifestations, especially when multiple sites are involved. The treatments studied during the past two decades, have not demonstrably accelerated the resolution of chronic GVHD. Survival at 5 years among patients with "standard-risk" chronic GVHD is approximately 70\%, and survival among those with "highrisk" features of thrombocytopenia or antecedent acute GVHD is approximately $50 \%$.

Disclosure: No conflict of interest disclosed.

\section{V720}

\section{Cellular therapy of viral infection} Riddell, S. ${ }^{1}$; Gardner, R. ${ }^{1}$; Reik, A. ${ }^{2}$; Holmes, M. ${ }^{2}$; Gregory, P. ${ }^{2}$;
Jensen, M.

${ }^{1}$ Fred Hutchinson Cancer Research Center, Seattle, United States, ${ }^{2}$ Sangamo Biosciences, San Francisco, United States, ${ }^{3}$ Seattle Children's Hospital, Seattle, United States

Introduction: The reactivation of endogenous latent viruses such as cytomegalovirus, continue to contribute to significant morbidity and mortality after allogeneic SCT. Progressive infection results primarily from deficient CMVspecific $\mathrm{T}$ cell immunity, either as a consequence of insufficient numbers of virus-specific $\mathrm{T}$ cells or of qualitative defects in $\mathrm{T}$ cell function resulting from inhibition by immunosuppressive drugs administered to prevent or treat graft versus host disease (GVHD). The adoptive transfer of CMV-specific T cells can restore deficient responses and is effective in a subset of patients. However, glucocorticoids, which are routinely administered to treat GVHD and have been associated with the greatest risk of severe CMV disease, suppress both endogenous and adoptively transferred $\mathrm{T}$ cells and interfere with the efficacy of $\mathrm{T}$ cell therapy. Thus, strategies to render virus-specific $\mathrm{T}$ cells selectively resistant to glucocorticoids may be of benefit for improving adoptive therapy. Methods: We investigated interfering with glucocorticoid receptor function in $\mathrm{T}$ cells using gene editing with zinc finger nucleases (ZFNs) that target the GR locus for cleavage. Primary CMV-specific T cells were subjected to editing of the GR gene and GR knockout (GR-/-) T cell clones were derived and characterized for specificity, function and glucocorticoid resistance.

Results: ZFNs delivered by an adenoviral vector provided efficient cleavage of the GR locus in CMV-specific T cells, and homozygous GR-/- T cells were readily isolated. GR-/- T cells grew equivalently well to wild-type T cells and were equally capable of lysing CMV-infected target cells and producing ifn gamma and TNF-alpha. Dexamethasone induced apoptosis of wild-type T cells and inhibited proliferation and cytokine production in response to CMV antigen. By contrast, GR-/- cells survived in the presence of dexamethasone were completely protected from the suppressive effects of dexamethasone on $\mathrm{T}$ cell function. 
Conclusions: Adoptive $\mathrm{T}$ cell therapy is an advancing modality for restoring $\mathrm{T}$ cell responses in immunodeficient SCT recipients. The subset of patients that require intense treatment with glucocorticoids for GVHD are unlikely to benefit from therapy because both transferred $\mathrm{T}$ cells are subject to suppression. Gene editing with ZFNs to render CMV-specific T cells selectively resistant to steroids provides a potential strategy to provide effective therapy in highly immunosuppressed patients.

Disclosure: No conflict of interest disclosed.

\section{V722 \\ Neovascularisation as a new therapeutic target for graft- versus-host disease therapy}

Penack, $0 .^{1}$

${ }^{1}$ Charité Campus Benjamin Franklin, Med. Klinik III, Hämatologie und Onkologie, Berlin, Germany

Allogeneic hematopoietic stem cell transplantation (allo-HSCT) is an important therapy with curative potential for a variety of malignant and non-malignant diseases. The major obstacles to a more favorable therapeutic outcome are tumor relapse and acute graft-versus-host disease (GVHD).

Neovascularization is the new formation of blood vessels in adults. Recent research linked neovascularization to both tumor growth and to inflammation making neovascularization an attractive therapeutic target in patients with malignancies undergoing allo-HSCT.

There is an increasing amount of interesting data showing that neovascularization plays an important role in GVHD and in tumor growth after allo-HSCT The scope of this talk is to summarize studies on neovascularization during allo-HSCT and to discuss clinical significance as well as implications for future therapeutic strategies.

Disclosure: No conflict of interest disclosed.

\section{Freie Vorträge \\ AML experimentell 2 \\ V723 \\ Analysis of mitosis in acute myeloid leukemia using live- cell imaging}

\section{Schnerch, D.M. ${ }^{1}$; Felthaus, J. ${ }^{1}$; Engelhardt, M. ${ }^{1}$; Wäsch, R. ${ }^{1}$} ${ }^{1}$ Uniklinik Freiburg, Hämatologie / Onkologie, Freiburg, Germany

Introduction: An impaired mitosis leads to genetic instability and bears the potential to drive tumor growth. The anaphase-promoting complex/cyclosome (APC/C) is an important ubiquitin ligase that is involved in mitotic control. Its activating subunit $\mathrm{Cdc} 20$ mediates ubiquitination of mitotic regulators such as Cyclin B and Securin which promotes the transition from metaphase to anaphase. APC ${ }^{\mathrm{Cdc} 20}$-dependent proteolysis is controlled by the spindle assembly checkpoint (SAC). The SAC senses improper chromosome orientation, tension across the kinetochor and the presence of unattached chromosomes. Holding back cells at metaphase prevents an unequal distribution of chromosomes onto the two developing daughter cells and is important to guarantee genetic integrity.

Methods/results: We compared lymphoblastic and myeloblastic cell lines and found myeloid cells to be deficient of arresting at metaphase in the presence of the microtubule-disrupting agent nocodazole. The mitotic checkpoint protein BubR1 was found to be downregulated in the majority of the investigated AML cell lines. Primary patient derived AML blasts also exhibited BubR1 expression at much lower levels. It is generally accepted that BubR1 knockdown causes genetic instability and leads to a significant shortening of the time from nuclear envelope breakdown to anaphase onset. We applied live-cell imaging to exactly characterize mitotic timing in leukemia cells. The expression of a GFP-tagged derivative of histone $\mathrm{H} 2$ served to visualize chromosomes in living cells. We could find a significant shortening of mitosis in the myeloblastic cell line Kasumi-1 cells $\left(\sim 30^{\prime}\right)$ when compared to the lymphoblastic cell line DG-75 ( 46’). To clarify whether BubR1 repression leads to a faster, potentially premature, passage through mitosis, we aim at overexpressing BubR1 in AML to revert the repression phenotype. Furthermore, visualization of chromatin by expression of histone H2-GFP has also proved useful to address the frequency of different features of genetic instability, such as lagging chromosomes or anaphase bridges.

Conclusion: As BubR1 is frequently repressed in AML it might be an important player in leukemogenesis. This hypothesis is underlined by the shortened time to anaphase in Kasumi- 1 cells, which is also known as a characteristic of BubR1-depletion. Mitotic regulators are already targets in AML therapy and a deeper understanding of mitotic processes in AML might lead to improved treatment approaches.

Disclosure: No conflict of interest disclosed.

\section{V724 \\ Transmigration of leukemic blasts after CXCL12 mediated activation of the CXCR4-PI3K-AKT pathway is disrupted by the dual mTOR/PI3K inhibitor NVP-BEZ235}

\author{
Kampa-Schittenhelm, K.M. ${ }^{1}$; Kanz, L. ${ }^{1}$; Schittenhelm, M.M. ${ }^{1}$ \\ ${ }^{1}$ Universitätsklinikum Tübingen, Med. II Hämatologie, Onkologie, \\ Rheumatologie, Immunologie, Pulmologie, Tübingen, Germany
}

Introduction: Acute myeloid leukemia (AML) remains difficult to treat. Patients typically respond initially to treatment with anthracycline and cytarabine-based induction chemotherapy, but most patients ultimately relapse and die of the disease. We hypothesize that the bone marrow (BM) environment has a protective influence on leukemic blasts during chemotherapy which is mediated by growth factors produced or expressed by stromal cells. CXCR4 is well characterized for its role in migration of hematopoietic progenitor cells, and we previously reported that CXCR4 is also expressed on the surface of AML blasts located in the BM. CXCR4 activates AKT-MAPK and G-protein coupled pathways, and there is evidence that CXCR4 inhibition mobilizes leukemic blasts to the peripheral blood causing sensitization to chemotherapy. Mobilization is not exclusive to leukemic blasts, but also affects hematopoietic stem cells. This may activate CXCR4 mediated pathways resulting in increased proliferation and thus result in higher susceptibility to chemotherapy causing unforeseen long term toxicity and hematopoietic insufficiency.

Methods: We performed immunophenotyping for CXCR4 and phospho AKT on BM blasts of 45 patients with AML as well as on hematopoietic stem cells (HSC) of healthy donors. Transmigration assays of ex vivo patient AML blasts and HSC were performed in presence as well as absence of the novel dual PI3K/mTOR inhibitor NVP-BEZ235.

Results: Immunophenptyping of AML patient BM revealed high levels of CXCR4 as well as high intracellular expression levels of AKT phosphorylation at codon 473 as well as codon 308 . In contrast, CXCR4 expression and phosphorylation of AKT was lower in healthy HSC. Inhibition of the PI3K-AKT signaling pathway by NVP-BEZ235 led to disruption of the transmigratory capacity of leukemia cells towards the CXCR4 ligand CXCL12 (SDF-1), while this effect was less pronounced in HSC. The anti-transmigratory effect was thereby comparable with the effect of the CXCR4 antagonist AMD3100. Most importantly and in contrast to AMD3100, NVP-BEZ235 was found to have additional antitumor efficacy with induction of apoptosis in the nanomolar range.

Conclusion: PI3K-inhibitors such as NVP-BEZ235, which is currently under clinical investigation for a variety of tumors, may be attractive agents in leukemia to overcome disease resistance in the BM.

Disclosure: No conflict of interest disclosed.

\section{V725}

\section{High expression of IGFBP2 in adult acute myeloid leukemia and association with drug resistance}

Kühnl, A. ${ }^{1}$; Kaiser, M. ${ }^{1}$; Heesch, S. ${ }^{1}$; Thiel, E. ${ }^{1}$; Baldus, C. ${ }^{1}$

${ }^{1}$ Charité Berlin Campus Benjamin Franklin, Berlin, Germany

Introduction: Overexpression of the gene IGF Binding Protein (IGFBP) 2 and high serum levels of the IGFBP2 protein have been described as adverse prognostic factors in pediatric acute myeloid leukemia (AML) and acute lymphoblastic leukemia (ALL). Here, we have investigated the expression pattern and prognostic significance of IGFBP2 in adult AML.

Methods: IGFBP2 mRNA expression was determined by quantitative realtime PCR in pretreatment bone marrow (BM) samples of 101 adults with 
newly diagnosed AML (median age 58 years), treated between 2003-2010 with standard induction chemotherapy in our institution. For statistical analyses, patients were divided into quartiles according to IGFBP2 expression levels [Q1-3: low IGFBP2 ( $\mathrm{n}=77), \mathrm{Q} 4$ : high $\operatorname{IGFBP2}(\mathrm{n}=24)]$.

In addition, IGFBP 2 mRNA was determined in the same patient cohort in peripheral blood samples (PB; $n=16)$ and serum IGFBP2 protein levels were measured by ELISA $(n=23)$. Healthy donors were used as controls (BM: $n=8$; PB: $n=6$; serum: $n=3$ ).

Results: $I G F B P 2$ mRNA expression was significantly higher in $\mathrm{BM}(P=0.037)$ and $\mathrm{PB}(P=0.036)$ samples of AML patients compared to healthy controls. Moreover, AML patients showed a significantly increased IGFBP2 serum protein level compared to controls $(P=0.002)$. IGFBP2 BM mRNA and serum protein levels were positively correlated $(\mathrm{r}=0.6 ; P=0.007)$.

Low BM IGFBP2 mRNA expression was associated with a myelomonocytic FAB subtype, whereas no other differences were found regarding clinical or genetic characteristics between the IGFBP2 expression groups. High IGFBP2 expression was significantly associated with a higher incidence of primary resistant disease $(I G F B P 2$ high $63 \%, I G F B P 2$ low $33 \% ; P=0.03)$ and with a lower complete remission (CR) rate (IGFBP2 high 30\%, IGFBP2 low 57\%; $P=0.03$ ). In multivariate analysis, high $I G F B P 2$ expression was independently predictive for therapy resistance [OR 6.2 (95\% CI 1.2-31.4); $P=0.03$ ] and achievement of CR [OR 0.2 (95\% CI 0.04-1.0); $P=0.05$ ]. No differences in overall or relapse-free survival were seen according to $I G F B P 2$ expression. Conclusions: IGFBP2 is aberrantly expressed in adult AML patients, with high $I G F B P 2$ expression predicting an unfavourable response to induction therapy. Our findings support the role of autocrine IGF signaling in proliferation and chemoresistance of AML cells. AML patients with an activated IGF pathway may potentially benefit from new therapies targeting the IGF axis.

Disclosure: No conflict of interest disclosed.

\section{V726}

\section{FLT3-ITD signaling induces oncogenic microRNA - 155 in acute myeloid leukaemia}

\section{Gerloff, D. ${ }^{1}$; Bräuer, D. ${ }^{1}$; Behre, G. ${ }^{1}$}

${ }^{1}$ Martin-Luther-Universität, Universitätsklinikum Halle(Saale), Klinik für Innere Medizin IV, Landeszentrum für Zell- und Gentherapie, Halle, Germany

Introduction: Almost $30 \%$ of all acute myeloid leukaemias (AML) are associated with an internal tandem duplication (ITD) in the juxtamembrane of FLT3 This mutation activates differential signaling in the FLT3 regulated pathways, for example STAT5, compared to FLT3 wildtype. Dysregulation of pathway activation causes a differentiation block and may play a role in inhibition of hematopoietic transcription factors like PU.1 and C/EBPalpha. MicroRNA (miRNA) expression is also targeted by FLT3-ITD signaling. MiRNAs are small (19 - $22 \mathrm{nt})$ non coding RNAs, which regulate protein expression by binding to a 3'UTR of their target mRNAs. MicroRNA-155 (miR-155) was found to be upregulated in FLT3/ITD associated AML patient samples.

Results: MicroRNA microarray analysis shows a decreased miR-155 expres sion during MCSF induced CD34+ stem cell differentiation into monocytes. We demonstrate that miR-155 overexpression in PMA treated U937 cells reduces macrophage differentiation. Further we could show miR-155 is FLT3ITD mediated, so a block of FLT3-ITD tyrosine kinase activity in MV4;11 cells via protein kinase inhibitor, leads to lower ( 0.2 fold) miR-155 expression, while overexpression of FLT3-ITD in U937 cells results in 2.5 fold induction of miR-155. A very famous component of FLT3-ITD signaling pathway is STAT5. Autophosphorylation of FLT3-ITD leads to constitutive activation of STAT5. In our further investigations we could prove that a knockdown of STAT5 by siRNA overcomes the FLT3-ITD induced miR-155 expression in MV4;11 cells. Another regulator for miR-155 is NF-kB (p65). Here we also show that a siRNA knockdown of p65 decreases miR-155 expression to 0.7 fold. Further we recovered that a siRNA block of STAT5 or p65 overcomes FLT3-ITD induced miR-155 expression. In ChIP analyses we found that NF-kB (p65) binds to the miR-155 promotor in MV4;11 cells.

Conclusion: All together we hypothesis, that FLT3-ITD induces the oncogenic miR-155 via STAT5 and NF- $\mathrm{BB}$ (p65). The increased miR-155 expression enhances the myeloproliferative disorder in acute myeloid blasts. These data reveal the impact of deregulated miRNAs in cancer development and may provide novel biomarkers and therapeutic targets in leukaemia.
Disclosure: No conflict of interest disclosed.

\section{V727}

\section{Association of HLA-G haplotype risk model with overall survival in acute myeloid leukemia patients}

\section{Volbracht, A. ${ }^{1,2}$; Novotny, J. ${ }^{1}$; Ochsenfarth, C. ${ }^{3}$; Switala, M. ${ }^{2}$;} Frey, U. ${ }^{4}$; Horn, P. ${ }^{2}$; Dührsen, U. ${ }^{1}$; Rebmann, V. ${ }^{2}$; Nückel, H. $^{1}$

${ }^{1}$ Universitätsklinik Essen, Klinik für Hämatologie, Essen, Germany,

${ }^{2}$ Universitätsklinik Essen, Institut für Transfusionsmedizin, Essen, Germany, ${ }^{3}$ Universitätsklinik Essen, Institut für Pharmakogenetik, Essen, Germany, ${ }^{4}$ Universitätsklinik Essen, Klinik für Anästhesie und Intensivmedizin, Essen, Germany

Introduction: The human leukocyte antigen G (HLA-G) molecule exhibits limited tissue distribution and exerts multiple immunosuppressive functions. Thus, HLA-G expression in tumor cells favors tumor immune escape and tumor progression. The HLA-G gene polymorphism is extremely restricted in comparison to the classical HLA antigens. However, specific HLA-G polymorphisms in exons 3, 4, 5, 7 and parts of the 3'UTR encode for different haplotypes/alleles. Because of the prognostic role of HLA-G in cancer, especially in B-CLL, we investigated here the role of the HLA-G haplotypes in acute myeloid leukemia (AML).

Methods: Genotyping was performed on the basis of examining exons 3, 4, 5, 7 and parts of the 3'UTR by pyrosequencing in 166 patients with AML. In total, we found 17 different haplotypes in the cohort.

Results: The haplotype distributions between 166 AML patients and 190 healthy controls were significantly different in two alleles, arguing that these two haplotypes of HLA-G on the one hand increases $(* 01: 06, p=0.042)$ and on the other hand decreases $(* 01: 01: 12$ variante, $\mathrm{p}=0.0014)$ the susceptibility for AML. Next a risk model was generated for overall survival (OS) adapted to specific haplotypes/alleles of the HLA-G gene. The favorable group (A) consisted of the alleles $* 01: 06$ and $* 01: 01: 20$ variant (12 patients), the intermediate group (B) of *01:01:20, *01:04:10, *01:01:30 and *01:01:41 variant (113 patients) and the unfavorable group (C) of the remaining 11 alleles (41 patients). The OS for patients in group $\mathrm{C}$ was significantly $(\mathrm{p}=0.02)$ shorter (median OS 613 days) than for those in group B (median OS 961 days) and A (median OS not reached). Multivariate analysis shows a trend that the risk HLA-G model (Hazard ratio $(\mathrm{HR}) 1.4, \mathrm{p}=0.084$ ) was an independent predictor next to the established prognostic factors cytogenetics (HR 2.1, p=0.001), age (HR 1.8, $\mathrm{p}=0.009)$, leucocytes (HR 1.6, p=0.026), ECOG stage (HR 1.5, p=0.006) and platelets (HR 2.1, $\mathrm{p}=0.015$ ). Moreover, the new risk model was able to further subdivide patients with intermediate cytogenetic risk profile (median OS: group A not reached; group B 987 days; group C 624 days; $\mathrm{p}=0.10$ ).

Conclusion: Our study is the first study demonstrating that the combination of different alleles of the HLA-G gene is associated with the overall survival in AML patients. This fact emphasizes the extensive role of this gene by the tumor escape mechanism and could be responsible for progress in other cancers.

Disclosure: No conflict of interest disclosed.

\section{V728}

KIT inhibition upregulates heat shock proteins - a potential mechanism of resistance to KIT tyrosine kinase inhibitors

\section{Grandl, T. ${ }^{1}$; Mueller, S. ${ }^{1} ;$ Kanz, L. ${ }^{1}$; Schittenhelm, M.M. ${ }^{1}$; Kampa-} Schittenhelm, K.M.

${ }^{1}$ Universitätsklinikum Tübingen, Med. II Hämatologie, Onkologie, Rheumatologie, Immunologie, Pulmologie, Tübingen, Germany

Introduction: Activating mutations of the KIT class III receptor tyrosine kinases (TK) are associated with many human cancers, including core binding factor leukemias (CBF AML) and systemic mastocytosis (SM). Despite the encouraging therapeutic potential of KIT-TK inhibitors (TKI), resistance leading to disease progression occurs in many patients, specifically after TKI monotherapy. We hypothesized that resistance to therapy is promoted by activation of alternative signaling pathways which override TKI inhibition. 
Methods: To explore the downstream signaling pathways of class III receptor TK, we performed unbiased phoshoproteomic analyses of leukemia and mastocytosis cell lines before and after TKI treatment. Phosphopeptides that displayed a significant difference in phosphorylation before and after KIT-inhibition with Imatinib at IC90 $(100 \mathrm{nM})$ were immunoaffinity purified and identified by tandem mass spectrometry. Differential expression of identified proteins was verified by qRT-PCR and Western Blot. Antiproliferative and proapoptotic effects of the heatshock protein (HSP90) inhibitor IPI-504, were studied in our isogenic Baf/3 KIT cell model harboring different clinically relevant KIT mutations alone and in combination with different TKI.

Results: Phoshoproteomics revealed a significant upregulation of phosphorylation levels of peptides identified as members of the HSP family in KIT cel lines. HSP upregulation after inhibition with the TKIs Imatinib and Dasatinib was confirmed by Western blotting in a variety of mutant-KIT hematopoietic cell line models including leukemia and mastocytosis. Depending on the KIT isoform, IPI-504 potently inhibited proliferation and induced apoptosis with an IC50 as low as 500nM. Tantalizingly, combination of IPI-504 with TKIs resulted in a dramatic potentiation of the antiproliferative and proapoptotic effects achieved by either drug alone. Antitumor efficacy in combination therapy was observed even at HSP90 inhibitor concentrations that did not display antitumor activity if administered alone.

Conclusion: Our model suggests that inhibition of KIT causes upregulation of heat shock proteins serving to stabilize the functionality of targeted autoactivated receptor TK, which provides a potential mechanism for resistance to TKI therapy. Importantly, we provide a rationale to combine TKI with (lowdose) HSP-inhibitors such as IPI-504 to optimize TKI therapy.

Disclosure: No conflict of interest disclosed.

\section{Freie Vorträge \\ Stammzellbiologie}

\section{V729}

Homing, proliferation and mobilization of hematopoietic stem cells is regulated through the interaction of $\alpha_{9} \beta_{1}$ and Osteopontin

Grassinger, J.'; Storan, M.2; Williams, B. ${ }^{2}$; Olsen, G. ${ }^{2}$; Haylock, D.N. ${ }^{2}$; Nilsson, S.K. ${ }^{2}$

${ }^{1}$ University of Regensburg Medical Center, Department of Hematology and Oncology, Regensburg, Germany, ${ }^{2}$ Commonwealth Scientific and Industrial Research Organisation (CSIRO), Molecular and Health Technologies, Clayton, Australia

Introduction: There is significant evidence that hematopoietic stem cells (HSC) reside in a specialized microenvironment ("niche") within the bone marrow (BM) of adult mammals that regulates HSC fate through cell-cell contact, cell-stroma contact and soluble factors. This point of view is strongly supported by the fact that osteoblasts, located within the endosteal BM region, play a key role in homing, proliferation and mobilization of HSC. In this study we provide new insights into the regulatory process within the endosteal stem cell niche.

Methods: In order to further understand the interaction between HSC with the BM microenvironment, a series of molecular and functional experiments, involving knockout mouse models, were conducted.

Results: Transplantation experiments into C57BL/6 mice revealed that murine HSC, defined as CD150+CD48-LSK cells, preferentially lodge within the endosteal BM within 15 hours. This is of importance as we have previously demonstrated that HSC isolated from the endosteal BM region have significantly increased biological potential compared to HSC located in other regions of the BM. Osteopontin (OPN), a heavily phosphorylated glycoprotein expressed by osteoblasts at the endosteum, is already known as a negative regulator of the HSC pool size. In this study we now demonstrate that OPN is a potent chemo attractant for HSC and comparable to CXCL12. Moreover, mice devoid of OPN within the BM show significant endogenous and G-CSF responsive mobilization of $\mathrm{HSC}$ into the peripheral blood (PB), suggesting a key role for OPN in anchoring HSC within the niche. Molecular and functional analysis revealed that the $\mathrm{a}_{9} \mathrm{~b}_{1}$ integrin, a receptor for OPN, is expressed on murine and human HSC and acts as a key component in these processes. Murine HSC devoid of $a_{9} b_{1}$ show significantly reduced homing efficiency and engraftment potential in vivo. In addition, blocking $\alpha_{9} \beta_{1}$ on human HSC resulted in a significantly reduced migration ability in vitro.
Conclusion: In this study we demonstrated that the interaction of OPN with the $\alpha_{9} \beta_{1}$ integrin expressed on HSC plays a pivotal role in HSC regulation. A better understanding of the physiological processes within the BM stem cell niche provides the basis for the improvement of HSC transplantation and mobilisation. In addition, this will act as a model to gain further insights into the processes involved in leukemogenesis and tumour metastasis and may result in the discovery of new targets for cancer therapy.

Disclosure: No conflict of interest disclosed.

\section{V730}

The murine ecotropic viral integration site-1 (Evi-1) gene regulates myelopoiesis in zebrafish and human pluripotent stem cells

Konantz, M. ${ }^{1}$; Grauer, M. ; Brugman, M.H. ${ }^{2}$; Park, I.-H. ${ }^{3}$; Daley, G.Q. ${ }^{4,5}$; Nüsslein-Volhard, C. ${ }^{6}$ Kanz, L. ${ }^{1}$; Baum, C. ${ }^{7}$ : Lengerke, C. ${ }^{1}$

${ }^{1}$ Medizinische Klinik II, Tübingen, Germany, ${ }^{2}$ Hannover Medical School, Dept. Experimental Hematology, Hannover, Germany, ${ }^{3}$ Yale School of Medicine, Department of Genetics, Yale Stem Cell Center, New Haven, United States, ${ }^{4}$ Children`s Hospital Boston, Dept. Hematology\&Oncology, Boston, United States, ${ }^{5}$ Howard Hughes Medical Institute, Chevy Chase, United States, ${ }^{6}$ Max-Planck-Institut für Entwicklungsbiologie, Tübingen, Germany, ${ }^{7}$ Hannover Medical School, Hannover, Germany

Introduction: Reactivation of embryonic molecular pathways is often observed during adult oncogenic processes. The ecotropic viral integration site-1 (Evi-1) locus was originally identified as a common site of retroviral integration in murine myeloid tumors and was later shown to be one of the most potent oncogenenes associated with murine and human myeloid leukemia. While recent data suggest involvement of Evi-1 in embryonic hematopoiesis (Goyama et al, Cell Stem Cell 2008; Yuasa et al, EMBO J, 2005), the precise role and molecular regulation of Evi-1 during blood development yet remains poorly understood.

Methods: Hematopoietic development was analysed in zebrafish embryos by in situ hybridization for early hematopoietic markers ( $s c l$, gatal, pu.1, gata2, mpo, l-plastin) and analysis of gfp expression in globin and cmyb transgenic fish lines. Loss-of-function studies were performed in zebrafish embryo by injection of morpholino oligonucleotides inhibiting pre-mRNA splicing. To assess interspecies conservation, the effect of EVI-1 shRNA on differentiating human pluripotent stem cells was assessed by quantitative PCR, flow cytometry and functional CFU-assays.

Results: Double in situ hybridization studies showed co-localization of evi-1 expression with hematopoietic markers in zebrafish embryos. Morpholinoinjected embryo showed severely reduced numbers of circulating blood cells, due to a severe reduction of myelopoiesis (as documented by expression patterns of pu.1, gata2, mpo and l-plastin). Furthermore, cmyb reduction was observed suggesting impairment of definitive hematopoiesis. In contrast, no change was observed in early blood precursors in the posterior lateral plate mesoderm (as monitored by $s c l$ expression) or in primitive erythroid progenitor cells (as monitored by gatal expression). Overexpression of gata2 by mRNA co-injection in evi-1 morphants partially rescued the reduced myeloid phenotype implying gata2 as a downstream-target of evi- 1 during embryonic hematopoiesis. First results in human cells confirmed our observations obtained in the zebrafish model.

Conclusion: Our data indicate that evi-1 regulates zebrafish blood development and suggest specific involvement in the regulation of the myeloid lineage partially through activation of gata2. Currently ongoing experiments are investigating pathway conservation in human pluripotent stem cells and analyze other putative targets of EVI-1 during developmental hematopoiesis.

Disclosure: No conflict of interest disclosed. 
V731

\section{Cks1 controls the hematopoietic stem/progenitor cell compartment by regulating proliferation and apoptosis}

Nikolova, V.1; Graf, S.1; Pietschmann, E. ${ }^{1}$; Kratzat, S. ${ }^{1}$; Kröger, M. ${ }^{1}$; Quintanilla-Fend, L. ${ }^{2}$; Peschel, C. ${ }^{1}$; Oostendorp, R. ${ }^{1}$; Keller, U.

${ }^{1}$ Technische Universität München, III. Medizinische Klinik, München, Germany, ${ }^{2}$ Eberhard Karls Universität, Institut für Pathologie, Tübingen, Germany

The Cyclin-dependent kinase inhibitor (CKI) p $27^{\mathrm{Kip} 1}$ plays a pivotal role in the control of cell proliferation. p $27^{\mathrm{Kip} 1}$ has been shown to control hematopoietic stem cell repopulation efficiency. The control of $\mathrm{p} 27^{\mathrm{Kip} 1}$ protein involves $\mathrm{SCF}^{\mathrm{Skp} 2 / \mathrm{Cks} 1}$ ubiquitin ligase activity. Primary mouse fibroblasts derived from Cyclin-dependent kinase subunit 1-deficient $\left(\mathrm{Cks}^{-/-}\right)$embryos proliferate slower than wild type cells. Based on the role of CKI in hematopoiesis and Cks 1 function in regulating p $27^{\mathrm{Kip} 1}$ levels, we analyzed the stem cell compartment of $\mathrm{Cks}^{-1-}$ mice. We detected high Cks1 transcripts levels in the primitive CD34negative subset of LSK (Lin-Sca1+c-Kit+ cells). In accordance, we observed a significant decrease in multipotent hematopietic progenitor cells (MPP) and the LSK subset in $\mathrm{Cks}^{-/-}$mice. The deficiency of Cks1 in hematopoietic cells also resulted in decreased colony formation of whole bone marrow and lineagedepleted bone marrow cells. Ex vivo co-culture assays of wild type and $\mathrm{Cks}^{-1}$ lineage-depleted bone marrow on EL08 stromal cells confirmed the significant impairment of colony forming cells upon Cks1 loss. Since Cks1 is an important regulator of entry into $S$-phase, we studied whether the observed changes migh be due to deregulated cell cycle progression. In steady state hematopoiesis of 10-16 week old mice, the relative number of BrdU-positive cells was unchanged in $\mathrm{Cks}^{-/-}$mice. We next determined if $\mathrm{Cks} 1$ plays a role in regulating the response of HSC to conditions of stress. We analyzed the recovery of $\mathrm{Cks}^{-1}$ animals following perturbation of hematopoiesis with the hematotoxin $5 \mathrm{FU}$. The hematopoietic regeneration was severely disturbed in $\mathrm{Cks}^{-/-}$mice and only a minority of early hematopoietic cells (LSK and MPP) showed incorporation or BrdU. In order to further test the role of Cks1 in stress hematopoiesis we also performed transplantation of $\mathrm{Cks}^{-/-}$and wild type control bone marrow into lethally irradiated recipient mice. In addition to changes in proliferation and differentiation the observed impairment of the $\mathrm{Cks}^{-/-}$stem/progenitor cell compartment might also be due to increased apoptosis. Indeed, after ex vivo culture of Lineage-depleted $\mathrm{Cks}^{-/-}$and control cells cytokine withdrawal lead to a significant increase of apoptotic cells in the stem/progenitor cell subsets in the absence of Cks1. Our findings demonstrate that Cks1 is an important regulator of early hematopoiesis under various conditions.

Disclosure: No conflict of interest disclosed.

\section{V732}

\section{Natalizumab increases circulating CD34+ cells with an impaired functional potential in patients with multiple sclerosis. Bone marrow derived CD34+ cells remain unaffected}

Saure, C. ${ }^{1}$; Zohren, F. ; Bruns, I. ${ }^{1}$; Schroeder, T. ${ }^{1}$; Caddedu, R.P. ${ }^{1}$; Fischer, U. ${ }^{2}$; Kobbe, G. ${ }^{1}$; Hartung, H.P. ${ }^{3}$; Kieseier, B. ${ }^{3}$; Haas, R. ${ }^{1}$

${ }^{1}$ Uniklinik Düsseldorf, Hämatologie, Onkologie und Klinische Immunologie, Düsseldorf, Germany, ${ }^{2}$ Uniklinik Düsseldorf, Institut für molekulare Medizin, Düsseldorf, Germany, ${ }^{3}$ Uniklinik Düsseldorf, Neurologische Klinik,

Düsseldorf, Germany

Introduction: In our previous report (Zohren et al., 2008) we could show that the blockade of the heterodimer VLA-4 by the monoclonal IgG4 antibody natalizumab leads to a significant increase in circulating CD34+ cells in patients with multiple sclerosis (MS). We now extend our analysis on the influence of natalizumab on CD34+ cells, comparing bone marrow (BM) and peripheral blood $(\mathrm{PB})$ derived $\mathrm{CD} 34+$ cells of natalizumab patients with those from healthy donors.

Methods: A total of 83 patients with MS receiving natalizumab were included. In vitro adhesion, migration and apoptosis assays as well as LTC-IC of immunomagnetically enriched CD34+ cells were conducted. Flow cytomet- ric analyses were performed to assess phenotype and composition of the CD34+ subsets

Results: The median concentration of circulating CD34+ cells was significantly greater compared to normal volunteers $(7.7 / \mu \mathrm{L}$ vs. $1.9 / \mu \mathrm{L} ; \mathrm{p}=.0001)$ and remained constant as long as one year during treatment with natalizumab. Leukocyte cell counts, the number of $\mathrm{T}$ cell subsets as well as the number of CD19+ B cells and CD56+ natural killer cells were in normal range in PB and BM after short- and long-term treatment with natalizumab. However, we found significantly reduced adhesion and migration abilities of circulating CD34+ cells under natalizumab treatment in comparison to G-CSF mobilized CD34+ cells of healthy donors. Moreover, the self-renewal capacity of these cells was poor. In contrast, no significant difference was seen between the BM of natalizumab patients and the BM of healthy volunteers with regard to cellularity and proportion of CD34+ cells. In addition, neither co-expression of CD49d nor the adhesion ability of the BM derived CD34+ cells revealed a significant difference between the two collective.

Conclusions: The impaired functional potential of the circulating CD34+ cells as well as the uninfluenced BM CD34+ cells among natalizumab treatment argue against a mobilizing of the cells. Our data support the view of an impaired homing of the circulating CD34+ cells under natalizumab therapy.

Disclosure: No conflict of interest disclosed

\section{V733}

Mesenchymal stem cells form spheroid-like structures, which can serve as a 3D-model for the hematopoietic stem cell niche

\section{Wuchter, P. ${ }^{1}$; Saffrich, R. ${ }^{1}$; Straub, B. ${ }^{2}$; Ho, A.D. ${ }^{1}$}

${ }^{1}$ Universitätsklinikum Heidelberg, Abteilung Innere Medizin V, Heidelberg, Germany, ${ }^{2}$ Universitätsklinikum Heidelberg, Pathologisches Institut, Heidelberg, Germany

Introduction: Human mesenchymal stem cells (MSC) maintain "stemness" of hematopoietic stem cells (HSC) by cell-cell contact and junction formation when used as feeder-layer in coculture. We previously demonstrated the occurrence of complex cadherin-catenin-based junctions between HSC and MSC in this setting.

Methods: MSC were derived from bone marrow aspirates from healthy voluntary donors. MSC were seeded on a layer of $1 \%$ agarose gel in 96 well plates and after $24-48 \mathrm{~h}$ the formation of spheroid-like cell aggregations could be observed. This process was continuously monitored with time-lapse microscopy. MSC-spheroids were characterized by immunofluorescence microscopy and immunohistology. The presence of specific junction molecules was evaluated by western-blot. As a second step, MSC-spheroids were cocultured with HSC isolated from umbilical cord blood and cell migration was monitored with time-lapse microscopy. Using advanced confocal laser scanning microscopy in combination with deconvolution and volume rendering software, we generated 3D-images of intercellular junctions between HSC and MSC.

Results: Aggregation of MSCs resulted in spheroids consisting of about 500 cells. Formation of spheroids was independent upon different MSC culture conditions. Immunohistochemistry revealed that MSC-spheroids consist of homogeneously aggregated cells, which are positive for beta-catenin, $\mathrm{N}$-cadherin, vimentin and actin. The proliferation rate of MSCs measured by Ki-67-staining was under 5\%. Active cell-division activity was demonstrated by time-lapse microscopy. Upon coculture of HSCs with MSC-spheroids, HSCs showed directed locomotion towards MSC-spheroids. After 12h, several HSC connected to the outer cell layer of the MSC-spheroid. At the contact zone, cadherin-catenin-based junctions were identified.

Conclusions: We have demonstrated the surprising ability of MSC to form complex spheroid-like aggregates in vitro. Aiming to develop a more physiological 3D surrogate model for the hematopoietic stem cell niche, we analyzed migration pattern and junction-formation of HSC in a coculture setting with these MSC-spheroids.

Disclosure: No conflict of interest disclosed. 
V734

\section{Importance of CD44 and CD44v7 in the interaction of HPC and LSC with their Niche}

Taubert, I. ${ }^{1}$; Schubert, M. ${ }^{1}$; Hellwig, I. ${ }^{1}$; Zepeda-Moreno, A. ${ }^{1}$; Ran, D. ${ }^{1}$; Pietsch, L. ${ }^{1}$; Eckstein, V. ${ }^{1}$; Zöller, M. ${ }^{2} ;$ Ho, A.D. ${ }^{1}$

${ }^{1}$ Universitätsklinikum Heidelberg, Innere Medizin $V$, Stammzellforschung AG Ho, Heidelberg, Germany, ${ }^{2}$ Universitätsklinikum Heidelberg,

Experimentelle Chirurgie, Heidelberg, Germany

Introduction: The stem cell niche regulates maintenance of Hematopoietic Progenitor Cells (HPC) and Leukemic Stem Cells (LSC). The niche interaction of HPC and LSC is, amongst others, mediated by the multifunctional, ubiquitously expressed transmembrane protein CD44. Its maturation, apart from the standard isoform (CD44s), includes alternative splicing that allows the insertion of variable exons (v2-v10) in the extracellular region of the molecule. In this study, we compared the expression of CD44 variant isoforms (CD44v) in HPC and LSC and their importance in the interaction with mesenchymal stromal cells (MSC).

Methods: Mononuclear cells from patients with AML, mobilized periphera blood (mPB) and bone marrow (BM) from healthy donors or cord blood (CB) were sorted by flow cytometry for their CD34 fluorescence and or according to their ALDH activity. These subpopulations were analyzed for their CD44v expression on mRNA and protein level. Moreover CD34+ cells from CB were stained with CFSE and cultivated with or without MSC for 7 days and then analyzed for their CD34, CD11b and CD44v expression and for the amount of cell divisions. Using 96 well adhesion and flow chamber assays we investigated the effect of A3D8 and anti-CD44v7 on the interaction of HPC and KG-1a to MSC.

Results: There was a strong CD44s expression in all HPC and LSC subpopulations detectable compared to a relatively weak CD44v expression on mRNA and protein level. In HPC (ALDH+/CD34+) from CB and mPB almost no $\mathrm{CD} 44 \mathrm{v}$ expression could be measured in contrast to a low expression in HPC from BM. In $\mathrm{ALDH}+/ \mathrm{CD} 34+\mathrm{LSC}$ from patients with $\mathrm{AML}$ an upregulation of $\mathrm{CD} 44 \mathrm{v} 7$ was partly detectable. Ongoing proliferation and differentiation of HPC with or without MSC led to an increase in the CD44v expression, whereas the CD44v7 expression was higher when cultivated with MSC. These findings are consistent with the cell cycle dependant expression of CD44v found in KG-1a. Flow chamber assays with a defined shear stress showed a higher adhesion of HPC to MSC after incubation with A3D8, in contrast to a lower adhesion after incubation of A3D8 for 24 hours measured in 96 well assays without flow.

Conclusions: In this study we found differences in the expression of CD44v7 between HPC and LSC and an influence of CD44s and CD44v7 in the interaction with the stem cell niche, which could be important in the progression of novel therapeutic strategies in AML.

Disclosure: No conflict of interest disclosed.

Wissenschaftliches Symposium
Science Track
Tumorbiologie hämatologischer Neoplasien

V736

\section{Therapy-induced senescence improves treatment outcome in Myc-driven lymphomas in vivo}

Dörr, J.R. ${ }^{1} ; Y u, Y^{2}{ }^{2}$; Tabor, V. ${ }^{2}$; Loddenkemper, C. ${ }^{3}$; Dörken, B. ${ }^{1}$; Schmitt, C.A.

${ }^{1}$ Charité - Campus Virchow-Klinikum, Hämatologie, Onkologie und Tumorimmunologie, Berlin, Germany, ${ }^{2}$ Max-Delbrück-Center for Molecular Medicine, Berlin, Germany, ${ }^{3}$ Department of Pathology, Technical University Munich, Munich, Germany

Introduction: Premature senescence is a terminal G1 arrest in response to acute cellular stresses, such as oncogene activation or exposure to DNA damaging chemotherapy. Therefore, senescence complements apoptosis as a safeguard program and tumor-suppressive mechanism. However, no tumor model has shown that therapy-induced senescence (TIS) contributes to treatment outcome. Here, we use the Eu-myc mouse lymphoma model with and without intact alleles of the histone H3 lysine 9 (H3K9) methyltransferase
Suv39h1 (controlling senescence) and of p53 (mediating both apoptosis and senescence) to demonstrate a critical role for senescence in cancer therapy in vivo.

Methods: Lymphoma cells (LCs), with and without retrovirally transduced bcl 2 to block apoptosis, were treated with adriamycin in vitro or were exposed to cyclophosphamide upon lymphoma formation in immunocompetent mice in vivo. Apoptosis was assessed by AnnexinV/Propidium Iodide staining in vitro and TUNEL staining of lymph node sections in situ. TIS was analyzed by senescence-associated b-galactosidase activity (SA-b-gal), Ki67 staining and BrdU incorporation. Tumor formation, therapy and progression were monitored by whole-body fluorescence and luciferase imaging. Progressionfree and overall survival were evaluated using the Kaplan-Meier method. Minimal residual disease (MRD) in lymph node, spleen and bone marrow was detected by E $\mu$-myc specific, quantitative PCR.

Results: Although Suv39h1- lymphomas recapitulate the proliferation rate and sensitivity to drug-induced apoptosis of control (i.e. no further defined genetic defects) lymphomas, they display significantly shorter progressionfree and overall survival after chemotherapy in vivo. Nevertheless, Suv39h1deficient lymphomas display lower MRD levels than control lymphomas ten days after chemotherapy. However, only control LCs, but not Suv39h1- or p53-deficient LCs, enter TIS in vivo, as can be demonstrated particularly for bcl2-transduced LCs by uniform SA-b-gal reactivity. Therefore, control lymphomas maintain a higher tumor volume after therapy than Suv39h1- lymphomas due to senescence induction, but show improved disease control.

Conclusions: The study demonstrates that Suv39h1 acts as an essential mediator of TIS without compromising apoptosis or altering the proliferative capacity of LCs. Hence, TIS is a critical component of anticancer drug therapy in vivo and significantly extends progression-free and overall survival of the host.

Disclosure: No conflict of interest disclosed.

\section{V737}

\section{Biology of myeloid neoplasias}

\section{Bohlander, S.K. ${ }^{1}$}

${ }^{1}$ Universität München - Klinikum Großhadern - HelmholtzZentrum München, Medizinische Klinik und Poliklinik III, München, Germany

Myeloid malignancies result from the accumulation of somatic mutations in hematopoietic stem cells. These mutations lead to a proliferative advantage, a block in differentiation, reduced apoptosis and to other phenotypic changes of the malignant clone.

In myeloid malignancies, chromosomal translocations frequently result in the formation of fusion genes which often lead to the activation of tyrosine kinases (e.g. BCR/ABL, BCR/JAK2) or to the generation of an aberrant transcription factor (e.g. AML1/ETO, CBFB/MYH11). While it is well known that tyrosine kinase activation is associated with increased proliferation and that transcription factor fusions cause differentiation blocks, more complicated and less easily recognizable effects of fusion proteins might also be common. Thus we could recently show that the CALM/AF10 fusion protein does not only cause transcriptional deregulation of a large number of target genes but that it also leads to genome-wide histone H3 lysine 79 hypomethylation and increased genomic instability.

Point mutations also play an important role in myeloid malignancies. However, the number of genes that are known to be mutational targets is rather limited because almost all of these genes were identified as mutational targets serendipitously.

This situation has changed recently. With the advent of next generation sequencing it is now possible to screen entire genomes or transcriptomes for disease causing point mutations. The first of these analyses have shown that the mutational landscape of myeloid malignancies is rather complex and that between 3 and 8 genes are mutated in each case of AML. However, since many of the new mutational targets in AML seem to be rarely mutated in other cases, it is not easy to determine whether a mutaions is a driver of the malignant process or just a passenger. Thus, one of the challenges is to identify the true driver mutations in myeloid malignancies. To achieve a more comprehensive understanding of myeloid malignancies it will become necessary to combine genomic data with new functional assays and model systems. The collaborative research center "Molecular mechansims of normal and malignant hematopoiesis" (SFB 684) in Munich brings together physician scientists and basic researchers to approach these challenging questions. 
Disclosure: No conflict of interest disclosed.

\section{V738}

In vivo imaging in the individualized patient xenograft leukemia mouse model

Terziyska, N. ${ }^{1}$; Jeremias, I. $^{1,2}$

${ }^{1}$ Helmholtz Center München, Gene Vectors, München, Germany, ${ }^{2} \mathrm{Dr}$. von Haunersches Kinderspital, Hämatologie / Onkologie, München, Germany

The xenograft mouse model allows the propagation of individual patientderived leukemia cells in highly immune-compromised mice. Generation of new and even more severely immune-incompetent mouse strains facilitated the engraftment of human cells. NSG-mice represent NOD (non-obese diabetes) / SCID (severe combined immune deficiency) mice which additionally lack a functional gamma-chain of the IL-2 receptor and allow engraftment of both ALL and AML cells.

So far, this model could not be used for abundant preclinical therapy trials due to the inability to follow up development and therapy response of individual leukemias. Mice develop clinical or para-clinical signs of leukemias at very late time points and the distribution of leukemia in mice had to be measured posthumously and thus only once per mouse.

To bridge this gap, we established a new technique which allows in vivo imaging in individualized leukemias. We expressed luciferase in xenograft cells in order to perform bioluminescence in vivo imaging. First images show that the development of acute leukemia can be sensitively followed up by in vivo imaging in single animals over time.

Our new technique now enables the sensitive and continuous in vivo follow up of leukemia cells obtained from individual patients. Our new method allows individualized preclinical therapy trials for leukemia which might help to optimize future treatment of leukemia

Disclosure: No conflict of interest disclosed.

\section{Freie Vorträge \\ Science Track \\ Immuntherapie}

\section{V739}

Multi-tumor-antigen-specific cytotoxic T lymphocytes for therapy of hematologic malignancies

\section{Gerdemann, U. ${ }^{1}$; Vera, J.F.1; Tripic, T. ${ }^{1}$; Cruz, R.C. ${ }^{1}$; Heslop,} H.E. ${ }^{1}$; Brenner, M.K. ${ }^{1}$; Bollard, C.M. ${ }^{1}$; Rooney, C.M. ${ }^{1}$; Leen, A.M. ${ }^{1}$ ${ }^{1}$ Baylor College of Medicine, Center for Cell and Gene Therapy, Houston, United States

Introduction: Immunotherapy with EBV-specific cytotoxic T cells (CTLs) has been successfully used to treat EBV-associated tumors, including relapsed EBV+ Hodgkin lymphoma (HL). Though frequently effective, this approach has limited application since only approx. $50 \%$ of HL tumors are EBV+ve. To extend CTL therapy to all HL patients we have now developed a means of generating CTLs that recognize a spectrum of non-viral tumor-associated antigens (TAA) expressed by HL including Survivin, MAGEA4, and SSX2.

Methods: Autologous DCs pulsed with multiple TAA-pepmixes were used for PBMC stimulation. The whole antigen spanning pepmixes increase the range of suitable patient HLA polymorphisms, while targeting multiple antigens simultaneously reduces the risk of tumor immune evasion. We assessed the addition of different Th1 polarizing (IL-12,-18,-27), pro-survival (IL-7,-15, 4), and Treg inhibitory (IL-6) cytokine combinations to overcome low frequency and anergy of TAA T cells circulating in PB. Finally we genetically modified multiTAA CTLs using retroviral vectors.

Results: We were consistently able to generate multiTAA-CTLs by culturing cells in IL7, IL12, IL15 and IL27. The lines were polyclonal comprising CD4+ and CD8+ T cells, with reactivity against SSX2, Survivin and MAGEA4 as tested by intracellular cytokine staining and ELIspot $(n=5)$. The CTLs were cytolytic against autologous pepmix-pulsed or whole antigen-expressing targets in a $\mathrm{Cr}^{51}$ release assay. Importantly, this platform could also be used to generate multiTAA-CTL lines that simultaneously targeted the leukemia-TAA WT1, Prame, PR3 and Survivin $(n=5)$. Finally we demonstrated that lym-
phoma-TAA CTLs could be retrovirally transduced with a lymphoma- specific chimeric antigen receptor (CAR) targeting CD19 to further increase the breadth of specificity as well as with a chimeric IL7 cytokine receptor to allow $\mathrm{T}$ cell proliferation in the presence of the IL7 cytokine. Transduction efficiencies of $>85 \%$ were achieved without loss of native antigen specificity. Further we confirmed the function of the transgenic molecules by $\mathrm{Cr}^{51}$ release assay (CAR CD19) and proliferation assay (IL7R).

Conclusion: This study shows the successful generation of CTLs, simultaneously targeting multiple TAA antigens expressed on hematological malignancies, reducing the risk of tumor immune escape. In addition these tumor CTLs can be used as a platform for a variety of genetic modifications improving survival, migration and function.

Disclosure: No conflict of interest disclosed.

\section{V740}

\section{Comprehensive analysis of the involvement of the activating immunoreceptor NKG2D and its ligands in NK cell reactivity against leukemia}

\author{
Hilpert, J. ${ }^{1}$; Buechele, C. ${ }^{1}$; Gruenebach, F.1; Steinle, A. ${ }^{2}$; \\ Kanz, L. ${ }^{1}$; Baessler, T. ${ }^{1}$; Salih, H.R. ${ }^{1}$
}

${ }^{1}$ Eberhard Karls-University, Department of Hematology/Oncology, Tübingen, Germany, ${ }^{2}$ Goethe-University of Frankfurt, Institute of Molecular Medicine, Frankfurt, Germany

Introduction: NK cells play an important role in the immunosurveillance of leukemia. Their reactivity is guided by the principles of 'missing-self' and 'induced-self' which imply that cells with low or absent expression of MHC class I ('missing-self') and stress-induced expression of ligands of activating NK receptors ('induced-self') are recognized and eliminated by NK cells. NKG2D is a prototypic activating NK receptor and has been demonstrated to play an important role for NK reactivity against tumor cells as (i) its ligands (NKG2DL) are usually not expressed on healthy cells and (ii) sufficient expression of NKG2DL renders cancer cells susceptible to NK cell lysis despite expression of MHC class I. As the role of NKG2D and its ligands in leukemia is still controversially discussed we here comprehensively studied the expression of NKG2DL in primary leukemia cells and their capacity to activate NK reactivity.

Methods: NKG2DL expression was analyzed by FACS and quantitative PCR. To define the specific involvement of single NKG2DL we employed blocking antibodies against MICA, MICB and ULBP1-3 in analyses of NK reactivity (cytotoxicity assays, determination of CD107a as marker for granule mobilization and ELISA of IFN- $\gamma$ production).

Results: Heterogeneous expression of the NKG2DL MICA, MICB and ULBP1-3 was observed upon analysis of patient leukemia cells. Substantial (SFI $>1.5)$ surface expression of at least one NKG2DL was detected in 97 $(75.2 \%)$ of 129 investigated cases of patients with AML ( $n=54)$, ALL $(n=15)$, CLL $(\mathrm{n}=53)$ and CML ( $=7)$. In $22(22.7 \%)$ and $39(40.2 \%)$ cases, expression of two or $\geq$ three different NKG2DL was observed. Of note, in none of the patients with CML relevant NKG2DL expression was detected. Analyses of further patient samples are ongoing. Specific involvement of NKG2DNKG2DL interaction in NK reactivity against leukemia cells was demonstrated by reduction of NK reactivity upon blocking either NKG2D or its ligands in assays of allogenic NK cells and NKG2DL-expressing primary AML, ALL and CLL cells. In case that leukemia cells expressed more than one NKG2DL, additive effects were observed upon blocking two or more specific NKG2DL, which is in line with the notion that NK reactivity critically depends on NKG2DL expression levels.

Conclusion: Our data clearly demonstrate that NKG2D not only modulates NK reactivity against epithelial tumors, but also contributes to immunosurveillance of leukemia.

Disclosure: No conflict of interest disclosed. 
V741

The dual PI3 kinase/mTOR inhibitor NVP-BEZ235 acts as a suppressor of growth and function of human mast cells and basophils

\section{Blatt, K. ${ }^{1}$; Herrmann, H. ${ }^{1,2}$; Mirkina, I. ${ }^{1,2}$; Hadzijusufovic, E. ${ }^{1,3}$; Peter, B. ${ }^{1,3}$; Marth, . $^{4}$; Valenta, R. ${ }^{4}$; Valent, P. ${ }^{1,2}$}

${ }^{1}$ Medical University of Vienna, Department of Internal Medicine I, Division of Hematology \& Hemostaseology, Vienna, Austria, ${ }^{2}$ Ludwig Boltzmann Cluster Oncology, Vienna, Austria, ${ }^{3}$ University of Veterinary Medicine

Vienna, Department for Companion Animals and Horses, Clinic for Internal Medicine and Infectious Diseases, Vienna, Austria, ${ }^{4}$ Medical University of Vienna, Christian Doppler Laboratory for Allergy Research, Division of Immunopathology, Department of Pathophysiology, Center for Pathophysiology, Immunology and Infectiology, Vienna, Austria

Introduction: Growth and function of basophils (BA) and mast cells (MC) are triggered by various cytokines/ligands and signal transduction pathways. In allergic reactions, cross-linking of high affinity IgE-binding sites leads to activation and mediator secretion. In MC, the KIT-ligand SCF promotes IgE-dependent mediator secretion. In mastocytosis, KIT is mutated at codon 816 and expressed as constitutively activated target in neoplastic MC. The phosphoinositide 3-kinase (PI3 kinase) is a key signalling molecule in IgE receptor-dependent activation, and has been implicated in KIT-dependent growth and survival of normal and neoplastic cells. More recent data suggest that KIT- and IgE-receptor downstream signalling molecules represent potential therapeutic targets.

Methods: We examined the effects of the PI3 kinase/mTOR-targeting drug NVP-BEZ235 on IgE-dependent mediator release in human BA (healthy donors, $\mathrm{n}=5$; patients allergic to Bet $\mathrm{v} 1, \mathrm{n}=5$ ) and cultured cord blood progenitor-derived human MC. We also examined drug effects on growth and survival in BA and MC.

Results: NVP-BEZ235 was found to inhibit anti- IgE-induced histamine release in $\mathrm{BA}$ in all healthy individuals and $\mathrm{rBet} \mathrm{v}$ 1-induced histamine release from $\mathrm{BA}$ in all allergic patients examined $\left(\mathrm{IC}_{50} 0.5-1 \mu \mathrm{M}\right)$. NVP-BEZ235 was also found to counteract anti-IgE-induced upregulation of CD203c in BA, and to block IgE-dependent upregulation of CD63 as well as IgE-dependent mediator secretion in MC. Furthermore, NVP-BEZ235 was found to suppress IL-3-dependent development of BA and SCF-dependent development of MC from their progenitors. Finally, NVP-BEZ235 inhibited oncoprotein-dependent growth in the BA leukemia cell line KU812 and in the MC leukemia cell line HMC-1. Although growth-inhibitory effects of NVP-BEZ235 showed similar $\mathrm{IC}_{50}$ values $(0.005 \mu \mathrm{M})$ in both HMC-1 subclones, strong apoptosis-inducing effects were only seen in HMC-1.1 cells lacking KIT-D816V, but not in HMC1.2 cells expressing KIT-D816V, suggesting that the KIT-mutant introduces resistance. Most NVP-BEZ235 effects were not mimicked by the mTOR blocker RAD001, suggesting that the PI3 kinase was the primary target. Conclusions: NVP-BEZ235 inhibits IgE-dependent activation and produces growth-inhibitory effects in normal and neoplastic BA and MC. Whether these effects have clinical implications remains to be determined.

Disclosure: Katharina Blatt: No conflict of interest disclosed.

Peter Valent: Advisory Role: consulter Novartis; Financing of Scientific Research: research grant Novartis

\section{V742}

Redirecting t-cells against cancer cells by transfer of broadly tumor reactive gamma-delta-t-cell receptorsredirecting t-cells against cancer cells by transfer of broadly tumor reactive gamma-delta-t-cell receptors

\section{Marcu-Malina, V. ${ }^{1}$; Heijhuurs, S. ${ }^{1}$; Hartkamp, L. ${ }^{1}$; Kuball, J. ${ }^{1}$ UMC Utrecht, Utrecht, Netherlands}

Adoptive transfer of alpha-beta-T-lymphocytes is a promising treatment for a variety of malignancies, but often not feasible due to difficulties in generating T-cells reactive with the targeted antigen from patients. To facilitate rapid generation of cells for therapy, T-cells can be programmed with genes encoding for a tumor-specific high-affinity gamma-delta -T-cell receptor (TCR). Selective anti-tumor-reactivity and ignorance of the healthy-environment by gamma9-
delta2-T cells arises from its ability to recognize non-classical antigens, including the non-peptidic intermediates of isoprenoid biosynthesis and so called stress signals on the tumor cells. To test the ability of gamma9-delta2-TCRs to redirect alpha-beta-T cells selectively against tumor cells, the gamma9-delta2-TCR was retrovirally transduced into human alpha-beta $\mathrm{T}$ cells. Thereby, strong surfaceexpression of introduced gamma9-delta2-TCRs was observed while endogenous alpha-beta TCR chains were down-modulated. Functional analysis revealed that a gamma9-delta2-TCR efficiently reprograms both, CD4+ and CD8+ T-cells against a broad panel of cancer cells while ignoring normal cells. Moreover, tumor-specific T-cell proliferation, cytokine secretion and killing were significantly enhanced by additional application of biphosphonates. Finally, gamma9delta2-TCR transduced T cells reduced colony-formation of progenitor cells of primary acute myeloid leukemia blasts. Recognition depended primarily on the presence of phospho-antigens. Expression of NKG2D ligands and F1-ATPase contributed to the activity of gamma9-delta2-TCR transduced T-cells but were not mandatory. In summary, gamma9-delta2-TCRs are an attractive alternative to redirect alpha-beta T cells against cancer cells with both, an improved efficacy and safety profile as compared to currently used TCRs.

Disclosure: No conflict of interest disclosed.

\section{V743}

\section{Peptides incorporated into putative receptor binding} capsid domains re-direct AAV8 to the heart and AAV9 to breast tumor tissue in vivo

Michelfelder, S. ${ }^{1}$; Hunger, A. ${ }^{1}$; Varadi, K. ${ }^{2}$; Raupp, C. $^{3}$; Pahrmann, C. ${ }^{4}$; Müller, O. ${ }^{2}$; Kleinschmidt, J. ${ }^{3}$; Trepel, M. ${ }^{1}$

${ }^{1}$ University Medical Center Hamburg-Eppendorf, Hubertus-Wald Cancer Center, Department of Oncology and Hematology, Hamburg, Germany, ${ }^{2}$ University of Heidelberg, Internal Medicine III, Heidelberg, Germany, ${ }^{3}$ Deutsches Krebsforschungszentrum, Tumorvirologie, Heidelberg, Germany, ${ }^{4}$ University Heart Center Hamburg-Eppendorf, Transplant and Stem Cell Immunobiology Lab, Hamburg, Germany

Introduction: The feasibility of gene therapy in vivo will depend on the efficiency and the specificity of the applied vector system. Among the variety of AAV serotypes, AAV8 and 9 are particularly attractive due to their ability to efficiently cross vascular endothelial barriers (AAV8 and AAV9) or the bloodbrain-barrier (BBB) (AAV9). These features make them particularly suitable for systemic gene targeting approaches. Here, we identified possible receptor binding capsid regions in serotypes 8 and 9 and incorporated potential targeting peptides therein for improved transduction of cardiac and breast tumor tissue in vivo.

Methods: Potential peptide insertion sites were identified by alignment of protruding regions of the AAV-2 VP3 subunit comprising the heparin binding domain with VP sequences of serotypes 8 and 9 . A strong variability in the capsid region surrounding residues R585/R588 suggested that this domain also includes the individual attachment receptor of serotypes 8 and 9 . Based on AAV8 and 9, we generated recombinant vectors which display peptides that have been used previously by our group to re-direct the tropism of AAV2 in vivo. The peptides were inserted into capsid domains putatively involved in receptor binding. In vivo transduction of rAAV-luciferase was determined by live animal bioluminescence imaging and single organ transduction was analyzed by luminometry after systemic vector application into transgenic PymT breast cancer-bearing and wild-type mice.

Results: Insertion of the ESGLSQS peptide into AAV8 significantly increased gene delivery to the cardiac muscle while liver tropism and transduction of skeletal muscles were preserved. Insertion of ESGLSQS into AAV9 (AAV9ESG) significantly de-targeted transduction to almost all control tissues including heart and liver and restricted gene delivery to breast cancer tissue. Yet, gene expression was not entirely specific to breast tumor tissue but was also detected in the heart. However, the tumor/heart transduction ratio was higher with AAV9-ESG vectors than in AAV2-ESG vectors, while liver detargeting was more pronounced in AAV2-ESG than with AAV9-ESG vectors. Conclusions: Insertions of targeting peptides into putative receptor-binding capsid regions of AAV8 and AAV9 improve gene transduction of AAV vectors to alternative tissues in vivo. This emphasizes their potential use in therapeutic applications.

Disclosure: No conflict of interest disclosed. 
V744

\section{Tumor cell pseudoexpression of platelet derived Glucocorticoid-Induced TNF-Related (GITR) ligand impairs NK cell tumor immune surveillance}

\section{Placke, T. ${ }^{1}$; Kanz, L. ${ }^{1}$; Salih, H.R. ${ }^{1}$; Kopp, H.-G. ${ }^{1}$}

${ }^{1}$ Eberhard Karls University, Department of Hematology and Oncology, Tübingen, Germany

Introduction: NK cells as part of the innate immune system substantially contribute to cancer immune surveillance and prevent tumor progression and metastasis. NK reactivity results from an integrative response emerging upon recognition of multiple ligands for activating and inhibitory NK cell receptors. Apart from the direct interaction with their targets, NK activity is further influenced by the reciprocal interplay with other hematopoietic cells like e.g. dendritic cells. Despite clear evidence that metastatic tumor spread is dramatically reduced in thrombopenic mice which is reversed by NK cell depletion indicating that platelets impair NK anti-tumor reactivity, little is known regarding the mechanisms regulating NK cell-platelet interaction. Recently we demonstrated that NK reactivity is impaired by platelet-derived TGF- $\beta$, which is released upon interaction of platelets with tumor cells (Kopp et al., Cancer Res. 2009). Here we studied the role of GITR ligand (GITRL) in platelet-NK cell interaction.

Methods: Expression of surface molecules was investigated by FACS. NK cell functions were measured by cytotoxicity assays and ELISA.

Results: We report that the ligand for the TNFR family member GITR is upregulated on megakaryocytes during maturation resulting in substantial GITRL expression by platelets. Since we recently identified GITR as inhibitory NK receptor involved in tumor immune escape (e.g., Baltz et al., Blood 2008, Baessler et al., Cancer Res. 2009) we investigated how platelet-derived GITRL influences platelet function and NK immunosurveillance. Signaling via GITRL into platelets upon interaction with NK-expressed GITR or recombinant GITR fusionprotein did not alter platelet activation as revealed by analysis of the activation marker CD62P and TGF- $\beta$ release. Interestingly, we found that GITRL-negative tumor cells rapidly get coated by platelets, which confers a seemingly GITRL-positive phenotype. "GITRL pseudoexpression" on tumor cells caused a substantial reduction of NK cytotoxicity and cytokine production, and NK reactivity against platelet-coated tumor cells was substantially enhanced by antibody-blocking of NK cell-expressed GITR.

Conclusion: Coating of tumor cells by platelets inhibits NK reactivity, which is, at least in part, mediated by platelet-derived GITRL. Our data provide a functional basis for the previously observed finding that platelets increase metastasis by enabling tumor evasion from NK-mediated immune surveillance.

Disclosure: No conflict of interest disclosed.

\section{Freie Vorträge MDS}

\section{V745}

High-density SNP array analysis of low risk MDS according to IPSS identifies submicroscopic genomic lesions targeting genes involved in epigenetic regulation

Nowak, D. ${ }^{1}$; Ogawa, S. ${ }^{2}$; Klaumünzer, M. ${ }^{1}$; Mossner, M. ${ }^{1}$; Nolte, F. ${ }^{1}$; Nowak, V. ${ }^{1}$; Obländer, J. ${ }^{1}$; Erben, P. ${ }^{1}$; Müller, M.C. ; Sanada, M. ${ }^{2}$; Kato, M. ${ }^{2}$; Kohlmann, A. ${ }^{3}$; Haferlach, $T^{3}$; Haferlach, C. ${ }^{3}$; Koeffler, P.H. ${ }^{4}$; Hofmann, W.K. ${ }^{1}$

${ }^{1}$ Universität Heidelberg, Medizinische Fakultät Mannheim, Medizinische Klinik III, Hämatologie und Onkologie, Mannheim, Germany, ${ }^{2}$ University of Tokyo, Graduate School of Medicine, Department of Hematology and Oncology, Tokyo, Japan, ${ }^{3}$ MLL Münchner Leukämie Labor, München, Germany, ${ }^{4}$ Cedars Sinai Medical Center, UCLA School of Medicine, Division of Hematology and Oncology, Los Angeles, United States

Introduction: The development of Myelodysplastic Syndromes (MDS) is associated with the accumulation of molecular defects in hematopoietic stem cells. High-density SNP arrays are powerful tools to analyze genomic DNA to identify submicroscopic copy number alterations (CNAs), loss of heterozygosity (LOH) and uniparental disomy (UPD). In order to identify genomic lesions specific for the category of low risk MDS according to IPSS, we analyzed genomes of bone marrow cells of these patients with high-density SNP arrays.

Patients and methods: We studied 72 cases of MDS (RARS $n=22$, RCMD $\mathrm{n}=50$; age $47-89$, median 71 years). Genomic DNA was isolated from mononuclear cells from bone marrow aspirates and processed for hybridization to Affymetrix NspI 250K SNP arrays. Data analysis was carried out using the CNAG software with non-matched references.

Results: After exclusion of known copy number polymorphisms, a total of 17 deletions, 24 duplications and 12 regions of telomeric UPD were identified, which corresponded to a mean number of CNAs of $0.7 /$ sample in MDS RARS and 0.8/sample in MDS RCMD. No significant differences in patterns of CNAs were detected between these two subgroups. Common genomic lesions were identified in two samples on chromosome 20 (del20q11.21-20q13.2), in two samples on chromosome 1 (UPD 1p36.33-1p13.3) and in three samples on chromosome 4 (UPD 4q13.2-4q35.2). Interestingly, the common CNAs but also microlesions found in single samples frequently targeted genes, which are known or hypothesized to be involved in the regulation of epigenetic processes. With respect to individual genes, the common UPD regions on chromosome 4 included the TET2 gene. Deletions on chromosome 20 contained DNMT3b and ASXL1. Moreover, also CNAs in single samples affected genes, which are putatively involved in epigenetic regulation such as DNMT3b and ASXL2 on chromosome 2p23 and the histone demethylase JMJD6 on chromosome 17. Additional candidate genes such as MPL, CBL, hsa-mir-34b/c and WT1 were targeted by UPD on chromosomes 1 and 11 .

Conclusion: Our data demonstrate that high-density SNP array analysis of MDS bone marrow cells allows a detailed identification of genomic lesions, which target candidate genes known to be frequently mutated in myeloid neoplasia. Moreover, the cumulative targeting of genes hypothesized to be involved in the regulation of the epigenetic landscape such as DMNT3a/b, ASXL1/2, JMJD6 makes these interesting targets for further evaluation.

Disclosure: No conflict of interest disclosed

\section{V746}

Integrative methylation and gene expression analysis in CD34+ bone marrow cells from patients with MDS and healthy donors with Infinium 27 methylation arrays and HG-U133 plus 2 arrays

\author{
Nowak, D. ${ }^{1}$; Mossner, M. ${ }^{1} ;$ Klaumünzer, M. ${ }^{1}$; Nowak, V. ${ }^{1}$; \\ Nolte, F. ${ }^{1}$; Hofmann, W.K.
}

${ }^{1}$ Universität Heidelberg, Medizinische Fakultät Mannheim, Medizinische Klinik III, Hämatologie und Onkologie, Mannheim, Germany

Introduction: The development of myelodysplastic syndromes (MDS) is associated with the accumulation of molecular defects in hematopoietic stem cells including deregulation of epigenetic modifications such as DNA methylation. While demethylating therapeutics are achieving great successes in the treatment of MDS, the mechanisms and patterns of epigenetic deregulation in MDS are not well understood.

Methods: Infinium27 methylation arrays (Illumina) allow the genome wide analysis of $>27000 \mathrm{CpG}$ marks in the promoter regions of about $>14000$ genes and microRNAs after bisulfite conversion of genomic DNA. We tested the application of these arrays and the possibilities to integrate the methylation data with global gene expression data obtained from the same samples with HG-U133 plus 2 gene expression arrays (Affymetrix). We consecutively analyzed purified CD34+ bone marrow cells from 4 healthy donors and 4 MDS patients (MDS low risk $n=2$, MDS high risk $n=2$ ) on both types of arrays and subsequently performed data analysis to identify differentially methylated $\mathrm{CpG}$ marks and correspondingly differentially regulated genes.

Results: Genome wide methylation analysis of healthy individuals and MDS patients yielded a list of $80 \mathrm{CpGs}$, which were significantly hypermethylated in MDS patients (threshold = methylation difference $>25 \%$ ) and $93 \mathrm{CpGs,}$ which were hypomethylated in MDS patients as compared to healthy donors. The hypermethylated CpGs in MDS patients are in the promoter regions of some interesting candidate genes such as deleted in bladder cancer 1 (DBC1), cadherin 1 (CDH1), basonuclin 1 (BNC1), deleted in colorectal carcinoma (DCC), paired-like homeodomain transcription factor 2 (PITX2), Wilms tumor 1 (WT1) and cyclin-dependent kinase inhibitor $2 B(C D K N 2 B)$, which have been implicated in the pathogenesis of MDS, acute leukemia and solid tumors. 
Of the 75 hypermethylated genes, 17 genes $(23 \%)$ had a valid gene expression signature on the Affymetrix gene expression arrays. The most differentially regulated gene was $\mathrm{CDH} 1$ (methylation difference $=31 \%$ ), which was expressed in all healthy controls and low risk MDS samples but undetectable in MDS high risk samples. CDH1 has previously been indicated to be hypermethylated in MDS and prognostically relevant.

Conclusion: Combined analysis of whole genome methylation- and global gene expression data allows the identification of new candidate genes involved in the pathogenesis of MDS.

Disclosure: No conflict of interest disclosed.

\section{V747}

Comparison of differential gene expression in unselected bone marrow and purified CD34+-cells from patients with myelodysplastic syndromes

Neumann, M. ${ }^{1}$; Nolte, F. ${ }^{2}$; Benlasfer, O. ${ }^{1}$; Nowak, V. ${ }^{2}$; Schumann, C. ${ }^{2}$; Baldus, C.D. ${ }^{1}$; Thiel, E. ${ }^{1}$; Hofmann, W.-K. ${ }^{2}$

${ }^{1}$ Charité - Campus Benjamin Franklin, Department of Hematology and Oncology, Berlin, Germany, ${ }^{2}$ University Hospital Mannheim, Department of Hematology and Oncology, Mannheim, Germany

Myelodysplastic syndromes (MDS) are a heterogenous group of clonal diseases defined mainly on the level of morphology by common dysplastic features. It remains an open question, which fraction of bone marrow cells should serve as analysis material for further molecular characterization of MDS. Recently, gene expression profiling of unselected bone marrow cells failed to clearly define MDS or their subgroups on the transcriptional level. To overcome these limitations, we compared gene expression profiles of MDS patients and healthy donors in BM and individual matched $\mathrm{CD} 34^{+}$cells.

Heparinised bone marrow samples were obtained from 46 patients with MDS (low: $n=14$; intermediate-1: $n=14$; intermediate- 2 : $n=9$ and high risk: $n=9$ according to IPSS) and 14 normal individuals (NI). After separation of mononuclear cells, CD34+ cells were purified by magnetic cell separation (MACS) and high-density oligonucleotide microarray analysis (Affymetrix HG-U133 plus 2.0, data analysis by GeneSpring 4.2 and DAVID) was performed.

To define a hematopoietic stem cell (HSC) signature, we analyzed differential gene expression $\left(\mathrm{p}<0.01\right.$, Bonferroni correction) between $\mathrm{CD} 34^{+}$cells and $\mathrm{BM}$ in NI as well as in MDS. In NI the HSC signature consisted of 2205 probe sets. A high percentage (1891 probe sets, $85.8 \%$ ) of these probe sets was also included in the HSC signature in MDS. In addition, the HSC signature of MDS patients contained exclusively another 4015 probe sets indicating an altered hematopoiesis based on changes in the HSC of MDS patients. Using more stringent restrictions (at least a 3-fold change in expression), 507 probe sets were further analyzed. Pathway analysis by DAVID showed hematopoietic cell lineage and $\mathrm{T}$ cell related pathways to be mainly affected including genes like ZAP70, CD45 or LAT. Comparing the differential gene expression (3-fold change, p-value $<0.05$, present call rate higher than $75 \%$ ) between MDS and healthy individuals, the differences were more dominant in the CD34+ cells (101 probe sets) than in BM (70 probe sets). Fourteen genes were differentially expressed in both groups including genes previously reported like Early B-cell factor and Pre-B-lymphocyte 1 and 3.

These data support an involvement of immune response associated pathways in the pathogenesis of MDS. Furthermore dysbalanced gene expression was revealed in the HSC compartment in MDS compared to NI reflecting the clonal orign of MDS in the hematopoietic stem cell.

Disclosure: No conflict of interest disclosed.
V748

FISH analyses on circulating CD34+ cells in MDS: Results of the ongoing multicentric prospective diagnostic study with special focus on karyotype evolution and the correlation with conventional bone marrow diagnostics

Braulke, F.1; Schanz, J. ${ }^{1}$; Metz, M. ${ }^{2}$; Detken, S. ${ }^{3}$; Seraphin, J. ${ }^{2}$; Götze, K. ${ }^{4}$; Platzbecker, U.; Brömmendorf, T.H. ${ }^{6}$; Giagounidis, A.A.N. ${ }^{7}$; Germing, U. ${ }^{8}$; Jentsch-Ullrich, K. ${ }^{9}$; Böhme, A. ${ }^{10}$; Bug, G. ${ }^{11}$; Schafhausen, P. ${ }^{12}$; Trümper, L. ${ }^{1}$; Haase, D. ${ }^{1}$

${ }^{1}$ Universität Göttingen, Hämatologie und Onkologie, Göttingen, Germany, ${ }^{2}$ Hämato-onkologische Schwerpunktpraxis, Göttingen, Germany, ${ }^{3}$ Hämatoonkologische Schwerpunktpraxis, Northeim, Germany, ${ }^{4}$ Technische Universität München, Hämatologie / Onkologie, München, Germany, 5 Universität Dresden, Hämatologie / Onkologie, Dresden, Germany, ${ }^{6}$ Universität Aachen, Hämatologie / Onkologie, Aachen, Germany, ${ }^{7}$ Klinikum Duisburg, Hämatologie / Onkologie, Duisburg, Germany, ${ }^{8}$ Universität Düsseldorf, Hämatologie / Onkologie, Düsseldorf, Germany, ${ }^{9}$ Hämato-onkologische Schwerpunktpraxis, Magdeburg, Germany,

${ }^{10}$ Hämato-onkologische Schwerpunktpraxis, Frankfurt, Germany,

${ }^{11}$ Universitätsklinik Frankfurt, Hämatologie / Onkologie, Frankfurt, Germany, ${ }^{12}$ Universität Hamburg Eppendorf, Hämatologie / Onkologie, Hamburg, Germany

Purpose: In myelodysplastic syndromes (MDS) chromosomal aberrations play a major role in pathogenesis, prognosis, diagnostics and treatment allocations. Chromosomal anomalies in MDS patients (pts) are provable in bone marrow (bm) cells by chromosome banding analyses as well as in enriched circulating CD34+ cells from peripheral blood (pb) by Fluorescence in situ hybridization (FISH).

Methods: In 10/2008 we started a German multicentric prospective diagnostic study to follow chromosomal anomalies by FISH in pts with suspected or cytomorphologically assured MDS: they are initially screened by FISH analyses on immunomagnetically enriched circulating CD34+ cells from pb using a "superpanel" (D7/CEP7, EGR1, CEP8, CEP XY, D20, p53, IGH/BCL2, TEL/ AML1, RB1, MLL, 1p36/1q25, CSF1R) and followed by FISH analyses every 2 months within the 1 . year and every 3 months in the 2 . year using a "standardpanel" (EGR1, D7/CEP7, CEP8, p53, D20, CEP X/Y, TEL/AML1). The "superpanel" will be repeated every 12 months and in every case of suspected progression.

Aims: Chromosomal anomalies in MDS pts can be diagnosed and followed by frequent sequential FISH analyses, karyotype evolution (KE) can be detected, and we expect more information about rare abnormalities. All results from $\mathrm{pb}$ will be correlated with FISH analyses from bm, if available, and compared to the results from banding analyses. $\mathrm{Pb}$ counts, transfusion-dependence and therapies are documented in a database.

Results: After 16 months of study time 154 pts from 12 German centers are included. The cohort is representative for MDS according to sex, age, subtypes and therapy regimens (101 BSC, 25 5-Aza, 10 lenalidomide, 5 AZA-LE, 5 valproic acid, 3 allogeneic SCT, 5 other). In 58\% (89/154) of pts. chromosomal aberrations were provable by FISH. There were 48 pts with del $(5 q), 30$ pts with $\operatorname{del}(7 q) /-7,13$ with loss of TP53, 10 with $\operatorname{del}(20 q)$, and 11 pts with $\operatorname{del}(12 \mathrm{p})$. As yet, a KE could be diagnosed from pb in 15 pts $(14 \%)$ with at least 2 timepoints of analysis available.

Conclusions: FISH analyses of enriched circulating CD34+ cells is a feasable method to detect chromosomal aberrations in MDS pts from pb. It allows a very frequent monitoring and provides the possibility to observe KE prospectively for the first time step by step and to document the appearance and disappearance of anomalies under therapy. We correlate the results from pb FISH with bm analyses and demonstrate interesting and new findings in MDS cytogenetics.

Disclosure: No conflict of interest disclosed. 
V749

\section{Loss of the Y Chromosome in MDS: Clonal abnormality or age-related accident?}

Ganster, C. ${ }^{1}$; Schanz, J. ${ }^{1}$; Braulke, F. ${ }^{1}$; Shirneshan, K. ${ }^{1}$; Solé, F.2; Hildebrandt, B. ${ }^{3}$; Slovak, M.L. ${ }^{4}$; Ohyashiki, K. ${ }^{5}$; Steidl, C. ${ }^{1}$; Fonatsch, C. ${ }^{6}$; Pfeilstöcker, M. ${ }^{7}$; Nösslinger, T.7; Valent, P. ${ }^{8}$; Giagounidis, A. ${ }^{9}$; Aul, C. ${ }^{9}$; Lübbert, M. ${ }^{10}$; Stauder, R. ${ }^{11}$; Krieger, O. ${ }^{12}$; Le Beau, M. ${ }^{13}$; Bennett, J. ${ }^{14}$; Greenberg, P. ${ }^{15}$; Germing, U. ${ }^{16}$; Haase, D. ${ }^{1}$, Deutsch-ÖsterreichischSchweizerische MDS-Studiengruppe (DACH), International Cytogenetics Work Group der MDS Foundation (ICWG), International MDS Risk Analysis Workshop (IMRAW)

${ }^{1} \mathrm{Hämatologie}$ und Onkologie, Universitätsmedizin Göttingen, Göttingen, Germany, ${ }^{2}$ Laboratori de Citogenètica i Biologia Molecular, Barcelona, Spain, ${ }^{3}$ Institut für Humangenetik, Heinrich-Heine-Universität Düsseldorf, Düsseldorf, Germany, ${ }^{4}$ Division of Pathology, City of Hope, Duarte, United States, ${ }^{5}$ Faculty of Medicine, Tokyo Medical University, Tokyo, Japan, ${ }^{6}$ Department für Medizinische Genetik, Medizinische Universität Wien, Wien, Austria, ${ }^{7}$ Medizinisches Departement, Hanusch Hospital, Wien, Austria, ${ }^{8}$ Klinische Abteilung für Hämatologie und Hämostaseologie, Medizinische Universität Wien, Wien, Austria, ${ }^{9}$ Medizinische Klinik II, St. Johannes Hospital, Duisburg, Germany, ${ }^{10} \mathrm{Hämatologie} \mathrm{und} \mathrm{Onkologie,}$ Universitätsklinikum Freiburg, Freiburg, Germany, ${ }^{11}$ Hämatologie und Onkologie, Medizinische Universität Innsbruck, Innsbruck, Austria, ${ }^{12} \mathrm{Hämatologie} \mathrm{und} \mathrm{Onkologie,} \mathrm{Krankenhaus} \mathrm{der} \mathrm{Elisabethinen,} \mathrm{Linz,}$ Austria, ${ }^{13}$ Section of Hematology/Oncology, Cancer Research Center, Chicago, United States, ${ }^{14}$ Department of Pathology, Hematopathology Division, Wilmot Cancer Center, Rochester, United States, ${ }^{15}$ Department of Hematology, Stanford University School of Medicine, Palo Alto, United States, ${ }^{16} \mathrm{~K}$ linik für Hämatologie, Onkologie und Klinische Immunologie, Heinrich-Heine-Universität Düsseldorf, Düsseldorf, Germany

Introduction: Loss of the $\mathrm{Y}$ chromosome is frequently observed in males as an age-related phenomenon (UKCCG, 1992), but it also has been reported to be associated with hematopoietic diseases (Wiktor et al., 2000). Differentiation between age- and MDS-associated Y-loss might be of value in determining clonality and predicting prognosis and treatment outcome. The aim of this study was the characterization of MDS patients with -Y, concentrating on the evaluation of Y-loss as age- or MDS-associated phenomenon.

Patients and methods: We identified 101 patients with -Y in our multicentre, international DACH-, ICWG- and IMRAW-database and analyzed them according to age, clone size, and additional chromosomal aberrations. To monitor the natural course of the cytogenetic anomalies, we only included patients with primary, untreated MDS. The prognostic relevance of the aberrations was assessed using univariate and multivariate models.

Results: Loss of the $Y$ chromosome was detected in 3.5\% ( $n=101)$ of patients of the multicentre MDS-database. Loss of Y as a single aberration was seen in $65.3 \%(n=66)$ of these patients, in $14.9 \%(n=15)$ it was observed together with one further aberration and in $19.8 \%(\mathrm{n}=20)$ within a complex aberrant karyotype. Overall survival of patients with $-\mathrm{Y}$ as a sole abnormality was significantly better compared to patients with a normal karyotype (60.8 vs. 47.4 months; hazard ratio $=0.50, \mathrm{p}<0.01)$. As a single aberration, it was significantly more frequent in older ( $>60$ years) than in younger patients $(4.0 \% \mathrm{vs.}$ $1.9 \%, \mathrm{p}<0.01)$. In contrast to other abnormalities, patients showing $-\mathrm{Y}$ were older at time of first diagnosis ( 66.7 vs. 71.5 years, $\mathrm{p}<0.01)$. There were no differences in clone size between patients with $-Y$ and patients with other aberrations. We observed MDS patients with -Y occurring during karyotype evolution and propose that $-\mathrm{Y}$ is MDS-associated in this group of patients. Conclusions: The clearly better prognosis of MDS patients with $-\mathrm{Y}$ as the single aberration compared to MDS patients with normal karyotype as well as cases with -Y occurring during karyotype evolution suggests a clonal nature of this chromosomal anomaly at least in a portion of patients with MDS. The hypotheses established by retrospectively analyzing our data will be tested in an experimental study by analyzing CD3+ cells not belonging to the MDS clone and clonal CD34+ cells of MDS patients with -Y.

Disclosure: No conflict of interest disclosed.
V750

\section{Results from the Austrian Project analysing retrospectively the use of Lenalidomide in patients with myelodysplatic syndroms (REALM)}

\author{
Aschauer, G. ${ }^{1}$; Greil, R. ${ }^{2}$; Linkesch, W. ${ }^{3}$; Pfeilstöcker, M.4. \\ Stauder, R. ${ }^{5}$; Thaler, J. ${ }^{6}$; Fiegl, M. ${ }^{7}$; Fridrik, M. ${ }^{8}$; Girschikofsky, \\ M. ${ }^{9} ;$ Keil, F. ${ }^{10}$; Petzer, A.L. ${ }^{1}$
}

${ }^{1} \mathrm{KH}$ der Bamherzigen Schwestern Linz, Hämatologie und Onkologie, Linz, Austria, ${ }^{2}$ Universität Salzburg, Innere Medizin III, Salzburg, Austria, ${ }^{3}$ Universität Graz, Interne I, Hämatologie und Onkologie, Graz, Austria, ${ }^{4}$ Hanusch Krankenhaus, 3. Med. Abteilung f. Hämatologie und Onkologie, Wien, Austria, ${ }^{5} \mathrm{Med}$. Universität Innsbruck, Hämatologie und Onkologie, Innsbruck, Austria, ${ }^{6}$ Klinikum der Kreuzschwestern Wels, 4. Innere Abteilung, Hämatologie und Onkologie, Wels, Austria, ${ }^{7}$ LKH Natters, Internistische Onkologie, Nattes, Austria, ${ }^{8} \mathrm{AKH}$ Linz, Abteilung f. Hämatologie und Onkologie, Linz, Austria, ${ }^{9} \mathrm{KH}$ der Elisabethinen Linz, Hämatologie und Onkologie, Linz, Austria, ${ }^{10} \mathrm{KH}$ Leoben, Abteilung $\mathrm{f}$. Hämatologie und Onkologie, Leoben, Austria

Introduction: Several studies have demonstrated that IMIDs are effective in patients with myelodysplastic syndroms (MDS), especially in patients harbouring a deletion $5 \mathrm{q}$. This has led to an approval of the IMID lenalidomide for patients with MDS in the United States of America but not in Europe. Nevertheless, based on the impressive results of lenalidomide in various studies, it is already used by many hematologists in Europe including Austria.

Methods: We set up a registry concerning usage of this new drug in a "realworld" treatment setting. In this retrospective analysis 50 patients were identified in 16 different Austrian hospitals. This represents approximately $45 \%$ of all MDS patients that were treated with lenalidomide in Austria between April 2006 and March 2009. Of these 50 patients, 33 were females and 17 males. The median age of this cohort was 74 years. In total, 23 patients had a $5 \mathrm{q}-$ syndrom, 5 patients had MDS with isolated 5q deletion with $>5 \%$ blasts in the bone marrow including 1 patient with AML, 22 patients had other MDS entities according to the WHO classification.

Results: Overall, all patients received a median of 4.5 cycles of lenalidomide. In contrast, the cohort with a $5 \mathrm{q}$ - syndrom received a median of 10 cycles. Prescribed daily doses of lenalidomide for 21 days in a 4 weeks cycle were: $10 \mathrm{mg}$ (27 patients), $5 \mathrm{mg}$ (20 patients), $2.5 \mathrm{mg}$ (1 patient), $15 \mathrm{mg}$ (1 patient) and $20 \mathrm{mg}$ (1 patient), respectively. Dose interruptions due to treatment related adverse events were performed in 15 patients. Out of 46 patients that were evaluable according to the International Prognostic Scoring System (IPSS), 16 patients were at low, 8 patients intermediate-1, 16 patients intermediate- 2 and 3 patients at high risk. The ECOG performance status was " 0 " in $30 \%$, " 1 " in $43 \%$, "2" in $21 \%$ and " 3 " in $6 \%$ of the patients. In total, 36 patients were evaluable regarding transfusion independence. In this respect, $64 \%$ of patients responded to lenalidomide. A comparable amount responded in the $5 \mathrm{q}-\mathrm{syn}-$ drom group (66\%). Transformation to AML in the 5q- subgroup occurred in $6.9 \%$ and $25 \%$ in all patients.

Conlusions: Lenalidomide used in MDS patients in Austria outside a clinical study showed a similar efficacy as reported in studies with lager patient cohorts. The rate of transformations to secondary AML during or after the treatment with lenalidomide was not higher as can be expected without treatment with lenalidomide.

Disclosure: Gregor Aschauer: Financing of Scientific Research: unrestricted research grant from Celgene

Andreas Petzer: Financing of Scientific Research: unrestricted research grant from Celgene. 


\section{Freie Vorträge \\ Kolorektale Karzinome}

\section{V751}

Distinct classes of tumor initiating cells form tumor and metastases in human colon cancer

Dieter, S.M. ${ }^{1}$; Ball, C.R. ${ }^{1}$; Hoffmann, C.M. ${ }^{1}$; Nowrouzi, A. ${ }^{1}$; Brand, K. ${ }^{2}$; Schmidt, M. ${ }^{1}$; Koch, M. ${ }^{3}$; Weitz, J. ${ }^{3}$; von Kalle, C. ${ }^{1}$; Glimm, $H^{1}{ }^{1}$

${ }^{1}$ Nationales Centrum für Tumorerkrankungen (NCT), Deutsches Krebsforschungszentrum (DKFZ), Translationale Onkologie, Heidelberg, Germany, ${ }^{2}$ Pathologisches Institut, Universitätsklinikum Heidelberg, Heidelberg, Germany, ${ }^{3}$ Universitätsklinik Heidelberg, Allgemein-, Viszeral- und Transplantationschirurgie, Heidelberg, Germany

Human colon cancer harbors a small subfraction of tumor initiating cells (TIC) that are exclusively capable of tumor formation in immunodeficient mice. Often termed cancer stem cells, TIC are assumed to be a homogeneous stem-cell-like population that drives tumor maintenance and metastasis formation. Using a molecular tracking strategy, we found an unexpected cellular heterogeneity within the TIC compartment in vivo.

TIC derived from different primary human colon tumors or metastases were enriched in tumor spheres. In vivo self-renewal and metastatic capacity of single TIC were analyzed in serially transplanted tumors and metastases by highly sensitive tracking of individual lentivirally marked clones. Marked primary tumor cells were serially transplanted under the kidney capsule of immunodeficient NOD.Cg-Prkdc ${ }^{\text {scid }} / 12^{\text {rgtm } 1 \mathrm{Wj} /} / \mathrm{SzJ}$ (IL2RG ${ }^{-1}$ ) mice. Clonal contribution of TIC individually marked by unique lentiviral integration sites was detected by linear amplification-mediated PCR (LAM-PCR) followed by next generation high throughput sequencing.

Large-scale insertion site analysis of lentiviral marking allowed to distinguish three types of TIC contributing to tumor formation upon serial transplantation into IL2RG ${ }^{-/}$mice. Extensively self-renewing long-term tumor initiating cells (LT-TIC) were found to maintain tumor formation in serial transplants. A larger number of tumor transient amplifying cells (T-TAC) with limited or no self-renewal capacity contributed to tumor formation exclusively in primary mice. Furthermore, a subpopulation of rare dormant TIC was active only in secondary or tertiary mice. Importantly, metastasis formation was predominantly driven by LT-TIC.

Whereas only single TIC clones were present in the peripheral blood, multiple TIC clones homed to the bone marrow. Low in absolute numbers, tumor cells in the bone marrow revealed tumorigenic potential upon transplantation, indicating that the bone marrow can serve as a reservoir for metastatic TIC.

Our data demonstrate that tumor initiation, self-renewal as well as metastasis forming potential are limited to particular classes of TIC that are hierarchically organized. These cell classes can be highly enriched and clonally tracked in vivo. Our results thereby identify LT-TIC as the ideal target to improve colon cancer treatment towards the eradication of self-renewing tumorigenic and metastatic colon cancer cells.

Disclosure: No conflict of interest disclosed.

\section{V752}

Induction of apoptosis by the p14 ${ }^{\mathrm{ARF}}$ tumour suppressor is fully independent of p53 and involves nonmitochondrial death pathways in colorectal cancer

Overkamp, T.1,2; Müer, A. ${ }^{1,2}$; Gillissen, B. ${ }^{1,2}$; Wendt, J. ${ }^{1,2}$; Dörken, B. ${ }^{3}$; Daniel, P. ${ }^{1,2,3}$; Hemmati, P. ${ }^{1,2,3}$

${ }^{1}$ Charité - Universitätsmedizin Berlin, Experimental and Clinical Research Center, Berlin, Germany, ${ }^{2}$ Max-Delbrück-Center for Molecular Medicine, Clinical and Molecular Oncology, Berlin, Germany, ${ }^{3}$ Charité -

Universitätsmedizin Berlin, Medizinische Klinik m.S. Hämatologie und Onkologie, Berlin, Germany

Introduction: $\mathrm{P} 14^{\mathrm{ARF}}$, the alternative reading frame (ARF) product of the INK4A gene locus, plays a central role in the regulation of cell death, cell cycle arrest, and senescence following oncogene activation or DNA damage in the majority of human neoplasms including colorectal cancer (CRC). In p53- proficient CRC cells expression of p14ARF triggers the up-regulation of the pro-apoptotic BH3-only Bcl-2 family member PUMA ( $p 53$ upregulated mediator of apoptosis) and its effector Bax. This, in turn, results in the activation of mitochondria, caspase cleavage, and subsequent cell death. Nonetheless, p14ARF triggers p53-independent apoptosis, which may be executed by nonmitochondrial cell death pathways.

Methods and results: To further explore the role of p53 and its homologs p63 or p73 and to elucidate alternate cell death pathways in mediating apoptosis upon activation of the p14ARF tumor suppressor pathway in CRC, a dominant negative $\mathrm{p} 53$ protein $(\triangle \mathrm{Np} 53)$ was expressed in the human $\mathrm{CRC}$ cell lines HCT116-p53+/+ or HCT116-p53-/-. Following expression of exogenous p14ARF, BH3-only proteins, e.g. Puma, Noxa, or Bim, and BH123 proteins, i.e. Bax and Bak, were found up-regulated at both the mRNA and protein level irrespective of the presence or absence of a functional p53, p63, or p73. In addition to triggering the mitochondrial death machinery, as indicated by a breakdown of the mitochondrial membrane potential, release of cytochrome c, and subsequent activation of caspases, expression of p14ARF engaged the endoplasmatic reticulum (ER) stress pathway. This could be blocked by ER-targeted over-expression of anti-apoptotic Bcl-2 family proteins or the specific knock-down of pro-apoptotic Bcl-2 homologs by siRNA. However and in contrast to ER stressor drugs tunicamycin or thapsigargin, expression of $\mathrm{p} 14^{\mathrm{ARF}}$ did not up-regulate the endoplasmic BiP/Grp78 chaperone.

Conclusions: Our data delineate that in a model of human CRC apoptosis induction by the p14ARF tumor suppressor is p53-independent. Furthermore, our data show that p14ARF triggers an imbalance in the homeostasis between pro- and anti-apoptotic Bcl-2 family proteins at the ER irrespective of the presence of absence of a functional p53 signalling axis or pro-apoptotic Bax. This, in turn, may have profound consequences with regards to the development of novel treatment strategies for patients with CRC harbouring p53 and/or Bax mutations.

Disclosure: No conflict of interest disclosed.

\section{V753}

\section{Single nucleotide polymorphisms in the hypoxia-inducible} factor 1 gene and colorectal cancer risk

\section{Knechtel, G. ${ }^{1}$; Szkandera, J. ${ }^{1}$; Stotz, M. ${ }^{1}$; Hofmann, G. ${ }^{1}$;} Langsenlehner, U. ${ }^{2}$; Krippl, P. ${ }^{3}$; Samonigg, $H^{1}{ }^{1}$; Renner, W. ${ }^{4}$; Dehchamani, D. ${ }^{5}$; Gerger, A. ${ }^{1}$

${ }^{1}$ Medical University Graz, Department of Internal Medicine, Division of Oncology, Graz, Austria, ${ }^{2}$ Internal Outpatient Department, Steiermaerkische Gebietskrankenkasse, Graz, Austria, ${ }^{3}$ Department of Internal Medicine, Regional Hospital of Fürstenfeld, Fürstenfeld, Austria, ${ }^{4}$ Medical University Graz, Clinical Institute of Medical and Laboratory Diagnostics, Graz, Austria, ${ }^{5}$ Medical University Graz, Department of Dentistry and Maxillofacial Surgery, Division of Oral and CranioMaxillofacial Surgery, Graz, Austria

With an incidence of about 300.000 new cases colorectal cancer (CRC) is the second leading cause of cancer-related death in Europe and the United States. Environmental and genetic factors influence CRC risk. Hypoxia-inducible factor-1 (HIF-1), a heterodimeric protein composed of two subunits, HIF-1 alpha and HIF-1 beta, plays a critical role in oxygen homeostasis and is involved in angiogenesis and cell proliferation. The gene for the HIF-1 alpha subunit (HIF1A) carries two common missense mutations - P582S (rs11549465) and A588T (rs11549467) - which both have been related to increased transactivation capacity of HIF1A. In our case-control study we investigated the association between these polymorphisms and CRC risk. We investigated 381 patients with histologically confirmed CRC and 2156 control subjects. HIF1A genotypes were determined by exonuclease (TaqMan) assays. HIF1A P582S and A588T genotype frequencies were not significantly different between patients (PP 76.4\%, PS/SS 20.2\%; AA 93.4\%; AT/TT 2.9\%) and control subjects (PP 82.2\%, PP/PS 17.8\%, p=0.147; AA 96.5\%, AT/TT 3.5\%, p=0.608). In a multivariate logistic regression analysis including age and sex neither the HIF1A 582S allele (Odds ratio: 1.204; 95\% confidence interval 0.911 - 1.592; $\mathrm{p}=0.193$ ) nor the $588 \mathrm{~T}$ allele was significantly associated with CRC (Odds ratio: $0.851 ; 95 \%$ confidence interval $0.444-1.631 ; \mathrm{p}=0.626$ ). However, in an exploratory analysis, the HIF1A 588T allele was associated with tumor localization $(\mathrm{p}=0.016)$ and tumor size $(\mathrm{p}=0.003)$. We conclude that functional 
polymorphisms in the HIF1A gene do not modify CRC risk but maybe associated with clinic-pathological features of the disease.

Disclosure: No conflict of interest disclosed.

\section{V754 \\ Association of hypoxia inducible factor 1-alpha gene polymorphisms and colorectal cancer prognosis}

Szkandera, J. ${ }^{1} ;$ Knechtel, G. ${ }^{1}$; Stotz, M. ${ }^{1}$; Hofmann, G. ${ }^{1}$; Langsenlehner, U.2; Krippl, P. . ; Langsenlehner, T.4; Dehchamani, D. ${ }^{5}$; Samonigg, $H^{1}{ }^{1}$; Renner, W. ${ }^{6}$; Gerger, A. ${ }^{1}$ ${ }^{1}$ Universitätsklinik für Innere Medizin, Abteilung für Onkologie, Graz, Austria, ${ }^{2}$ Steiermärkische Gebietskrankenkasse, Ambulanz für Innere Medizin, Graz, Austria, ${ }^{3}$ Landeskrankenhaus Fürstenfeld, Abteilung für Innere Medizin, Fürstenfeld, Austria, ${ }^{4}$ Universitätsklinik für Strahlentherapie-Radioonkologie, Graz, Austria, ${ }^{5}$ Universitätsklinik für Zahn-, Mund- und Kieferheilkunde, Abteilung für Mund-, Kiefer- und Gesichtschirurgie, Graz, Austria, ${ }^{6}$ Universitätsklinik Graz, Klinisches Institut für Medizinische und Chemische Labordiagnostik, Graz, Austria

Introduction: Hypoxia inducible factor-1 (HIF-1) is the key regulator of cellular responses to hypoxia and plays a central role in tumour growth. Recently, two single nucleotide polymorphisms (SNP) in the HIF-1alpha gene, C1772T and G1790A, were shown to cause significantly higher transcriptional activity than wild-type. This study aimed to investigate the effect of these SNPs on the prognosis of colorectal cancer (CRC).

Methods: DNA from 336 CRC patients was genotyped. Genotypes of each polymorphism were tested for association with disease-free-survival (DFS) using univariate and multivariate Cox-regression analysis.

Results: Genotype frequencies were: CC $75.6 \%$, CT $18.8 \%$ and TT $1.8 \%$ for HIF-1alpha C1772T and GG 93.2\%, GA 2.7\% and AA 0\% for G1790A. A statistically significant association between DFS and clinic-pathological features was observed. However, no association was found between HIF-1alpha C1772T ( $\mathrm{p}=0.44, \mathrm{RR}=1.19,95 \% \mathrm{CI}=0.77$ to 1.83$)$ and $\mathrm{G} 1790 \mathrm{~A}(\mathrm{p}=0.89$, $\mathrm{RR}=0.92,95 \% \mathrm{CI}=0.29$ to 2.90 ) polymorphisms and DFS in univariate and multivariate Cox-regression analysis (RR: risk ratio of recurrence, CI: confidence interval)

Conclusion: These results suggest that HIF-1alpha C1772T and G1790A polymorphisms are not involved in the progression or metastasis of CRC.

Disclosure: No conflict of interest disclosed.

\section{V755}

Analysis of the randomized trial of the German AIO CRC study group: Cetuximab plus XELIRI versus cetuximab plus XELOX as first-line treatment for patients with metastatic colorectal cancer (mCRC)

Stintzing, S. ${ }^{1}$; Jung, A. ${ }^{2}$; Vehling-Kaiser, U. ${ }^{3}$; Stauch, M. ${ }^{4}$; Hass, $H^{5}{ }^{5}$, Dietzfelbinger, $H_{.6}{ }^{6}$; Fischer von Weikersthal, $L^{7}$. Moosmann, N. ${ }^{1}$; Kirchner, T. $^{2}$; Heinemann, V. $^{1}$

${ }^{1}$ Klinikum Grosshadern, Ludwig-Maximilians-Universität, Hämatolgie und Onkologie, München, Germany, ${ }^{2}$ Pathologisches Institut der LMU, München, Germany, ${ }^{3}$ Onkologische Praxis, Landshut, Germany,

${ }^{4}$ Onkologie Praxis, Kronach, Germany, ${ }^{5}$ Marienhospital, Stuttgart, Germany, ${ }^{6}$ Onkologie Praxis, Herrsching, Germany, ${ }^{7}$ Klinikum St. Marien, Amberg, Germany

Introduction: Cetuximab combined with 5-fluorouracil/folinic acid plus irinotecan or oxaliplatin has shown activity in the treatment of mCRC. This randomized phase II trial investigated the efficacy and safety of the epidermal growth factor receptor antibody cetuximab combined with the oral fluoropyrimidine capecitabine plus irinotecan (XELIRI) or oxaliplatin (XELOX) in the first-line treatment of $\mathrm{mCRC}$

Methods: A total of $185 \mathrm{mCRC}$ patients were randomized to cetuximab $\left(400 \mathrm{mg} / \mathrm{m}^{2}\right.$ day 1 , followed by $250 \mathrm{mg} / \mathrm{m}^{2}$ weekly) plus XELIRI (irinotecan $200 \mathrm{mg} / \mathrm{m}^{2}$, day 1 ; capecitabine $800 \mathrm{mg} / \mathrm{m}^{2}$ twice daily days $1-14$, every 3 weeks; $20 \%$ dose reduction of both agents for patients older than 65 years) or cetuximab plus XELOX (oxaliplatin $130 \mathrm{mg} / \mathrm{m}^{2}$ day 1 ; capecitabine $1000 \mathrm{mg} /$ $\mathrm{m}^{2}$ twice daily days $1-14$, every three weeks). The primary study endpoint was objective response rate (ORR). KRAS mutation status (wild-type or mutant) was determined on codons 12/13/61/146 using a mutation-specific quantitative PCR-based assay.

Results: In the intention-to-treat patient population ( $n=177)$, ORR was $47.2 \%$ (95\% CI: $36.5-58.1)$ versus $47.7 \%$ (95\% CI: 37-58.7) and the disease control rate $74.2 \%$ versus $77.3 \%$ for cetuximab plus XELIRI versus cetuximab plus XELOX. Time to progression and overall survival were 6.4 and 21.1 months for cetuximab and XELIRI compared to 8.2 and 25.5 months for cetuximab and XELOX. Determination of KRAS status was possible in $78.5 \%$ of patients $(n=139) .61 .2 \%$ of those patients showed a KRAS wild-type, and $38.8 \%$ a mutant KRAS status. No differences regarding ORR, DCR, OS or PFS could be observed in KRAS wild-type compared to KRAS mutated patients. Both study treatments had manageable tolerability profiles and were safe. The most common grade $3 / 4$ toxicities in the cetuximab plus XELIRI arm versus cetuximab plus XELOX arm were diarrhea (15.7\% versus $19.3 \%)$, cetuximabinduced exanthema ( $13.5 \%$ versus $20.5 \%)$, and sensory neurotoxicity $(1.1 \%$ versus $14.8 \%$ )

Conclusion: This randomized trial demonstrates the efficacy and tolerability of cetuximab combined with XELIRI or XELOX for the first-line treatment of patients with mCRC, while KRAS mutation status was not predictive of treatment outcome.

Disclosure: No conflict of interest disclosed.

\section{V756}

Secondary resection of liver metastasis in non-selected patients with FUFIRI or mIROX for first-line treatment in metastatic colorectal cancer (mCRC): A post-hoc analysis of tumor response and overall survival in the FIRE-trial

\section{Gießen, C. ${ }^{1}$; Schalhorn, A. ${ }^{1}$; Stintzing, S. ${ }^{1}$; Heinemann, V. ${ }^{1}$}

${ }^{1}$ Klinikum der Universität München - Großhadern, Medizinische Klinik III, München, Germany

Background: Chemotherapy in metastatic colorectal cancer (mCRC) can downsize colorectal liver metastasis for curative secondary resection. We evaluated downsizing and survival of FUFIRI and mIROX treatment in nonselected patients in this setting.

Patients and methods: Data from a phase III, randomized, open-label multicenter study with 479 patients treated with standard treatment with FUFIRI (irinotecan $80 \mathrm{mg} / \mathrm{m}^{2}, 5-\mathrm{FU} 2000 \mathrm{mg} / \mathrm{m}^{2}$, folinic acid $500 \mathrm{mg} / \mathrm{m}^{2}$ weekly) or mIROX (irinotecan $80 \mathrm{mg} / \mathrm{m}^{2}$ plus oxaliplatin $85 \mathrm{mg} / \mathrm{m}^{2}$ weekly) applied on days 1, 15, and 29 of a 7 -week cycle were evaluated. Assessment of the largest hepatic lesion was performed at the time of randomization and before surgical resection.

Results: Secondary liver resection was performed in 23 patients of the FUFIRI arm $(9.7 \%)$ and 15 patients of the mIROX arm $(6.2 \%)(\mathrm{p}=0.179)$. Total resection rate of the study was $7.9 \%$ (38/479). R0-resection could be realized in 29 cases ( 18 vs. 11 , FUFIRI vs. mIROX, $\mathrm{p}=0.169)$. Resection rates in the subgroup of liver-only patients $(248 / 479,51.8 \%)$ were $20.2 \%$ (FUFIRI) and $11.2 \%$ (mIROX) $(\mathrm{p}=0.050)$. R0-resections $15.8 \%$ and $8.2 \%,(\mathrm{p}=0.064)$. In both groups highly significant downsizing of liver metastasis with $33.3 \%$ reduction of length, $35.0 \%$ reduction in width and $57.6 \%$ reduction of surface of the largest lesion could be achieved $(\mathrm{p}<0.001)$. Identical objective response rate (ORR) of $41 \%$ could be induced in both regimens but disease control rate $(\mathrm{DCR}=$ ORR plus SD) was significantly greater in the FUFIRI group $(81 \%$ vs. $69 \%$, $\mathrm{p}=0.001$ ). These findings were consistent with results of the liveronly subgroup ( $87 \%$ vs. $67 \%, p<0.001)$. Median overall survival (OS) of all 38 resected patients was 45.8 months. One-year-survival and 5-year-survival was $97.4 \%$ and $37.1 \%$, respectively.

Conclusion: Both FUFIRI and mIROX treatment showed highly significant reduction of colorectal liver metastasis. There was a trend towards a higher resection rate and R0-resections in the liver-only subgroup for FUFIRItreatment. This trend may be explained by higher disease control rates in the FUFIRI patients. Patients who underwent hepatic resection showed favorable long-term survival with median OS 45.8 months. and 5-year-survival of $37.1 \%$.

Disclosure: No conflict of interest disclosed. 
Freie Vorträge

Chronische Myeloproliferative Neoplasien

\section{V757}

\section{Contribution of a highly sensitive JAK2 ${ }^{\mathrm{V} 617 \mathrm{~F}}$ quantitative assay to the determination of normal values}

\section{Martinaud, C. ${ }^{1}$; Maroc, N. ${ }^{2}$; Hermitte, F. ${ }^{2}$; Mozziconacci, M.-J. ${ }^{3}$ ${ }^{1}$ Hôpital d'Instruction des Armées, HIA Sainte-Anne, Toulon, France, ${ }^{2}$ Ipsogen, Marseille, France, ${ }^{3}$ Institut Paoli-Calmettes, Biopathology Department, Marseille, France}

Background: The JAK2 ${ }^{\mathrm{V} 617 \mathrm{~F}}$ mutation has been described in 2005 in patients with Philadelphia negative myeloproliferative neoplasms (MPN), and is detected in $95 \%$ of patients with PV, and approximately $50 \%$ of patients with ET or PMF. Its assessment is now part of the diagnostic workup for MPN established by the 2008 WHO guidelines, and positivity for JAK2 ${ }^{\mathrm{V} 617 \mathrm{~F}}$ mutation is a major criterion for diagnosis of PV and ET. These, combined with the constant improvement in sensitivity of detection techniques, reinforce the need for a proper definition of a positivity cut-off, which would distinguish true positive cases from background levels of JAK2 ${ }^{\mathrm{V} 617 \mathrm{~F}}$. This study aimed at establishing the background level of JAK2 ${ }^{\mathrm{V} 617 \mathrm{~F}}$ mutation in healthy individuals (HI).

Methods: 200 randomly selected HI were assessed. Exclusion criteria were: age $<18 \mathrm{y}$, medical history of deep thrombosis or blood disease, haemoglobin $>185 \mathrm{~g} / \mathrm{L}(\mathrm{M})$ or $>165 \mathrm{~g} / \mathrm{L}(\mathrm{F})$ or hematocrit $>52 \%(\mathrm{M})$ or $>48 \%(\mathrm{~F})$, WBC $>12 \mathrm{G} / \mathrm{L}$, platelet count $>400 \mathrm{G} / \mathrm{L}$. Genomic DNA was extracted from whole blood. The JAK2 ${ }^{\mathrm{V} 617 \mathrm{~F}}$ mutation was quantified using a highly sensitive allele specific RQ-PCR assay (JAK2 MutaQuant ${ }^{\mathrm{TM}}$ kit, Ipsogen).

Results: The percentage of mutant alleles ranged from 0.0004 to $0.059 \%$, with a median at $0.0045 \%$ (SD 0,0075) and a 95th percentile at $0.019 \%$.

Conclusion: This study contributes to the current efforts aimed at determining JAK2 ${ }^{\mathrm{V} 617 \mathrm{~F}}$ positivity cut-off. The current international consensus considers $1 \%$ of JAK2 $2^{\mathrm{V} 617 \mathrm{~F}}$ allele as a relevant clinical threshold. Using a highly sensitive test, we have shown that this mutation could be detected only at very low levels in healthy individuals. In an ongoing parallel study, we identified a subgroup of patients diagnosed with clinical and biological abnormalities consistent with the diagnosis of MPN, and in which very low allele burden could be detected, overlapping with JAK2 $2^{\mathrm{V} 617 \mathrm{~F}}$ mutation levels detected in $\mathrm{HI}$ These observations need to be confirmed in larger studies, but should provide additional clues into the molecular pathophysiology of these diseases, and help refine current diagnostic criteria.

Disclosure: No conflict of interest disclosed.

\section{V758}

Chronic myelomonocytic leukemia (CMML) is characterized by frequent alterations in TET2, RUNX1, $C B L$, and RAS: A next-generation deep-sequencing study reveals a characteristic pattern of molecular mutations in $72.8 \%$ of patients

Kohlmann, A. ${ }^{1}$; Grossmann, V. ${ }^{1}$; Klein, H.-U. ${ }^{2}$; Haferlach, C. ${ }^{1}$; Schnittger, S. ${ }^{1}$; Kern, W. ${ }^{1}$; Dugas, M. ${ }^{2}$; Haferlach, $T^{1}$

${ }^{1} \mathrm{MLL}$ Münchner Leukämielabor GmbH, München, Germany, ${ }^{2}$ Department of Medical Informatics and Biomathematics, University of Münster,

Münster, Germany

Introduction: Chronic myelomonocytic leukemia (CMML) is a clonal hematopoietic malignancy that is characterized by features of both a myeloproliferative neoplasm and a myelodysplastic syndrome. Clonal cytogenetic abnormalities are found in only 20-30\% of patients with CMML, but none is specific. Further, the molecular background of CMML is poorly understood. Methods: Here, we analyzed 81 thoroughly characterized CMML cases by next-generation sequencing (NGS) (Roche/454 Life Sciences). By designing 43 PCR amplicons targeted re-sequencing allowed us to investigate $C B L$ $J A K 2, M P L, N R A S$, and KRAS at known mutational hotspot regions. In addition, complete coding regions were analyzed for RUNX1 and TET2.

Results: Cytogenetic aberrations were found in $18.2 \%$ (14/77) cases. In contrast, at least one molecular mutation was observed in $72.8 \%(59 / 81)$ of patients using NGS. The median number of generated reads per amplicon was 846 (coverage range 502-fold to 1977-fold). Therefore, this approach allowed a detection of molecular mutations in an unprecedented sensitivity, e.g. detecting the $J A K 2 \mathrm{~V} 617 \mathrm{~F}$ mutation down to $1.16 \%$ of reads. Moreover, ultra-deep amplicon sequencing enabled the discrimination of subclones in a given population as demonstrated for a patient harboring two distinct $K R A S$-mutated clones. In total, 105 variances were detected. After excluding known polymorphisms or silent mutations, 82 distinct mutations remained. TET2 was the most frequently mutated gene: 41 distinct aberrations, i.e. 35 point mutations (19 missense and 17 nonsense) and 6 deletions (2-19 bp), were found in 36/81 $(44.4 \%)$ cases. Of the six deletions, five led to a frameshift. As such, the observed TET2 mutations were found to be heterogeneous and were spread over several exons, predominantly located in the two conserved regions. With respect to clinical data a better outcome was seen for patients that carried TET2 mutations ( $\mathrm{p}=0.013)$. Other mutations observed were as follows: $C B L$ : $\mathrm{n}=15 ; J A K 2$ V617F: $\mathrm{n}=8 ; M P L$ : none; and RUNX1: $\mathrm{n}=7$. Interestingly, we detected $30.8 \%$ mutations in NRAS and KRAS combined (NRAS: $\mathrm{n}=10 ;$ KRAS: $\mathrm{n}=12$ ), with up to four distinct RAS pathway alterations in a single patient. Conclusions: The number of molecular markers used to categorize myeloid neoplasms is constantly increasing. Massively parallel sequencing of single DNA molecules has been demonstrated to support a comprehensive characterization of the molecular background in CMML.

Disclosure: Alexander Kohlmann: Employment or Leadership Position: Wissenschaftlicher Mitarbeiter bei der MLL Münchner Leukämielabor $\mathrm{GmbH}$; Honoraria: Roche Diagnostics GmbH

Torsten Haferlach: Employment or Leadership Position: Geschäftsführer und Teilhaber: MLL Münchner Leukämielabor GmbH; Financing of Scientific Research: Forschungsunterstützung durch Roche Diagnostics GmbH

\section{V759}

Novel PDGFRA point mutations in hypereosinophilic syndrome induce growth factor independence and leukemia in vitro and in vivo

Elling, C. 1; Erben, P.2; Walz, C. ${ }^{2}$; Frickenhaus, M. ${ }^{1}$; Schemionek, M. ${ }^{1}$; Stehling, M. ${ }^{3}$ 'Serve, H. ${ }^{4}$ Cross, N.C.P. ${ }^{5}$. Hochhaus, A. ${ }^{6}$; Hofmann, W.-K. ${ }^{2}$; Berdel, W.E. ${ }^{1}$; Müller-Tidow, C. ${ }^{1}$; Reiter, A. ${ }^{2}$; Koschmieder, S. ${ }^{1}$

${ }^{1}$ Uniklinik Münster, Medizinische Klinik A (Hämatologie/Onkologie), Münster, Germany, ${ }^{2}$ Universitätsmedizin Mannheim, Universität Heidelberg, III. Medizinische Klinik, Mannheim, Germany, ${ }^{3}$ Max-PlanckInstitut für Molekulare Biomedizin, Münster, Germany, ${ }^{4}$ Uniklinik Frankfurt, Medizinische Klinik II, Frankfurt, Germany, ${ }^{5}$ University of Southampton School of Medicine, Human Genetics Division, Southampton, United Kingdom, ${ }^{6}$ Universitätsklinikum Jena, Abt. Hämatologie/Onkologie, Jena, Germany

Introduction: Hypereosinophilic syndrome (HES) is a rare disorder that can be divided into lymphocytic variant $(20 \%)$, myeloproliferative $(20 \%)$, or idiopathic $(60 \%)$ subtypes. The finding of a clonal abnormality such as FIP1L1PDGFRA or other tyrosine kinase fusion genes enables presumptive HES cases to be diagnosed with chronic eosinophilic leukemia. Since most HES patients lack FIP1L1-PDGFRA, we studied whether activating mutations of PDGFRA can be detected in this group of patients.

Methods: The PDGFRA coding region (exons 9-19) was sequenced in 54 idiopathic HES patients without known fusion genes. PDGFRA point mutants were cloned and retrovirally transduced into murine myeloid 32D cells. These cells were analyzed for IL-3 independent growth in liquid culture and CFU assays, activation of downstream signaling pathways using Western blotting, and leukemia development in vivo after injection into congenic $\mathrm{C} 3 \mathrm{H} / \mathrm{HeJ}$ mice. Imatinib sensitivity was analyzed in vitro and in vivo.

Results: Sequencing of the PDGFRA gene revealed point mutations in 9 of the 54 patients. Four patients harbored mutations at position $849(n=3)$ or 659 $(\mathrm{n}=1)$, and activating point mutants involving these amino acids have been previously described in gastrointestinal stromal tumors. In addition, we detected several novel single (R481G, L705P, G729D) or double mutations (H650Q + R748G, I562M + H570R) in one patient each. When cloned into 32D cells, H650Q and R748G mutants separately or together but not the other mutants induced growth factor-independent proliferation and clonogenic growth, and constitutive phosphorylation of the downstream targets STAT5 
and AKT. Low doses of imatinib $(0.5 \mu \mathrm{M})$ antagonized all of these effects in vitro. H650Q and R748G 32D cell mutants induced leukemia after injection into syngenic $\mathrm{C} 3 \mathrm{H} / \mathrm{HeJ}$ mice, similar to FIP1L1-PDGFRA. Interestingly, these two mutants showed a significantly higher propensity to invade the lymph nodes compared to the FIP1L1-PDGFRA fusion. Oral imatinib treatment of injected mice significantly decreased leukemic growth in vivo and significantly prolonged survival of the recipients

Conclusion: In conclusion, our data about novel activating point mutations of the PDGFRA gene suggest that this gene should be sequenced in all patients with HES and that these patients may benefit from imatinib treatment.

[CE, PE, AR, and SK contributed equally]

Disclosure: Christian Elling: No conflict of interest disclosed.

Steffen Koschmieder: Advisory Role: BMS, Novartis; Honoraria: Novartis; Financing of Scientific Research: Novartis; Other Financial Relationships: Novartis, BMS

\section{V760}

\section{Analysis of 418 Patients with different myeloproliferative neoplasms revealed a strong association of CBL mutations with the CMML}

Schnittger, S. ${ }^{1}$; Reiter, A. ${ }^{2}$; Dicker, F.1. Ulke, M. ${ }^{1}$; Spiel, A. ${ }^{1}$; Kern, W. ${ }^{1}$; Haferlach, C. ${ }^{1}$; Haferlach, T.

${ }^{1}$ MLL Münchner Leukämie Labor, München, Germany, ${ }^{2}$ Universitätsmedizin Mannheim, III. Medizinische Klinik, Mannheim, Germany

Mutations in the CBL gene targeting the RING and linker domains have recently been described in CMML, atypical CML and single cases of PMF or HES. To further analyse the incidence of CBL mutations in myeloproliferative neoplasms (MPN) we analyzed 418 patients with different MPN. This cohort was selected according to the availability of cytomorphology, cytogenetics and further molecular genetic characterization. A BCR-ABL1 rearrangement was excluded in all cases. Analysis of the relevant region of CBL (exons 8 and 9 ) revealed mutations in $26 / 418$ cases $(6.2 \%)$. All mutations were missense mutations and were clustered in a region spanning 50 amino acids around the RING and linker domains. Two of 28 mutations were detected in two cases each (C419Y and A420G), all others were single and as follows (L380P, L381G, L381P, I383M, C384T, I384T, D388G, D390V, C396Y, G397V, H398A, C404Y, W408C, C416R, G415S, C416S, C416Y, P417H, R420L, R421L, I423N, I429F, I429N, V430M). In 12 /26 cases the absence of a CBL wildtype (wt) indicated allelic loss. Two patients had two different mutations. The mutations were most frequently found in CMML-1 (14/73; 19.2\%), CMML-2 $(2 / 26 ; 7.9 \%)$, PMF $(4 / 32 ; 12.5 \%)$ and MPN unclassifiable $(7 / 99 ; 7.1 \%)$. In contrast, CBL mutations were not detected in PV, ET, HES, RARS-T and MDS/MPN overlap. The presence of CBL mutations was not correlated to age, gender, chromosomal aberrations or WBC. All $26 \mathrm{CBL}$ mutated cases were analysed for JAK2V617F, JAK2exon12, MPLW515, FLT3-ITD, MLL-PTD, NPM1, NRAS and RUNX1 mutations. The CBL mutations were exclusive of all these mutations with the exception of RUNX1 and TET2. Four of 16 CMML cases $(25 \%)$ with CBL mutations had a RUNX1 mutation and one MPNu case revealed a TET2 mutation. In CMML-1 11/14 (78.6\%) cases showed loss of the wt allele or two different mutations and thus lack a functionally intact CBL allele. In contrast, nearly all CBL mutations in MPNu and all PMF had a low allelic burden. In conclusion, these data show that CBL mutations i) are highly correlated with CMML, ii) in CMML are associated with high allelic burden or two CBL mutations, iii) in CMML are frequently associated with RUNX1 mutations, iv) are rarely found in PMF and MPNu and are rare or absent in all other MPN, v) seem to be mutually exclusive of other mutations typical for MPN such as JAK2 and MPLW515 mutations. Thus patients with suspected MPN who lack other MPN typical mutations should be routinely analyzed for CBL mutations

Disclosure: Susanne Schnittger: Employment or Leadership Position: MLL Münchner Leukämielabor

Torsten Haferlach: Employment or Leadership Position: MLL Münchner Leukämielabor
V761

\section{Relapse-therapy for myelofibrosis patients after allogeneic stem cell transplantation}

\section{Klyuchnikov, E. ${ }^{1}$; Alchalby, H. ${ }^{1}$; Badbaran, A. ${ }^{1}$; Wolschke, C. ${ }^{1}$;} Ayuk, F. ${ }^{1}$; Bacher, U. ${ }^{1}$; Zander, A. ${ }^{1}$; Kröger, N. ${ }^{1}$

${ }^{1}$ Universitätsklinikum Hamburg-Eppendorf, Interdisziplinäre Klinik und Poliklinik für Stammzelltransplantation, Hamburg, Germany

Introduction: Up to $30 \%$ of patients with myelofibrosis (MF) will relapse within the first three years after allo-SCT. Therapeutic options for those patients include donor lymphocyte infusions (DLIs) and/or a $2^{\text {nd }}$ allo-SCT, but studies on this issue are rare.

Patients and methods: We report our experience with DLIs and $2^{\text {nd }}$ allo-SCT being performed as salvage approach in $10 \mathrm{pts}(5 / \mathrm{m}, 5 / \mathrm{f})$ with relapsed MF (32-63 yrs). Initial transplantations were performed in all pts with reduced conditioning (RIC) (busulfan, cum. $10 \mathrm{mg} / \mathrm{kg}$ p.o. or equivalent i.v.; + fludarabine, cum. $180 \mathrm{mg} / \mathrm{m}^{2}$ ). Maximal response after initial allo-SCT was complete $(\mathrm{CR}, \mathrm{n}=3)$ or partial remission $(\mathrm{PR}, \mathrm{n}=6)$, and stable disease $(\mathrm{SD}, \mathrm{n}=1)$.

Results: Clinical relapse occurred at a median of 5 months (2-50) following initial allo-SCT. Subsequently, all pts received DLIs at 1-4 time points (median, $2.5 \times 10^{7} / \mathrm{kg}$ bw; range, $0.5 \times 10^{5}-1.3 \times 10^{8} / \mathrm{kg}$ ). Thereafter, 2 pts $(20 \%)$ developed grade III acute graft versus host disease (aGvHD) and required adequate immunosuppression. The best responses after DLIs according to IWG-MRT criteria included: CR $(n=3), S D(n=5)$, and clinical improvement $(\mathrm{CI}, \mathrm{n}=1)$. One pt showed progressive disease $(\mathrm{PD})$. All pts with $\mathrm{CR}$ maintain CR 7+, 18+ and 19+ months. $2^{\text {nd }}$ allo-SCT was performed in 7 pts without CR following DLIs. The median interval between both SCTs was 17 months (13 - 77). RIC regimen being used in this setting consisted of fludarabine (cum. $150 \mathrm{mg} / \mathrm{m}^{2}$ ) with treosulfan (cum. $36 \mathrm{mg} / \mathrm{m}^{2}$ ). All pts received PBSC (median, $7 \times 10^{6} \mathrm{CD}^{2} 4^{+}$cells/kg bw; 4 - 11) from alternative HLA-matched $(\mathrm{n}=3)$ or -mismatched $(n=4)$ unrelated donors. All pts successfully achieved leukocyte engraftment (median, day $+18 ; 10-20$ ). Severe aGvHD (II-III) was observed in 2 of $7(29 \%)$ pts. There were no TRM cases. Of all 10 pts, 8 were alive at the median follow up of 34 months (range, 16-78) from initial SCT. Of those, according to IWG-MRT criteria, 6 achieved CR, together with JAK2V617Fnegativity ( 3 pts after DLIs and 3 pts after $2^{\text {nd }}$ allo-SCT, including the pt with PD after the $1^{\text {st }}$ allo-SCT). 2 patients attained PR following the $2^{\text {nd }}$ SCT. Two of 10 pts who were in CR 15 and 11 months after the $2^{\text {st }}$ allo-SCT, respectively, died due to PD 18 and 21 months after the $2^{\text {nd }}$ SCT.

Conclusions: DLIs and $2^{\text {nd }}$ allo-SCT, based on a combination of fludarabine and treosulfan, seem to represent effective and well tolerated salvage approaches for patients with myelofibrosis, who relapsed after initial allo-SCT.

Disclosure: No conflict of interest disclosed

\section{V762}

\section{Critical evaluation of current WHO diagnostic criteria for aggressive systemic mastocytosis}

Metzgeroth, G. ${ }^{1}$; Erben, P. ${ }^{1}$; Bolz, G. ${ }^{1}$; Teichmann, M. ${ }^{1}$; Popa, J.'; Michaely, H. ${ }^{2}$; Ströbel, P. $^{3}$; Marx, A. ${ }^{3}$; Horny, H.-P.4; Hochhaus, A. ${ }^{5}$; Hofmann, W.-K. ${ }^{1}$; Reiter, A.

${ }^{1}$ III. Medizinische Klinik, Universitätsmedizin Mannheim, Mannheim, Germany, ${ }^{2}$ Institut für Klinische Radiologie und Nuklearmedizin, Universitätsmedizin Mannheim, Mannheim, Germany, ${ }^{3}$ Pathologisches Institut, Universitätsmedizin Mannheim, Mannheim, Germany, ${ }^{4}$ Institut für Pathologie, Ansbach, Germany, ${ }^{5}$ Klinik für Innere Medizin II,

Universitätsklinikum Jena, Jena, Germany

According to the current WHO classification, diagnosis of aggressive systemic mastocytosis (ASM) is met when mast cell infiltrates in at least one extracutaneous tissue are found and at least one of the $\mathrm{C}$-findings is present: neutrophils $<1000 / \mu \mathrm{l}, \mathrm{Hb}<10 \mathrm{~g} / \mathrm{dL}$, platelets $<100 / \mathrm{nl}$, impaired liver function with ascites, splenomegaly with hypersplenism, large osteolytic lesions or malabsorption with significant weight loss of $>10 \%$ in 6 months. In the present study, we evaluated the diagnostic value of these parameters in 26 ASM patients (pts). Median age was 67.5 years (range, 30-82) with a male:female ratio of 1.4:1. All pts had a KITD816V mutation and an elevated serum tryptase. Anemia and thrombocytopenia were present in 10 of $25(40 \%)$ and 15 of $25(60 \%)$ pts, respectively, while neutropenia was not observed. 
Monocytosis $(>1000 / \mu \mathrm{l})$ and eosinophilia $(>500 / \mu \mathrm{l})$ were seen in 10 of 22 (45\%) and 14 of $21(65 \%)$ pts. Median BM mast cell infiltration was $40 \%$ (range, 15-85). 16 of 25 (64\%) pts were diagnosed as ASM+AHNMD CMML-1, n=8; HES/CEL, n=3; MDS/MPN, n=2; MDS-RCMD, n=1; MDSRAEB-1, n=1; PMF, $n=1$. Splenomegaly was seen in 22 of $26(85 \%)$, lymphadenopathy in 17 of $24(71 \%)$ and ascites in 12 of $25(48 \%)$ pts, respectively. We observed elevation of ASAT in 3 of 24 (13\%), ALAT in 3 of $25(12 \%)$ or bilirubin in 2 of $25(8 \%)$ pts only. In contrast, increased AP and gGT was seen in 20 of $25(80 \%)$ and 20 of $26(77 \%)$ pts, respectively. Additional relevan laboratory markers were low serum albumine $(<35 \mathrm{~g} / 1)$ in 9 of $23(39 \%)$, decreased prothrombin time (PT, $<60 \%)$ in 12 of $23(52 \%)$ and low cholesterol $(<130 \mathrm{mg} / \mathrm{dL})$ in 16 of $22(73 \%)$ pts. Only a minority of pts revealed increase of LDH $(4 / 25,16 \%)$ or uric acid $(3 / 24,13 \%)$. Frequently observed clinicial symptoms included significant weight loss $(13 / 20,65 \%)$, diarrhea $(12 / 22,55 \%)$ and urticaria pigmentosa $(8 / 23,35 \%)$. Osteolytic bone lesions were detected by MRI and/or X-ray only in 4 of $23(17 \%)$ pts. All osteolyses were small and never exceeded $15 \mathrm{~mm}$. In summary, the diagnostic value of the clinical and laboratory parameters of the current WHO diagnostic criteria for ASM is highly variable. Neutropenia and large osteolytic lesions are virtually absent while the frequency of lymphadenopathy and urticaria pigmentosa is underestimated. Elevated AP and gGT, decreased PT and low cholesterol are clearly superior markers as compared to ASAT, ALAT and bilirubin. We conclude that an update of diagnostic criteria for ASM is warranted.

Disclosure: No conflict of interest disclosed.

\section{Expertenseminar}

Therapieoptionen in der Behandlung refraktärer maligner Lymphome - ein fallorientiertes Expertenseminar

\section{V770}

\section{Therapeutic options in relapsed peripheral T-cell lymphoma}

\section{Wulf, G. G. ${ }^{1}$}

${ }^{1}$ University of Göttingen, Hematology / Oncology, Göttingen, Germany

The prognosis of patients with PTCL is still dismal, particularly in the situation of progressive or relapsed disease. Current evidence is gained from case series treated within or in parallel to clinical trials for patients with aggressive B-cell lymphoma, and is emerging from recent phase I and II trials. For eligible patients, intensive salvage therapy integrating high-dose consolidation with autologous or allogeneic stem cell transplantation (SCT) should be evaluated. Widely used salvage regimens are platinum-based combinations such as ICE or DHAP. Allogeneic SCT can induce graft-versus-lymphoma activity in PTCL, leading to PFS rates at 3 years between $30 \%$ to $60 \%$, both in phase II trials and larger registry analyses. Reduced-intensity conditioning (RIC) has provided encouraging low rates of treatment related morbidity and mortality. However, lacking comparative studies between myeloablative versus RI conditioning, the choice of intensity currently has to weigh disease control versus comorbidities for the individual patient. High-dose therapy with BEAM followed by autologous SCT is an option for patients, who had not received autologous SCT as first line therapy, those ineligible for allogeneic transplantation, and/or those with longer disease free interval prior to recurrence. Among novel therapeutic principles, histone deacetylase inhibitors (HDAC-I) represent a consistently active class of small molecules with overall response rates (ORR) of 30\% in T-cell lymphoma, leading to FDA approval for refractory or relapsed CTCL for both romidepsin and vorinostat. For PTCL, the HDAC-I belinostat is currently available within a phase II trial at several clinical trial centers. The humanized monoclonal antibodies alemtuzumab against CD52, and zanolimumab against CD4 have activity against relapsed PTCL as single agents; however, the use of both agents is not covered by EMEA approval in PTCL. For patients with CD30 positive anaplastic large cell lymphoma (ALCL), the immunotoxin SGN35 has shown promising results similar to the situation in relapsed Hodgkin's disease. The folate analog pralatrexate, targeting the reduced folate carrier particularly in PTCL lymphoma cells, has shown single agent activity with $30 \%$ ORR, and has been granted FDA approval in relapsed or refractory PTCL. Integrating advances in SCT as well as targeted therapy in novel combination regimens can reasonably be expected to improve the prospects of patients with PTCL.

Disclosure: No conflict of interest disclosed.

\section{Posterdiskussion AML experimentell \\ P774 \\ Carboxy-terminal phosphorylation of SIRT1 by protein kinase CK2}

\section{Mahlknecht, U. ${ }^{1} ;$ Zschörnig, B. ${ }^{1}$}

${ }^{1}$ Universität des Saarlandes, Innere Medizin, Jose Carreras Zentrum für Immuntherapie und Gentherapie, Homburg/Saar, Germany

Introduction: Gene expression and deacetylase activity of the class III histone deacetylase SIRT1 are up-regulated in malignant cells. Previous analyses of the sirtuin family of histone deacetylases and its most prominent member SIRT1have focused primarily on the identification of cellular targets exploring the underlying molecular mechanisms of its implicated function in the control of metabolic homeostasis, differentiation, apoptosis and cell survival. SIRT1 may induce both, histone deacetylation and methylation of $\mathrm{CpG}$ islands within the promoter regions of specific genes, thus mediating transcriptional repression, and deacetylation of tumor suppressor proteins. SIRT1 plays a critical role in tumor initiation and progression and promotes cell growth and angiogenesis. SIRT1 inhibitors have shown promising anticancer effects in animal tumor models.

Methods: Sirt1 amino acid sequence analyses revealed several putative CK2 phosphorylation sites predominantly located within the C-terminal domain. We generated various deletion mutants expressing sequential C-terminal truncations of FLAG-tagged SIRT1 at amino acid positions 643, 595 and 527 followed by ${ }^{32} \mathrm{P}$-labeling. Immunoprecipitated FLAG-tagged SIRT1 from transfected HeLa cells was incubated with recombinant CK2 holoenzyme in the presence of [y-32]ATP and CK2 efficiently phosphorylated SIRT1 in vitro.

Results: In the study presented herein, we assigned the main region of SIRT1 in vivo phosphorylation to amino acids 643-691 of the unique carboxy-terminal domain. Furthermore, we demonstrate that SIRT1 is a substrate for protein kinase CK2 both in vitro and in vivo. Both, deletion construct analyses and serine-to-alanine mutations identified SIRT1 Ser-659 and Ser-661 as major CK2 phosphorylation sites that are phosphorylated in vivo as well.

Conclusions: So far, little is known about the regulation of SIRT1 itself. CK2 takes part in the same cellular processes as SIRT1. In addition, C-terminal phosphorylation of SIRT2 and most recently of SIRT1 by cyclin-dependent kinases has been demonstrated to be key elements during cell cycle progression. Many of the regulatory mechanisms described so far, depend on the unique amino and carboxy-terminal extensions of SIRT1.

Disclosure: No conflict of interest disclosed.

\section{P775}

\section{RASSF1A as a novel target of microRNA $181 \mathrm{~b}$ in acute} promyelocytic leukemia

\section{Bräuer, D. ${ }^{1}$; Hartmann, J.-U.1; Gerloff, D. ${ }^{1}$; Christopeit, M. ${ }^{1}$; Behre, G.}

${ }^{1}$ Martin-Luther-Universität Halle-Wittenberg, Universitätsklinikum, Klinik für Innere Medizin IV, Landeszentrum für Zell- und Gentherapie, Halle, Germany

Introduction: In the acute promyelocytic leukemia (APL) bearing the $\mathrm{t}(15 ; 17)$, all-trans-retinoic acid (ATRA) treatment induces granulocytic maturation and complete remission of leukemia. Several factors are involved in the formation of the leukemic phenotype. Latest studies identified microRNAs as critical players in this network.

Results: In a micro array based microRNA screen we could identify miR $181 \mathrm{~b}$ as downregulated in the APL cell line NB4 by treatment with pharmacological doses of ATRA. Additionally, we showed the transcriptional induction of miR-181b by the APL-associated PML-RAR $\alpha$ oncogene which is released after treatment with ATRA. The overexpression of miR-181b by microRNA mimics leads to an inhibition of ATRA induced granulocytic differentiation. Furthermore, the downregulation of miR-181b by locked nucleic acids (LNAs) causes the induction of granulocytic transcription factors $\mathrm{C} / \mathrm{EBP} \alpha$ and $\mathrm{C} /$ EBP $\beta$. In a microRNA target search we identified the novel ATRA regulated 
tumorsupressor RASSF1A as a putative target of miR-181b. In functional studies we showed that enforced expression of miR-181b reduces the protein level of RASSF1A by binding to the 3'UTR of RASSF1A mRNA. Accordingly, RASSF1A protein was enriched by the knock down of miR-181b. The role of RASSF1A in ATRA induced differentiation was verified by knock down of RASSF1A protein by specific siRNA. Here we could show the reduction of ATRA induced CD $11 \mathrm{~b}$ expression.

Conclusion: We identified miR-181b as a new player in the PML/RARo associated APL. Moreover, we firstly described the miR-181b target RASSF1A as a crucial factor in the ATRA activated granulocytic differentiation program in APL. These data reveal the importance of deregulated miRNA biogenesis in cancer and may provide novel biomarkers and therapeutic targets in myeloid leukaemia.

Disclosure: No conflict of interest disclosed.

\section{P776}

Role of the PDK1-AKT axis in acute leukemia - A potential resistance mechanism towards receptor tyrosine kinase (RTK) targeted therapies

Kampa-Schittenhelm, K.M. ${ }^{1}$; Mueller, S. ${ }^{1}$; Kanz, L. ${ }^{1}$; Heinrich, M. ${ }^{2}$; Schittenhelm, M. ${ }^{1}$

${ }^{1}$ Universitätsklinikum Tübingen, Med. II Hämatologie, Onkologie, Rheumatologie, Immunologie, Pulmologie, Tübingen, Germany,

${ }^{2}$ Departments of Cell and Developmental Biology, Division of Hematology/ Oncology, Oregon Health and Science University Cancer Institute, and the Portland Veterans Affairs Medical Center, Portland, United States

Introduction: A significant subgroup of acute leukemias harbors activating mutations in class III RTKs which fuel leukemogenesis. The most prevalent gain-of-function mutations in acute leukemias (i.e. FLT3 ITD, KIT D816 and $\mathrm{BCR} / \mathrm{ABL}$ ) are associated with poor outcome. Clinical studies demonstrated the significant tumor reductive potential of small molecule tyrosine kinase inhibitors (TKI), but responses are rather short or moderate. We here demonstrate that signaling via the PDK1-AKT axis contributes to leukemogenesis and enables evasion of acute leukemia from TKI monotherapy.

Methods: Signal transduction in the presence or absence of different TKI (dasatinib, imatinib, sunitinib, sorafenib) was analyzed in AML cell lines harboring common gain-of-function mutations and blasts of leukemia patients using FACS and immunoblotting. Involvement of PDK1 signaling via pAKT(Thr308) in leukemogenesis was evaluated in vivo by analysis of tumor formation of stably PDK1-silenced vs. mock transfected leukemia cells in NOD/SCID mice. The efficacy of TKI in combination with AKT-pathway inhibitors like the PI3K/mTOR inhibitor NVP-BEZ235 was assessed by apoptosis and proliferation assays.

Results: FLT3, KIT or BCR/ABL-inhibition does not sufficiently silence AKT signaling in acute leukemias as (i) threonine phosphorylation of AKT a codon 308 by PDK1 remained unaffected and (ii) downstream AKT signaling remained active as revealed by FACS, Western Blot and phosphoproteome profiling. In line, following i.v. injection of $5 \times 10^{6}$ leukemic cells, tremendously higher peripheral blast counts after 8 days were observed in control mice $\left(\sim 4 \times 10^{3} / \mu \mathrm{L}\right)$ compared to the PDK1-silenced group $\left(\sim 0.5 \times 10^{3} / \mu \mathrm{L}\right)$. Of note, targeting the mTOR/PDK1 pathway using NVP-BEZ235 potently induced apoptosis and inhibited proliferation of leukemia cells harboring various FLT3 and KIT isoforms with an IC50 of 500-1000 nM. Combination with TKI potentiated the antiproliferative and proapoptotic activity of either monosubstance.

Conclusion: Our data identify the PDK1-AKT axis as a crucial contributor to leukemogenesis, potentially contributing to resistance towards TKI therapy in acute leukemia. Clinically, combination of TKI with NVP-BEZ235 may serve to overcome resistance to RTK-targeted therapy in acute leukemia.

Disclosure: No conflict of interest disclosed.

\section{P777}

\section{Osteopontin as a prognostic factor in acute myeloid leukemia}

Liersch, R. ${ }^{1}$; Biermann, C. ${ }^{1}$; Appelmann, I. ${ }^{1}$; Schliemann, C. ${ }^{1}$; Kessler, T. ${ }^{1}$; Bayer, M. ${ }^{1}$; Schwöppe, C. ${ }^{1}$; Müller-Tidow, C. ${ }^{1}$; Berdel, W.E. ${ }^{1}$; Mesters, R.M. ${ }^{1}$

${ }^{1}$ Universitätsklinikum Münster, Medizinische Klinik und Poliklinik A, Münster, Germany

Introduction: Osteopontin (OPN) is a secreted glycoprotein that is widely expressed in various kinds of cells and is involved in normal tissue remodelling processes as well as in certain diseases such as tumorigenesis and tumor metastasis. In the bone marrow (BM) OPN is predominantly secreted by osteoblasts and hematopoietic cells, which have been shown recently to express the OPN-binding integrins alpha4beta1 and alpha9beta1. In addition, OPN has been defined as an important factor for hematopoietic stem cells (HSCs). OPN suppressed the proliferation of HSCs in vitro and may regulate the hematopoietic stem cell pool. Increased serum OPN concentrations have been reported in chronic myeloid leukemia (CML), multiple myeloma (MM) and acute myeloid leukemia (AML).

Methods: We investigated the expression of OPN in newly diagnosed AML patients by immunohistochemistry $(n=84)$, enzyme-linked immunoassays (ELISA) of blood /bone marrow sera $(n=40)$ and on the RNA level by analyzing microarray data $(n=261)$.

Results: Expression of OPN was increased in AML patients bone marrow sera (ELISA) as well as in bone marrow blasts (IHC) Patients expressing high levels of OPN within the bone marrow (IHC: $>10$ arbitrary units [AU]; ELISA: $>10 \mathrm{ng} / \mathrm{ml}$ ) had significantly shorter overall survival (OS) than those with lower OPN levels. In contrast, blood OPN levels showed no predictive value. There was no correlation found between OPN expression and FABsubtypes M0 to M7 or different karyotypes. Multivariate analysis identified the already known risk factors karyotype, blast clearance (day 16) and the level of OPN expression as independent prognostic factors for OS. Furthermore, analyses of microarray data from 261 patients of a different cohort confirmed OPN as a prognostic marker. In detail, high OPN expression demonstrated a negative predictive value for EFS and OS. Subgroup analysis revealed a significant difference in EFS and OS for OPN levels above the median in FLT3ITD/TKD mutation negative leukemias, only. No difference was found in FLT3-mutated leukemias or in patients with favorable cytogenetics such as $\mathrm{t}(8 / 21)$ or inv (16)

Conclusions: These data provide evidence for OPN as prognostic marker in AML. OPN might be of pathogenetic relevance in AML. Although the mechanism is not yet understood modulation of the OPN axis might be a promising approach to improve the outcome of AML patients in the future.

Disclosure: No conflict of interest disclosed.

\section{P778}

Induction of immunogenic cell death through cellular delocalization of calreticulin in acute myeloid leukemia (AML)

\section{Mans, S. ${ }^{1}$; Klebig, C. ${ }^{1}$; Shan, D. ${ }^{1}$; Mueller, B.U. ${ }^{2}$; Pabst, T. ${ }^{3}$}

${ }^{1}$ Departement für klinische Forschung, Universität Bern, Bern, Switzerland, ${ }^{2}$ Klinik für Allgemeine Innere Medizin, Universitätsspital, Bern, Switzerland, ${ }^{3}$ Departement für Medizinische Onkologie, Universitätsspital, Bern, Switzerland

The RNA-binding protein calreticulin (CRT) inhibits translation of the CCAAT/enhancer binding protein-alpha (CEBPA) leading to a differentiation block of myeloid progenitors. CRT is a resident protein of the endoplasmatic reticulum (ER) and involved in the unfolded protein response (UPR) which is triggered by accumulation of misfolded proteins in the ER. Previous reports in solid tumors indicated that ER stress can be induced by anthracycline treatment activating the UPR, ultimately leading to CRT exposure at the outer cell surface. This ecto-CRT is targeting dendritic cells and can initiate immunogenic cell death of cancer cells by acting as an "eat-me" signal. Although anthracyclines, in combination with cytarabine, represent the standard induction therapy for AML patients, ER stress induced CRT exposure has not been investigated so far in leukemogenesis. This study aimed to analyze anthracy- 
cline induced exposure of CRT in myeloid leukemic cells, thereby possibly contributing to the eradication of chemotherapy-resistant cancer cells. We found that anthracycline treatment in vitro increased mRNA expression of initiating effectors of ER stress including CRT. FACS analyses indicated exposure of CRT at the surface of leukemic HL60 cells already after three hours of incubation with doxorubicin. In AML patients, we observed a significant increase of CRT exposure at the cell surface of myeloid blasts compared to granulocytes of healthy controls. Furthermore, we established an $\mathrm{N}$ - and a C-terminal specific CRT ELISA to determine secreted CRT levels in the serum. The measurement of serum samples from 70 AML patients and 27 healthy controls showed a significant increase of the N-terminal CRT in AML patients at diagnosis vs. healthy controls. Measurement of CRT levels in patients achieving complete remission vs. refractory patients indicated a trend of increased CRT concentration (n.s.) to be associated with a more favorable clinical outcome. Measurement of serum CRT concentration during treatment of patients suggests that chemotherapy selectively induced N-terminal CRT peptide levels. In summary, our observations indicate that CRT can be translocated to the cell surface and ultimately be secreted to the serum in response to induction of ER stress following anthracycline treatment in AML. Ongoing studies intend to ultimately identify CRT exposure as a predictive marker for response and outcome to anthracycline treatment in AML patients.

Disclosure: No conflict of interest disclosed.

\section{P779}

\section{Identification of AML-subtype specific HDAC gene expression patterns and its clinical impact}

\section{Dransfeld, C.-L. ${ }^{1}$; Schaich, M. ${ }^{2}$; Ehninger, G. ${ }^{2}$; Ong, M.F.3; Mahlknecht, $U .^{1}$ \\ ${ }^{1}$ Universität des Saarlandes, José Carreras Zentrum für Immuntherapie und Gentherapie, Homburg, Germany, ${ }^{2}$ Universität Dresden, Innere Medizin I, Dresden, Germany, ${ }^{3}$ Universität des Saarlandes, Institut für medizinische Biometrie, Homburg, Germany}

Introduction: Current clinical research in acute myeloid leukemia (AML) focuses on drugs targeting unique genomic aberrations, fusion proteins, or misregulated cellular pathways that are characteristic for molecular subgroups of patients, in order to achieve a more and more individualized, subtype specific therapy.

In a number of ongoing pathogenesis models for AML and myelodysplastic syndromes (MDS) the recruitment of Histone deacetylases (HDACs) is considered as a crucial event and therefore the basis for innovative epigenetic treatment strategies. The use of HDAC-inhibitors actually takes part of these new therapeutic strategies and is analyzed in many recent studies. Consequently it is important to take into account differences in the expression levels of HDACs in AML subtypes, which could hardly affect the impact of therapy. Furthermore HDAC expression levels could serve as additional diagnostic markers of AML subtypes.

Methods: Having analyzed the putative correlation between SNPs in HDACs and the susceptibility for AML (Dransfeld et al. 2007), we now performed PCR based subtype specific gene expression analysis of HDACs in 54 AML patients including probes of every frequent AML subtypes (except M3) and different, most characteristic karyotypes cytogenetics, compared to the mean values healthy donors. In addition we compared the gene expression levels with the therapy response, to reveal putative relations between HDAC expression levels and therapeutic outcome.

Results: Our results revealed characteristic general and subtype specific expression patterns, like AML M1 as a subtype with maximal over expression of all analyzed HDACs, which could help to further specify AML treatment In addition to that we could reveal significant specific HDAC expression patterns in correlation with distinct cytogenetics.

Interestingly specially class II and III HDACs, which were almost disregarded in previous studies, had been shown to be altered in AML subtypes.

The clinical impact of the distinct HDAC expression pattern of AML subgroups could be emphasized by our findings that they were correlated with complete remission and relapse.

Conclusions: The knowledge of the specific expression levels of HDACs in the diverse subgroups of AML assessed by our studies is an indispensable basis for the upgrading of AML therapy via optimization of HDAC inhibitor doses.

Disclosure: No conflict of interest disclosed.
P780

Polo-like kinase 1 (PIk1) inhibition results in mitotic arrest of leukemic blasts in bone marrow biopsies of patients with acute myeloid leukemia

May, A.M. ${ }^{1}$; Münch, C. ${ }^{1}$; Schöpflin, A. ${ }^{1}$; Lübbert, M. ${ }^{2}$; Wäsch, R. $^{2}$; Taube, T. $^{3}$; Lassmann, S. ${ }^{1}$; Werner, M. ${ }^{1}$

${ }^{1}$ Institut für Pathologie, Universitätsklinikum Freiburg, Freiburg, Germany, ${ }^{2}$ Abteilung für Hämatologie und Onkologie, Universitätsklinikum Freiburg, Freiburg, Germany, ${ }^{3}$ Boehringer Ingelheim Pharma GmbH \& Co. KG, Abteilung Klinische Forschung, Biberach, Germany

Introduction: The mitotic serine/threonine kinase Polo-like kinase 1 (Plk1) is an important regulator of the cell cycle, especially at the G2/M transition and during mitosis. Plk1 is overexpressed in several solid tumors as well as in acute myeloid (AML) and lymphoblastic leukemias, where its suitability as a therapeutic target is currently being evaluated in several clinical trials. In this study we assessed the effects of Plk1 inhibition on the histomorphology of bone marrow biopsies (BMBs) of AML patients treated with BI2536.

Methods: We analyzed pre- and post-treatment BMBs of six AML patients who were treated with the selective, small molecule Plk1 inhibitor BI2536, before and one day after application of variable doses of the compound. We performed routine diagnostic stains (Gomori, iron stain, CAE, Giemsa) and immunohistochemistry for Plk1. Morphological assessment specifically addressed AML blasts.

Results: Compared to pre-treatment BMBs, all post-treatment BMBs displayed an arrest of leukemic blasts in mitosis (increase of mitotic figures: 3 - to 189-fold), with marked atypical mitotic figures. In addition, a striking increase of apoptotic cells was observed in post treatment BMBs (up to 15-fold). However, these effects were dependent on BI2536 dosage: 50-60 mg triggered an up to 10-fold increase of mitoses and up to 5-fold increase of apoptoses, whereas the high dose of $400 \mathrm{mg}$ resulted in an 189-fold increase of mitoses and a 15-fold increase of apoptoses. Moreover, immunohistochemistry demonstrated that the centrosomal and/or spindle pole associated localisation of Plk1 in mitotic figures before therapy, was lost in the atypical mitoses observed in the post-treatment BMBs (weak diffuse cytoplasmic staining).

Conclusions: Our data confirm the sensitivity of AML blasts to Plk1 inhibition with small molecule inhibitors in a dose-depended fashion. We were able to demonstrate that induction of atypical mitoses is due to a failure of accurate intracellular localization of Plk1 protein. Together, the morphological findings of the present study suggest that BI2536 treated AML blasts undergo mitotic catastrophe, further supporting data from cell culture experiments.

Disclosure: No conflict of interest disclosed.

\section{P781}

\section{Specific immune responses against epitopes derived from Aurorakinase A and B}

\author{
Hofmann, S. ${ }^{1}$; Eggenrieder, S. ${ }^{1}$; Götz, M. ${ }^{1}$; Herbst, C. ${ }^{1}$; \\ Doehner, H. ${ }^{1}$; Greiner, J. ${ }^{1}$ \\ ${ }^{1}$ Universität UIm, Klinik für Innere Medizin III, UIm, Germany
}

Targeted immunotherapies require the identification and characterization of appropriate immunogenic antigen structures. To date, several tumor-associated antigens (TAAs) but also leukemia-associated antigens (LAAs) have been identified and attempts have been made to target these antigens as e.g. in peptide vaccination trials against proteinase 3, RHAMM and WT-1. However, antigens like WT-1 and proteinase 3 are expressed in normal tissues including normal CD34-positive cells and are only expressed in a minority of tumor entities.

Aurorakinase A and B are strongly expressed in all proliferating cells and are overexpressed in most acute leukemias but also solid tumors. Aurorakinase A and $\mathrm{B}$ are appropriate target structures in malignant hematological diseases as these antigens are involved in different critical mechanisms for cell differentiation and proliferation and therefore they represent appropriate target structures.

To define T cell epitopes, peptide sequences derived from Aurorakinase A and B with HLA-A 0201-binding motifs were predicted using computer analysis based on known binding affinities. Ten peptides with most favourable characteristics were subjected to ELISPOT assays for interferon gamma and granzyme B in healthy volunteers and AML patients. Twelve healthy volunteers 
and ten AML patients were tested. Four nonamers and two decamers derived from Aurorakinase B and two nonamers derived from Aurorakinase A were tested.

Seven epitopes induced specific $\mathrm{T}$ cell responses in ELISPOT analysis of tested samples. 11/12 healthy volunteers were tested positive. Frequen responses in more than $60 \%$ of healthy volunteers were detected against epitopes derived from Aurorakinase A (epitope A1 and A2) but also agains epitopes derived from Aurorakinase B in 50\% (epitope B1) and 30\% (epitopes B3 and B4). In AML samples, we detected the most frequent $\mathrm{T}$ cell responses against the epitope $\mathrm{A} 2$ in $40 \%$ of patients but also against B4 and B5 in $33 \%$ of AML patients and most patients showed $\mathrm{T}$ cell responses against one of these epitopes.

Taken together, Aurorakinase A and B induce frequent $\mathrm{T}$ cell responses in healthy volunteers and AML patients. Due to the expression in most acute leukemias, but also solid tumors, Aurorakinase A and B are interesting target structures for immunotherapeutic approaches.

Disclosure: No conflict of interest disclosed.

\section{P782}

Identification of acute myeloid leukemia-associated antigens recognized by allogeneic CD8+ T lymphocytes

\author{
Domning, S. 1; Köhler, S. 1; Distler, E. ${ }^{1}$; Pohl, C. ${ }^{2}$; Lennerz, V. ${ }^{1}$; \\ Herr, W. ${ }^{1}$; Wölfel, T. ${ }^{1}$; Wölfel, C. ${ }^{1}$
}

${ }^{1}$ III.Med.Klinik, Universitätsmedizin der Johannes Gutenberg-Universität, Mainz, Germany, ${ }^{2}$ Institut für Pathologie, Universitätsmedizin der Johannes Gutenberg-Universität, Mainz, Germany

CTL clones 1C6 and 4D7 against acute myeloid leukemia cells of patien MZ201 (AML FAB M5) were recently generated in allogeneic mixed lymphocyte leukemia cell cultures (allo-MLLC) using CD8 ${ }^{+} \mathrm{CD} 62 \mathrm{~L}^{\text {high }}+$ cells isolated from unrelated HLA class I-matched donor 332 (Distler, E., et al., 2008). Both CTL clones were used for T cell-directed expression screening of a cDNA library constructed from MZ201-AML cells as previously described (Wölfel, C., et al., 2008).

Library screening with 4D7 led to the identification of a novel minor histocompatibility antigen, PLAUR-317p, encoded by a known polymorphic allele of the plasminogen activator urokinase receptor gene (PLAUR, syn.: uPAR, CD87; SNP rs4760) and restricted by HLA-B*56:01. Prolin $(\underline{P})$ on position 317 generated an aggretope. The 10 meric PLAUR-317 $\underline{P}_{316-325}$ peptide (SPTITLLMTA) was recognized at lowest concentrations (half maximal lysis at $5 \mathrm{nM}$ ) whereas the homologous PLAUR-317 $\underline{L}_{316-325}$ was not recognized. The frequency of PLAUR-317P was determined via LightCycler PCR. Seventyfour of 252 individuals $(29,4 \%)$ carried one PLAUR-317P allele and approximately $2 \%$ of individuals were homozygous for PLAUR-317P. PLAUR is known to be overexpressed in AML, especially in subtype FAB M5, in multiple myeloma and in various solid cancers.

As the target of 1C6 lymphocytes we identified chemokine (C-X-C motif) ligand 3 (CXCL3). Its immunogenic peptide (RLLRVALLL, amino acids 14-22) was recognized in association with HLA-A*02:01 and was derived from the signal sequence of CXCL3. The same peptide is also encoded by the related genes CXCL1 and CXCL2, both induce recognition by 1 C6 upon transfection. 1C6 recognized HLA-A*02:01+ leukemic cells of myeloid origin (AML and CML) (Distler, E., et al., 2008). Monocytes and endothelial cells, but not epithelial cells, isolated from HLA-A*02:01+ donors were weakly recognized. Their recognition could be strongly enhanced by pretreatment with proinflammatory molecules (IL-1ß, TNF- $\alpha$ and LPS).

Using cDNA expression cloning with allogeneic leukemia-reactive $\mathrm{T}$ cells generated from a healthy donor we identified two antigens of distinct categories, mHag and LAA, overexpressed in myeloid leukemias and, among nonmalignant cells, preferentially expressed in hematopoetic cells. Both antigens are candidates for GvL effects and GvHD.

Disclosure: No conflict of interest disclosed.

\section{P783}

\section{Pitfalls in transplantation of NOD/SCID interleukin-2 receptor gamma chain (NSG) -deficient mice with unsorted AML samples}

\author{
von Bonin, M. ${ }^{1}$; Bornhäuser, M. ${ }^{2}$; Waskow, C. ${ }^{3}$
}

${ }^{1}$ SFB655 / CRTD / Medizinische Klinik und Poliklinik I, AG Waskow, Dresden, Germany, ${ }^{2}$ Medizinische Klinik und Poliklik I, Universitätsklinikum Dresden, Dresden, Germany, ${ }^{3}$ CRTD / SFB655, Dresden, Germany

Animal models have become indispensable in the exploration of malignant human tissues. Using non-obese diabetic/severe combined immunodeficient (NOD/SCID) mice, a hierarchy tree has been proposed for Acute Myeloid Leukemia (AML). However, only a minority of AML samples engraft into NOD/SCID recipients - even under optimal conditions. To improve engraftment frequency, more stringently immunocompromised mouse models carrying null mutations in the interleukin-2 receptor gamma chain gene ( $\left.I l 2 \mathrm{rg}^{\mathrm{y/}}\right)$ have been introduced. Here we compare NOD/SCID $I l 2 \mathrm{rg}^{\mathrm{y/}}$ (NSG) and $\mathrm{BALB} / \mathrm{c} \mathrm{Rag} 2^{-/-} \mathrm{Il} 2 \mathrm{rg}^{\mathrm{y} /-}$ recipient mice in their capacity to support engraftment of unsorted AML samples. Primary AML cells failed to engraft in unirradiated BALB/c Rag2 ${ }^{-/} I l 2 r g{ }^{y /-}$ mice, and only one AML sample engrafted successfully in sublethally irradiated animals as determined by $63 \%$ bone marrow (BM) chimerism. Yet, this AML sample showed an aggressive and refractory course in the clinic. In NSG mice, even in unirradiated recipients, engraftment of human (h) $\mathrm{CD} 45^{+}$cells was observed in $60 \%$ of mice, when transplanting $\geq 10^{7}$ cells, independent of the AML subtype or blast percentage $(11-87 \%$, $\mathrm{n}=6$ ). The majority of engrafted $\mathrm{hCD} 45^{+}$cells in the peripheral blood (PB) expressed CD3 (41-99\% of hCD45 cells, n=13), CD4 (65\%) and TCRa/b chains $(97 \%)$. In one experiment a seperate $\mathrm{CD} 19^{+}$population was additionally observed $\left(13 \%\right.$ of $\mathrm{hCD}^{+} 5^{+}$in $\left.\mathrm{PB}\right)$, but we consistently failed to obtain myeloid engraftment in the PB. None of the engrafted NSG mice showed thymus enlargement compared to untreated controls suggesting the absence of de novo $\mathrm{T}$ cell development and expansion of transferred donor $\mathrm{CD}^{+}$cells. We conclude, that NSG recipients support the engraftment by expansion of cells of the lymphoid lineage but they are refractory to the engraftment of myeloid cells in this experimental setting.

Disclosure: No conflict of interest disclosed.

\section{P784}

IDH mutations in therapy-related and secondary AML evolving from myelodysplastic syndromes

\section{Bodner, C. ${ }^{1}$; Pichler, M.M. ${ }^{1}$; Deutsch, A.J. ${ }^{1} ;$ Beham-Schmid, C. ${ }^{2}$;} Linkesch, W. ${ }^{1}$; Sill, H. $^{1}$; Wölfler, A. ${ }^{1}$

${ }^{1}$ University Hospital, Department of Hematology, Graz, Austria, ${ }^{2}$ University Hospital, Department of Pathology, Graz, Austria

Mutations in the NADPH-dependent isocitrate dehydrogenase 1 and 2 genes have recently been identified in de novo acute myeloid leukemia (AML) and in patients with leukemic transformation of JAK 2 mutation positive myeloproliferative disease. However, the pathogenetic impact and frequencies of these mutations in patients with other subtypes of AML, such as therapy-related AML (t-AML) or secondary AML (sAML) evolved from myelodysplastic syndromes (MDS) are not known. We therefore did a mutational analysis of the affected arginine residues in the IDH1 and IDH2 genes in 101 patients suffering from either t- or sAML by direct DNA sequencing. We identified one IDH1 (R132H) and three IDH2 mutations (2xR140Q and R172K) in 74 patients with t-AML resulting in a frequency of $1.4 \%$ and $4.1 \%$ mutations in IDH1 and IDH2, respectively. The frequencies of mutated IDH1 and IDH2 found in our t-AML cohort were lower as compared to the reported percentages in de novo AML (1.4\% vs. $8.5 \%^{1}, \mathrm{p}<0.05$; and $4.1 \%$ vs. $15.4 \%^{2}, \mathrm{p}<$ $0.05)$. Since IDH mutations are associated with a normal karyotype in de novo AML, the high prevalence of cytogenetic aberrations in t-AML may explain the lower frequencies of IDH1/2 mutations in t-AML patients. In 27 patients with sAML, we found three mutations in IDH2 (11.1\%; R140Q and 2x $\mathrm{R} 172 \mathrm{~K}$ ) but none in IDH1. All three IDH2 mutations were detectable in bone marrow cells obtained at the time of MDS diagnosis demonstrating acquisition of IDH2 mutations already during the myelodysplastic phase of the disease. All identified mutations in SAML and t-AML at diagnosis were heterozygous. However, one of the sAML patients showed loss of the wildtype IDH2 allele 
in a relapse sample after induction chemotherapy and autologous stem cell transplantation. SNP array-based analysis revealed copy-neutral loss of heterozygosity of $15 \mathrm{q} 25.1$ to 15 qter in the relapse sample implicating uniparental disomy of the region carrying the mutated IDH2 allele. In summary, while IDH mutations are less common in t-AML, mutated IDH2 can be found in a substantial fraction of patients with sAML already during the myelodysplastic phase of the disease. Uniparental disomy of $15 q$ was found as a late event in leukemic evolution of such a patient resulting in homozygosity of mutated IDH2.

${ }^{1}$ Mardis et al. N Engl J Med. 2009;361(11):1058-1066

${ }^{2}$ Ward et al. Cancer Cell 2010;17(3):225-234

Disclosure: No conflict of interest disclosed.

\section{P785 \\ Monitoring of minimal residual disease by $\mathrm{q}-\mathrm{PCR}$ in $\mathrm{AML}$ patients carrying NPM1 mutations}

Blau, O. ${ }^{1}$; Sindram, A. ${ }^{1}$; Burmeister, T. ${ }^{1}$; Graul, K. ${ }^{1}$; Seyde, C. ${ }^{1}$; Thiel, E. ${ }^{1}$; Blau, I.W. ${ }^{1}$

${ }^{1}$ Charite, Universitätsmedizin Berlin, Campus Benjamin Franklin, Klinik für Hämatologie, Onkologie und Transfusionsmedizin, Berlin, Germany

Mutations within the nucleoplasmin-1 gene (NPM1) occur in about 55\% of AML with normal karyotype and represent the single most frequent molecular aberration in AML patients. The mutations usually occur at exon 12 and induce most frequently an insertion of four base pairs. Currently, more than 40 different mutations can be identified. The most common subtypes of mutation, type A, B, and D, consisting of the duplication of the four bases TCTG, insertion of CATG or CCTG tetra nucleotide, respectively, represent about $90 \%$ of NPM1-positive AML. NPM1 mutations, with their high frequency in AML represent suitable MRD markers in both patients after chemotherapy and in the patients after allogeneic stem cell transplantation. Monitoring of minimal residual disease (MRD) is definitely of great value in clinical decision making. We describe a novel sensitive, rapid and reproducible method for detecting MRD in AML with NPM1-A, -B, and -D mutations with ROTORGENE system RQ-PCR. As positive control and for standard dilution curves, we used cell line OCI-AML for NPM1 type A and patient-specific dilution series of type $\mathrm{B}$ and $\mathrm{D}$ to reach the goal of a reproducible sensitivity of $10^{-4}$. Furthermore, the sensitivity of the assay was tested in a serial dilution of plasmids with types A and B mutations. NPM1 gene mutations were identified in 26 AML patients at the time of diagnosis. Mutation A was found in 13 patients $(50 \%)$, variant $\mathrm{B}$ in $6(23 \%)$, and variant $\mathrm{D}$ in 4 cases $(15 \%)$. At median, 5 qPCR analyses were performed for every patient. Median follow-up are 310 days; range, 5-786 days. Quantitative assessment of NPM1mut seems to provide a reliable MRD marker after chemotherapy and in the post transplantation period, predicting relapse.

Disclosure: No conflict of interest disclosed.

\section{P786}

\section{Expression of TGF- $\beta$ receptor ALK- 5 has a negative impact on overall survival of patients with Acute Myeloid Leukemia}

Otten, J. ${ }^{1}$; Schmitz, L. ${ }^{1}$; Vettorazzi, E. ${ }^{1}$; Schultze, A. ${ }^{1}$; Krauter, J. ${ }^{2}$; Loges, S. ${ }^{1}$; Bokemeyer, C. ${ }^{1}$; Fiedler, W. ${ }^{1}$

${ }^{1}$ Universitätsklinikum Hamburg-Eppendorf, Hamburg, Germany, ${ }^{2}$ Hannover Medical School, Hannover, Germany

Introduction: TGF- $\beta$ signalling is involved in angiogenesis with type I receptor ALK-1 mainly found on endothelial cells and ALK-5 on various cell types including endothelial and tumor cells. Since bone marrow neoangiogenesis plays an important role in acute myeloid leukaemia (AML), the TGF- $\beta$ pathway might be involved in the pathogenesis of AML.

Methods: Pretherapeutic samples from 93 AML patients enrolled into the AMLSG 07-04 study group (Austrio-German AMLSG study group) were studied for expression of receptors ALK-1 and ALK-5, their resprective ligands TGF- $\beta 1$ and bone morphogenic proteins (BMP) 9 and 10. In order to evaluate receptor activation, expression of downstream targets ID-1 and PAI-1 was analyzed. Uni- and multivariate analyses were performed using clinical and gene expression variables.

Results: ALK-1 and ALK-5 expression was found in the majority of AML patients (82\% and $88 \%$, respectively). The percentage of BMP-expressing patients was low (8\% for BMP-9, 13\% for BMP-10), whereas all patients expressed TGF- $\beta 1$. Expression of downstream target genes ID-1 and PAI-1 was found in almost all patients ( $99 \%$ and $100 \%$, respectively).

We analyzed the impact of gene expression on achievement of CR and eventfree (EFS), relapse-free (RFS) and overall survival (OS). Patients with low vs. high ALK-5 expression achieved a CR rate $63 \%$ vs. $27 \%$, respectively. Multivariate analysis included ALK-5, TGF- $\beta 1$ and ID- 1 gene expression as well as the baseline variables sex, age, karyotype and FLT3 mutation status. During the stepwise procedure of the Cox regression, several variables were eliminated. Only ALK-5 and the karyotype showed a significant impact on event-free, relapse-free and overall survival (ALK-5: $\mathrm{p}=0.001$ for EFS, $\mathrm{p}<$ 0.001 for RFS, $\mathrm{p}=0.008$ for OS; karyotype: $\mathrm{p}<0.001$ for EFS, $\mathrm{p}<0.001$ for RFS, $\mathrm{p}=0.008$ for OS).

Conclusions: In the majority of AML patients, ALK-1 and ALK-5 expression was found. Since downstream target gene expression was shown, both receptors were in an activated state. High ALK-5 expression resulted in a low CR rate which translated into reduced EFS, RFS and OS of AML patients. Therefore ALK-5 expression represents a new prognostic marker in AML. Since ALK-1 and ALK-5 are proposed to be involved in the regulation of vascular endothelial growth factor which is believed to the central growth factor in angiogenenis (Shao, Blood. 2009 Sep 3;114(10):2197-206.), the ALK-1/ ALK-5 pathway might represent a novel therapeutic target in AML.

Disclosure: No conflict of interest disclosed.

\section{P787}

\section{High aldehyde dehydrogenase activity characterizes a stem cell population without aberrant marker expression in Acute Myeloid Leukemia (AML)}

\author{
Hoang, V.T. ${ }^{1}$ Ran, D. ${ }^{1}$; Eckstein, V. ${ }^{1}$; Taubert, I. ${ }^{1}$; Jauch, A. ${ }^{2}$; \\ Ho, A.D. ${ }^{1}$
}

${ }^{1}$ Universitätsklinikum Heidelberg, Innere Medizin V, Heidelberg, Germany, ${ }^{2}$ Universität Heidelberg, Institut für Humangenetik, Heidelberg, Germany

Introduction: In $\mathrm{CD} 34^{+} \mathrm{AML}$, the leukemic stem cells are thought to reside within the $\mathrm{CD} 34^{+} \mathrm{CD} 38^{-}$cell compartment. However, in CD34- $\mathrm{AML}$ stem cells can be found in both $\mathrm{CD} 34^{+}$and CD34- population. Since CD34 expression is highly variable and not always correlated with stem cell feature, we used aldehyde dehydrogenase (ALDH) that is highly active in stem cells of normal bone marrow and cord blood for identification of their counterpart in AML. Indeed, $\mathrm{SSC}^{\mathrm{low}} \mathrm{ALDH}{ }^{\text {bright }}\left(\mathrm{ALDH}^{\mathrm{br}}\right)$ cells contained increased number of LTC-IC and could engraft in NOD/SCID mice. In this study we want to specify the leukemic versus normal hematopoietic stem cell characteristics for the $\mathrm{ALDH}^{\mathrm{br}}$ stem cell population.

Methods: We determined the expression of lineage aberrant markers/marker combinations (e.g. CD2, CD7, CD11b, CD15, CD19, CD22, CD56, $\mathrm{CD} 3^{+} \mathrm{CD} 33^{-}$and $\mathrm{CD}^{-} 3^{-} \mathrm{CD} 33^{+}$) on the $\mathrm{ALDH}^{\text {br }}$ versus the $\mathrm{CD} 34^{+} \mathrm{CD} 38^{-}$compartment in bone marrow samples from patients with newly diagnosed AML using multiparameter flow cytometry. Afterwards $\mathrm{ALDH}^{\mathrm{br}}$ and $\mathrm{ALDH}{ }^{\mathrm{br}} \mathrm{CD} 34^{+}$ cells were single cell sorted and cocultured with mesenchymal stromal cells acting as feeder layer. Colonies derived from single mother cells were analyzed by FISH and RT-PCR for genetic aberrations.

Results: In AML the frequency of $\mathrm{ALDH}^{\mathrm{br}}$ cells was $0.56 \%$ (range: 0.02 $3.67 \%$ ) of mononucleated cells, much lower than the amount of CD34+ cells (median: $23 \%$, range: $0.12-56.5 \%$ ). A high CD34 expression was observed on $65.4 \%$ (range: $4.1-100.0 \%$ ) of the $\mathrm{ALDH}^{\mathrm{br}}$ subset. In comparison, mononucleated cells from healthy bone marrow contained $1.23 \%$ (range: 0.60 $1.99 \%$ ) $\mathrm{ALDH}^{\mathrm{br}}$ cells, of which $76.2 \%$ (range: $67.2-86.8 \%$ ) were CD34 ${ }^{+}$. Interestingly, more than $90 \%$ of $\mathrm{ALDH}^{\text {br }}$ cells were free of aberrant markers, which were largely expressed on the $\mathrm{CD} 34^{+} \mathrm{CD} 38^{-}$compartment as well as on whole blasts. The malignant expression of these markers on leukemic blasts were confirmed by flow cytometry, since they were absent or present in a very low frequency within the $\mathrm{CD} 34^{+} \mathrm{CD} 38^{-}$cells in healthy samples.

Conclusion: Although both ALDH activity and CD34 and CD38 surface markers have been used to identify stem cells in AML, we could show that the $\mathrm{ALDH}^{\mathrm{br}}$ and $\mathrm{CD}^{+} 4^{+} \mathrm{CD} 38^{-}$populations can be distinguished from each other 
by the expression of aberrant markers. Further cytogenetic and molecular characterizations of these populations might be helpful to verify their leukemic versus normal origins.

Disclosure: No conflict of interest disclosed.

\section{P788}

\section{Expression of NK cell receptors on blast cells of CD56- positive acute myeloid leukemias}

\author{
Fuhrmann, S. ${ }^{1}$; Schabath, R. ${ }^{1}$; Karawajew, L. ${ }^{2}$; Ludwig, W.-D. ${ }^{1}$; \\ Ratei, R. ${ }^{1}$ \\ ${ }^{1} \mathrm{HELIOS}$ Klinikum Berlin-Buch, Klinik für Hämatologie, Onkologie und \\ Tumorimmunologie, Berlin, Germany, ${ }^{2}$ Charite Medical School, \\ Experimental and Clinical Research Center, Berlin, Germany
}

Background: A subset of acute myeloid leukemias is characterized by the coexpression of CD56, a canonical natural killer (NK) cell marker. Whether additional NK specific markers are expressed is not known.

Aims: Detailed immunophenotypic characterization of CD56 positive AMLs in order to detect multiple NK- cell specific markers compatible with a NK-cell lineage derivation.

Methods: We used nine color flow-cytometry to analyse the expression of NK-cell specific markers on CD56 positive acute myeloid leukemic blasts. Bone marrow aspirates of patients with newly diagnosed CD56 positive AML were characterized for their expression of CD94, CD122, CD161, CD244 and CD314. These samples were also analyzed in detail for their expression of myeloid (CD13, CD33, CD65, CD15, MPO, LF), monocytic (CD14, CD64, CD4), B-lymphoid (CD19, CD20, CD22, CD79a), T-lymphoid (CD3, CD2, $\mathrm{CD} 5, \mathrm{CD} 7, \mathrm{CD} 4, \mathrm{CD} 8)$ and progenitor (CD34, CD133, TdT, CD117) markers.

Results: 41 patients (18 female/ 23 male) with a mean age of 48 years were analyzed. Blast cells from all patients were negative for $\mathrm{CD} 3$ and positive for CD13 and/or CD33. The percentage of CD56-positive blasts varied between $21 \%$ and $97 \%$ with a mean of $58 \%$ for the whole group. 39 patients $(95 \%)$ were positive for at least one of the NK markers and this was irrespective of FAB morphology. The mean percentage of expression for CD94, CD122, CD161, CD244 and CD314 on the blast cell population was 12,4\%, 20,7\%, $60,9 \%$ and $7,2 \%$, respectively. NK markers were detected both, on CD56 positive and CD56 negative blast cells, but there was a trend towards higher expression of CD122 and CD244 on CD56 positive blasts (adjusted p=0,067 and $\mathrm{p}=0,089$, respectively).

Conclusion: Expression of NK cell receptors is demonstrated in more than 90\% of CD56 positive AML but can also be detected on CD56 negative blast cells. CD161 is the most frequently detected NK cell receptor in CD56 positive AML. The biological meaning and the clinical impact of NK cell receptor expression on myeloid blast cells has to be investigated in larger trials.

Disclosure: No conflict of interest disclosed.

\section{P789}

\section{Clinical relevance of NPM1 and FLT3 mutations in AML patients}

\section{Blau, O. ${ }^{1}$; Baldus, C.D. ${ }^{1}$; Graul, K. ${ }^{1}$; Sindram, A. ${ }^{1}$; Thiel, E. ${ }^{1}$;} Blau, I.W. ${ }^{1}$

${ }^{1}$ Charite, Universitätsmedizin Berlin, Campus Benjamin Franklin, Klinik für Hämatologie, Onkologie und Transfusionsmedizin, Berlin, Germany

Acute myeloid leukemia (AML) is a clonal disorder affecting pluripotent stem cell and characterized by ineffective hematopoiesis. Most AML patients harbor cytogenetic and molecular defects that identify entities with peculiar biological and clinical data and distinct therapeutic responses. Overall, about $50-60 \%$ of de novo AML and $80-95 \%$ of secondary AML patients display chromosomal aberrations. A growing number of evidence suggests that mutations of multiple genes mediate the pathogenesis and progression of AML. Molecular analysis reveals recurrent genetic markers in more than $85 \%$ of AML patients with normal karyotype. These genetically based subtypes are associated with diverse biological characteristics and distinct clinical profiles. Mutations of the FMS-like tyrosine kinase-3 (FLT3) gene, including both point mutations within the tyrosine kinase domain (TKD) and internal tandem duplication (ITD) in the juxtamembrane domain, are the most common genetic changes seen in AML. The FLT3-ITD, with a 35\%-45\% frequency in normal karyotype AML, has an adverse impact on prognosis. Fewer incidences are the FLT3-TKD mutations which are represented by single nucleotide base exchanges. AML carrying the nucleoplasmin-1 gene (NPM1) mutation causing aberrant cytoplasmic expression of nucleoplasmin account for approximately one-third of adult AML, but 55\% of cases with normal karyotype.

In the present study we analyzed the prevalence of NPM1 and FLT3 mutations and its association with hematological and cytogenetic data, complete remission (CR) rate, and overall survival (OS) in 125 patients newly diagnosed with AML. Median age was 55 years, range 25-77. NPM1 gene mutations were identified in $26(21 \%)$ patients, FLT3-ITD in 26 (21\%) patients, and FLT3TKD in $7(6 \%)$ patients. In AML patients with normal karyotype, the frequencies of the both mutations were significantly higher than in those without. Both FLT3 and NPM1 mutations were related to high peripheral white blood cell counts. Compared with NPM1(-)AML patients, NPM1(+)AML patients were associated with higher CR rate after induction therapy and better OS. FLT3-ITD(+)AML patients compared with FLT3-ITD(-)AML patients were associated with lower CR rate and worse OS. We conclude that NPM1 and FLT3-ITD mutations are common in AML patients with normal karyotype and related with the clinical characteristics and prognosis of AML.

Disclosure: No conflict of interest disclosed.

\section{P790}

\section{Jurkat cells as a model to study the SDF-1/CXCR4 axis}

\section{Zepeda-Moreno, A. ${ }^{1}$; Wagner, W. ${ }^{2}$; Taubert, I. ${ }^{1}$; Saffrich, R. ${ }^{1}$;} Bork, S. ${ }^{1}$; Horn, P. ${ }^{1}$; Eckstein, V. ${ }^{1}$; Ho, A.D.

${ }^{1}$ Universitätsklinikum Heidelberg, Innere Medizin V, Heidelberg, Germany, ${ }^{2}$ Institut für Biomedizinische Technologien, Stammzellbiologie und Cellular Engineering, Aachen, Germany

Introduction: As an important chemoattractant for hematopoietic stem cells, the chemokine SDF-1, may also support the survival and proliferation of leukemia cells, since myeloid and lymphoid leukemia cells express its receptor CXCR4 that allows their homing and retention in the bone marrow. In this context, we characterized several leukemia cell lines to understand the biology of the SDF-1/CXCR4 axis and find a model to simulate migration, proliferation, adhesion and observe different patterns of CXCR4 expression.

Methods: Jurkat, Kasumi, HL-60, KG1a and K562 cell lines were utilized for this study. Western blot (WB) and flow cytometry (FC) analyses were used to characterize the expression of CXCR4 and the adhesion molecules CD54, CD29 and CD34. Adhesion assays were made through our novel adhesion method using human mesenchymal stem cells as model for a bone marrow niche. Additionally, transwell migration and proliferation assays were performed.

Results: Western blot analyses showed that all cell lines expressed CXCR4, nevertheless FC demonstrated that Jurkat and Kasumi cells had a higher surface expression in comparison to HL-60, KG1a and $\mathrm{K} 562$ where this receptor could hardly be detected, but in which the same antigen was found intracellularly. Using FC and Jurkat cells, we measured the concentration and time dependent internalization of CXCR4 upon the stimulation with SDF-1 alpha, stimulation that made also possible to document CXCR4 degradation by WB. Since the affinity of the anti-CXCR4 12G5 antibody is lost when the SDF-1 competitor AMD3100 is occupying the receptor, we performed a fixation method to recover this binding, succeeding and recuperating the signal in the surface. Adhesion and migration studies showed that SDF-1 was able to induce mobilization in vitro, but not AMD3100 which could also inhibit it. No difference was seen in the pattern of expression of different surface adhesion molecules like CD54, CD29 and CD34 after the activation of the CXCR4 axis. Furthermore, after an incubation period of 5 days, AMD3100 and SDF-1 alpha led to a higher proliferation rate of Jurkat cells in comparison to untreated cells.

Conclusions: In this work we characterized several leukemia cells lines and the SDF-1/CXCR4 axis, finding that Jurkat cells are an excellent model to understand the biology of the SDF-1/CXCR4 axis since they simulate functionally the effects of the SDF-1/CXCR4 axis.

Disclosure: No conflict of interest disclosed. 
P791

\section{Leukemia-associated antigens are upregulated by the FIt-3 tyrosine kinase and recognized by AML-reactive T lymphocytes}

Brackertz, B. ${ }^{1}$; Conrad, H. ${ }^{1}$; Daniel, J. ${ }^{1}$; Kast, B. ${ }^{1}$; Peschel, C. ${ }^{1}$; Bernhard, $H^{1,2}$

${ }^{1}$ Klinikum rechts der Isar der TU München, München, Germany, ${ }^{2}$ Klinikum Darmstadt, Med. V, Darmstadt, Germany

Acute myeloid leukemia (AML) is the most common acute leukemia in adults. Mutations in the fms-like tyrosine kinase receptor 3 (Flt3) occur in approximately $30 \%$ of AML patients and are a bad prognostic factor. The two major types of mutations are internal tandem duplications (ITD) in the juxtamembrane domain and point mutations in the kinase domain (TKD). These mutations lead to constitutive activation of the Flt 3 kinase domain in the absence of Flt3 ligand (FL).

We analysed the influence of Flt3 on the expression of different leukemiaassociated antigens, such as Wt-1, Rhamm, PR-3, Survivin and Prame. First, we treated FLT3-WT ${ }^{+}$RS4-11 leukemia cells with increasing concentrations of FL and documented the expression of the leukemia-associated antigens. Second, we investigated the regulation of the leukemia-associated antigens in RS4-11 cells after electroporation with Flt3siRNA. Third, we cultured Flt3 ITD $^{+}$MV4-11 leukemia cells, which show high expression of the leukemiaassociated antigens Wt-1, Rhamm, PR-3, Survivin and Prame, with SU5416, a known inhibitor of the ITD-Flt-3 activity. The immunological goal of our research is the generation of human T cell clones against HLA-A2-binding peptides derived from Flt-3 regulated antigens. In order to establish high avidity $\mathrm{T}$ cells we stimulated HLA-A2- $\mathrm{CD}^{+}{ }^{+} \mathrm{T}$-lymphocytes from a healthy donor with autologous HLA-A2 ${ }^{+}$DCs loaded with the peptide of interest. After three stimulations peptide-specific $\mathrm{T}$ cells were sorted and cloned.

We could show that the treatment of RS4-11 leukemia cells with FL increased the expression of the leukemia-associated antigens Wt-1, Rhamm, PR-3, Survivin and Prame. Vice versa, the expression of these antigens was decreased following transduction of the RS4-11 cells with Flt3siRNA Analogue, the incubation of MV4-11 leukemia cells with the inhibitor SU5416 reduced the expression of Survivin, Wt-1, Rhamm, PR-3, and Prame. Furthermore, we have succeded to generate human $\mathrm{T}$ cell clones directed against Rhamm, PR-3, Survivin. Via ELISA, ELISpot and Chromium-Release assay we could show that our different CTL-clones recognized and lysed the HLA-A2 ${ }^{+}$leukemia cell line K562tA2 as well as HLA-A2 ${ }^{+}$AML blasts. In conclusion, the antigens Wt-1, Rhamm, PR-3, Survivin and Prame are upregulated by FLT-3 and, therefore, can serve as leukemia-associated target antigens for cytotoxic $\mathrm{T}$ lymphocytes. These results may have major implications on the future development of tailored therapies against AML.

Disclosure: No conflict of interest disclosed.

\section{P792}

\section{Heterogeneous interactions of leukemic and control bone marrow stroma cells with an AML cell line and AML leukemic blasts}

\section{Buttkereit, U. ${ }^{1,2}$; Mohamad, S. ${ }^{1}$; Lindemann, M. ${ }^{3}$; Göthert, J.R. ${ }^{1}$; Opalka, B. ${ }^{1}$; Dürig, J. ${ }^{1}$; Dührsen, U. ${ }^{1}$ \\ ${ }^{1}$ Uniklinik Essen, Klinik für Hämatologie, Essen, Germany, ${ }^{2}$ Uniklinik Essen, Klinik für Knochenmarktransplantation, Essen, Germany, ${ }^{3}$ Uniklinik Essen, Institut für Transfusionsmedizin, Essen, Germany}

Introduction: Tumor-stroma interaction plays a pivotal role for malignant cell survival, proliferation, immune escape, and drug resistance. We had previously shown that bone marrow (BM) stroma from leukemia patients had different gene expression patterns and support of normal CD34+ cells compared to controls (Ctr). Aim of this study was to evaluate growth, survival, and colonyforming potential issues of M-07e AML cells and AML blasts as indicator cells (IC) upon contact with non-leukemic and leukemic stroma.

Methods: Plastic-adherent BM cells from leukemic or Ctr non-leukemic donors were cultured for several weeks. Polyclonal as well as isolated single fibroblast colony (F-CFU) cultures were set up. Short-term and long-term cocultures of BM stroma used M-07e AML cells and AML blasts as IC. After long-term co-culture colony forming units (CFU) were determined in the adherent and non-adherent fraction of the IC. Proliferation of IC was determined by cell counting and $3 \mathrm{H}-\mathrm{TdR}$ incorporation assays. The human HS-5 stroma cell line was used in selected experiments.

Results: The F-CFU frequency was determined from 39 Ctr samples, 6 MDS samples, 59 leukemic samples, and 12 lymphoma samples with BM infiltration. Succession of F-CFU numbers in respective samples was AML / MDS < $\mathrm{Ctr}<\mathrm{MM} / \mathrm{FL} \mathrm{II}<<2^{\circ}$ AML. Polyclonal stroma cells from AML BM supported M-07e IC survival slightly but not significantly better than Ctr stroma. No noteworthy support for primary AML blasts was observed with either stroma. Stroma from both AML and Ctr donors stimulated colony formation of M-07e IC without marked differences in the frequency of compact and diffuse colonies. In 3H-TdR assays M-07e IC were stimulated by IL-3 and certain but not all stroma samples tested. Notably, IL-3-induced proliferation of $\mathrm{M}-07 \mathrm{e}$ IC was decreased in the presence of stroma and almost completely suppressed by HS-5 cells. When single F-CFU were isolated, expanded, and tested for their capacity to support M-07e IC stimulating as well as nonstimulating F-CFU were found. Support competence of stroma cells decreased with passage number.

Conclusion: Primary BM polyclonal stroma cells and single F-CFU from leukemic and non-leukemic donors showed a broad heterogeneity with respect to support of cell growth or colony-forming potential in favour of the AML M-07e IC line. Thus, the interaction of stroma and normal or leukemic hematopoietic cells seems to be a complex system awaiting further investigations.

Disclosure: No conflict of interest disclosed.

\section{Posterdiskussion}

ALL

\section{P793}

D,L-Methadone improves therapeutic efficiency of doxorubicin in treatment of leukemia cells and breaks chemo- and radioresistance

Friesen, C. ${ }^{1}$; Roscher, M. ${ }^{1}$; Hormann, I. ${ }^{1}$; Queudeville, M. ${ }^{2}$; Meyer, L.-H. ${ }^{2}$; Debatin, K.-M.' ${ }^{2}$ Miltner, E. ${ }^{1}$

${ }^{1}$ Institut für Rechtsmedizin, Universität UIm, Ulm, Germany, ${ }^{2}$ Klinik für Kinder- und Jugendmedizin, Universität UIm, Ulm, Germany

Aim: One of the primary causes for therapeutic failure in chemotherapy of leukemia and solid tumors is the resistance to chemotherapeutic drugs and/or radiation. Therefore, new options are needed to improve therapeutic success in the treatment of cancer. The therapeutic opioid drug methadone is a long-acting $\mu$-opioid receptor agonist and is a highly effective and safe medication for the opioid dependence of outpatients. In addition, methadone is of significant utility as a long-acting analgesic.

We examined in our studies the effect of D,L-methadone alone and D,Lmethadone in addition to doxorubicin on different $\mathrm{B}$ cell precursor (BCP)ALL cells in vitro and ex vivo.

Methods: Different leukemia cell lines and primary BCP-ALL cells isolated from xenografted NOD/SCID mice were treated with different therapeutic concentrations of D,L-methadone alone or in addition to therapeutic concentrations of doxorubicin. Cell death and apoptosis was measured by flowcytometry and activation of apoptosis pathways was analyzed by Western Blot analyses.

Results: We found that D,L-methadone sensitizes leukemia cells, expressing the opioid receptors, for doxorubicin treatment. At low therapeutic concentrations of D,L-methadone in combination with doxorubicin a strong increase of cell death was found in leukemia cell lines as well as in primary BCP-ALL cells. D,L-Methadone in addition to doxorubicin inhibited proliferation and induced cell death through apoptosis induction in leukemia cells at therapeutic concentrations. Activation of apoptosis pathways through activation of caspase- 3 and caspase-9, downregulation of Bcl- $\mathrm{x}_{\mathrm{L}}$ and XIAP, and cleavage of PARP were found after treatment with D,L-methadone in combination with doxorubicin in leukemia cells. Noteworthy, D,L-methadone reversed deficient induction of cell death and activation of caspases by doxorubicin in chemoand radioresistant leukemia cells.

Conclusions: These findings demonstrate that D,L-methadone improves therapeutic efficiency of doxorubicin in treatment of BCP-ALL leukemia cells in vitro and ex vivo and breaks chemo- and radioresistance in leukemia cells. 
This study provides the foundation for new strategies establishing methadone as additional therapeutic anticancer drug in leukemia therapy to improve therapeutic success.

Disclosure: No conflict of interest disclosed.

\section{P794 \\ Modulation of CXCR4 activity by CCL18 in pre-B acute lymphocytic leukemia cells}

\author{
Catusse, J. ${ }^{1}$; Wollner, S. ${ }^{1}$; Leick, M. ${ }^{1}$; Burger, M. ${ }^{1}$ \\ ${ }^{1}$ Uniklinik Freiburg, Hematologie/Onkologie, Freiburg, Germany
}

Introduction: Two homeostatic chemokines, CCL18 and CXCL12, have been shown to be present at high concentration in the serum of Acute Lymphocytic Leukemia (ALL). Chemokines are involved in the recruitment and localization of cells and particularly of immune cells in homeostasis and pathologica conditions. CXCL12 is centrally involved in the localization of B-cells at different stages of their differentiation; however, expression of its receptor CXCR4 does not correlate with the ability of CXCL12 to induce its response. CCL18 has been controversially reported to induce the classical chemokine responses (chemotaxis, intracellular calcium release, kinase phosphorylation) in different cell types, including B lymphocytes. Despite the extensive work carried out on CCL18, the identification of its receptor is still lacking. We have investigated the activity of these two chemokines on B-ALL cell activity. Methods: Expression of GPR30 at the surface of the pre-B ALL Nalm-6 cell line and of primary cells from pre-B ALL and common ALL patients was evaluated. These cells were then used to monitor CXCL12 induced intracellular calcium release as well as chemotaxis experiments in presence or not of CCL18 and neutralizing GPR-30 antibody. Transfected cells have been used to ensure the involvement of GPR30 in these observations.

Results: We showed that GPR30, a chemokine-like receptor, is expressed at the surface of pre-B ALL cells but not at the surface of common ALL primary cells. In addition, we could demonstrate GPR30-dependent binding of CCL18 to cells. Furthermore, CCL18 binding to GPR30 can modify the responses of another chemokine receptor: CXCR4, the receptor for the chemokine CXCL12 (formerly called SDF-1) centrally involved in B cell maturation. Activation of CXCR4 by CXCL12 was reduced in presence of CCL18 when tested in calcium mobilization and chemotaxis assays.

Conclusions: Regulation of CXCR4 activation throughout B cell differentiation has been shown to be finely controlled. We identified here CCL18 as being a GPR30 ligand regulating CXCR4 thus showing control of CXCL12 induced cellular activation. This is to be related to CCL18 and CXCL12 high level concentration in serum of ALL patients. Hence, we suggest CCL18 to be involved in the localization and maturation of pre-B ALL with some impact in the pathophysiology of pre-B ALL.

Disclosure: No conflict of interest disclosed.

\section{P795}

\section{Expression of the CDK-inhibitor P26 ${ }^{\text {INCA1 }}$ in acute Iymphoblastic leukemia}

Lohmeyer, L. ${ }^{1}$; Tschanter, P.1,2; Bäumer, N. ${ }^{1}$; Berkenfeld, F.1; Stelljes, M. ${ }^{1}$; Brüggemann, M. ${ }^{3}$; Kneba, M. ${ }^{3}$; Berdel, W.E. ${ }^{1}$; Koschmieder, S. ${ }^{1,2}$; Müller-Tidow, $C^{1,2}$

${ }^{1}$ Department of Medicine A Hematology, Oncology and Pneumology, University Münster, Münster, Germany, ${ }^{2}$ Universität Münster, IZKF, Münster, Germany, ${ }^{3}$ 2nd Medical Department, University Hospital Schleswig-Holstein, Kiel, Germany

Acute lymphoblastic leukemia (ALL) results from malignant transformation of lymphoid progenitor cells. CDK inhibitors such as p16INK4A play an important role in ALL pathogenesis. The newly discovered CDK inhibitor p26INCA1 is consistently expressed in B-cells. Here, we analyzed expression of INCA1 mRNA in ALL. To evaluate the concept that altered levels of Incal, its interaction partners, and further cell cycle inhibitors contribute to unrestricted proliferative activity in leukemic blasts we analysed mRNA levels of 61 acute lymphoid leukaemia (ALL) patients. By using high-throughput quantitative RT-PCR we compared respective gene expression levels in bone mar- row aspirates derived from patients with pro-B-ALL $(n=4)$, common-B-ALL $(n=23)$, pre-B-ALL $(n=8), B-A L L ~(n=10)$ and T-ALL $(n=16)$ with 10 control samples containing normal B- and T-cells. In all leukemia subgroups Incal mRNA levels were found to be significantly decreased compared to controls. Gene expression analysis of the Incal interaction partners cyclin A1 and cyclin A2 as well as expression levels of other cell cycle inhibitors such as $\mathrm{p} 16^{\text {Ink4a }}, \mathrm{p} 21^{\text {Cip1/Waf1 }}$, and $\mathrm{p} 27^{\text {Kip } 1}$ revealed a heterogeneous pattern of gene expression levels in different ALL subtypes. To evaluate the hypothesis that Incal expression could be relevant for the prognosis of ALL, we compared Incal-mRNA expression levels in patients with low, standard and high risk as well as in standard risk patients with and without relapse. Incal was found to be expressed at lower levels in the high-risk-group compared to the standardrisk-group and in relapsing compared to non-relapsing patients. Our data suggest that low expression of Incal and other cell cycle inhibitors may be associated with the proliferative capacity of leukemic blasts. The role of INCA1 as a novel biomarker for ALL prognosis warrants further investigation.

Disclosure: No conflict of interest disclosed.

\section{P796}

\section{Concomitant treatment of Sorafenib with dexamethasone enhances antiproliferative effects in lymphoblastic cells}

\section{Schult, C. ${ }^{1}$; Fischer, K. ${ }^{1}$; Dahlhaus, M. ${ }^{1}$; Goetz, M. ${ }^{1}$; Lange, S. ${ }^{1}$;} Freund, M. ${ }^{1}$; Junghanss, C. ${ }^{1}$

${ }^{1}$ Universität Rostock, Hämatologie/Onkologie/Palliativ Medizin, Rostock, Germany

Introduction: Sorafenib is a multikinase inhibitor targeting Raf serine/threonine as well as different receptor tyrosine kinases. Sorafenib has been approved for the treatment of renal cell carcinoma and it is under clinical investigation in FLT3 positive acute myeloid leukemia patients. In a previous study, we could demonstrate that Sorafenib inhibits cell proliferation and induces apoptosis as well as necrosis by inhibiting PI3K/Akt signalling in acute lymphoblastic leukemia (ALL) cells. Here, we examined whether combination of Sorafenib with cytotoxic agents might enhance antiproliferative effects in ALL cells in vitro.

Methods: B- and T-ALL cell lines (SEM, Jurkat and MOLT4) with different cytogenetic background were investigated. Cells were treated with Sorafenib $(3.65 \mu \mathrm{M})$ in combination with sub IC 50 concentrations of cytarabine $(0.125$ $\mu \mathrm{M}$ or $0.05 \mu \mathrm{M})$, doxorubicin $(25 \mathrm{nM})$ or dexamethasone $(1 \mu \mathrm{M}$ or $10 \mu \mathrm{M})$ for up to $72 \mathrm{~h}$. Cell count, apoptosis rates, necrosis rates and metabolic activity were determined by microscopy, flow cytometry and WST-1 testing at $24 \mathrm{~h}$, $48 \mathrm{~h}$ and $72 \mathrm{~h}$, respectively.

Results: Single treatment with Sorafenib at $3.65 \mu \mathrm{M}$ inhibited the proliferation of all ALL cell lines significantly. Sorafenib induced antiproliferative, apoptosis and necrosis effects in vitro. Concomitant treatment of the cells with cytarabine or doxorubicin in addition to Sorafenib did not enhance the inhibition of proliferation. Additive effects in regards to an increase of apoptosis or necrosis rates were not detected. However, concomitant treatment with dexamethasone led to enhanced inhibition of the cell proliferation and induced an increase of necrosis rates in SEM cells. Further, metabolic activity decreased after $72 \mathrm{~h}$ when Sorafenib and dexamethasone were combined $(30.4 \%)$ in contrast to $3.75 \mu \mathrm{M}$ Sorafenib $(45.5 \%)$ or $1 \mu \mathrm{M}$ dexamethasone $(89.4 \%)$ alone compared to DMSO $(100 \%)$ treated cells.

Conclusions: Concomitant treatment of ALL cells with cytarabine or doxorubicin in addition to Sorafenib does not result in synergistic cytotoxic effects in vitro. However, dexamethasone might have synergistic effects when given concomitant to the multikinase inhibitor.

Disclosure: Catrin Schult: No conflict of interest disclosed. Christian Junghanss: Financing of Scientific Research: Wissenschaftliche Untersuchungen wurden von BayerHealthcare, Leverkusen finanziert. 
P797

\section{Leukemias induced by altered TRK-signaling are sensitive to $\mathrm{mTOR}$ inhibitors in preclinical models}

Rhein, M. ${ }^{1}$; Schwarzer, A. ${ }^{1}$; Yang, M. ${ }^{1}$; Kaever, V. ${ }^{2}$; Brugman, M. ${ }^{1}$ Meyer, J. ${ }^{1}$; Ganser, A. ${ }^{3}$; Baum, C. ${ }^{1} ;$ Li, Z. ${ }^{1}$

${ }^{1}$ Hannover Medical School, OE6960, Experimentelle Hämatologie, Hannover, Germany, ${ }^{2}$ Hannover Medical School, Pharmakologie,

Hannover, Germany, ${ }^{3}$ Hannover Medical School, OE6860, Klinik für Hämatologie, Hämostaseologie, Onkologie und Stammzelltransplantation, Hannover, Germany

Rapamycin is a potent allosteric mTORC1 inhibitor with clinical applications as an anti-cancer agent. However, only a fraction of cancer patients responds to the drug, and no biomarkers are available to predict tumor sensitivity. Recently, we and others have obtained evidence for potential involvement of TRK (tropomyosin-related kinase) receptor protein tyrosine kinases (TRKA TRKB, TRKC) in leukemia. In the present study, we tested the therapeutic effect of Rapamycin and its analog RAD001 on altered TRK-induced lymphoblastic leukemia in a murine model. Daily treatment with Rapamycin $(2 \mathrm{mg} /$ $\mathrm{kg})$ or RAD001 $(1 \mathrm{mg} / \mathrm{kg})$ significantly prolonged the survival of treated animals $(n=34)$ compared with the placebo group. Consistently, both mTOR and S6 proteins were strongly dephosphorylated in vitro and in vivo after treatment with Rapamycin or RAD001. However, Rapamycin did not completely inhibit mTORC1-dependent phosphorylation of 4E-BP1, and no activation of MAPK or Akt pathways was observed in resistant tumors. Interestingly, leukemic cells isolated from a Rapamycin-resistant mouse were still highly sensitive to Rapamycin in vitro. Our findings suggest that altered TRK signaling may be a good predictor of tumor sensitivity to mTOR inhibition, and that pathways other than MAPK and Akt exist that may trigger resistance of leukemic cells to Rapamycin in vivo.

Disclosure: No conflict of interest disclosed.

\section{P798}

\section{Downregulation of Interleukin-1 gene expression after application of the antiemetic agent ondansetron to acute lymphoblastic leukemia cells in vitro}

Prada, J. ${ }^{1}$

${ }^{1}$ Charité - CVK, Forschungshaus, Berlin, Germany

Introduction: Ondansetron is an antiemetic agent which is widely used in the prophylactic treatment of chemotherapy-induced nausea and vomiting (CINV) in children with acute lymphoblastic leukemia (ALL). It has previously been observed that ondansetron is able to inhibit proliferation of ALL cells in vitro, by activation of a pathway which involves the activation of the inducible NO-synthase (iNOS).

Methods: The expression of representative inflammatory cytokines, such as interferon-gamma (IFN-gamma) and interleukin-1 (IL-1), was analysed after application of ondansetron to ALL cells in vitro. For this purpose, we used the B-cell precursor leukemia cell line REH derived from a patient with ALL. The concentrations of IFN-gamma in cell supernatants were analysed by using a commercially available ELISA kit, and the gene expression of IL-1 was evaluated by systematic mRNA dot blot hybridizations performed with aid of a specific gene probe for human IL-1.

Results: When ondansetron was applied at concentrations of 5-50 $\mu \mathrm{M}$ to cultured REH cells, the expression of IFN-gamma was found to be significantly increased (up to $300 \%$ ), after $72 \mathrm{~h}$ incubation in standard conditions. Contrastingly, the corresponding gene expression of IL-1 was significantly reduced (up to 50\%) after the application of ondansetron at the mentioned

Table 1. Days of delay at the beginning of each cycle (for Abstract P799)

\begin{tabular}{|l|l|l|l|l|l|l|l|l|}
\hline & Ind 1/Ind 2 & Kons 1 & Kons 2 & Reind 1 & Reind 2 & Kons 3 & Kons 4 & Kons 5 \\
\hline Median & $0 / 14$ & 40 & 49 & 71 & 81 & 101 & 118 & 139 \\
\hline Maximum & $9 / 50$ & 80 & 75 & 88 & 87 & 115 & 143 & 136 \\
\hline Minimum & $0 / 0$ & 3 & -2 & 6 & 41 & 50 & 55 & 197 \\
\hline
\end{tabular}

standard concentrations of $5-50 \mu \mathrm{M}$ and further $72 \mathrm{~h}$ incubation in similar conditions.

Conclusions: The presented results indicate that the antiemetic agent ondansetron is able to differently modulate the expression of inflammatory cytokines such as IFN-gamma and IL-1, simultaneously to an extended iNOS upregulation and an inhibition of cell proliferation. These results represent additional beneficial effects of ondansetron and further support its use in the treatment of CINV in patients with ALL.

Disclosure: No conflict of interest disclosed.

\section{P799} with the protocol of the GMALL study of 07/03 in a single institution

Lässer, C. ${ }^{1}$; Sperr, W.R. ${ }^{1}$; Fonatsch, C. ${ }^{2}$; Knöbl, . $^{1}$; Streubel, B. ${ }^{3}$; Jäger, U. ${ }^{1}$; Heinz, R.

edical University Vienna, Dept. of Medicine I, Division of Hematology and Hemostaseology, Vienna, Austria, ${ }^{2}$ Medical University of Vienna, Vienna, Clinical Institute of Pathology, Vienna, Austria, ${ }^{4}$ Medical University Vienna, Dept. of Medicine I, Bone Marrow Transplantation Unit, Vienna, ospital Schleswig-Holstein, Kiel, Germany, ${ }^{6}$ Medical University Vienna, Clinical Institute of Medical and Chemical Laboratory Diagnostics, Vienna, Austria

Introduction: Multimodal strategies, like the protocols of the GMALL focus on risk adapted therapies for ALL with strict dosage and time intervals. Although remission rates increased, long term survival is still low in adults. This may be due to the lack of feasibility of the protocols. Surprisingly, information on the impact of treatment delay and dose reduction as well as the feasibility of ALL protocols is rare.

Patients and aims: We evaluated 17 consecutive patients treated at the Medical University Vienna from 07/04 - 05/09 with the protocol of the GMALL 07/03. Median age was 34 years. Eight patients belonged to the standard risk, 9 patients to the high-risk group. Main focus was set on their adherence to therapy during the different phases of the protocol.

Results: 16/17 patients could not follow the strict time schedule during induction and consolidation therapy. (Table 1) Median delay after consolidation was 30 days (range $3 ; 80$ ) including all patients. Comparing high-risk with standard risk patients after consolidation 1 median delay in high-risk patient 35 years with patients $<35$ years median delay was 46 vs. 17 days. In total only 3 of 8 standard risk patients were able to receive the entire therapy. Reasons were side effects ( 1 pancreatitis, 1 major thrombosis), refractory leukemia $n=1$ and relapse $n=2$.In high risk patients $7 / 9$ patients received stem cell transplantation after consolidation therapy. In total 11/17 patients were transplanted (allogen: $n=9$, autologous $n=2$ )In 14/17 patients dose reductions during induction and consolidation therapy were necessary although supportive care was administered strictly according to protocol.CR could be reached in all patients. $50 \%$ of the patients reached $\mathrm{CR}$ within 45 days. Overall survival was $50 \%$ at 45 months. It is remarkable that we did not observe any difference between standard and high risk patients. the exception and accumulate particularly in standard risk patients after consolidation 1. In high-risk patients delay might not have such an impact because sented.

Disclosure: No conflict of interest disclosed.

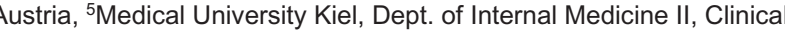
was less than in standard risk patients ( 29 vs. 45 days). Comparing patients > patients are transplanted early. Details and updated follow up will be pre- 
P800

\section{Nodular lesions of the scalp and a mediastinal mass in T-cell acute lymphoblastic leukemia (T-ALL)}

\section{Konik, A. ${ }^{1}$; Dührsen, U. ${ }^{1}$; Röth, $A .{ }^{1}$}

${ }^{1}$ Universitätsklinikum Essen, Klinik für Hämatologie, Essen, Germany

Introduction: Acute lymphoblastic leukemia (ALL) accounts for approximately 20 percent of acute leukemias in adults. The clinical onset of ALL is rarely insidious, and presenting signs and symptoms reflect bone marrow as well as extramedullary involvement by leukemia. Extramedullary involvements include testis, retina, and skin, although virtually any organ can be infiltrated by leukemic blast cells.

Case: A 20-year-old-man presented with a 3-week history of nodular lesions of the scalp which progressed despite topical treatment with glucocorticosteroids. He also had cervical lymphadenopathy and complained of headache, fatigue and blurred vision. His blood count demonstrated pronounced leukocytosis of $114 / \mathrm{nl}$ with $55 \%$ lymphoblasts, moderate thrombocytopenia (83 / $\mathrm{nl})$ and a normal hemoglobin level $(15 \mathrm{~g} / \mathrm{dl})$. The serum levels of lactate dehydrogenase (LDH; $6402 \mathrm{U} / \mathrm{l})$, creatinine $(1.94 \mathrm{mg} / \mathrm{dl})$ and uric acid $(16.1 \mathrm{mg} / \mathrm{dl})$ were significantly elevated, and there were signs of disseminated intravascular coagulation. Microscopic and flow cytometric analyses of blood and bone marrow were consistent with acute lymphoblastic leukemia (ALL) of mature T-cell type. The chest X-ray revealed a mediastinal mass. Treatment was started with oral dexamethason at a dose of $10 \mathrm{mg} / \mathrm{m}^{2}$ per day (d1-5) and cyclophosphamid $200 \mathrm{mg} / \mathrm{m} 2$ per day (d3-5) (German Multicenter ALL Study Group; study protocol GMALL 07/2003). As early as three days after the first dose the skin lesions and the mediastinal mass regressed completely, paralleled by a dramatic improvement of the leukocyte count $(11.83 / \mathrm{nl})$ and the serum values of LDH (1179 U/l) and creatinine $(1.18 \mathrm{mg} / \mathrm{dl})$.

Conclusion: A mediastinal mass is present in over half of patients with newly diagnosed ALL of T-cell origin, but skin lesions are rare and the frequency may be as low as $1 \%$. Skin involvement with acute lymphoblastic leukemia may occur at presentation concomitantly with bone marrow disease, or may be the first sign of relapse, or even may precede bone marrow infiltration. Skin biopsy along with immunohistochemistry may help to establish the diagnosis

Disclosure: No conflict of interest disclosed.

P801

\section{Acute lymphoblastic leukemia and Brugada syndrome}

Schneemilch, M.E. ${ }^{1}$; Malfertheiner, M.2; Tanev, I.2; Fischer, T. ${ }^{1}$; Hütten, $H^{1}$

${ }^{1}$ Otto-von-Guericke Universität, Hämatologie und Onkologie, Magdeburg, Germany, ${ }^{2}$ Otto-von-Guericke Universität, Kardiologie, Angiologie,

Pneumologie, Magdeburg, Germany

Introduction: The Brugada syndrome is an inherited arrhythmia that causes ventricular fibrillation often ending with sudden cardiac death. Studies indicate that Brugada syndrome is responsible for $4 \%-12 \%$ of unexpected sudden deaths. The calculated statistical probability of an independent temporal coincidence between ALL and the Brugada Syndrome is less than 1 per 2 billion. Here, we report a case in which we found a coincidence of ALL and Brugada syndrome. This report provides information about clinical features, diagnosis and treatment strategies considering the combination of both diseases.

Case Report: A 38-year-old Caucasian male was admitted with epigastric pain and unspecific adynamia. His medication at the timepoint of admission was pantoprazol and allopurinol. He had a smoking history of $1 / 2$ pack of cigarettes since 20 years. The physical exam was unremarkable except for adiposity. His blood count on admission was: WBC 17,89 Gpt/l, PLT 39 Gpt/1 and $\mathrm{Hb}$ 6,0 $\mathrm{mmol} / \mathrm{l}$. A diagnosis of a common-ALL with $79 \%$ lymphoblasts in the peripheral blood and subtotal infiltration in the bone marrow was made. On the second day of hospitalization the ECG showed ventricular fibrillation which had to be defibrillated 6 times. Reanimation was started immediately. Intubation was required. He underwent cardiac catheter examination, which showed no relevant stenosis. Typical ECG pattern for Brugada Syndrome type I was found. The Patient was admitted to the intensive care unit. Manifestation of hypoxic brain damage was noted. Induction therapy for cALL was started and applied at the intensive care unit. After hematopoietic recovering an ICD was implanted. The mental status of the patient is currently improving dramatically. Therapy for c-ALL is being continued.

Conclusions: Anemia caused by the cALL probably induced ventricular fibrillation in this patient who was asymptomatic prior to admission. Because of adiposity cardiac massage applied during resuscitation might not have been effective to oxygenate the brain properly. We suggest holding the hemoglobin at a level above 7,0 $\mathrm{mmol} / \mathrm{l}$ in patients with known Brugada syndrome to avoid occurrence of ventricular fibrillation.

Disclosure: No conflict of interest disclosed.

\section{Posterdiskussion}

CLL 2

P802

\section{Novel paradigm to induce apoptosis in chronic lymphocytic leukemia cells}

Patz, M. ${ }^{1,2,3}$; Frenzel, L.P. ${ }^{1,2,3} ;$ Pallasch, C.P.1,2,3; Claasen, J. ${ }^{1,2,3}$; Brinker, R. ${ }^{1,2,3}$; Linde, N.-F. ${ }^{1,2,3}$; Wendtner, C.-M. ${ }^{1,2,3}$

${ }^{1}$ Uniklinik Köln, Klinik I für Innere Medizin, Köln, Germany, ${ }^{2}$ Centrum für Integrierte Onkologie (ClO), Köln, Germany, ${ }^{3}$ Cologne Excellence Cluster on Cellular Stress Responses in Aging-Associated Diseases (CECAD), Köln, Germany

Purpose: Chronic lymphocytic leukemia (CLL) is characterized by an accumulation of mature B-cells that fail to undergo apoptosis. Apoptosis induction is balanced by pro-apoptotic and anti-apoptotic proteins. CLL cells predominantly express higher level of anti-apoptotic molecules. Reasons for deregulated proteins in the apoptosis machinery are rare. In the past years a connection between microRNAs (miRNAs) and protein expression in context of CLL was formed. For some proteins, which are linked to the pathogenesis of CLL, miRNAs play a role in protein expression e.g. PLAG1 and TCL-1. A target of miR-15/16 is the anti-apoptotic protein BCL-2, which is overexpressed in CLL.

Experimental design and results: In a miRNA array comprising $752 \mathrm{miR}$ NAs deregulation of 26 miRNAs was previously reported (Blood 2008;112:4213-9). Based on these findings we transfected primary CLL cells with miRNAs and analyzed apoptosis induction by flow cytometry after 24 and 48 hours. To sensitize CLL cells for apoptosis we further treated them with apoptosis inducing drugs. The protein level of miRNA targeted proteins was assessed in Western Blots. Levels of transfected miRNAs were determined by RT-PCR. After transfection of miRNAs most patients responded with a higher apoptosis induction. Taken all samples together we find a significant higher apoptosis rate compared to control transfected CLL cells.

Conclusion: Here we show for the first time, that aberrantly expressed miRNAs have a strong impact on survival of CLL cells. Further explorations are needed to get better insight to differentially expressed miRNA with respect to the biology of CLL.

Disclosure: No conflict of interest disclosed.

\section{P803}

\section{Regulation of miRNA by stimulating CLL cells}

Patz, M.1,2,3; Frenzel, L.P. ${ }^{1,2,3}$; Pallasch, C.P. ${ }^{1,2,3}$; Wendtner, C.-M. ${ }^{1,2,3}$

${ }^{1}$ Uniklinik Köln, Klinik I für Innere Medizin, Köln, Germany, ${ }^{2}$ Centrum für Integrierte Onkologie (ClO), Köln, Germany, ${ }^{3}$ Cologne Excellence Cluster on Cellular Stress Responses in Aging-Associated Diseases (CECAD), Köln, Germany

Introduction: Chronic lymphocytic leukemia (CLL) B cells fail to undergo apoptosis in vivo. Surprisingly, in vitro these cells are dying very rapidly. Reasons for fast apoptosis induction are attributed to missing stimuli presented by the microenvironment. It is known that stimulation of the B-cell receptor (BCR) leads to a prolonged survival of CLL cells. So far evaluation of BCR stimulation focuses on mRNA expression and involvement of signaling molecules. Our first analysis of miRNA expression was examined on resting cells. In this respect we found 26 deregulated miRNAs in CLL cells compared to peripheral CD19-sorted B-cells. The effects on miRNA expression after 
stimulation of the BCR are unknown. In a new approach we analyzed the effects of BCR stimulation on miRNA expression.

Experimental design and results: For this reason we monitored the miRNA expression profile of 30 CLL samples and 10 samples of CD19 sorted peripheral B-cells with and without BCR stimulation after $0 \mathrm{~h}, 3 \mathrm{~h}$ and $24 \mathrm{~h}$. The miRNA array was performed with an Illumina Bead Chip platform allowing the analysis of 752 miRNAs. The evaluation of the miRNA array shows for all BCR stimulated cells an upregulation of several miRNAs. Seven miRNAs were shown to be upregulated in CLL cells compared to six upregulated miRNAs in healthy B cells. In the following experiments we want to examine the impact of these miRNAs on BCR-stimulation. Comparison with a previously published mRNA array after BCR stimulation will be used to connect both arrays with a bioinformatic approach.

Conclusion: In this approach we examined for the first time the effects of BCR stimulation on miRNA levels on CLL cells and found an upregulation of several miRNAs. Evaluation of pathways that are involved in a prolonged survival of CLL cells and that are linked to miRNAs will contribute to find new therapeutic options for treating CLL.

Disclosure: No conflict of interest disclosed.

\section{P804}

\section{Small molecule XIAP inhibitors sensitize CLL cells for TRAIL-induced apoptosis}

Schnaiter, A.B. ${ }^{1}$; Zenz, ${ }^{1}{ }^{1}$; Löder, S. ${ }^{2}$; Mertens, D. ${ }^{1}$; Winkler, D. ${ }^{1}$; Döhner, H. ${ }^{1}$; Debatin, K.-M. ${ }^{2}$; Silgenbauer, S. ${ }^{1}$; Fulda, S. ${ }^{2}$

${ }^{1}$ Universitätsklinikum UIm, Klinik für Innere Medizin III, UIm, Germany, ${ }^{2}$ Universitätsklinikum UIm, Klinik für Kinder- und Jugendmedizin, UIm, Germany

CLL cells are characterized by an apoptotic defect which leads to accumulation of malignant cells rather than proliferation. High expression levels of inhibitor of apoptosis proteins (cIAP1, cIAP2, XIAP) may explain at least in part their apoptosis resistance. TRAIL, a death receptor ligand which induces apoptosis in various cancer cell lines is not very effective in CLL cells. Thus the aim of this study was to reconstitute the apoptotic machinery by subtoxic concentrations of small molecule XIAP inhibitors and to sensitize CLL cells for TRAIL-induced apoptosis.

CLL cell lines (MEC-1, JVM-2, JVM-3) and ficollized blood samples of 27 well characterized CLL patients were analyzed. Surface expression of TRAIL receptors was detected by FACS, apoptosis by Annexin/PI staining and light scatter analysis, caspase-activation by using a fluorescent caspase-substrate Caspase-cleavage was shown by Western blot analysis.

All CLL cell lines showed high expression of the agonistic TRAIL-R2 receptor. TRAIL induced apoptosis in a dose-dependent manner. This effect was significantly enhanced in combination with non-toxic concentrations of small molecule XIAP inhibitors. Western blot analysis revealed increased cleavage of initiator caspase- 3 , but not caspase- 8 in response to TRAIL plus XIAP inhibitor compared to TRAIL treatment alone. In 18 of $27(67 \%)$ of the primary samples the combination with XIAP inhibitor enhanced TRAIL-induced apoptosis. Caspase- 3 was cleaved into its active fragments in cases which were responsive whereas no cleavage occurred in the unresponsive samples. Highest caspase- 3 activity was reached after 4 to 6 hours and clearly enhanced after combination treatment. XIAP inhibitor plus TRAIL-induced apoptosis was nearly completely blocked by the pan-caspase inhibitor zVADfmk showing caspase-dependency. Importantly, XIAP inhibitor cooperated with TRAIL to trigger apoptosis even in samples derived from patients with unfavorable prognostic factors like $17 \mathrm{p}$-deletion, fludarabine-refractoriness or unmutated VH genes. Strikingly, cells with unmutated VH genes responded significantly better than those with mutated ones.

This study demonstrates for the first time that the combination of small molecule XIAP inhibitors and TRAIL is able to sensitize CLL cell lines as well as primary CLL cells to TRAIL-induced apoptosis, including samples of high risk patients. This combination strategy may open new therapeutic options to reactivate apoptosis programs in CLL.

Disclosure: No conflict of interest disclosed.
P805

\section{Comparison of the in vitro effects of the CD20 antibodies} rituximab and GA101 on CLL cells

\author{
Patz, M. ${ }^{1}$; Forcob, N. ${ }^{1}$; Isaeva, P. ${ }^{1}$; Klein, C. ${ }^{2}$; Umana, P. ${ }^{2}$; \\ Wendtner, C. ${ }^{1}$; Hallek, M. ${ }^{1}$; Krause, G. ${ }^{1}$ \\ ${ }^{1}$ Universität Köln, Innere Medizin I, Köln, Germany, ${ }^{2}$ Roche Glycart AG \\ Schlieren, Switzerland
}

Introduction: In a pre-clinical in vitro assessment using blood samples from chronic lymphocytic leukemia (CLL) patients we compared the effects of two monoclonal antibodies targeting CD20, namely rituximab, the current standard for treatment of Non-Hodgkin lymphoma and CLL in combination with chemotherapy, and GA101, a glyco-engineered type II antibody.

Methods: Antibody-induced phosphatidylserine exposure was examined in isolated CLL cells. In order to additionally monitor Fc-mediated cell killing, B cell depletion assays from whole blood samples were performed.

Results: Treatment with rituximab or GA101 on the average reduced the viability of isolated CLL cells by $6 \%$ or $11 \%$, and the ratio of B to T cells in whole blood samples by $12 \%$ or $33 \%$. Both antibodies enhanced the cytotoxic effects of chemotherapeutic agents on isolated CLL cells, significantly for the combination of chlorambucil and GA101. CD20 surface expression on CLL cells correlated with GA101-induced B cell depletion, which comprises Fc-mediated cell killing, but not with direct cell death induction. Treatment of whole blood samples from CLL patients with a CpG containing oligonucleotide resulted in increased CD20 expression on CLL cells and enhanced GA101-dependent B cell depletion.

Conclusions: As compared to rituximab, GA101 increased phosphatidylserine exposure on isolated CLL cells more strongly and, to an even higher degree, led to superior B cell depletion in whole blood samples, which argues for clinical investigation of GA101 in CLL patients.

Disclosure: Michaela Patz: No conflict of interest disclosed. Günter Krause: Financing of Scientific Research: Roche Glycart AG

\section{P806}

NO donating acetylsalicylic acid derivates for the treatment of chronic lymphocytic leukemia (CLL) in vitro and in vivo

\section{Poll-Wolbeck, S.J. ; Razavi, R. ${ }^{1}$; Gehrke, I. ${ }^{1}$; Hallek, M. ${ }^{1}$; Kreuzer,}

K. - . $^{1}$

${ }^{1}$ Klinik I für Innere Medizin, Universitätsklinikum Köln, Köln, Germany

Introduction: Acetylsalicylic acid (ASA) is known for its antineoplastic properties, but due to major side effects was never used as anti-cancer drug. NO donating ASA (NO-ASA) derivates are substantially better tolerated while the systemic effect is maintained. Chronic lymphocytic leukemia (CLL) remains an incurable disease until today with patients frequently evolving resistance to modern treatment regimes. Therefore, potent substances with low systemic toxicity are highly desirable. The aim of this study was to investigate the effect of different NO-ASA isomers on primary CLL cell survival in vitro and in vivo.

Methods: Primary CLL cells were treated for $24 \mathrm{~h}$ with two NO-ASA isomers NCX4016 and NCX4040 $(1 \mu \mathrm{M}-100 \mu \mathrm{M})$, as well as with ASA and salicylic acid (SA) $(1 \mu \mathrm{M}-20,000 \mu \mathrm{M})$. Apoptosis induction was assessed flow cytometrically by AnnexinV/propidium iodide staining and western blot. Protein expression of relevant survival proteins was also assessed by western blot. The transcriptome was analyzed after NO-ASA treatment of $3 \mathrm{~h}$ by Affymetrix human whole genome arrays. The in vivo efficacy of NC4016 and NCX4040 was tested in a JVM3 xenograft mouse model. Nude mice received $100 \mathrm{mg} / \mathrm{kg}$ bodyweight p.o. of either compound or the corresponding vehicle control substance.

Results: NCX4040 induced apoptosis in CLL cells with an $\mathrm{LC}_{50}$ of $8.72 \mu \mathrm{M}$ $( \pm 0.04 \mu \mathrm{M})$, whereas healthy PMBCs remained largely unaffected. NCX4016 showed a cytotoxic effect only when applied in high concentrations of $>100$ $\mu \mathrm{M}$. The $\mathrm{LC}_{50}$ of ASA and SA was greater than 5,000 $\mu \mathrm{M}$. NCX4040-mediated cytotoxicity was due to apoptosis induction, as activation of the caspases 3, 9 and PARP cleavage occured. Furthermore, NCX4040 significantly reduced tumor growth (IR 83.4\%) in the JVM3 xenograft mouse model, while NC4016 
was completely ineffective. Transcriptome analysis of treated versus untreated primary CLL cells revealed differential expression of 213 genes in NCX4040 treated samples. Most genes were upregulated, including transcription factors like $c$-jun, fos and sod1.

Discussion: The high cytotoxic effect of NCX4040 was not due to its acetylsalicylic acid domain, since the $\mathrm{LC}_{50}$ for ASS and SS were at least 700 fold higher. Nevertheless, the positioning of the NO-domain seemed to be important as only one isomere (NCX4040) showed potent antineoplastic efficiency. NCX4040 can therefore be considered a promising compound for CLL treatment. Further analysis of differentially expressed genes upon treatment might reveal more about the potential.

Disclosure: No conflict of interest disclosed.

\section{P807}

\section{Possible role of lymphoid enhancer binding factor-1 (LEF-1) for cell survival and disease progression in chronic lymphocytic leukemia (CLL)}

\section{Poll-Wolbeck, S.J. ${ }^{1}$; Erdfelder, F. ${ }^{1}$; Gehrke, I. ${ }^{1}$; Hallek, M. ${ }^{1}$; Kreuzer, K.-A. ${ }^{1}$ \\ ${ }^{1}$ Universität zu Köln, Klinik I für Innere Medizin, Köln, Germany}

Introduction: Several array based transcriptome analyses showed overexpression of the transcription factor LEF-1 in CLL cells whereas this gene is virtually silenced in healthy B cells. The aim of our study was to elucidate the functional role of LEF-1 in the development and the progression of this disease.

Methods: We used the selective small molecules CGP049090 and PKF115584 to antagonize LEF-1 function in vivo (nude mice xenografted with JVM3 cells) and in vitro (western blot, AnnexinV/propidium iodide assay), as well as quantitative RT-PCR methods to correlate LEF-1 expression of CLL samples with known risk factors and disease progression.

Results: The relative expression ratio (RER) of LEF-1 mRNA was highly correlated with the ZAP70 status $\left(\mathrm{ZAP70}^{+}=53.7\right.$ vs. $\mathrm{ZAP70}^{-}=37.1$; Mann Whitney $U=866.50, p=0.004)$. Additionally, patients with high LEF-1 expression exhibited a higher percentage of malignant cells in the peripheral blood (by median split LEF-1 $1^{\text {high }}=88.4 \%$ and $L E F-1{ }^{\text {low }}=76.3 \%$; Mann-Whitney $\mathrm{U}=828.50, \mathrm{p}<0.001)$. Further, comparison of LEF-1 expression in newly diagnosed Binet A with those in patients with Binet A/B that require treatment and Binet $C$ revealed a RER of 22.0 in newly diagnosed patients and 85.6 for patients that require treatment. This difference was highly significant (MannWhitney $\mathrm{U}=341.50, \mathrm{p}<0.001)$. Our in vitro and in vivo experiments with the LEF-1 antagonists show that this transcription factor is essentially involved in CLL cell survival. CGP049090 and PKF115-584 induced caspase-dependent apoptosis in vitro at low $\mu \mathrm{M}$ concentrations $\left(\mathrm{LC}_{50} 0.7 \mu \mathrm{M}\right.$ and $\left.0.9 \mu \mathrm{M}\right)$ in malignant cells, whereas healthy B cells, which express LEF-1 only at very low levels, were largely unaffected. Both substances also significantly reduced tumor volume growth $(>70 \%)$ in a LEF-1-positive JVM3-xenograft mouse model.

Conclusion: Strong positive correlations of LEF-1 with adverse prognostic markers such as ZAP70-positivity and high lymphocyte counts suggest a role of LEF-1 in the progression of CLL. Therefore, LEF-1 might also be useful as a marker for disease severity and the need of treatment. In addition, as the activity of LEF-1 appears to be critically involved in the survival of CLL cells, this transcription factor and also its activating upstream signalling cascade, the WNT-pathway, may be potent targets for drug development in CLL.

Disclosure: No conflict of interest disclosed.

\section{P808}

\section{Selective anti-leukemia activity of catalytic redox 'sensor/ effector' agents}

\section{Lilienthal, N. ${ }^{1}$; Peer-Zada, A.A. ${ }^{1}$; Ba, L.A. ${ }^{2}$; Liu, H. ${ }^{1}$; Jacob, C. ${ }^{2}$;} Hallek, M. ${ }^{1}$; Herling, M. ${ }^{1}$

${ }^{1}$ Uniklinik Köln, Klinik I für Innere Medizin, Köln, Germany, ${ }^{2}$ Universität des Saarlandes, Saarbrücken, Germany

Introduction: Reactive oxygen species (ROS) are highly active molecules and normal by-products during oxygen metabolism in cellular respiration. Dysfunctions of these mitochondrial processes result in enhanced accumulation of ROS which promotes genotoxic stress. This in turn propels cellular aging and mutagenesis. Consequently, cells characterized by oncogenically dictated growth behavior show elevated ROS levels. Here, we test if this high redox burden of tumor cells (chronic lymphocytic leukemia; CLL) in contrast to their normal counterparts confers a specific vulnerability towards selective targeting. Methods: We designed a panel of novel redox-active "sensor/effector" compounds (i.e. organotellurides) that should by chemical inference be capable of elevating ROS levels preferentially in those cells that already carry an abnormally high redox burden. Their in vitro activity as compared to negative and positive (pro-survival stimulus) controls was investigated in peripheral blood B-cells from CLL patients and those of healthy donors. Analysis included molecular markers of cell death induction (i.e. AnnexinV-PE/7AAD staining, Caspase activation, or apoptotic protein expression).

Results:

1) ROS levels in CLL cells are substantially higher as compared to normal B-cells of the same patient or to those from healthy individuals.

2) Treatment with our novel redox catalysts in vitro results in further elevation of ROS levels to a much higher degree in CLL cells than in normal leukocytes.

3) ROS increases in CLL cells are followed by massive specific apoptosis.

4) There is a marked synergism of in vitro compound activity with conventional DNA-targeting chemotherapeutics (i.e. fludarabine).

5) Treatment-induced substantial increases of glutathione levels in normal blood lymphocytes stand against largely unaffected levels of this "redoxbuffer" in CLL cells.

Conclusions: We demonstrate a highly selective anti-leukemia activity of novel synthesized catalytic redox 'sensor/effector' agents. This is triggered by an increase of abnormally elevated ROS levels and subsequent induction of the otherwise insufficient apoptotic machinery. Additionally, CLL cells show inherently reduced activity of redox compensations (glutathione levels). Our findings support the proposed link between mitochondrial redox-mediated signaling and cell death. They encourage further pre-clinical investigations and synthetic optimizations towards potential anti-leukemic treatment options.

Disclosure: No conflict of interest disclosed.

\section{P809}

\section{Cryptochrome 1 (CRY1) promoter methylation is associated with good prognosis in B-cell chronic lymphocytic leukemia (CLL)}

\section{Hanoun, M. ${ }^{1}$; Klein-Hitpass, L. ${ }^{2}$; Eisele, L. ${ }^{1}$; Dührsen, U. ${ }^{1}$; Dürig, J. ${ }^{1}$}

${ }^{1}$ University Hospital Essen, West German Cancer Center , Department of Hematology, Essen, Germany, ${ }^{2}$ Institute of Cell Biology, University of Essen-Duisburg, Essen, Germany

Introduction: Disruption of circadian rhythm is believed to play a critical role in cancer development. We have previously shown that the expression of the circadian gene CRY1 is an independent prognostic marker in chronic lymphocytic leukemia (CLL). Furthermore, recent data suggest an important functional role of CRY1 in lymphomagenesis where loss of CRY1 reduces the cancer risk in p53 mutant mice. However, the molecular mechanisms underlying deregulated CRY1 expression in CLL remain obscure.

Materials and Methods: To investigate whether epigenetic mechanisms contribute to deregulated CRY1 expression in CLL, we studied DNA methylation within the promoter region of the CRY 1 gene. Methylation studies were performed employing sequencing of cloned PCR products generated from bisulfite-modified DNA extracted from immunomagnetically purified CD19+ 
B cells from the peripheral blood of CLL $(\mathrm{N}=16)$ and normal donors $(\mathrm{N}=5)$. Methylation patterns were correlated with CRY1 mRNA levels as determined by real-time quantitative PCR. Furthermore, mRNA levels of the circadian feedback loop genes comprising CLOCK, BMAL1, CRY1 and PER1-3 were measured.

Results: We confirmed the strong association between CRY1 expression and CD38/ZAP70 expression as well as IgVH mutational status that was previously reported by our group. Of note, CRY1 expression levels in high risk CLL patients (CD38+ZAP70+ and unmutated IgVH status) were comparable to those of normal control donors and significantly higher than in low risk CLL patients suggesting that abnormal underexpression of CRY1 is associated with an indolent clinical course of the disease. We found almost complete demethylation in B cells from patients with high CRY1 expression and normal control donors while patients with low CRY1 expression were characterized by a varying degree of CRY1 promoter methylation $(p<0.0001)$. An intact circadian feedback loop is reflected by high correlation between CRY1 and CLOCK resp. BMAL1 expression which could be shown to be lost in low risk CLL patients $(\mathrm{N}=26)$.

Conclusion: CRY1 is a novel prognostic marker in CLL and is associated with other molecular risk factors. The significant inverse correlation between the degree of methylation and CRY1 expression suggests a causal role of DNA methylation in the deregulation of CRY1 expression. Aberrant methylation of CRY1 may cause disruption of the circadian feedback loop and may explain in part the less aggressive behaviour of CD38-ZAP70- CLL cases.

Disclosure: No conflict of interest disclosed.

\section{P810}

\section{SYK carries no activating point mutations in patients with chronic lymphocytic leukaemia (CLL)}

Mertens, D. ${ }^{1,2}$; Philippen, A. ${ }^{1}$; Diener, S. ${ }^{1}$; Döhner, H. ${ }^{1}$; Stilgenbauer, S. $^{1}$

${ }^{1}$ Uniklinik UIm, Innere III, UIm, Germany, ${ }^{2} \mathrm{DKFZ}$, Mechanisms of Leukemogenesis, Heidelberg, Germany

Introduction: Spleen tyrosine kinase (SYK) is a key mediator of proxima B-cell receptor (BCR) signaling that contributes to resistance to apoptosis chronic lymphocytic leukemia (CLL). In this study, we investigated whether enhanced activity of SYK that has been described in CLL is caused by activating mutations.

Methods: To this end, the complete open reading frame (ORF) of the SYK gene was sequenced in a representative cohort of CLL patients and healthy donors $(\mathrm{N}=46)$.

Results: The analysis of all protein coding exons did not reveal any poin mutation or abnormal SNP allele distribution in patient samples. Taking exonintron-boundaries into account, a new nucleotide polymorphism in close vicinity to the $5^{\prime}$ splice acceptor site of exon 10 was detected in both, patien and healthy samples, with a frequency of $10 \%$ of an enlarged patient cohort ( $\mathrm{N}$ =136). The discovered nucleotide variant did not have any impact on correct splicing of the SYK gene.

Conclusions: Our data demonstrate overall sequence homogeneity of SYK in patients with CLL, excluding the presence of genetic mutations with a prevalence of more than $2 \%$. As SYK is a very interesting therapeutic target in CLL with several clinical trials evaluating inhibitors in CLL, the underlying molecular cause for enhanced SYK activity remains an important question to be adressed.

Disclosure: No conflict of interest disclosed.
P811

Sustained NF-kappa B activity is A20-independent in CLL

Frenzel, L.P.1,2,3; Claus, R. ${ }^{4}$; Plume, N. ${ }^{5}$; Schwamb, J.1,2,3; Pallasch, C.P. ${ }^{1,2,3}$; Claasen, J. ${ }^{1,2,3}$; Brinker, R. ${ }^{1,2,3}$; Wollnik, B. ${ }^{5}$; Plass, C. ${ }^{4}$ Wendtner, C.-M.1,2,3

${ }^{1}$ Uniklinik Köln, Klinik I für Innere Medizin, Köln, Germany, ${ }^{2}$ Centrum für Integrierte Onkologie (CIO), Köln, Germany, ${ }^{3}$ Cologne Excellence Cluster on Cellular Stress Responses in Aging-Associated Diseases (CECAD), Köln, Germany, ${ }^{4}$ Deutsches Krebsforschungszentrum, Abteilung für Epigenomik und Krebsrisikofaktoren, Heidelberg, Germany, ${ }^{5}$ Institute of Human Genetics and Center for Molecular Medicine Cologne (CMMC), Köln, Germany

Purpose: Inappropriate Nuclear Factor kappa B (NFkB) activity is one major hallmark of B cell-malignancies as well as chronic lymphocytic leukemia (CLL). NFкB-dependent genes are involved in anti-apoptosis (e.g. IAP-1, IAP-2, XIAP and TOSO), cell proliferation and metastasis and are responsible for survival and proliferation of tumors. However, the mechanisms of NFKB overexpression in CLL still need to be elucidated. Previously we identified translocations in a region on chromosome $6 \mathrm{q}$ that encodes tumor necrosis factor alpha-induced protein 3 (TNFAIP3, A20), which is a key player in negative feedback loop regulation of NFKB. Inactivation of this ubiquitin-editing enzyme is involved in immunopathologies (e.g. Crohn's disease, rheumatoid arthritis, systemic lupus erythematosus, psoriasis and type 1 diabetes) and in tumorigenesis. Frequent mutations in the A20 locus - leading to sustained $\mathrm{NF} \mathrm{B}$ activity - could be shown to play a dominant role in development of different B-cell malignancies (e.g. Hodgkin disease, DLBCL and MZL), but were absent in FL and MCL. These observations show that these malignant B cell-entities share similarities concerning major events in their tumorigenesis. Experimental design and results: In order to check if A20 is involved in upregulation of NF-kappa B activity in CLL, we sequenced exons 2-9 of the tnfaip3 gene in 70 CLL DNA samples. Furthermore, we determined the methylation status of the promoter region and compared to 10 control DNAs of B-cells from healthy donors. Contrary to reports from other B cell-malignancies the A20 region showed neither mutations nor methylations. Moreover its expression could be confirmed by Immunoblotting and showing comparable results to healthy $\mathrm{B}$ cells.

Conclusion: These results indicate that malignant development in CLL differs from those of HD, DLBCL and MZL but share similarities with FL and MCL, since A20 is still a functional tumor-suppressor in FL, MCL and CLL.

Disclosure: No conflict of interest disclosed.

P812

The lipid metabolism as a new target in chronic lymphocytic leukemia (CLL)

\section{Frenzel, L.P.1,2,3; Feldhaus, V.1,2,3; Pallasch, C.P.1,2,3.}

Wendtner, C.-M. ${ }^{1,2,3}$

${ }^{1}$ Uniklinik Köln, Klinik I für Innere Medizin, Köln, Germany, ${ }^{2}$ Centrum für Integrierte Onkologie (CIO), Köln, Germany, ${ }^{3}$ Cologne Excellence Cluster on Cellular Stress Responses in Aging-Associated Diseases (CECAD), Köln, Germany

Purpose: The accumulation of the malignant chronic lymphocytic leukemia (CLL) cells seems to be caused not only by failed apoptosis but also by changes in cell cycle regulation. Analysis of gene expression profiles revealed significant changes in lipid metabolism associated genes when comparing CLL cells with healthy controls (Leukemia, 2008. 22(3):585-92). The majority of genes in this cluster exhibited phospholipase activity, but proteins like lipoprotein lipase (LPL) were also identified as over-expressed in CLL. One aim of this project is to investigate the influence of enhanced lipase activity on membrane stability and composition with respect to formation and composition of lipid rafts.

Methods: Changes in lipid composition (e.g. cholesterol or phospholipids) were analyzed by thin layer chromatography. CLL cells and healthy B cells were freshly isolated and subsequently processed to reveal liquid extracts which were applied to silica gel, separated by lipophilic mobile phase and visualized by lipid staining. 
Results: We demonstrate that several classes of phospholipids, namely phosphatidyl-ethanolamine, -inositol, -serine, -choline, -glycerol and cholesterol are deregulated in CLL cells compared to healthy B cells.

Conclusion: We assume that deregulation of phospholipids and cholesterol leads to changes in membrane composition of CLL cells. This may result in different cell signaling, for example in terms of survival and proliferation and mitochondrial function. Lipid metabolism indeed seems to be a new evolving field in CLL research, which has been so far only analyzed for its prognostic relevance, but remains to be functionally elucidated to reveal new therapeutic strategies.

The work is supported by a grant from the German Cancer Aid/ Deutsche Krebshilfe (DKH109159).

Disclosure: No conflict of interest disclosed.

\section{P813}

\section{Where do CLL cells originate from? A micro-RNA approach...}

Patz, M. ${ }^{1,2,3}$; Frenzel, L.P. ${ }^{1,2,3} ;$ Pallasch, C.P. ${ }^{1,2,3}$; Wendtner, C.-M. ${ }^{1,2,3}$

${ }^{1}$ Uniklinik Köln, Klinik I für Innere Medizin, Köln, Germany, ${ }^{2}$ Centrum für Integrierte Onkologie (CIO), Köln, Germany, ${ }^{3}$ Cologne Excellence Cluster on Cellular Stress Responses in Aging-Associated Diseases (CECAD), Köln, Germany

Introduction: The origin of CLL cells is discussed controversially. Three candidates are discussed to be the origin of CLL cells, namely: $\mathrm{CD}^{+} \mathrm{B} 1$ cells, memory B-cells or all other CD19+ B-cells subpopulations. Examination of the expression of surface molecules on CLL cells appear to be similar to memory B cells. However, CLL cells carrying unmutated $\mathrm{IgVH}$ could arise from marginal B cells. Another hypothesis suggests $\mathrm{CD}^{+} \mathrm{B} 1$ cells as CLL progenitors. These cells express CD5, the marker for CLL B cells, that is usually expressed on $\mathrm{T}$ cells.

Experimental design and results: To examine miRNA expression between CLL cells $(n=29)$ and their potential subsets of origin, B cells were collected from buffy coats. Memory B-cells $(n=8)$ and B-1 cells $(n=8)$ were collected via MACS-sorting using CD19/CD27 or CD19/CD5 coated magnetic beads, peripheral B cells were collected using CD19 coated $(n=10)$ magnetic beads. The miRNA expression was monitored by using Illumina Bead Chip platform comprising 752 miRNAs. Comparison between these three B cell subsets and CLL cells revealed 38 differentially regulated miRNAs compared to peripheral CD19-positive B cells, 35 differentially regulated miRNAs compared to B-1 cells and 43 ones compared to memory B cells $(p<0,001)$. Interestingly many of the differentially regulated miRNAs found in each subset were overlapping.

Conclusion: Compared to different B cell subsets, CLL cells show a distinct modified expression of miRNA, so that no clear conclusion regarding their origin can be drawn. Further analysis grouping CLL cells in their prognostic subsets will be done and compared to the intersecting and differential miRNAs of all groups.

Disclosure: No conflict of interest disclosed.

P814

\section{Leptomeningeal involvement of chronic lymphocytic leukemia: A report of two patients successfully treated with combined chemotherapy and liposomal cytarabine (DepoCyte)}

Pohlen, M. ${ }^{1}$; Kerkhoff, A. ${ }^{1}$; Kessler, T.1 ; Müller-Tidow, C. ${ }^{1}$; Berdel, W.E. ${ }^{1}$; Koschmieder, S. ${ }^{1}$

${ }^{1}$ Universitätsklinikum Münster, Medizinische Klinik und Poliklinik A, Münster, Germany

Introduction: Leptomeningeal involvement in patients with chronic lymphocytic leukemia (CLL) is rare, and optimal management of these patients is presently unclear.
Methods: We describe the successful treatment of two CLL patients with documented leptomeningeal involvement using chemotherapy as well as liposomal cytarabine (LC).

Results: Two patients with neurological symptoms were diagnosed with B-CLL. The first patient (46 yrs; Binet B) was admitted with hemiplegia. MRI showed cerebral hyperintensity while examination of spinal fluid (249 cells/ $\mu 1)$ confirmed infiltration by CD20+ lymphoid cells. The patient received 6 cycles of R-FC. LC instead of conventional intrathecal triple therapy (cytarabine, methotrexate, prednisone) was administered to achieve a long-lasting cytotoxic effect in spinal fluid. Already after first application, neurological symptoms rapidly and completely disappeared, and cell numbers $(6 / \mu \mathrm{l})$ and morphology normalized (Figure 1).

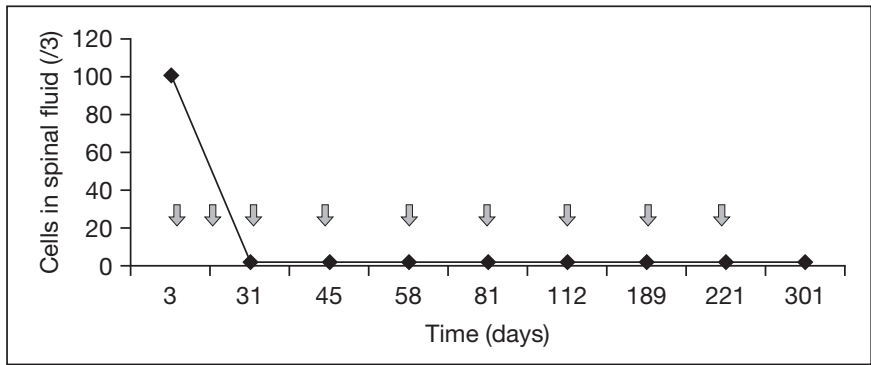

Fig. 1.

This patient has received LC in 2-weekly intervals for 32 weeks, after which treatment was stopped because of ongoing CR (>12 months). In addition to this patient, feasibility of administration of LC in CLL with CNS involvement was confirmed in a second patient with B-CLL (49 yrs, Binet C) with headaches and diplopia caused by leptomeningeal involvement. This patient had already received 7 conventional triple therapies paralleled by 6 cycles of R-FC, when application of LC was started in order to decrease frequency of further intrathecal therapies. Follow-up visits ( $>20$ months) confirmed a complete neurologic and hematologic remission. In both patients, no significant adverse events related to LC administration were observed.

Conclusions: Conventional intrathecal treatment is limited by the short cerebrospinal half-life of cytarabine, necessitating frequent administration (at least twice a week). Here, we demonstrate that application of LC, together with and following R-FC, in patients with B-CLL and CNS involvement is safe and effective. This regimen allows to decrease the frequency of intrathecal therapy to a once-in-two-weeks administration with a subjective increase of quality of life of this rare patient group.

Disclosure: No conflict of interest disclosed.

\section{P815}

\section{Rare case of hemolytic anemia}

Kühr, T.1; Wallner, G. ${ }^{2}$; Webersinke, G. ${ }^{3}$; Marschon, R. ${ }^{3}$; Thaler, J. ${ }^{1}$; Petzer, A.L. ${ }^{4}$; Tschurtschenthaler, G. ${ }^{4,5}$

${ }^{1}$ Klinikum Wels-Grieskirchen, Abteilung für Innere Medizin IV, Wels, Austria, ${ }^{2}$ Klinikum Wels-Grieskirchen, Institut für med. und chem. Labordiagnostik II, Wels, Austria, ${ }^{3}$ Krankenhaus Barmherzige Schwestern Linz, Labor für Molekularbiologie und Tumorzytogenetik, Linz, Austria, ${ }^{4}$ Krankenhaus Barmherzige Schwestern Linz, Abteilung für Innere Medizin I, Linz, Austria, ${ }^{5}$ Abteilung für Innere Medizin I, Labor für hämatologische Spezialdiagnostik, Linz, Austria

Introduction: Pathologic cold agglutinins are produced either in response to infection or by paraneoplastic or neoplastic growth of a single immunocyte clone. We report a case of interest, where cold agglutinin disease revealed a T-cell large granular lymphocyte (LGL) leukemia.

Methods: Diagnostic workup included radiological, microbiological, laboratory, and profound immunophenotypic profiling of the peripheral blood and bone marrow.

Results: A 47-year-old man presented with anemia ( $\mathrm{Hb}: 7.2 \mathrm{~g} / \mathrm{dl}$ ) neutropenia $(0,6 \mathrm{G} / \mathrm{L})$, an elevated serum lactate dehydrogenase of $443 \mathrm{IU} / 1$ with no detectable Haptoglobin. A direct Coombs' test was positive, showing anti-C3d loading. The cold agglutinine titer was 1:64 with an anti-I specificity. Both viral and bacterial serologies were negative. CT-scans and ultrasound did not reveal 
any pathological features. Treatment with steroids and subsequently with Rituximab was commenced. Both systemic interventions, however, were of limited success. Repeated immunophenotype analysis revealed $\mathrm{T}$ cells with a CD3+, CD57+, CD8+, CD7+, CD2+, TCR gamma/delta+, CD56- phenotype. In addition, clonality of the T cell population was proven by PCR. In view of the limited experience to treat T-LGL leukemia, methotrexate was started at an oral dose of $4 \mathrm{mg}$ and gradually increased to $16 \mathrm{mg} /$ week. At 7 -weeks followup, the neutrophils recovered, the direct Coombs' test became negative and the cold agglutinin titer dropped to 1:16. Ever since, the patient did not require any red blood cell transfusions while hemoglobin continuous to rise.

Conclusion: In hemolytic anemia an extended flow cytometric T-cell panel is recommended to detect T-LGL leukemia and offer causal therapy.

Disclosure: No conflict of interest disclosed.

\section{P816}

\section{Drug utilisation and outcomes research on immunoglobulins in a non-interventional long-term outcomes study: Rationale and aims of SIGNS}

\section{Hensel, M. ${ }^{1}$; Borte, M. ${ }^{2}$; Kirch, W. ${ }^{3}$; Faßhauer, M. ${ }^{2}$; Pittrow, D. ${ }^{3}$; Gold, R. ${ }^{4}$, SIGNS Studiengruppe}

${ }^{1}$ Mannheimer Onkologiepraxis, Mannheim, Germany, ${ }^{2}$ Klinikum St. Georg, Fachbereich Pädiatrische Rheumatologie, Infektiologie und Immunologie, Leipzig, Germany, ${ }^{3}$ Institut für Klinische Pharmakologie der TU Dresden, Dresden, Germany, ${ }^{4}$ Klinik für Neurologie, St. Josef-Hospital, Bochum, Germany

Introduction: Patients with primary (mostly genetic) immunodeficiencies, or secondary (acquired) immunodeficiencies for example due to lymphoproliferative disorders (myeloma, chronic lymphocytic leukaemia etc.) have an increased risk of recurrent, life-threatening infections. As standard of care for replacement therapy, such patients receive regularly human immunoglobulin (IgG) preparations intravenously or subcutaneously. IgG infusions are also used for immunomodulation in neurological autoimmune diseases such as Guillain-Barré syndrome.

Methods: The non-interventional prospective open-label cohort SIGNS study ("Assessment of Immunoglobulins in a long-term non-interventional study") was approved by the ethics committee of the TU Dresden and has been initiated to document the utilisation of $\operatorname{IgG}$ in a broad range of indications in clinically relevant situations, not all of which have been assessed by randomised controlled trials. Further, the study aims to provide data on the outcomes of $\mathrm{IgG}$ therapy in everyday clinical practice. Led by an interdisciplinary steering board, hospital- and office-based investigators in 30-40 centres throughout Germany (oncologists, neurologists, paediatricians, other) will document approximately 300 patients and will follow them up for at least two years. Patients of both genders and any age are eligible if they have previously received or are newly scheduled for IgG therapy, for primary, severe secondary immunodeficiency or neurological autoimmune diseases, and have provided written informed consent. No exclusion criteria have been defined in order to minimize selection bias. Long-term outcome data will be collected on patien characteristics in the various indications, drug utilisation (e.g. treatment and dosing patterns), effectiveness (i.e., number of infections), tolerability, health related quality of life, and economic variables (number of hospitalisations, sick-leave days etc. with the option to estimate direct costs). Data collection in SIGNS is performed using a secure (SSL) internet site. Several quality measures are routinely performed including automated plausibility checks at data entry, queries, and on-site monitoring with source data verification.

Conclusion: It is expected that the SIGNS study will contribute to optimization of therapy in this diverse patient population including individuals with various forms of lymphoproliferative disorders.

Disclosure: Manfred Hensel: Advisory Role: Baxter

Ralf Gold: Advisory Role: Baxter

\section{P817}

\section{Chylothorax in a patient with chronic lymphocytic leukemia}

\section{Scholz, G.A. ${ }^{1}$; Sirbu, H. ${ }^{2}$; Semrau, S. ${ }^{3}$; Schauenberg, P. 1 ;} Mackensen, A. ${ }^{1}$; Spriewald, B.M. ${ }^{1}$

${ }^{1}$ Universitätsklinikum Erlangen, Medizinische Klinik 5 - Hämatologie und Internistische Onkologie, Erlangen, Germany, ${ }^{2}$ Universitätsklinikum Erlangen, Thoraxchirurgische Abteilung, Erlangen, Germany, ${ }^{3}$ Universitätsklinikum Erlangen, Strahlenklinik, Erlangen, Germany

Introduction: Chylothorax is defined by the appearance of chyle in the pleural space as a result of a disruption in the thoracic duct. It is commonly associated with nontraumatic etiologies and the most underlying cause is malignancy like lymphoma. However, in association with chronic lymphocytic leukemia (CLL) the occurrence of chylothorax is rare and the best therapeutic option not yet established.

Case presentation: A 65-year-old male patient with first diagnosis of CLL (Binet stage B) presented with hepatosplenomegaly, enlarged cervical, axillary and mediastinal lymph nodes and a pleural effusion on the right side. Analysis of the milky pleural fluid revealed triglyceride levels $>700 \mathrm{mg} / \mathrm{dl}$ and cholesterol levels $<70 \mathrm{mg} / \mathrm{dl}$, establishing the diagnosis of chylothorax. Therefore the patient received 4 courses of immuno-chemotherapy consisting of fludarabine, cyclophosphamide and rituximab (FC-R), resulting in a good clinical response of the CLL. In addition to initiation of the immuno-chemotherapy the patient received total parenteral nutrition over 2 weeks, which resulted in strong reduction of the pleural effusion. However, after restarting enteral lowfat diet the chylothorax relapsed and weekly pleural drainage of $1.500-2.500$ millilitres became necessary. Because of the persisting chylothorax despite immuno-chemotherapy the patient received a percutaneous radiotherapy of the thoracic duct and mediastinum with an overall dose of 24 Gy. However, 8 weeks after radiotherapy the chylothorax still persisted and the patient agreed to a surgical intervention. A decortication of the right lung because of an intraoperatively diagnosed pleural fibrosis and a ligation of the thoracic duct via a muscle sparing thoracotomy were carried out. Following ligation the pleural effusions ceased completely.

Conclusion: Chylothorax caused by CLL may not respond to immuno-chemotherapy nor radiation therapy. Outpatient management using repetitive pleural drainage can be safe as bridging until definite surgical treatment.

Disclosure: No conflict of interest disclosed.

\section{P818}

Combined chemotherapy of Rituximab, Fludarabine, and Cyclophosphamide (R-FC) is superior in younger patients with B-CLL with respect to molecular remission rates

\section{Gebhard, S. ${ }^{1}$; Distelrath, A. ${ }^{1}$; Grothe, W. ${ }^{1}$; Höffkes, H.-G. ${ }^{1}$ ${ }^{1}$ Klinikum Fulda, Tumorklinik, Fulda, Germany}

Purpose: Fludarabine monotherapy is the standard first-line chemotherapy for patients with B-cell chronic lymphocytic leukemia (BCLL). However, there is evidence that the combined chemotherapy of rituximab, fludarabine, and cyclophosphamide (R-FC) is superior in younger patients with B-CLL with respect to molecular remission rates. We present a case series treated with this regimen.

Patients and methods: Previously untreated and symptomatic patients received 5 to 6 cycles of combined chemotherapy of rituximab $\left(375 \mathrm{mg} / \mathrm{m}^{2}\right.$, loading dose $\left.500 \mathrm{mg} / \mathrm{m}^{2}\right)$ day (d) 1 , fludarabine $\left(30 \mathrm{mg} / \mathrm{m}^{2}\right)$ and cyclophosphamide $\left(250 \mathrm{mg} / \mathrm{m}^{2}\right)$ from d 1 to $\mathrm{d} 4$, IV every 4 weeks. Inclusion criteria were: diagnosis of B-CLL according to the Rai classification at stage I to IV, $<75$ years of age, ECOG status 0-1. Primary objectives were Grade 3 or 4 hematologic or biochemical toxicity according to the WHO classification, major bleedings, and infections. Secondary objectiveswere the rate of complete remission and the overall rate of response (complete + partial remission).

Results: Eight patients, 4 male and 4 female, aged mean 59,3 \pm 7,9 years (range: 49 to 73 years) were considered eligible. There was a median of $5.9 \pm$ 0.3 treatment cycles. R-FC was well tolerated with a few episodes of Grade 3 or 4 hematologic toxicities including bleeds that required in two patients twice or more transfusions of packed red cells but no severe infections or biochemical (creatinine, AST, bilirubin) toxicity, there was treatment with G-CSF given 
in 4 patients $(50 \%)$. Two patients had a lack of FC in one cycle due to neutropenia toxicity and one patient had to stop chemotherapy after the 5th cycle due to time delay and toxicity. Seven patients $(88 \%)$ achieved an overall rate of response (4 patients $(50 \%)$ achieved a complete remission, 2 patients $(25 \%)$ achieved a partial remission), and one patient suffered progressive disease after 7 months of partial remission. This patient showed as expected a deletion of TP53 and subsequent low levels of miR-34a. The median duration of followup was $19 \pm 12,1$ months (range 9 to 44 months, 1 patient was lost to follow up after 9 and one patient after 11 months).

Conclusion: Conventional chemotherapy of fludarabine monotherapy on five consecutive days every 4 weeks has been proposed the standard of treatment in patients with B-CLL. This present trial underlines, that R-FC is well tolerated despite Grade 3 or 4 toxicity. These results need to be confirmed in a larger trial.

Disclosure: No conflict of interest disclosed.

\section{Posterdiskussion Multiples Myelom 2 \\ P819 \\ Use of lenalidomide (len) may overcome the adverse prognosis of reduced renal function (RF) in patients (pts) with relapsed/refractory multiple myeloma (MM)}

Kleber, M. ${ }^{1}$; Ihorst, G. ${ }^{2}$; Kohlweyer, U. ${ }^{1}$; Koch, B. ${ }^{3}$; Engelhardt, M. ${ }^{1}$ ${ }^{1}$ University of Freiburg, Hematology and Oncology, Freiburg, Germany, ${ }^{2}$ University of Freiburg, Institute of Medical Biometry and Medical Informatics/Center of Clinical Trials (ZKS), Freiburg, Germany, ${ }^{3}$ University of Freiburg, Central Laboratory, Medical Center, Freiburg, Germany

Introduction: MM-induced renal impairment (RI) is a dreaded complication in MM, formerly being associated with diminished progression-free- (PFS) and overall-survival (OS). Len is an effective novel substance, recently suggested to overcome the prognostic impact of RI to a similar extend as other agents. Since RI - when carefully monitored (Kleber, EJC 2009) - is common in MM, we evaluated PFS and OS under len-treatment in pts with deteriorating RF

Methods: Forty-five consecutive MM pts received len in G1-4 treatment groups ( $\mathrm{G} 1=$ len 25mg/dex 40mg [n=19]; G2: len 25mg/low-dose dex-[n=10]; G3: len 10mg [n=11]; G4: len $10-15 \mathrm{mg}$ plus chemotherapy [n=5]). Serum creatinine (crea) and eGFR were thoroughly monitored and MM-response according to EBMT-criteria.

Results: The median pt age was 66 years (range; 44-78), with pronounced stage II/III disease (Durie\&Salmon 97\%; ISS: 71\%). Pretreatment was considerable with $\geq 2$ previous therapy lines in $71 \%$ and autologous or allogeneic stem cell transplantations performed in $49 \%$ and $11 \%$, respectively before leninitiation. The median len-duration was 20 weeks (range; 4-136), inducing an ORR $(\mathrm{CR} / \mathrm{PR})$ in $27 \%(\mathrm{n}=12)$, clinical benefit rate $(\mathrm{CBR}=\mathrm{CR}+\mathrm{PR}+\mathrm{MR})$ in $37 \%(n=14)$ and disease stabilisation rate $(C B R+S D)$ in $95 \%(n=43)$. Len was well tolerated with WHO-CTC-grade $(\mathrm{G}) \geq 2$ in 10 pts $(22 \%)$ : 4 with neutropenia $(\mathrm{G} 2+3)$. Although baseline RF appeared normal with a median crea of $1.0 \mathrm{mg} / \mathrm{dl}$ (range; 0.6-2.7), nevertheless, mild RI was readily detectable via eGFR (median $83 \mathrm{ml} / \mathrm{min} / 1.73 \mathrm{~m}^{2}$, range; $27-187$ ). Of note, mild $(\mathrm{eGFR}<90$ ) and moderate RI $(\mathrm{eGFR}<60)$ before len-initiation was prominent in $62 \%$ and $31 \%$, respectively, demonstrating that carefully monitored RI-analysis is of significance. With crea-cut-offs of $<$ vs. $>1.3 \mathrm{mg} / \mathrm{dl}$ or within eGFR-subgroups of $>$ vs. $<90$ and $>$ vs. $<60 \mathrm{ml} / \mathrm{min}$, median PFS rates were not significantly different, demonstrating that despite RI, len was inducing responses and similarly effected PFS. Of note, also OS-rates with above crea-cut-offs did not reveal significant differences, as well with eGFR rates $>90$ vs. $<90$, median OS was not reached (nr) vs. 21.3 months $(\mathrm{ms} ; \mathrm{p}=0.0569)$ and with eGFR $>60$ vs. $<60 \mathrm{ml} / \mathrm{min} / 1.73 \mathrm{~m}^{2} \mathrm{nr}$ vs. $18.3 \mathrm{~ms}(\mathrm{p}=0.0571)$.

Conclusions: We demonstrate no significant PFS- or OS-differences within crea- and eGFR-subgroups with len-treatment, implying that the commonly perceived adverse prognostic impact of RI evades with incorporation of novel agents in MM.
Disclosure: Martina Kleber: Other Financial Relationships: Educational Grant Celgene

Monika Engelhardt: Other Financial Relationships: Educational Grant Celgene

\section{P820}

\section{Safety and tolerability of Lenalidomide (Revlimid) and low dose Dexamethason (Rd) in an unselected austrian Multiple Myeloma (MM) patient population. An analysis from the EUREM observational study}

Willenbacher, W. ${ }^{1}$; Keil, F.' ${ }^{2}$ Andel, J.' ${ }^{3}$ Lang, A. ${ }^{4}$; Farkas, M. ${ }^{5}$; Kretschmer, A. ${ }^{6}$; Sormann, S. ${ }^{7}$; Salamon, J. ${ }^{8}$; Pober, M. ${ }^{9}$

${ }^{1}$ Universitätsklinik Innsbruck, Innere V, Hämatologie \& Onkologie, Innsbruck, Austria, ${ }^{2}$ LKH Leoben, Innere Medizin, Dep. HämatologieOnkologie, Leoben, Austria, ${ }^{3}$ LKH Steyr, Innere Med. II, Steyr, Austria, ${ }^{4}$ LKH Rankweil/Feldkirch, Interne E, Rankweil, Austria, ${ }^{5}$ LKH Wiener Neustadt, Interne I, Wiener Neustadt, Austria, ${ }^{6}$ Onkologische Praxis, Waidhofen a.d. Thaya, Austria, ${ }^{7}$ Universitätsklinik für Innere Medizin Graz, Klinische Abteilung für Hämatologie, Graz, Austria, ${ }^{8} \mathrm{KH}$ Hietzing, 5. Medizin, Wien, Austria, ${ }^{9}$ LKH St.Pölten, Innere Med. I, St.Pölten, Austria

Introduction: $\mathrm{Rd}$ is an effective treatment for $\mathrm{MM}$ in advanced and early disease. $\mathrm{R}$ has been licensed by EMEA for second line therapy onwards and is recommended by several international guidelines (e.g. m-SMART). Because patient populations in clinical studies tend to be highly selected (e.g. less comorbidities, lower median age) we collected "real life" data on safety and tolerability of $\mathrm{Rd}$ in an unselected austrian patient population in 9 austrian cancer centers.

Methods: Data were prospectively acrrued by use of an anonymized 35 page $\mathrm{CRF}$, including data on comorbidities, risk factors for thrombosis, pre-treatment and myeloma response status, as well as extensive adverse event documentation. Data were independently monitored and transfered to an electronic data base.

Patient characteristics: From APR 2007 to NOV 200855 pts. (26 m., 29 f.) were recruited. Median age was 66.6 years. Immunoglobulin expression of MM was IgG (32 pts.), IgA (12 pts.), light-chain only (7 pts.) and unknown/ non-secretory (4 pts.). ECOG @ start of treatment was 2 or better in $82 \%$ of pts. Rd was applied as $1^{\text {st }}$ line $(9 \%), 2^{\text {nd }}$ line $(40 \%), 3^{\text {rd }}$ line $(24 \%)$ and beyond (27\%). Cytogenetic high risk features were found in $15 \%$ of pts. Prior treatments were Bortezomib-based regimes (38 pts.), Thalidomide-based (18 pts.), conventional cytostatic therapies (35 pts.) as well as autologous transplantation ( 9 pts.). $42 \%$ of pts. had pre-existing polyneuropathy, $20 \%$ creatinine clearances $<60 \mathrm{ml} / \mathrm{min}$. Established risk factors (rf) for thrombosis were frequent (103 in 55 pts.).

Results: (AEs given as \%/treatment cycles)

292 treatment cycles $(1-12$, median 5$)$ were analyzed. The leading toxicity was haematologic. Nadir reductions were $-0.85 \mathrm{~g} / \mathrm{dl}(\mathrm{Hb}),-100 \mathrm{G} / 1$ (thrombocytes) and

$-2450 / \mu \mathrm{l}$ (ANC). $3^{\circ}$ and $4^{\circ}$ cytopenias were rare $(2 \%)$. Other adverse events were infectious complications $(12 \%)$, thrombotic events $(3 \% ; 8$ episodes all non-fatal), cutaneous ( $1 \%)$, GI (3\%, one $4^{\circ}$ dysgeusia), polyneuropathy $(5 \%$; none worsening beyond $3^{\circ}$ ), fatigue $(5 \%)$, renal impairment $(1 \%)$, cardiac deterioration $(0.3 \%)$. No fatal adverse events were observed.

Conclusions: Rd proofed to be safe and well tolerated in an unselected "real life" , MM patient population. Toxicity was predominantly haematological and clinically well manageable. The severity and spectrum of other adverse events compared well to published results from phase III studies and will be analyzed in detail.

Disclosure: Wolfgang Willenbacher: Honoraria: Ja; Financing of Scientific Research: Ja

Michael Pober: No conflict of interest disclosed. 
P821

\section{High-dose chemotherapy and autologous blood stem cell transplantation in patients with multiple myeloma and the role of novel agents: A single centre experience}

\author{
Fenk, R. ${ }^{1}$; Neubauer, F. ${ }^{1}$; Bruns, I. ${ }^{1}$; Schröder, T. ${ }^{1}$; Saure, C. ${ }^{1}$; \\ Kondakci, M. ; Haas, R. ${ }^{1}$; Kobbe, G. ${ }^{1}$ \\ ${ }^{1}$ Heinrich-Heine-Universität Düsseldorf, Klinik für Hämatologie, Onkologie
} und klinische Immunologie, Düsseldorf, Germany

Introduction: High-dose chemotherapy (HDT) followed by an autologous blood stem cell transplantation (ASCT) has become the standard treatment for eligible patients with symptomatic multiple myeloma (MM).

Methods: We retrospectively analysed prognostic factors for the response and survival of 315 patients with MM who were transplanted in our centre between 1994 and 2008. At the time of diagnosis $53 \%, 27 \%$ and $15 \%$ of patients presented with ISS stage 1,2 and 3 and $3 \%, 15 \%$ and $81 \%$ with S+D stage I, I and III. At the time of autograft $5 \%$ of patients were in CR, $12 \%$ in vgPR and $49 \%$ showed a PR. All patients received peripheral stem cell support and high dose conditioning consisted of single melphalan 200 in $76 \%$ of patients while $24 \%$ were treated with different regimen. Maintenance treatment after HDT consisted of thalidomide in $34 \%$ of patients.

Results: Following HDT $19 \%$ of patients achieved a CR, 33\% a vgPR, 33\% a PR, $6 \%$ a MR or SD whereas 2\% showed PD. Median EFS and OAS from the time of transplant were 23 and 66 months, respectively. Transplant related mortality was $2.9 \%$. Results from univariate analysis showed, that achievement of a CR or vgPR after HDT and thalidomide maintenance treatment were associated with improved EFS and OAS. A LDH-level below $200 \mathrm{U} / 1$ and CRP-level of less than $0.6 \mathrm{mg} / \mathrm{dl}$ were further good prognostic factors for EFS, whereas a Hb-level of more than $10 \mathrm{mg} / \mathrm{dl}$, age younger than 60 years and ISS stage $1+2$ was associated with a prolonged OAS. Patients receiving thalidomide maintenance treatment had a median EFS and OAS of 30 and 76 months, respectively, which was significantly longer than $19(\mathrm{p}=0.0001)$ and $56(\mathrm{p}=$ 0.04 ) months in patients without thalidomide maintenance.

Looking at the prognostic influence of salvage therapies, we identified 215 patients with relapse after HDT. In univariate analysis thalidomide ( 25 versus 14 months, $\mathrm{p}=0.001$ ), bortezomib (26 versus 14 months, $\mathrm{p}=0.001)$, lenalidomide (46 versus 25 months, $\mathrm{p}=0.005$ ) and a second salvage HDT ( 30 versus 14 months, $\mathrm{p}=0.01$ ) were associated with a significantly prolonged OAS after posttransplantation relapse. Median OAS after relapse was 3, 16 and 37 months, respectively for patients who received $0,1-2$ or 3-4 of these treatment options ( $\mathrm{p}=0.001)$

Conclusion: HDT followed by ASCT is safe and effective. Thalidomide maintenance after HDT prolongs OAS. Thalidomide, bortezomib, lenalidomide an a salvage HDT independently prolonged OAS after posttransplantation relapse.

Disclosure: Roland Fenk: Advisory Role: Celgene; Honoraria: Celgene, Ortho Biotec; Financing of Scientific Research: Celgene

Guido Kobbe: Advisory Role: Celgene, Orhto Biotec; Honoraria: Celgene, Orhto Biotec; Financing of Scientific Research: Celgene
P822

\section{Incidence and risk factors of bisphosphonate induced osteonecrosis of the jaw (BONJ) in patients with multiple myeloma after HDT and ASCT}

\author{
Hörauf, N. ${ }^{1}$; Bumeder, I. ${ }^{1}$; Otto, S. ${ }^{2}$; Ebelt, K. ${ }^{1}$; Völkl, A. ${ }^{1}$ \\ Baumann, P. ${ }^{1}$; Schmidmaier, R. ${ }^{1}$; Alpay, N. ${ }^{1}$; Adam, C. ${ }^{1}$; \\ Röhnisch, T.1; Oduncu, F.S. ${ }^{1}$
}

${ }^{1}$ Klinikum der Universität München, Medizinische Klinik - Campus Innenstadt, Hämatologie und Onkologie, München, Germany, ${ }^{2}$ Klinikum der Universität München, Campus Innenstadt, Klinik und Poliklinik für Mund-, Kiefer- u. Gesichtschirurgie, München, Germany

Introduction: Osteonecrosis of the jaw (ONJ) is associated with the use of bisphosphonates in cancer patients. The aim of the present study was to analyse the incidence and risk factors of bisphosphonate induced osteonecrosis of the jaw (BONJ) in multiple myeloma (MM) patients after high-dose chemotherapy (HDT) and autologous stem cell transplantation (ASCT).

Methods: We retrospectively analysed the data of $120 \mathrm{MM}$ patients after HDT and ASCT to evaluate the incidence and risk factors of BONJ. For statistical analyses we used the SPSS program, the Fischer-Yates-test and the Mann-andWhitney-U-test. We compared the patient group with BONJ $(n=23)$ with the group without BONJ ( $\mathrm{n}=97)$.

Results: Twenty-three patients (19\%) developed BONJ. Six patients suffered several events of BONJ. Thus, the total incidence of BONJ events was $24 \%$. No significant impact on BONJ was observed for patient gender, disease stage, presence of osteolytic lesions, anaemia, beta- 2 microglobulin, osteoporosis, diabetes mellitus, and for renal failure. Neither did the type of tumor treatment affect the development of BONJ, such as the use of prednisone, dexamethason, melphalan, bortezomib, thalidomide, cyclophosphamid, bendamustin, lenalidomide, or the use of local radiation. However, there was a significant association with patients' current age $(\mathrm{p}=0,04)$, the type and total duration of bisphosphonate treatment $(\mathrm{p}=0,014)$, number of bisphosphonates' rotations $(\mathrm{p}=0,013)$, rheumatism $(\mathrm{p}=0,0001)$. Furthermore, dental manipulations were observed in $65,5 \%$ of BONJ events (19/29).

Conclusions: This is the first study to systematically analyse the incidence and risk factors of BONJ in MM patients undergoing HDT and ASCT. The use of bisphosphonates is highly associated with the occurrence of ONJ. Length of bisphosphonate exposure, type of bisphosphonate, rheumatism and previous dental procedures seem to be the most important risk factors.

Disclosure: No conflict of interest disclosed.

\section{P823}

Features of extramedullary multiple myeloma.

Soft tissue relapse as an evolving clinical dilemma in heavily pre- treated patients. A case series

\author{
Rasche, L. ${ }^{1}$; Bernard, C. ${ }^{1}$; Topp, M. ${ }^{1}$; Gattenlöhner, S. ${ }^{2}$; \\ Kapp, M. ${ }^{1}$; Einsele, H. $^{1}$; Knop, S. \\ ${ }^{1}$ Universitätsklinik Würzburg, Hämatologie/ Onkologie, Würzburg, \\ Germany, ${ }^{2}$ Universität Würzburg, Institut für Pathologie, Würzburg, \\ Germany
}

Introduction: Extramedullary (e) relapse in multiple myeloma (MM) is a serious event for MM patients that is encountered at increasing incidence. When eMM relapse occurs, no validated treatment options exist and most patients eventually die due to uncontrolled disease progression or therapyrelated toxicity. We therefore analyzed relapse patterns and individual treatment decisions of $24 \mathrm{eMM}$ cases at our institution to further elucidate this challenging phenomenon.

Material and method: We screened the myeloma registry of the University Hospital of Wuerzburg, Germany, for MM patients who developed eMM relapse during the course of their disease. From January 2007 to March 2010, 357 MM patients were treated at our institution, and 24 eMM relapses were identified. Results: Twenty-four $(6.7 \%)$ patients developed eMM relapse. Their median age was 56 (range, 41-79) years. Most frequently, eMM occurred at soft tissue sites $(67 \%)$ followed by parenchymal involvement $(25 \%)$ and malignant effusion $(12.5 \%)$. Interestingly, CNS involvement (meningiosis, cerebral masses) was observed in $21 \%$ of the patients. At diagnosis of eMM relapse, bone mar- 
row infiltration was absent in $46 \%$, in $21 \%$ infiltration was less than $20 \%$. Thus, over two-thirds had no or low bone marrow infiltration at the occurrence of eMM disease. In ten cases, biopsies from eMM lesions were available. The most striking finding was a dramatically increased proliferation index (Ki-67) of about $80 \%$ (range $40 \%-95 \%$ ) of all cancer cells.

EM relapse was treated with irradiation; dose-intense chemotherapy; novel agent-based therapies; and auto- allogeneic SCT concepts including DLI administration. 13/24 patients responded to therapy (1 CR, $12 \mathrm{PR}$ ), 1 patient had stable disease, 9 patients showed progressive disease or mixed response. Progression-free survival (PFS) was short with a median of 3 months (95\% CI: 1.61-4.39). Median overall survival (OAS) was 9 months (95\% CI: 4.9913.00). 11 patients survived for longer than six months, three patients longer than 12 months.

Conclusions: Extramedullary relapsed myeloma shows an aggressive growth pattern with short PFS and OAS. EMM can grow bone marrow-independently and shows CNS involvement in over $20 \%$. This is not compatible with the characteristics of an indolent lymphoma like MM usually is and, therefore, distinctive therapeutic approaches are urgently needed.

Disclosure: No conflict of interest disclosed.

\section{P824}

COMPARE - Results of a randomised study to assess the renal safety and efficacy of ibandronate and zoledronate in multiple myeloma patients Knauf, W. ${ }^{1}$; Bergner, R. ${ }^{2} ;$ Kropff, M. ${ }^{3}$; Poenisch, W. ${ }^{4}$; Kiewitz, C.
Goldschmidt, H. $^{6}$

${ }^{1}$ Onkologische Gemeinschaftspraxis am Bethanien-Krankenhaus, Frankfurt, Germany, ${ }^{2}$ Klinikum Ludwigshafen, Med. Klinik A, Ludwigshafen, Germany, ${ }^{3}$ Med. Klinik A der Universität, Münster, Germany, ${ }^{4}$ Med. Klinik II der Universität, Leipzig, Germany, ${ }^{5}$ Roche Pharma, Grenzach-Wyhlen, Germany, ${ }^{6}$ Med. Klinik V der Universität, Heidelberg, Germany

Background: Bisphosphonates (BPs) are widely used to prevent skeletal complications in patients (pts) with Multiple Myeloma (MM), which is characterised by osteolytic bone lesions or diffuse bone disease. As MM pts are at high risk for renal damage, renal safety may be a discriminator for selecting a particular BP.

Methods: COMPARE is a randomised, multi-centre, open-label, parallelgroup study to assess the renal safety of ibandronate (IBA) and zoledronate (ZOL) in pts with MM stage II-III (Salmon, Durie). Exclusion criteria included prior treatment with IBA or ZOL, serum creatinine $>4.0 \mathrm{mg} / \mathrm{dl}$, and estimated creatinine clearance (eCrCl; Cockcroft-Gault equation) $\leq 30 \mathrm{ml} / \mathrm{min}$. Pts were randomised to receive IBA $(6 \mathrm{mg})$ or ZOL $(4 \mathrm{mg})$, administered as IV infusion over 15 min Q4W for up to 92 weeks. ZOL dosage could be reduced at the physician's discretion. Primary endpoint was a relevant deterioration of renal function, defined as a decrease in $\mathrm{eCrCl}$ of $\geq 30 \%$ or to $\leq 30 \mathrm{ml} / \mathrm{min}$, respectively. Secondary endpoints included reductions of ZOL dose, number of, and time to first occurrence of, skeletal related events (SREs), and cases of osteonecrosis of the jaw (ONJ). Statistics were descriptive.

Results: 81 pts were randomised to receive IBA $(n=41)$ or ZOL $(n=40)$. The number of pts experiencing a relevant decrease in renal function was similar for treatment with IBA (6 pts, 14.6\%) and ZOL (5 pts, 12.5\%), respectively; however, 12 pts $(30 \%)$ in the ZOL group underwent at least one dose reduction. The mean treatment period was 52.6 and 44.3 weeks for IBA and ZOL treated pts, respectively (based on safety population). With IBA treatment, 9 pts $(22.0 \%)$ experienced an SRE compared to 12 SREs $(30.0 \%)$ reported in ZOL treated pts. Median time to first occurrence of an SRE was 393 days for IBA treatment and 244.5 days for ZOL treatment. ONJ was not observed in either treatment group.

Conclusions: In this randomised, multi-centre, open-label, parallel-group study comparing the renal safety and efficacy of IBA and ZOL in pts with stage II-III MM, the incidence of relevant decreases in estimated creatinine clearance was similar between treatment groups. With $30 \%$ of ZOL treated pts having had at least one dose reduction, physicians may have titrated ZOL dosing to achieve acceptable renal safety. SREs seemed to occur earlier and more frequently with ZOL compared to IBA treatment.
Sponsored by Roche Pharma AG, Grenzach, Germany. Protocol number: ML18508, www.roche-trials.com

Disclosure: Wolfgang Knauf: Honoraria: Roche Pharma AG; Financing of Scientific Research: Roche Pharma AG

Hartmut Goldschmidt: Advisory Role: Roche Pharma AG; Honoraria: Roche Pharma AG; Financing of Scientific Research: Roche Pharma AG, Novartis AG.

\section{P825}

\section{Schnitzler syndrome and our experience with anakinra (Kineret) therapy in two patients}

Szturz, P.1; Adam, Z. ";Čorbová, D. ${ }^{2}$; Neubauer, J. ${ }^{3}$; Prášek, J."; Koukalová, R. ${ }^{5}$; Řehák, Z.5 ${ }^{5}$ Mayer, J. ${ }^{1}$

${ }^{1}$ Faculty of Medicine of Masaryk University and University Hospital Brno, Department of Internal Medicine - Hematooncology, Brno, Czech Republic, ${ }^{2}$ Faculty of Medicine of Masaryk University and University Hospital Brno, Clinic of Dermato-venerology, Brno, Czech Republic, ${ }^{3}$ Faculty of Medicine of Masaryk University and University Hospital Brno, Radiological Clinic, Brno, Czech Republic, ${ }^{4}$ Faculty of Medicine of Masaryk University and University Hospital Brno, Clinic of Nuclear Medicine, Brno, Czech Republic, ${ }^{5}$ PET Centre at the Masaryk Memorial Cancer Institute, Department of Nuclear Medicine, Brno, Czech Republic

Schnitzler syndrome is a rare idiopathic disease characterized by chronic urtica, presence of monoclonal IgM immunoglobulin and further symptoms. This case report describes successful anakinra (Kineret) therapy in two patients followed up at our clinic. With the first patient the complaints started at the age of 45 , when dermal symptoms appeared in the form of intensely pruritic urticarial lesions the outbreaks of which were accompanied by intermittent fevers. Moreover, the patient suffered from pains in lumbar and pelvic regions. On a pelvic CT scan osteolytic-osteosclerotic skeletal changes were described and identified as hot spots on a scintigram. With the second patient the complaints started at the age of 44 and also urticarial rash was the case which was asymptomatic, non-pruritic. Later on however, severe attacks of shaking chills with bone and joint pains were added to during which new urticarial eruptions appeared. A CT scan of clavicles and pelvic bones showed hyperostosis. Due to a finding of monoclonal $\operatorname{IgM}$, both patients were sent to our clinic with suspected monoclonal gammopathy. After excluding other differential diagnoses, both the patients were diagnosed with Schnitzler syndrome. As to therapy, we used several medications (corticoids, 2-chlordeoxyadenosine, interferon alpha, cyclosporine, thalidomide, bortezomib) as well as the PUVA treatment but none of these put the disease into complete remission and we had to terminate the therapy after some time either due to insufficient effect of therapy or due to its adverse reactions. Only one medicament minimized the symptoms of Schnitzler syndrome in both patients with very good drug tolerance, namely anakinra (interleukin-1 receptor antagonist). In several hours after administering the first dose of the drug urticarial rash resolved completely. Bone pains and fever then subsided in several days along with normalization of blood inflammatory markers. The first patient has been on Kineret therapy for more than 30 months $(10 / 2007$ 4/2010) without any symptoms of Schnitzler syndrome. However, the anakinra therapy is permanent with the necessity of administering subcutaneous injections every day.

Disclosure: No conflict of interest disclosed. 


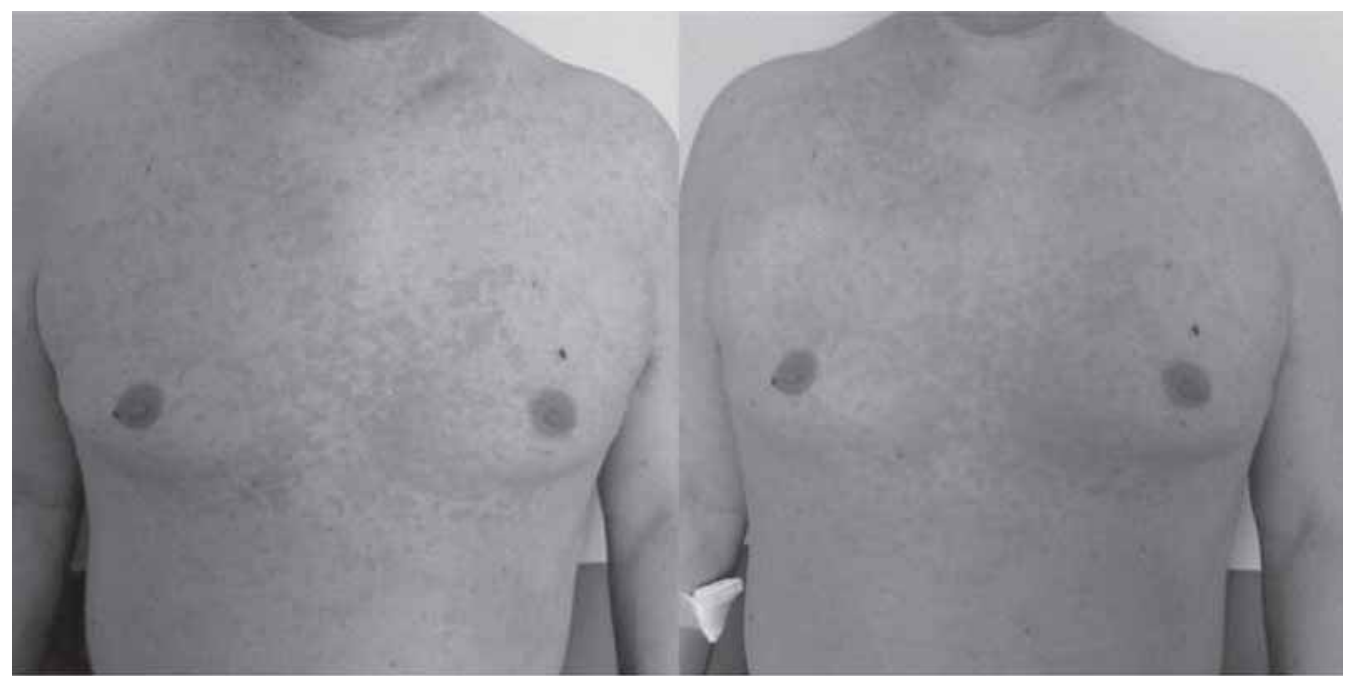

Prior to kineret

$2 \mathrm{~h}$ after application

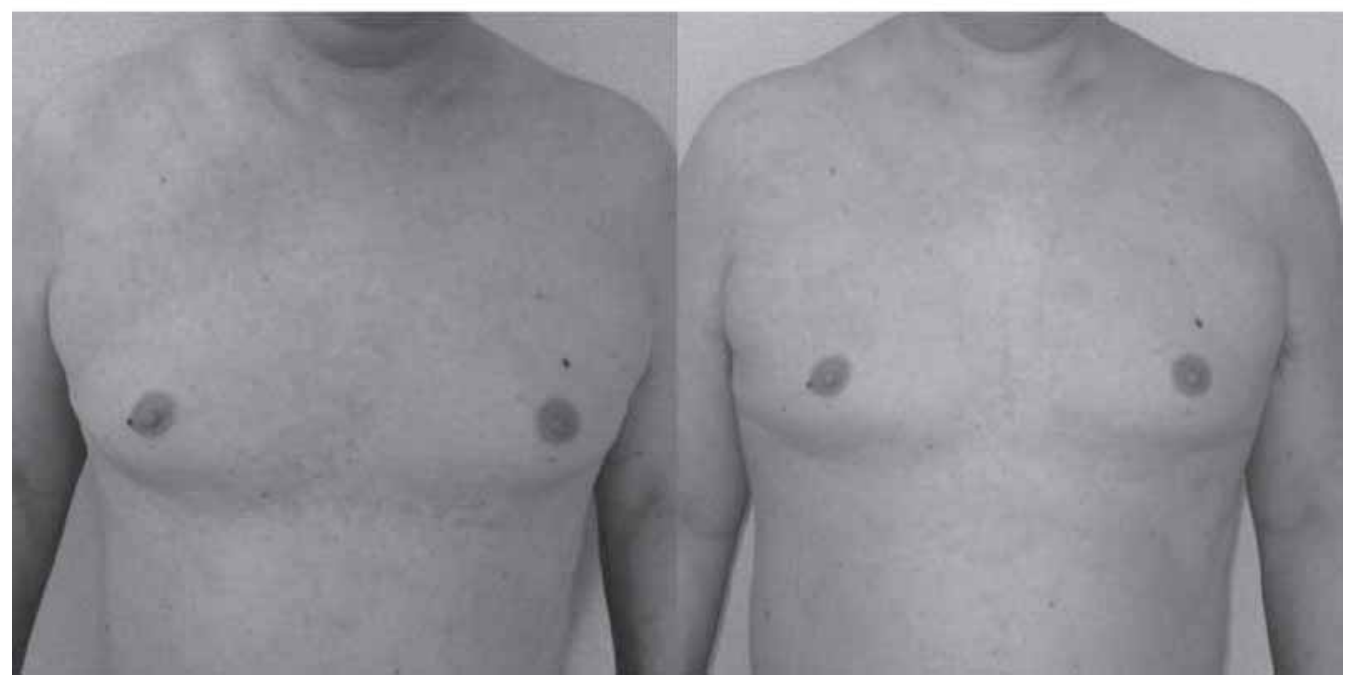

$6 \mathrm{~h}$ after application

$20 \mathrm{~h}$ after application

Fig. 1. Kineret therapy course

P826

\section{Acquired factor $X$ deficiency in a patient with amyloid light chain amyloidosis}

Distelmaier, L. ${ }^{1}$; Reumkens, M. ${ }^{2}$; Hettenkofer, T.2 ; MüllerBeißenhirtz, H. ${ }^{1}$; Noppeney, R. ${ }^{1}$; Zotz, R. ${ }^{3}$; Dührsen, U. ${ }^{1}$; Nückel, $H^{1}$; Böhm, S. ${ }^{2}$

${ }^{1}$ Universitätsklinik Essen, Klinik für Hämatologie, Essen, Germany, ${ }^{2}$ Katholische Kliniken Ruhrhalbinsel $\mathrm{GmbH}$, Klinik für Innere Medizin und Gastroenterologie, Essen, Germany, ${ }^{3}$ Centrum für

Blutgerinnungsstörungen und Transfusionsmedizin, Düsseldorf, Germany

Introduction: Systemic amyloid light chain (AL-) amyloidosis is a hematological disorder characterized by the deposition of amyloid fibrils, consisting of immunoglobin light chains, in organs and tissues.

The disease often leads to organ dysfunctions such as cardiac and renal insufficiency, polyneuropathy, hepatosplenomegaly and involvement of the gastrointestinal and respiratory tract. However, complications may include clinically significant hemorrhage or thrombosis.

Case report: Here we describe a 69-year old man who presented with a 4 months history of gastrointestinal bleeding, spontaneous bruising, weakness, dyspnoea and weight-loss.
The physical examination revealed multiple bruises, purpura and a slightly enlarged spleen.

Echocardiographic studies showed a reduced left ventricular function (EF $35 \%$ ) with a hypertrophy and mild dilatation without signs of a restrictive function disorder.

The patient's blood values showed a prolonged activated partial thromboplastin time $(50,6$ seconds) and reduced prothrombin time expressed as activity percentage (23\%), fibrinogen (440 mg/dl),a mild increase of the lambda light chains and a renal insufficiency stage I.

The coagulation disorder showed no response to treatment with prothrombin complex concentrates (PCC) and vitamin K. Further investigation revealed a factor X deficiency (5\%) with normal results for F II and F V. An inhibitor was excluded by mixing studies.

Despite of the elevated bleeding risk, a biopsy of the intestinal tract was performed which resulted in the diagnosis of AL-amyloidosis and we started treatment with a chemotherapy (Palladini regimen) consisting of melphalan and dexamethason.

Conclusion: Acquired factor X deficiency is an uncommon complication of amyloidosis and caused by adsorption of factor $\mathrm{X}$ by amyloid fibrils. It can lead to severe bleeding complications, not responding to treatment such as PCC, plasma exchange or vitamin K substitution. Splenectomy and high dose chemotherapy with autologous stem cell transplantation have been reported to be successful treatment options resulting in an increase of factor X-activity. Both strategies function through a temporary reduction of the amyloid fibrils 
and therefore a lower adsorption of factor X. In summary, acquired factor X deficiency is an uncommon but typical manifestation of amyloidosis. However, no effective therapy exists due to immediate adsorption of factor $\mathrm{X}$ by amyloid fibrils.

Disclosure: No conflict of interest disclosed.

\section{P827 \\ Longterm Lenalidomide therapy in relapsed or refractory multiple myeloma patients - a single-center experience}

\section{Denecke, B. ${ }^{1}$; Bross-Bach, U. ${ }^{1}$; Kanz, L. ${ }^{1}$; Weisel, K. ${ }^{1}$}

${ }^{1}$ Universitätsklinik Tübingen, Medizinische Klinik, Hämatologie, Onkologie, Immunologie, Rheumatologie, Pulmonologie, Tübingen, Germany

Introduction: Lenalidomide is an effective and well established treatment approved for relapsed or refractory myeloma disease. Although the substance has been introduced years ago, little is known about the longterm efficacy, possible specific side effects in longterm use and characteristics of the patient population profiting from prolonged use of lenalidomide.

Methods: We performed a retrospective analysis of all patients treated with lenalidomide at our institution since 2007 up to now. 74 patients with relapsed or refractory myeloma disease received lenalidomide in a dose of $25 \mathrm{mg}$ day 1-21 of a 28 day cycle, dexamethasone was added in a dose of $40 \mathrm{mg}$ day 1-4 9-12, 17-20. Dose adjustments were performed for toxicity and renal function. 12/74 patients received more than 12 months lenalidomide. Data were analyzed for treatment duration, response and possible side effects.

Results: In $12 / 74$ patients $(16.2 \%)$ treatment duration with lenalidomide and dexamethasone for more than 12 months was documented. Median age of this patient population was 69 years. Treatment duration ranged from 12 to 24 months with a median duration of 18 months. Lenalidomide was used in the median as the $3^{\text {rd }}$ line treatment (range $2-4$ ). 8/12 patients had previously undergone autologous stem cell transplantation (SCT), 1 patient received allogeneic SCT and 8 patients had been pretreated with bortezomib before start of lenalidomide treatment. Treatment resulted in PR in 10 patients, one patient each achieved CR and SD. Median time to PR was 35 days (range 30-135). Toxicity was mild and managable. Grade II/III fatigue was reported in 5 of 12 patients, which lead to a dose reduction in one patient. No grade III or IV neutropenia was documented. There were two cases of deep venous thromboses despite antithrombotic prophylaxis, both patients continued with lenalidomide treatment after initiation of full-dose anticoagulation. One grade III $^{\circ}$ infection occurred. No cases of new peripheral neuropathy were reported, pre-existing neuropathy did not worsen.

Conclusion: Lenalidomide is known to be a safe and highly effective treatment for multiple myeloma disease. Patients who tolerate long-term treatment show the combination of high treatment response and low toxicity. No unexpected adverse events in long-term use of lenalidomide were documented, and there were no prolonged cytopenias. We conclude that the treatment goal using lenalidomide should be the prolonged use $>12$ months.

Disclosure: Barbara Denecke: No conflict of interest disclosed.

Katja Weisel: Advisory Role: Celgene $\mathrm{GmbH}$; Honoraria: Celgene $\mathrm{GmbH}$; Financing of Scientific Research: Celgene $\mathrm{GmbH}$

\section{P828}

\section{Neurocognitive impairment in patients with multiple myeloma following chemotherapy}

Cenic, O. ${ }^{1}$; Schützl, P. ${ }^{1}$; Jank, R. ${ }^{1}$; Acel, A. ${ }^{2}$; Drach, J. ${ }^{2}$; Gisslinger, $H^{1}{ }^{1}$; Jaeger, U. ${ }^{1}$; Gaiger, $A{ }^{1}$

${ }^{1}$ Medical University of Vienna, Department of Internal Medicine I, Division of Hematology and Hemostaseology, Vienna, Austria, ${ }^{2}$ Medical University of Vienna, Department of Internal Medicine I, Division of Oncology, Vienna, Austria

Introduction: The introduction of new therapeutic compounds in the treatment of multiple myeloma has resulted in greater response rates, as well as improved progression free and overall survival. Questions regarding the impact of cancer treatment on the ability to perform tasks of our daily life, health related quality of life and neurocognitive functioning become of increasing relevance. Neurocognitive impairment ("chemobrain") is describing an altered state of cognition after chemotherapy affecting different domains of cognitive functioning, such as concentration, attention, memory, wordfinding, learning of new tasks, multitasking, etc. Reported prevalence rates of cognitive impairment after chemotherapy range between 16 and $75 \%$. We investigated the prevalence of neurocognitive impairment in 41 multiple myeloma (MM) patients treated at the $1^{\text {st }}$ Department of Internal Medicine, Medical University of Vienna.

Methods: To analyze different domains of cognitive functioning a computer based test system consisting of the „Determinationstest“ (DT), „Arbeitsleistungsserie“ (ALS) and „Wahrnehmungs- und Aufmerksamkeitsfunktionen“ (WAF) was used. All three tests were shown to be reliable and valid. Quality of life was assessed using EORTC QLQ-C30. Anxiety, depression and distress were determined by the Hosptital Anxiety and Depression Scale (HADS). Subjective perception of cognitive deficits was analyzed using the FEDA.

Results: $23(56 \%)$ of 41 patients showed test results below average $(T<40)$, indicative of neurocognitive impairment. Subjective perception of cognitive defects correlated only in 12 of 23 patients with the actual measured neuropsychological test results. Interim analysis showed no correlation between neurocognitive impairment and myeloma specific risk factors. Due to the heterogenity of myeloma treatment used, serial testing of cognitive functioning became necessary to analyze the impact of specific therapeutic agents on cognitive domains. These analyses are ongoing and final data will be presented at the Meeting.

Disclosure: Olivera Cenic: No conflict of interest disclosed.

Alexander Gaiger: Financing of Scientific Research: unrestricted research grant Celgene

\section{P829}

\section{Complete remission of multiple myeloma associated IgA pemphigus after therapy with bortezomib (Velcade), cyclophosphamide and dexamethasone: Case report}

Szturz, P.1 ; Adam, Z. ; Feit, J. ${ }^{2}$; Krejčí, M.1 ; Pour, L. ${ }^{1}$; Vašků, V. ${ }^{3}$; Čermáková, Z. ${ }^{4}$; Hájek, R. ${ }^{1}$; Mayer, J. ${ }^{1}$

${ }^{1}$ Faculty of Medicine of Masaryk University and University Hospital Brno, Department of Internal Medicine - Hematooncology, Brno, Czech Republic, ${ }^{2}$ Faculty of Medicine of Masaryk University and University Hospital Brno, Institute of Pathology, Brno, Czech Republic, ${ }^{3}$ Faculty of Medicine of Masaryk University and St. Anne's University Hospital Brno, 1st Department of Dermatology and Venereology, Brno, Czech Republic, ${ }^{4}$ University Hospital Brno, Department of Clinical Biochemistry and Hematology, Brno, Czech Republic

IgA pemphigus, similar to subcorneal pustulous dermatosis, is a rare complication of IgA type monoclonal gammopathy. This case report describes successful bortezomib therapy of $\operatorname{IgA}$ pemphigus associated with multiple myeloma. The first symptoms of the disease appeared in our patient, a woman born in 1940, at the age of 50 when vesiculobullous lesions began to form on her trunk and extremities. Due to a finding of monoclonal IgA lambda immunoglobulin in the serum, the patient was in 2001 sent to our clinic, where the diagnosis was closed as monoclonal gammopathy of unknown significance (MGUS) and IgA pemphigus. With regard to the activity of the dermal affliction, in 2003 the first immunosuppressive treatment was administered (cyclophosphamide $50 \mathrm{mg}$ daily for 6 months + dexamethasone $20 \mathrm{mg}$ on days $1-4$ and 15-18 in monthly cycles for 9 months). During this treatment intensity of the skin disorder ameliorated and monoclonal $\operatorname{IgA}$ levels decreased to nondetectable levels. However, after termination of the therapy bullous eruptions occurred again in their original intensity. Due to the early recurrence of skin symptoms, six rituximab $600 \mathrm{mg}$ infusions in weekly intervals were administered but without any lasting effect. In 2007 disease transformation into symptomatic multiple myeloma was identified, which required initiation of chemotherapy. First line anti-myeloma treatment (cyclophosphamide + adriamycine + dexamethasone - CAD) remained without any treatment response. Second line treatment (cyclophosfamide + thalidomide + dexamethasone CTD) significantly deteriorated dermal symptoms up to the clinical picture of erythrodermia. Only when third line treatment (cyclophosphamide $50 \mathrm{mg}$ p.o. daily, bortezomib 1,3 mg/sqm i.v. on days $1,4,8$ and 15 and dexamethasone $20 \mathrm{mg}$ p.o. on days 1-4 and 15-18 in 28-day cycles - CVD) had been given, 
rapid decline in monoclonal IgA concentration immediately after the $1^{\text {st }}$ cycle and negative immunofixation after 5 cycles were achieved. After the $1^{\text {st }}$ CVD cycle the skin lesions receded and from the $3^{\text {rd }}$ cycle on the patient has been completely without dermal symptoms. In total, 6 CVD cycles had been administered, which resulted in complete skin and haematological remission maintained for 18 months (from october 2008 to april 2010). Thus, in $\operatorname{IgA}$ pemphigus associated with monoclonal gammopathy of unknown significance transformed into multiple myeloma, the combined treatment with included bortezomib has definitely proved beneficial.

Disclosure: No conflict of interest disclosed.

P830

Ferritin- and iron-status- as well as impact of transfusionneeds on progression free- (PFS) and overall survival (OS) - analysis of their association to disease characteristics, organ function and comorbidities in multiple myeloma (MM) patients (pts)

Bayer, C. ${ }^{1}$; Ihorst, G. ${ }^{2}$; Kleber, M. ${ }^{1}$; Terhorst, M. ${ }^{1}$; de Pasquale, D. ${ }^{1}$; Daskalakis, M. ${ }^{3}$; Koch, B. ${ }^{4}$; Engelhardt, M. ${ }^{1}$

${ }^{1}$ Department of Hematology and Oncology, Freiburg, Germany, ${ }^{2}$ Department of Statistics and Biometry/Center of clinical trials, Freiburg, Germany, ${ }^{3}$ Department of Transfusion Medicine, Freiburg, Germany,

${ }^{4}$ Department of Laboratory Medicine, Freiburg, Germany

Introduction: Anemia is an eminent and relevant risk factor in MM. If anemia is induced by MM-progression, this leads to anti-MM-treatment, red blood cell (RBC) transfusions and infrequently erythropoetin treatment. Similarly, to the negative impact of iron overload (IO) in MDS and allogeneic transplantrecipients, IO in MM has also been postulated as a possible risk factor. The need to evaluate specific risk factors in MM and their impact on clinical routine is acknowledged worldwide, implying that novel and easily assessable risk factors are widely pursued.

Methods: We analysed a cohort of $n=91 \mathrm{MM}$ pts treated at our medical center between 1997-2009, assessing their pt characteristics, MM-treatment and known risk factors (such as cytogenetics, ß2-microglobulin [ß2-MG], LDH, creatinine-, estimated glomerular filtration rate [eGFR], CRP) and other parameters (e.g. proBNP), in association with iron-, ferritin-, transferrin-, transferrin saturation- (TS) levels and transfusion requirements $(\mathrm{RBC}+$ platelet transfusions [PT])

Results: Median iron-, ferritin-, transferrin- and TS-levels were $73 \mu \mathrm{g} / \mathrm{dl}$

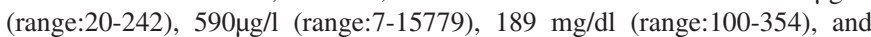
$27 \%$ (range:7-91), respectively. Median normal iron levels, associated with elevated ferritin- and low transferrin-levels, suggested either IO or MM-activity, the latter being reflected by stage III Durie \& Salmon- and ISS-disease in $98 \%$ and $44 \%$ of pts, respectively. Of interest, pts with ferritin levels $>590 \mu \mathrm{g} / \mathrm{dl}$ and $>1000 \mu \mathrm{g} / \mathrm{dl}$ revealed higher $32-\mathrm{MG}-$, LDH-, creatinine-, CRP- and pro-BNP-, and decreased eGFR-levels, suggesting more advanced MM with higher ferritin levels. This however, did not translate into different PFS and OS in various ferritin groups of $<100$ vs. $\geq 100,<500$ vs. $\geq 500,<1000$ vs. $\geq 1000,<$ 1500 vs. $\geq 1500$ and $<2000$ vs. $\geq 2000 \mu \mathrm{g} / \mathrm{dl}$. With TS-groups of $<\mathrm{vs}$. $\geq 45 \%$, PFS and OS were 33 vs. 28 months (ms) and 84 vs. 63ms, respectively, which -albeit not reaching significance- suggested that higher TS-rates -reflecting IO- lead to impaired PFS and OS. Median PFS and OS in our entire cohort was 31ms (range:27-49) and 79ms (56-116). Median RBC and PT transfusion rates were astoundingly high with $16(0-78)$ and $5(0-50)$.

Conclusions: Further analysis on the association of iron status, ferritin and IO with pt characteristics, cytogenetics, previous therapies, risks for and severity of infections, in regard to PFS and OS in this MM cohort is ongoing and will be presented at the meeting.

Disclosure: No conflict of interest disclosed.
P831

\section{Successful treatment of multiple myeloma patients and impaired renal function with lenalidomide - results of 4 german centers}

Oehrlein, K. ; Langer, C. ${ }^{2}$; Sturm, I. ${ }^{3}$; Pönisch, W. ${ }^{4}$ Kuhn, S. ${ }^{5}$; Hahn-Ast, C. ${ }^{6}$; Weisel, K.C. ${ }^{1}$

${ }^{1}$ Universitätsklinik Tübingen, Medizinische Klinik, Hämatologie, Onkologie, Immunologie, Rheumatologie, Pulmonologie, Tübingen, Germany, ${ }^{2}$ Universitätsklinik UIm, UIm, Germany, ${ }^{3}$ Charité - Campus VirchowKlinikum, Berlin, Germany, ${ }^{4}$ Universitätsklinik Leipzig, Leipzig, Germany, ${ }^{5}$ Celgene $\mathrm{GmbH}$, München, Germany, ${ }^{6}$ Universitätsklinik Bonn, Bonn, Germany

Introduction: About $50 \%$ of multiple myeloma patients demonstrate impaired renal function upon diagnosis. Of the patients that do not present with impaired renal function at diagnosis, an additional $25 \%$ will become renally impaired. Lenalidomide and dexamethasone is an effective and well tolerated treatment of relapsed or refractory multiple myeloma providing an overall response rate of $60 \%$. Dose modification according to the creatinine clearance $(\mathrm{CrCl})$ can be performed conveniently. However, there is limited data on the use of this regimen in myeloma patients with impaired renal function.

Methods: 19 patients of 4 german centers with impaired renal function and relapsed or refractory multiple myeloma were treated with lenalidomide/dexamethasone and retrospectively analysed. Lenalidomide was administered dose-adapted from day 1-21 of a 28 day cycle. Dexamethasone was given in all but 1 patient in a dosage determined by the local investigator. 3 patients received a combination treatment including cyclophosphamide.

Results: All 19 patients had renal function impairment at baseline defined by $\mathrm{CrCl}<60 \mathrm{ml} / \mathrm{min}$. 14 patients had a $\mathrm{CrCl}$ between 30-60 ml/min, 5 patients $<$ $30 \mathrm{ml} / \mathrm{min}, 4$ patients were permanently, 1 patient temporary dialysis dependent. Patients had a median of 2 pretreatments, 9 patients received autologous transplantation, 14 patients were pretreated with bortezomib. Median number of treatment cycles was 9.6 patients are still on drug. At least partial response was documented in $73.7 \% .42 .1 \%$ of patients showed temporary or permanent improvement of renal function measured by $\mathrm{CrCl}$. A median time of 28 days was documented until first response. Median progression free survival (PFS) was 16.8 months, median overall survival (OS) was not yet reached. Grade 3 or 4 leukopenia and thrombocytopenia occurred in $42.1 \%$ and $31.6 \%$, respectively. Four thrombotic events were documented, while all but one patient received thrombosis prophylaxis.

Conclusion: Lenalidomide and dexamethasone is highly effective and an attractive treatment option in multiple myeloma patients with impaired renal function. Toxicity is manageable. Hematologic toxicity may be increased when compared to data on patients with normal renal function. However, all grade 3 and 4 thrombopenias occurred in patients with pre-existing thrombocytopenia at baseline. Importantly, lenalidomide and dexamethasone can improve renal function in a substantial proportion of patients.

Disclosure: Katharina Oehrlein: No conflict of interest disclosed. Katja Weisel: Advisory Role: Celgene $\mathrm{GmbH}$; Honoraria: Celgene $\mathrm{GmbH}$; Financing of Scientific Research: Celgene GmbH

\section{P832 \\ Results from the Myeloma Registry of the University Hospital Graz}

Sormann, S. ${ }^{1}$; Bauer, F. ${ }^{1}$; Reitter, S. ${ }^{1}$; Pichler, M. ${ }^{1}$; Stranzl, T. ${ }^{1}$; Dekic, D. ${ }^{2}$; Linkesch, W.

${ }^{1}$ Medizinische Universität Graz, Klinische Abteilung für Hämatologie, Graz, Austria, ${ }^{2}$ Clinical Trials Management, Wien, Austria

Introduction: Myeloma has undergone rapid developments over the past years, with earlier treatment standards, such as MP or VAD, having become outdated and new drugs having emerged. The benefits of these new treatments need to be balanced against their potential harm.

Methods: The Myeloma Registry of the University Hospital Graz was established in January 2008 to determine the effectiveness and safety of myeloma treatments under real-life conditions. This analysis covers a period of 2 years and 112 patients cared for by the hospital's outpatient department, with initial 
diagnoses extending back to 1990. Statistical analyses were descriptive and exploratory in nature.

Results: Median age at the time of admission was 61.5 (28-87) years, and $52 / 112$ patients $(46 \%)$ were male. At the time of diagnosis, $36 / 112$ patients (32\%) had stage I disease, 16/112 patients (14\%) had stage II disease, and $60 / 112(54 \%)$ had stage III disease. $71 \%$ of patients presented as $\operatorname{IgG}, 21 \%$ as IgA, and $8 \%$ as other subtypes. The most common first-line therapy regimens were $\operatorname{PAD}(36 / 112,31 \%)$ and VAD $(28 / 112,27 \%)$. Overall, 43 patients received treatment regimens containing one of the 3 newer drugs for the treatment of multiple myeloma, i.e., bortezomib, lenalidomide, or thalidomide. 88/112 patients (79\%) received with biphosphonates. 17 patients (15\%) had not received any therapy by the end of the observation period. Autologous stem-cell transplantation (ASCT) was done in 52/112 patients (46\%), with 36 patients having undergone one, 9 patients 2 , and 7 patients 3 ASCTs. 5 patients underwent allogeneic transplantation. 49/112 patients (44\%) had side effects. The predominant treatment-related adverse reaction was peripheral polyneuropathy (PNP), occurring in 26 patients (23\%), 21 and 5 of whom had received bortezomib and thalidomide, respectively. The second most frequent adverse reaction was bisphosphonate-induced osteonecrosis, seen in 7 patients. Herpes zoster reactivation occurred in 8 , cytomegalovirus infection in 5 , and steroidinduced diabetes in 4 patients. Hematological and thrombotic events were rare.

Conclusions: Current treatment options for myeloma range from stem cell transplantation, well-established treatments, such as VAD, and the new drugs bortezomib, lenalidomide, and thalidomide. Besides the benefits of new therapy options, we are also seeing new side effects. PNP induced by bortezomib and thalidomide is documented as the major clinical problem in our myeloma registry.

Disclosure: No conflict of interest disclosed.

\section{P833}

\section{Comparison of hematopoietic recovery and supportive care requirements following autologous transplantation after tandem high-dose chemotherapy in multiple myeloma}

\section{Prasnikar, N. ${ }^{1}$; Movassaghi, K. ${ }^{2}$; Neubauer, A. ${ }^{3}$; Schwella, N. ${ }^{1}$} ${ }^{1}$ Department of Internal Medicine I, Community Hospital, Ludwigsburg, Germany, ${ }^{2}$ Department of Transfusion Medicine Humboldt University, Berlin, Germany, ${ }^{3}$ Department of Hematology/Oncology, Philipps University, Marburg, Germany

Purpose: We evaluated hematopoietic recovery with engraftment of white blood cells (WBC) and platelets (PLT) and supportive care requirements including transfusion of red blood cells (RBC) and single-donor platelet concentrates (PLTC), the length of antibiotical and antifungal treatment and of hospital stay in patients suffering from multiple myeloma (MM) who received tandem high-dose chemotherapy (HDCT) followed by autologous stem cell transplantation (ASCT).

Methods: Forty-four patients (19 women, 25 men) underwent tandem HDCT/ ASCT for MM treatment. We determined the time of engraftment concerning WBC and PLT as well as the number of transfused RBC and PLTC, the number of patient-days on intravenous (i. v.) antibiotical and i. v. antifungal treatment, and the length of hospital stay.

Results: Patients with a median age of 55 years (range: $38-65$ ) at the time of diagnosis received predominantly Vincristin, Adriamycin and Dexamethasone (VAD) as induction chemotherapy (72\%). The median period of time from diagnosis to peripheral blood stem cell mobilization was 6 months $(1,2-52)$. Mobilization was conducted with cyclophosphamide (CPM) at a median dose of $2,0 \mathrm{~g} / \mathrm{m}^{2}(0,4-4,0)$ plus granulocyte colony-stimulating factor. Leukapheresis started in median after 12 days (9-18) after CPM and a median dose of $8,8 \times 10^{6}$ $\mathrm{CD} 34+$ cells/ $/ \mathrm{kg}\left(3,1-34,0 \times 10^{6}\right)$ was collected. Following the first cycle of HDCT a median dose of $5,2 \times 10^{6} \mathrm{CD} 34+$ cells $/ \mathrm{kg}\left(2,5-25,0 \times 10^{6}\right)$ was reinfused and after the second course of HDCT a median dose of 4,6×10 $\mathrm{CD} 34+$ cells $/ \mathrm{kg}\left(1,0-17,4 \times 10^{6}\right)$ was reinjected. There were no significant differences between the first and second HDCT/ASCT course concerning engraftment of WBC with a median of 13 days (8-24) versus (vs.) 12 days (9-39) and of PLT with a median of 13 days (8-26) vs. 14 days (8-22). The median number of transfused RBC, 2 units $(0-8)$ vs. 0 units $(0-12)$, and the median number of transfused PLTC, 1 unit (0-11) vs. 1 unit (0-12) were not significantly different between the both courses of HDCT/ASCT. Median days on i. v. antibiotics, 8 days (0-26) vs. 7 days (0-47), and i. v. antifungals, 19 days (5-30) vs. 20 days (10-35) and the median length of hospital stay, 21 days (16-30) vs. 21 days (12-47), were not significantly different between both groups.

Conclusion: The comparison of hematopoietic recovery, supportive care requirements and hospital stay shows no significant differences between the first and the second course of HDCT/ASCT in MM patients.

Disclosure: No conflict of interest disclosed.

\section{P834}

Prognostic scoring system for multiple myeloma patients undergoing high-dose therapy with autologous stem cell transplantation

\section{Kordelas, L. ${ }^{1}$; Liebisch, P.2; Beelen, D.W. ${ }^{1}$; Dührsen, U. ${ }^{3}$;} Nückel, $H^{3}{ }^{3}$

${ }^{1}$ Universitätsklinik Essen, KMT-Klinik, Essen, Germany, ${ }^{2} \mathrm{Ev}$. BethesdaJohanniter-Krankenhaus, Klinik für Hämatologie und Onkologie, Duisburg, Germany, ${ }^{3}$ Universitätsklinik Essen, Klinik für Hämatologie, Essen, Germany

Introduction: Patients (pts.) with multiple myeloma (MM) are historically staged according to Salmon and Durie with respect to hemoglobin $(\mathrm{Hb})$ and calcium $(\mathrm{Ca})$ levels, the presence or absence of osteolytic lesions, the paraprotein level and the urinary light chain excretion. This staging system led to a more uniform patient classification and improved comparability in study design. In 2005 the International Staging System (ISS) was published by the International Myeloma Working Group. However, both classification systems do not consider the new cytogenetic markers and the effect of high dose chemotherapy. We therefore have identified a number of standard parameters which can predict overall survival (OS) and event-free survival (EFS) quite accurately in a homogenously treated cohort of more than 100 newly diagnosed pts. with MM treated with high-dose melphalan followed by autologous stem cell transplantation.

Methods: We have established a database of 104 pts. with MM who were treated one $(n=43)$ or two times $(n=61)$ with high-dose melphalan followed by autologous stem cell transplantation. Median age of pts. was 59.1 years (range 28 - 73). We have identified a number of parameters which in a univariate analysis are significant with respect to OS: $\mathrm{Hb}$ level $<10.0 \mathrm{mg} / \mathrm{dl}$ ( $\mathrm{p}=0.03$ ), creatinine $>1.3 \mathrm{mg} / \mathrm{dl}(\mathrm{p}=0.02)$, ISS 2 or $3(\mathrm{p}=0.05)$ and cytogenetic high-risk abnormalities (i.e. deletion of chromosome band $17 \mathrm{p} 13$ or translocation $\mathrm{t}(4 ; 14)(\mathrm{p} 16.3 ; \mathrm{q} 32), \mathrm{p}<0.0001)$. Synthesizing these parameters into a scoring system we have analysed our pts. database and awarded for each item (e.g. Hb $<10.0 \mathrm{mg} / \mathrm{dl}$ ) one point, so the pts. could collect 0 to 5 points (ISS 2 resulted in $1 / 2$, ISS 3 in one point). Hence, we received one group of 64 pts. who have a score of $0,0.5$ or 1.0 , and a second group of 40 pts. who have a score of 1.5 or higher.

Results: The two groups differ significantly in OS (log rank test $\mathrm{p}=0.001)$. The median OS in group 1 was 2,573 days and 1,413 days in group 2. The EFS equally reaches statistical significance: median EFS of 853 days in group 1 and 762 days in group $2(\mathrm{p}=0.05)$.

Conclusion: Our data suggest that a simple, yet powerful prognostic score can be established by analysing a limited number of easily available MM parameters, like $\mathrm{Hb}$ and creatinine levels, ISS and cytogenetics to predict the probability of OS and EFS for patients undergoing high-dose therapy with autologous stem cell transplantation quite accurately.

Disclosure: No conflict of interest disclosed. 
P835

\section{Pericardial effusion - a rare side effect of treatment with lenalidomide}

\author{
Hager, V.'; Austein, T.'; Schultens, A. ${ }^{2}$; Groenhagen, B. ${ }^{1}$; \\ Kohlhoff, $E^{2}$ \\ ${ }^{1}$ St. Bernhard Hospital, Medizinische Klinik II, Hämatologie und \\ Internistische Onkologie, Brake, Germany, ${ }^{2}$ St. Bernhard Hospital, \\ Medizinische Klinik I, Allgemeine Innere Medizin, Brake, Germany
}

Introduction: Lenalidomide (Revlimid $®$ ) is an immunmodulator with antiangiogenetic and -neoplastic effects. It is used in the treatment of multiple myeloma or the MDS on detection of 5-q-deletion. Side effects are neutro-/ thrombopenia, obstipation, asthenia, fatique, venous thromboembolism, pleural effusions and edema.To our knowledge we present the first report on another very rare and potentially lethal side effect.

Case report: In Janurary 2010 a 38-year old woman with multiple myeloma (Stage IIIB at first diagnosis 10/2007) was referred to our haematological ambulance with dyspnoe and edema. The initial treatment in 2007 consisted of an autogenic stem-cell transplant. Because relapse occured one year later an allogenic stem-cell transplantation was performed. In summer 2009 a further relapse was diagnosed, thus therapy with thalidomide was initiated. Due to PNP therapy was switched to lenalidomide. By the time of presentation the patient had been treated with lenalidomide $(25 \mathrm{mg} /$ day $)$ and dexamethasone (40mg/week) for two months.

The X-ray displayed signs of cardial dilatation and pleural effusions.

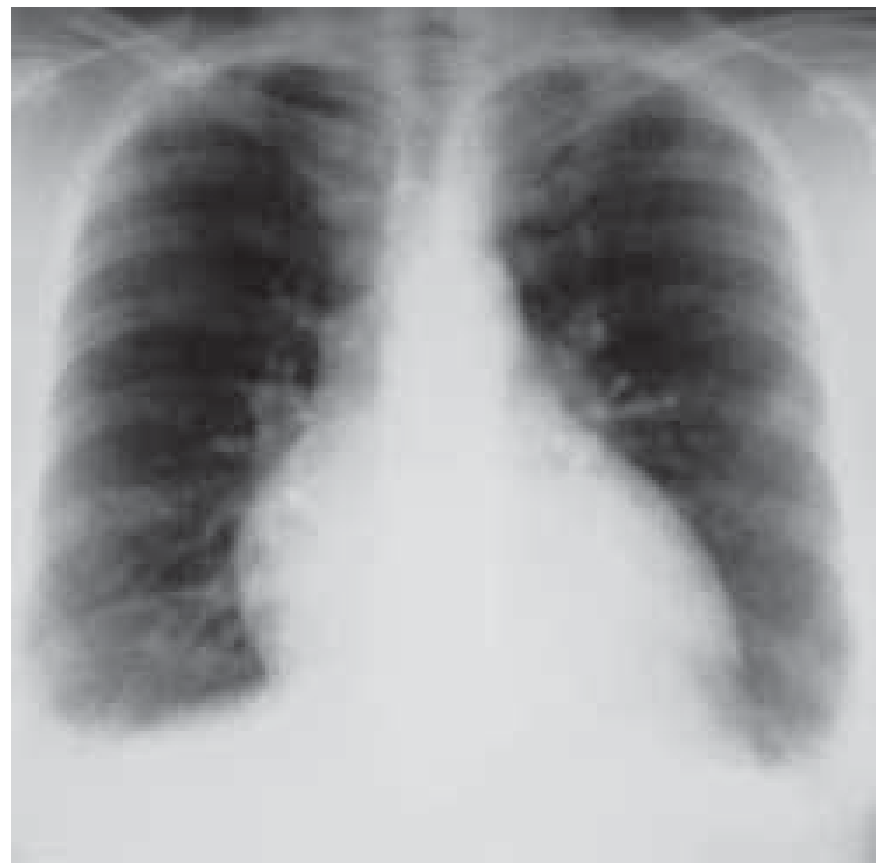

Fig. 1. After two months therapy with lenalidomide.

Echocardiography revealed a haemodynamically comprimising pericardial effusion. There were no indications for amylodosis, kollagenosis, viral ,bacterial, myo- or pericarditis. Four weeks after treatment with lenalidomide was suspended and furosemide therapy was initiated the effusions dissipated.

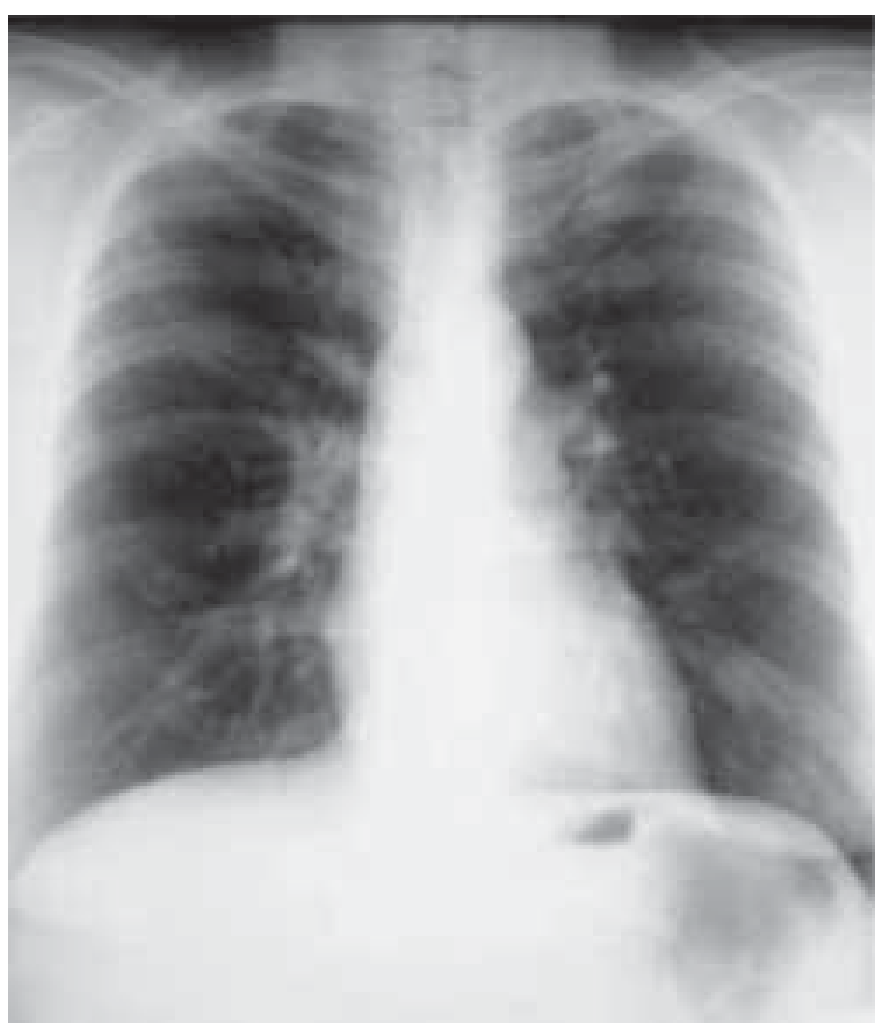

Fig. 2. 4 weeks after lenalid. therapy was stopped.

No effusions developed under continuation of therapy with a lower dose of lenalidomide (10mg/day) and dexamethasone (40mg/week) .

Conclusions: To our knowledge pericardial effusions as side effect of lenalidomide has not been described thus far. If dyspnoe and edema occur under treatment pericardial effusions should be considered. As diagnostic tests echocardiography or x-ray should be sought early to prompt treatment.

Disclosure: No conflict of interest disclosed.

P836

\section{Bendamustin - a novel therapeutic option in relapsed/ refractory multiple myeloma}

\section{Krieger, O. ; Machherndl-Spandl, S. ; Binder, M.1; Weltermann, A. $^{1}$; Kasparu, $\mathrm{H}^{1}{ }^{1}$}

${ }^{1} \mathrm{KH}$ der Elisabethinen, 1. Interne Abteilung, Linz, Austria

Background: The prognosis of patients with multiple myeloma (MM) has improved because of new drugs. The hybrid-alkylating agent Bendamustin (in combination with corticosteroids) has been tested in first line treament of MM, but there are little data about the therapy in relapsed (rel) or refractory (ref) patients.

Patients and methods: 15 patients $(11 \mathrm{w} / 5 \mathrm{~m})$ with rel/ref MM were treated with Bendamustine between January 2008 und April 2009. Bendamustine was given at a dosage of $70-90 \mathrm{mg} / \mathrm{m}^{2}$ day $1+2$ in combination with corticosteroids. The age of the patients was 65 (49-78) years, the duration of disease 56 (11-117) months and lines of prior therapy were 4 (2-9); 11 patients had a relapse after autologous, 1 after allogeneic transplantation. 6 patients were cytopenic at start of Bendamustine.

Results: In 15 patients 73 (1-14/pat.) cycles were administered. A reduction of paraproteinaemia occured in 9 patients $(60 \%)$ and in $4 / 9$ more than $50 \%$ of the first level. In 3 patients (20\%) the disease was stabilized, only 2 patients $(13 \%)$ had a refractory disease and in 1 patient the treatment had to be stopped because of toxicity (Diarrhoe grade 3 ) after the first cycle. The maximal response was observed after $3(2-7)$ cycles. The median time to therapy failure was $6(3-17)$ months. Severe infections due to grade 4 haematotoxicity were the main side effect. $4 / 5$ patients died due to septicaemia/pneumonia. Non haematologic side effects were rare (1x Diarrhoe grade 3, 1x hypertension 
grade $2,1 x$ sec. malignancy - breast carcinoma). We could not observe a worsening of neuropathy.

Conclusion: Bendamustine (in combination with corticosteroids) could be an effective strategy in rel./ref MM, but the main problem is infection due to severe haematotoxicity. Prospective randomized trials with new drugs are warranted.

Disclosure: No conflict of interest disclosed.

\section{P837}

\section{Bortezomib resistance in three patients with plasma cell leukemia}

Radkowski, R. ${ }^{1}$; Asemissen, A.M. ${ }^{1}$; Rottinghaus, I. ${ }^{1}$; Heinsch, M. ${ }^{1}$; Aristoteles, G. ${ }^{1} ;$ Aul, C. $^{1}$

${ }^{1}$ St. Johannes Hospital, Duisburg, Germany

Introduction: The modern era of myeloma therapy is marked by the availability of multiple highly effective therapeutic agents. The proteasome inhibitor (BOR) has been successfully included in current antimyeloma regimes, but little is known about activity in plasma cell leukaemia (PCL) which is characterized by high numbers of circulating plasma cells ( $\left.>2 \times 10^{\wedge} 9 / 1\right)$, lack of CD56 expression, higher incidence of unfavourable cytogenetics and an aggressive clinical course.

Methods: We report the outcome of BOR-based therapies in three patients with PCL (primary PCL $n=2$, secondary PCL $n=1$ ). One patient received BOR plus liposomal doxorubicin as primary treatment, combination therapy with $\mathrm{BOR}$ and dexamethason after only one cycle VAD, and one patient received treatment with BOR after he had failed two previous lines in combination chemotherapy. All patients were treated with BOR at a dosage of $1.3 \mathrm{mg} / \mathrm{m}^{2}$ on days $1,4,8$, and 11 of a 21 days-cycle for up to 6 courses. Responses were graded according to IMWG criteria (Durie et al, 2006).

Results: Despite temporary reductions in M-protein serum concentrations, none of the patients fulfilled the criteria of partial remission or stable disease. All patients stopped treatment with BOR because of progressive disease characterized by increasing serum paraprotein levels, growing medullary plasma cell infiltration and/or deteriorating bone destruction. All patients achieved partial remission following salvage polychemotherapy with cyclophosphamid/ anthracyclines or TCED.

Conclusion: These results suggest lacking efficacy of BOR in primary and secondary PCL. In all Patients resistance to BOR was overcome by conventional combination chemotherapy. Our experience is in marked contrast to previous studies reporting response rates up to $92 \%$ in small series of PCL patients (Musto et al, 2007). The reasons for the resistance to BOR in PCL are unknown, but are likely due to differences in the genetic programs between extramedullary and medullary clonal plasma cells.

Disclosure: No conflict of interest disclosed.

\section{Posterdiskussion}

Lymphome

P838

Post-transplantation lymphoproliferative diseases (PTLD) in paediatric and juvenile patients - a cohort of 125 cases

Hussein, K. ${ }^{1}$; Maecker-Kolhoff, B. ${ }^{2}$; Klein, C. ${ }^{2}$; Kreipe, H.H. ${ }^{1}$

${ }^{1}$ Medizinische Hochschule Hannover, Institut für Pathologie, Hannover, Germany, ${ }^{2}$ Medizinische Hochschule Hannover, Klinik für Pädiatrische Hämatologie und Onkologie, Hannover, Germany

Introduction: Post-transplantation lymphoproliferative diseases (PTLD) are mainly Epstein-Barr-virus (EBV)-associated disorders. Paediatric and juvenile patients have a higher risk, because children are more often EBV-naïve before transplantation and have their primary infection under immunosuppressive therapy.

Methods: Retrospective evaluation of 125 juvenile PTLD-cases $(1 \propto: 1.3 \AA$; median age 2 years/range 1 month -16 years).

Results:

1. Transplanted organs: liver (34\%), kidney (34\%), heart (24\%), and other organs $(8 \%)$
2. Duration between transplantation and PTLD-manifestation: median 33 months, range 2-174 months.

3. Most frequent extranodal PTLD-localisation: tonsils $(42 / 125,34 \%)$ and gastrointestinal tract $(27 / 125,22 \%)$

4.1 Early lesions, $n=48 / 125(38 \%)$.

4.1.1 Lymphatic hyperplasia, $\mathrm{n}=37 / 125(29 \%)$.

4.1.2 Infectious mononucleosis-like lesions, $\mathrm{n}=11 / 125(9 \%)$.

4.2 Polymorphic PTLD, $\mathrm{n}=21 / 125(17 \%)$.

4.3 Monomorphic PTLD, $\mathrm{n}=56 / 125$ (45\%).

4.3.1 Most frequent monomorphic PTLD entities were high-grade large B-cell lymphomas $(n=37 / 56,66 \%)$, Burkitt-lymphomas $(n=8 / 56,14 \%)$ and Hodgkin-lymphomas $(n=5 / 56,9 \%)$.

Early onset manifestation of PTLD within $<12$ months (median 6 months, range 2-11 months) comprised mainly large B-cell lymphomas ( $n=20 / 125$, $16 \% / \mathrm{n}=20 / 56,36 \%$, respectively) but rarely polymorphic PTLD $(\mathrm{n}=3 / 125$, $2 \%)$ or early lesions $(n=6 / 125,4 \%)$.

4.3.2 Rare entities: lymphomatoid granulomatosis Liebow/angiocentric B-cell lymphoma in the host-lung $(n=1)$, extramedullary anaplastic plasmacytoma in the oral cavity $(\mathrm{n}=1)$, and EBV-positive nodular T-cell lymphoma ( $\mathrm{n}=$ 1).

Conclusions: This large cohort of paediatric and juvenile PTLD shows that $\mathrm{i}$ ) monomorphic PTLD/lymphomas are frequently B-cell PTLD, ii) PTLD frequently involve extra-nodal sides such as tonsils and the gastrointestinal tract and iii) early onset PTLD are frequently large B-cell lymphomas.

Disclosure: No conflict of interest disclosed.

\section{P839}

\section{Detection of free immunoglobulin light chains in cerebrospinal fluids of patients with central nervous system lymphomas}

Baraniskin, A. ${ }^{1}$; Heute, C. ${ }^{1}$; Kuhnhenn, J. ${ }^{1}$; Alekseyev, A. ${ }^{1}$; Schmiegel, W. ${ }^{1}$; Schlegel, U. ${ }^{1}$; Pels, H. ${ }^{1}$; Schroers, R. ${ }^{1}$

${ }^{1}$ Ruhr-Universität Bochum, Knappschaftskrankenhaus Bochum Hämatologie und Onkologie, Bochum, Germany

Diagnosis of central nervous system (CNS) lymphoma depends on histopathology of brain biopsies, since no reliable disease marker in the cerebrospinal fluid (CSF) has been identified yet. B-cell lymphomas such as CNS lymphomas are clonally restricted and express either kappa or lambda light chains. The aim of this study was to find out a potential diagnostic value of free immunoglobulin light chains potentially released into the CSF of CNS lymphoma patients. Kappa (к) and lambda $(\lambda)$ free light chains (FLC) were measured in CSF and serum samples collected from 21 patients with primary and secondary CNS lymphomas and 14 control patients with different neurological disorders. FLC concentrations and ratios were compared between patient groups, and further analyzed in correlation with clinical, cytopathological, and radiological findings. FLC concentrations for all patients were lower in CSF as compared to serum. In CNS lymphoma patients, the FLC ratios in CSF were significantly higher (range 392 to 0.3 ) compared to control patients (range 3.0 to 0.3 ). Irrespective of cytopathological proven lymphomatous meningitis, in 11/21 lymphoma CSF samples the FLC ratios were markedly above 3.0 indicating a clonally restricted B-cell population. Increased FLC ratios in CSF were found in those patients showing subependymal lymphoma contact as detected in magnetic resonance imaging. In summary, this is the first report demonstrating that a significant proportion of patients with CNS lymphomas display a markedly increased FLC ratio in the CSF.

Disclosure: Alexander Baraniskin: No conflict of interest disclosed Roland Schroers: Financing of Scientific Research: Die Reagenzien für die Bestimmung der Freien Leichtketten i.R. des Projektes wurden von der Firma FreeLite finanziert. 
P840

\section{Intraperitoneal and intrapleural application of Rituximab in refractory CD20-positive B-cell lymphoma}

Christoph, S. ${ }^{1}$; Sellmann, L. ${ }^{2}$; Röth, A. ${ }^{2}$; Elmaagacli, A.H. ${ }^{1}$; Beelen, D.W. ${ }^{1}$; Dührsen, U. ${ }^{2}$

${ }^{1}$ Universität Duisburg-Essen, Klinik für Knochenmarktransplantation, Essen, Germany, ${ }^{2}$ Universität Duisburg-Essen, Klinik für Hämatologie, Essen, Germany

Introduction: Rituximab is a standard component of the treatment of CD20positive B-cell Non-Hodgkin lymphoma (NHL). Its efficacy and safety have been established in a variety of studies. Routinely, rituximab is given intravenously, but other routes of administration have also been described. Pleural effusions or ascites in lymphoma patients refractory to systemic treatment can be clinically challenging. Intrapleural or intraperitoneal application of rituximab might be a therapeutic alternative, but published experience is scant.

Patients and methods: Five patients with pleural effusion and/or ascites due to refractory low-grade NHL were treated. Mean age was 69 years. In four patients, intrapleural or intraperitoneal rituximab was administered concomitantly with systemic chemotherapy. In one case rituximab was given alone. For intrapleural therapy, the pleural cavity was drained and rituximab was instilled weekly for four doses at a dose of $400 \mathrm{mg}$ in $100 \mathrm{ml} 5 \%$ glucose. For intraperitoneal treatment, ascites was drained and rituximab was instilled every other day for four doses at a dose of $375 \mathrm{mg} / \mathrm{m}^{2}$ in $250 \mathrm{ml} 5 \%$ glucose. Patients received standard premedication, and instillation time was 5 minutes.

Results: In one case rituximab was given intraperitoneally followed after several weeks by intrapleural administration. Two patients were treated with a single application of rituximab via the intraperitoneal route, and two patients received a single dose via the intrapleural route. A sustained regression of ascites and/or pleural effusion was observed in three out of five patients. Adverse events were negligible. In two patients, response could not be evaluated due to death from rapid systemic lymphoma progression.

Conclusions: Intrapleural and intraperitoneal administration of rituximab is well tolerated without local complications or systemic adverse events. Three out of five patients responded completely, and pleural effusion or ascites did not recur. Rituximab is an effective treatment option in refractory pleural effusions or ascites caused by CD20-positive lymphoma.

Disclosure: Sandra Christoph: No conflict of interest disclosed.

Ulrich Dührsen: Advisory Role: Roche Pharma AG; Honoraria: Roche Pharma AG; Financing of Scientific Research: Roche Pharma AG

\section{P841}

\section{Replacement of conventional Doxorubicin by liposomal Doxorubicin is a safe and effective alternative in treatment of NHL-patients with cardiac risk factors}

\section{Schmitt, C.J. ${ }^{1}$; Dietrich, S. ${ }^{1}$; Ho, A.D. ${ }^{1}$; Witzens-Harig, M. ${ }^{1}$ ${ }^{1}$ Universität Heidelberg, Medizinische Klinik V, Heidelberg, Germany}

Anthracyclin is a major component in the treatment of Non Hodgkin`s Lymphoma (NHL). Due to it's cardiac toxicity potential curative and palliative treatment is often limited in patients with pre-existing cardiac dysfunction Several published analyses allude a improved cardiac tolerability of liposomal doxorubicin as compared to conventional doxorubicin. In the current study we retrospectivly analysed efficacy and toxicity of therapy with liposomal doxorubicin on cardiac function monitored by echocardiography pre- and post therapy in 21 patients with established diagnosis of NHL and impaired left ventricular ejection fraction or pre-existing cardiac risk factors. Patients were diagnosed with aggressive B cell lymphoma ( $\mathrm{n}=14)$, indolent $\mathrm{B}$ cell lymphoma $(\mathrm{n}=5)$ or T-cell lymphoma $(\mathrm{n}=2)$ and were treated with liposomal doxorubicin as constituent of CHOP-regimen. 17 patients were treated in first line therapy, 4 of 21 had a relapsed disease. Lymphoma stage I and II was present in 6 patients and stage III and IV in 5 and 10 patients respectively. A median number of 3,3 cycles was administered with a median dose of $34 \mathrm{mg} / \mathrm{m}^{2}$. Median age was 68 years, median IPI was 3 , in 13 of 21 an elevated LDH was present. The LVEF was assessed by echocardiography pre- and post chemotherapy. Among the 21 patients enrolled we found significant change in left ventricular ejection fraction with finally lethal acute cardiac decompensation in only one patient with severe congenitale heart failure. Among the 20 patients analysed for their remission state overall and complete response rate were $85 \%$ and 40 $\%$ respectively. 2 years Event Free Survival and Overall Survival were 58\%. 7 cases of Grade III-IV haematological toxicity were observed as well as 4 episodes of neutropenic fever leading to hospitalisation. No infection related death occured. $25 \%$ of patients developed a hand-foot-syndrom leading to discontinuation of treatment, whereas in this study development of a handfoot-syndrom seems to correlate positively with the applicated peak dose. Taken together we conclude that replacing doxorubicin with liposomal formulations in polychemotherapy regimen such as CHOP could be an efficient alternative in treatment of patients with preexisting cardiac dysfunction. Because of the high rates of hand-foot-syndrome during treatment with pegylated liposomal doxorubicin further studies should be launched to evaluate a rationale for the use of unpegylated formulations.

Disclosure: No conflict of interest disclosed.

\section{P842}

A randomized, double-blind, placebo-controlled study to assess the effectivity and safety of IL-6 inhibition by Siltuximab (CNTO-328) in patients with multicentric Castleman's disease

Fingerle-Rowson, G. ${ }^{1}$; Vermeulen, J. ${ }^{2}$; Qi, M. ${ }^{3}$; Safer, K. ${ }^{4}$; Godwin, A. ${ }^{5}$; Prince, H.M. ${ }^{6}$; Rossi, J.F.7; Cornfeld, M. ${ }^{8}$; van Rhee, $F^{9}$

1Janssen-Cilag $\mathrm{GmbH}$, Medical Affairs, Neuss, Germany, ${ }^{2}$ CentoCor Europe, Leiden, Netherlands, ${ }^{3}$ OrthoBiotech Oncology Research and Development, Leiden, Netherlands, ${ }^{4}$ OrthoBiotech Oncology Research and Development, Malvern, United States, ${ }^{5}$ Herring Clinical Consulting Inc., Wayne, United States, ${ }^{6}$ Cabrini Hospital, Malvern, Australia, ${ }^{7}$ University Hospital Chu Lapeyronie, Montpellier, France, ${ }^{8}$ CentoCor OrthoBiotech Onc, Raritan, United States, ${ }^{9}$ University of Arkansas for Medical Sciences, Little Rock, United States

Introduction: Multicentric Castleman's disease (MCD) is a rare disease in which patients (pts) develop benign lymphadenopathy, hepato- and/or splenomegaly and B-symptoms such as fever, weight loss or fatigue. Advances in understanding the biological basis of MCD have led to the identification of interleukin (IL)-6 as a critical mediator of disease progression. Optimal standard therapies have not been established for MCD and currently there is no approved systemic treatment modality. Since treatments based on experience in Non-Hodgkin-Lymphoma (NHL) do not deliver satisfactory results, novel approaches such as the inhibition of IL-6 are medically warranted for MCD pts.

Siltuximab is a chimeric monoclonal antibody which binds human IL-6 with high affinity and specificity and prevents its interaction with the IL6-receptor (IL-6R). In an ongoing phase I study, neutralization of IL-6 activity by Siltuximab has led to a high objective tumor response rate $(52 \%)$ and clinical benefit rate $(78 \%)$ in subjects with MCD with a favorable safety profile ${ }^{1}$. These results have prompted a trial to definitely assess the efficacy and safety of Siltuximab in combination with best supportive care (BSC) versus placebo + BSC.

Methods: Subjects with symptomatic and measurable MCD are eligible if they are HIV-/HHV-8-negative. Pts with skin lesions only, prior lymphoma or prior exposure to IL-6 / IL-6R targeted therapy are excluded.

78 pts will be randomly assigned in a 1:2 ratio to placebo + BSC or Siltuximab + BSC. Placebo or Siltuximab $(11 \mathrm{mg} / \mathrm{kg})$ will be administered as an $1 \mathrm{~h}-\mathrm{IV}$ infusion every 3 weeks for up to 4 years or until disease progression. The primary objective is to evaluate durable tumor and symptom response in the intent to treat-population. Secondary objectives include additional efficacy measures, safety, patient reported outcomes, and pharmacologic assessment. Status: This is the very first, worldwide, randomized trial for pts with MCD which attempts to define a new evidence-based treatment approach based on IL-6 inhibition. The extreme rarity of MCD makes it difficult for physicians to define a standardized treatment approach and poses unique challenges regarding the conduct of this clinical trial to pharmaceutical companies. Thus, the traditional center-based approach to pts recruitment will be abandonned and replaced by a network and community based awareness approach. The worldwide study is now open for enrolment in Germany. Reference: ${ }^{1}$ van Rhee et al., Blood 2008, \# 1008 
Disclosure: Günter Fingerle-Rowson: Employment or Leadership Position: Janssen-Cilag

F. van Rhee: Advisory Role: CentoCor; Honoraria: CentoCor

\section{P843}

Is persistent polyclonal B cell lymphocytosis a premalignant state for a clonal B cell lymphoma?

Nebe, C.T. ${ }^{1}$; Atta, J. ${ }^{2}$; Hensel, M. ${ }^{3}$; Baldus, M. ${ }^{4}$; Chow, K.-U. ${ }^{2}$ ${ }^{1}$ Onkologikum Frankfurt, Frankfurt am Main, Germany, ${ }^{2}$ Ambulantes Krebszentrum Schaubstrasse, Frankfurt am Main, Germany, ${ }^{3}$ Mannheimer Onkologie Praxis, Mannheim, Germany, ${ }^{4}$ Onkologie Praxis, Rüsselsheim, Germany

Polyclonal B cell lymphocytosis (PPBL) was initially described in 1982 and is a rare condition with chronic, stable, persistent and polyclonal lymphocytosis, with the presence of binucleated lymphocytes in the peripheral blood and a polyclonal increase in serum immunoglobulin-M (IgM). In 2009 Cornet and coworkers (Leukemia. $2009 \mathrm{Feb}$;23(2):419-22) reported for the first time in the liteature on the long term follow up of 111 patients of this apparently benign entity. They showed that PPBL was associated with recurrent chromosomal abnormalities and a typical cytogenetic profile including isochromosome $3 \mathrm{q},+\mathrm{i}(3 \mathrm{q})$, premature chromosome condensation (PCC), both abnormalities in the same patient or chromosomal instability. PPBL was diagnosed in this study in 91 women $(82 \%)$ (median age 40 years, range 19-66 years) and 20 men (median 41 years, range 28-57). The patients, smokers in $98 \%$ of cases, were either asymptomatic or had minor and nonspecific complaints, such as fatigue. Physical examination was usually within normal limits, except for a mild splenomegaly present in $10 \%$ of cases.

We detected in our laboratory three cases of PPBL all of them with an imbalance of light chain restriciton in the CD5+ and CD5- B cells in a way that each of the two subpopulation had a mutual restriction to kappa or lambda. In a fourth case submitted as an kappa IgG MGUS suspicious for transformation into myeloma we found a mild B cell lymphocytosis with $50 \%$ kappa and lambda where the lambda were CD5+CD20(+)CD43+ and the kappa CD5CD20++CD38+CD43-. Lymphocyte morphology was a mixture of small CLL cells and immunoblasts. Immunophenotype and morphology taken together with cytoplasmic light chains gave diagnosis of lymphoplasmocytic lymphoma LPL. In the bone marow from the same patient requested for myeloma diagnosis the same B cell immunophenotype was observed in part with plasma cell differentiation $(\mathrm{CD} 138+38+)$. BM morphology was a mixture of small CLL cells, few immunoblasts and plasma cells.

In summary PPBL is an underdiagnosed condition in smokers where immunopenotyping adds to its diagnosis beside thorough morphologic examination of the peripheral blood film. The relationship between PPBL, light chain disequilibrium and LPL warrants further multicentric investigations. Furthermore, persistant leukocytosis in smokers should be scrutinized for lymphocytosis and then for PPBL to obtain a better estimate for the frequency of PPBL in smokers that might be underestimated at the moment.

Disclosure: No conflict of interest disclosed.

\section{P844}

Unusual EBV- and HHV-8 associated posttransplant lymphoproliferative disorders other than classic PTLD

Jöhrens, K. ${ }^{1}$; Aczigöz, Ö. ${ }^{2}$; Neuhaus, R. ${ }^{3}$; Hartwigsen, D. ${ }^{4}$; Dörken, B. ${ }^{2}$; Riess, H. $^{2}$; Anagnostopoulos, I. ${ }^{1}$; Trappe, R. ${ }^{1}$, Deutsche Studiengruppe für Posttransplantationslymphome (DPTLDSG)

${ }^{1}$ Institut für Pathologie, Charité Campus Mitte, Berlin, Germany, ${ }^{2}$ Medizinische Klinik m. S. Hämatologie/Onkologie, CVK, Charité Universitätsmedizin Berlin, Berlin, Germany, ${ }^{3}$ Klinik für Allgemein-, Visceral- und Transplantationschirurgie CVK, Charité-Universitätsmedizin Berlin, Berlin, Germany, ${ }^{4}$ Medizinische Klinik 1, Malteser Hospital St. Franziskus, Flensburg, Germany

Epstein-Barr virus (EBV) and human herpesvirus type 8 (HHV-8) share the ability of infecting lymphocytes and inducing a spectrum of lymphoproliferations. Here we describe two patients with solid organ transplantation who developed peculiar lymphoproliferations associated with an infection of both viruses. The lymph node of the first patient showed a focal Kaposi's sarcoma manifestation and morphological features of the plasma cell type of Castleman disease. In contrast to published findings the plasmablasts and plasma cells within the follicular mantle zones exhibited a polytypic expression of the immunoglobulin light chains with a predominance of the lambda-positive cells. A low number of plasma cells expressed the latent nuclear antigen (LANA)-1 of HHV-8, while additional in situ hybridization for EBERs revealed several positive cells morphologically corresponding to plasmablasts and occasional plasma cells. The lymph node of the second patient showed morphological and immunophenotypical features of a germinotropic lymphoprolifeative disorder. All plasmablasts within the follicular structures coexpressed LANA-1 and EBER transcripts. The presence of a coinfection with EBV and HHV-8 in lymphoproliferations after solid organ transplantation seems to be a rare phenomenon. Analysis of cases with uncommon histological features for the presence of both viruses is, however, indicated.

Disclosure: No conflict of interest disclosed.

\section{P845}

\section{Molecular characterization of chromosomal alterations in} T-cell neoplasms

Grabarczyk, P.1; Keding, D. ${ }^{1}$; Przybylski, G. ${ }^{1}$; Braun, F.1; Grönick, J. ${ }^{1}$; Dittmann, K. ${ }^{1}$; Assmus, K. ${ }^{1}$; Schmidt, C.A. ${ }^{1}$

${ }^{1}$ Mokulare Hämatologie, Greifswald, Germany

Dysregulation of genes due to chromosomal aberrations plays a key role in malignant transformation. Translocations are routinely found in cancer cells using cytotogenetic methods, but only few of them have been further characterized on the molecular level due to time and labour consuming genomic cloning methods. To better understand the pathogenesis of malignant diseases novel rapid high throughput approaches for determination of the sequence of chromosomal breakpoints are needed. In this study combination of fine tiling comparative genomic hybridization (FT-CGH) and ligation mediated PCR (LM-PCR) was used for cloning and molecular characterization of novel chromosomal breakpoint regions and gene rearrangements in T-cell neoplasms. A custom fine-tiling oligonucleotide array of 385,000 oligonucleotides (NimbleGen) covering $24 \mathrm{Mb}$ of different genomic areas was designed for comparative genomic hybridization $(\mathrm{CGH})$. DNA losses or gains were further analyzed by LM-PCR.

Results: FT-CGH analysis revealed several mono- and biallelic DNA losses. Using LM-PCR with sets of nested primers located at the borders to the deleted regions amplification products were obtained differing from the germline control. Genes identified at breakpoint sites included interestingly a serine-phospatase (PPP) involved in the RAF-MAPK-ERK signal cascade. Further, a demethylase was found reteatedly deleted in T-cell neoplasia. Functional analysis including siRNA strategies were performed in T-cells to unravel biologic effects of these alterations. Genes identified and functional data obtained will be presented.

Summary / conclusions: We have shown that the combination of FT-CGH and LM-PCR allows the amplification and molecular characterization of gene rearrangements and chromosomal translocation in patient samples and may unravel genes relevant for malignant ttrasnformation.

Disclosure: No conflict of interest disclosed.

\section{P846}

\section{The role of BCL11B in T-cell neoplasia survival and proliferation}

Grabarczyk, P. ${ }^{1}$; Nähse, V. ${ }^{1}$; Delin, M. ${ }^{1}$; Przybylski, G. ${ }^{1}$; Depke, M. ${ }^{2}$; Hildebrandt, P. ${ }^{2}$; Völker, U. ${ }^{2}$; Schmidt, C.A. ${ }^{1}$

${ }^{1}$ Universitätsklinikum Greifswald, Molekulare Hämatologie, Greifswald, Germany, ${ }^{2}$ Interfaculty Institute for Genetics and Functional Genomics, Greifswald, Germany

Background: BCL11B expression was identified in T-cells, neurons, keratinocytes and recently in a subset of squamous cell carcinomas. Depletion of this gene resulted in reduction of cellular survival in immature primary cells 
and cell lines accompanied by replication stress and increased sensitivity to DNA damage. The ectopic overexpression in HeLa cells and a hematopietic progenitor cell line caused cell cycle retardation of unknown origin. These features predispose BCL11B to function as an oncogene or a tumor suppressor. Genetic losses and inactivating mutations found in T-cell lymphomas in mice supported the tumor suppressive role. The amplifications and expression of wild-type BCL11B shown in human T-cell leukemias/lymphomas indicated its oncogenic potential. In order to better understand the role of BCL11B in the survival of human T-cell derived tumors we analyzed the effects of BCL11B accumulation on cell survival and cell cycle progression.

Materials and methods: A retroviral-vector based BCL11B overexpression system was developed in Jurkat and huT-78 T-cell lines. The BCL11B deletion mutants were created using PCR. The influence of elevated Bcl11b on survival was assayed by treatment with radiomimetic drugs and TRAIL followed by Annexin- $\mathrm{V}$ binding assay. The DNA damage was measured by $\gamma-\mathrm{H} 2 \mathrm{AX}$ detection. Cell cycle was monitored using propidium iodide staining and BrdUincorporation assay.

Results: BCL11B overexpression lead to increased resistance to radiomimetic drugs without affecting the death-receptor apoptotic pathway. This effect was accompanied by an accumulation of cells at G1 associated with upregulation of CDKN1C (p57) and CDKN2C (p18) cyclin dependent kinase inhibitors Moreover, p27 and p130 proteins were accumulated and the SKP2 gene responsible for their degradation was repressed. The expression of MYCN oncogene was silenced and resulted in significant depletion of the protein. The cell cycle delay and DNA-damage induced apoptosis resistance coincided anc were dependent on the presence of the histone deacetylase binding $\mathrm{N}$-terminal domain.

Conclusions: The data presented here support a potential role of BCL11B in promoting tumor survival and chemoresistance and encourages us to develop BCL11B-inhibitory strategies as potential therapeutic approaches.

Disclosure: No conflict of interest disclosed.

\section{P847}

\section{Peripheral blood CD3- CD4+ T-cells: A useful diagnostic tool in angioimmunoblastic T-cell lymphoma (AITL)}

Singh, A. ${ }^{1}$; Schabath, R. ${ }^{2}$; Flörcken, A. ${ }^{1}$; van Lessen, A. ${ }^{1}$; Dörken, B. ${ }^{1}$; Ludwig, W.-D. ${ }^{2}$; Pezzutto, A. ${ }^{1}$; Westermann, J. ${ }^{1}$

${ }^{1}$ Charité - Campus Virchow-Klinikum, Medizinische Klinik m.S.

Hämatologie/Onkologie, Berlin, Germany, ${ }^{2} \mathrm{HELIOS}$ Klinikum Berlin-Buch,

Klinik für Hämatologie, Onkologie und Tumorimmunologie, Berlin,

Germany

Introduction: Angioimmunoblastic T-cell lymphoma (AITL) belongs to the subgroup of mature T cell lymphoma according to WHO and is one of the common T-cell lymphomas in Western countries. Diagnosis can be challenging in many cases. Particularly in cases in which histological confirmation cannot be easily achieved, immunophenotyping of peripheral blood can give important clues for the differential diagnosis of AITL.

Methods: We have previously shown that CD3- CD4+ T-cells in peripheral blood are a characteristic immunophenotypic finding in AITL patients. In this study the frequency of CD4- CD3+ T-cells was compared in AITL patients and in patients with other leukemic CD4+ T-cell lymphomas as confirmed by histology. 16 patients with leukemic AITL were compared with 24 patients with other CD4+-T cell lymphomas . Immunophenotyping of peripheral blood by flow cytometry was performed in a lymphocyte gate using fluorochromelabeled antibodies against CD3, CD2, CD4, CD5, CD7, CD8, CD14, CD16, CD19, CD56, CD57 and TCR.

Results: In 16/16 AITL patients a small but distinct population of CD3-CD4+ T-cells was found (mean percentage of CD3-CD4+ T cells in the lymphocyte gate: $12,0 \pm 3,97 \%$, range $0,1-51,8 \%$ ). In contrast, CD3-CD4+ T-cells could be detected in only $2 / 24$ patients with other leukemic CD4+ T-cell lymphomas (one patient with mycosis fungoides, one patient with peripheral T-NHL not otherwise specified). The frequency (\% lymphocytes) in these cases was 0,58 $\%$ and $10,3 \%$ respectively. Further immunophenotypic analyses showed that the aberrant T cells in AITL also express pan T-cell markers such as CD2, CD5 and partially CD7. Surface TCR expression could not be detected.

Conclusion: Our comparative study shows that flow cytometric detection of CD3-CD4+ T-cells in peripheral blood is a characteristic feature of AITL. In our cohort of patients with leukemic CD4+ T-cell lymphoma the detection of
CD3-CD4+ T cells had a positive predictive value for the diagnosis "AITL" of $89 \%$. Therefore, immunophenotyping by flow cytometry is particulary useful in the differential diagnosis of AITL, even if the aberrant T-cell population has a very low frequency. Since AITL is mostly CD3+ on immunohistochemistry we conclude that CD3-CD4+ T-cells do not represent the major population of neoplastic cells. More likely they can be considered as a small but characteristic subpopulation in AITL. Further biological characterization of this subfraction of lymphoma cells is warranted.

Disclosure: No conflict of interest disclosed.

\section{P848}

\section{Derepression of an endogenous long terminal repeat activates the CSF1R proto-oncogene in human lymphoma}

Mathas, S. ${ }^{1,2}$; Lamprecht, B. ${ }^{1}$; Walter, K. ${ }^{3}$; Kreher, S. ${ }^{4}$ Kumar, R. ${ }^{5}$, Hummel, M. ${ }^{6}$; Lenze, D. ${ }^{6}$; Köchert, K. ${ }^{1}$; Bouhlel, M. ${ }^{3}$; Richter, J. ${ }^{7}$; Soler, E. ${ }^{8}$; Stadhouders, R. ${ }^{8}$; Jöhrens, K. $^{6}$; Wurster, K. ${ }^{1}$; Callen, D. ${ }^{5}$ : Harte, M. ${ }^{9}$; Giefing, M. ${ }^{7}$; Barlow, R. ${ }^{3}$; Stein, H. $^{6}$; Anagnostopoulos, I. ${ }^{6}$; Janz, M. ${ }^{1}$; Cockerill, P. ${ }^{3}$; Siebert, R. ${ }^{7}$; Dörken, B. ${ }^{1}$; Bonifer, C. ${ }^{3}$

${ }^{1}$ Charité - Campus Virchow-Klinikum, Berlin, Germany, ${ }^{2}$ Max-DelbrückCenter for Molecular Medicine, Berlin, Germany, ${ }^{3}$ Leeds Institute of Molecular Medicine, Leeds, United Kingdom, ${ }^{4}$ Charite, Campus Virchow Klinikum, Berlin, Germany, ${ }^{5}$ University of Adelaide, Adelaide, Australia, ${ }^{6}$ Charité - Campus Benjamin Franklin, Berlin, Germany, ${ }^{7}$ ChristianAlbrechts-University Kiel \& University Hospital Schleswig-Holstein, Kiel, Germany, ${ }^{8}$ Erasmus MC, Rotterdam, Netherlands, ${ }^{9}$ Cytopia Research pty Ltd, Victoria, Australia

Introduction: It was the aim of our study to analyze mechanisms and consequences of lineage-inappropriate gene expression, a phenomenon which is in particular well established in certain hematopoietic malignancies and might be involved in their pathogenesis and progression. One of the most prominent examples of reprogramming of the normal gene expression pattern among human hematopoietic malignancies occurs in classical Hodgkin lymphoma (HL). HL is a common lymphoma that usually originates from mature B cells, but the malignant Hodgkin-/Reed-Sternberg (HRS) cells have largely lost expression of B cell-specific genes. This includes lack of the B cell receptor (BCR) expression, which is normally required for B cell survival, suggesting that the survival of HRS cell is regulated by different means.

Methods: RNA preparation, DNA preparation, semiquantitative and quantitative PCR analyses, Western blotting, electrophoretic mobility shift assay, reporter assays, analysis of growth and apoptosis, DNase I hypersensitive site mapping, bisulfite pyrosequencing, immunohistochemistry, immunoprecipitation.

Results: The malignant HRS cells of HL depend on the non-B, myeloid-specific proto-oncogene colony-stimulating factor 1 (CSF1R). We show that inhibition of CSF1R activity induces growth arrest and cell death of HRS cell lines. Furthermore, we demonstrate that CSF1R transcription in HRS cells initiates at an aberrantly activated endogenous LTR of the MaLR family (THE1B). Derepression of the THE1 subfamily of MaLR LTRs is widespread in the genome of Hodgkin lymphoma cells and is associated with impaired epigenetic control due to loss of expression of the corepressor CBFA2T3 in HRS cell lines and primary HRS cells

Conclusion: These data suggest that the upregulation of non-B lineage genes, which are normally silenced in B cells, is required for HRS growth and survival, and might thus provide alterative survival pathways following loss of BCR activity. Furthermore, our data show that LTR derepression is involved in the pathogenesis of human lymphomas, a finding which might have diagnostic, prognostic and therapeutic implications.

Disclosure: No conflict of interest disclosed. 
P849

Inhibition of aberrant Notch activity in Hodgkin lymphoma cells leads to downregulation of alternative NF- $\mathrm{KB}$ signaling and induction of apoptosis

Schwarzer, R. ${ }^{1}$; Dörken, B. ${ }^{1,2}$; Jundt, F. ${ }^{1,2}$

${ }^{1}$ Charité - Campus Virchow-Klinikum, Hämatologie/Onkologie, Berlin, Germany, ${ }^{2}$ Max-Delbrück-Center for Molecular Medicine, Berlin, Germany

Aberrant activity of Notch1 and constitutively activated NF- $\mathrm{KB}$ signaling are hallmarks of Hodgkin lymphoma (HL) cells. We have shown earlier that activation of the classical NF- $\mathrm{kB}$ pathway in HL cells is associated with mutations in the main NF-кB inhibitor ІкBa and with constitutive activity of ІкB kinases (IKKs). Furthermore, we could demonstrate that NF- $\mathrm{KB}$ activity in HL cells protects them from apoptosis and is necessary for tumor growth in vivo. Here, we analyzed the consequences of specific Notch inhibition on cell growth and NF- $\kappa \mathrm{B}$ activation in HL cells using a $\gamma$-secretase inhibitor (GSI). GSImediated downregulation of Notch signaling blocked proliferation and induced apoptosis, indicating constitutive Notch activity in HL cells that is necessary for their survival. Analysis of NF-кB activity by reporter assays revealed concomitant reduction of transcriptional NF- $\mathrm{KB}$ activity upon GSI treatment. We then analyzed whether NF-kB protein levels were affected by Notch inhibition. Intriguingly, only the levels of nuclear p52 and RelB, effectors of the alternative NF-кB pathway, dropped dramatically. This finding was supported by strongly reduced DNA binding of these NF- $\mathrm{KB}$ subunits. Consistent with the notion that Notch signaling acts on the non-canonical NF- $\mathrm{KB}$ pathway, phosphorylation of the p52 precursor p100, which is indispensable for the activation of the alternative branch of NF- $\mathrm{KB}$ signaling, dropped dramatically upon treatment in GSI-sensitive HL cell lines, while it remained unchanged in GSI-resistant L428 cells. Thus, we provide evidence that aberrant Notch activity is essential for HL cell growth and survival and show for the first time that Notch activates non-canonical NF-кB signaling in HL cells.

Disclosure: No conflict of interest disclosed.

\section{P850}

\section{Reactive oxygen species (ROS) communicate both apoptosis and target protein release in bortezomib-based chemotherapy of $\mathrm{HL}$}

Hansen, H. ${ }^{1}$; Vahdat, A. ${ }^{1}$; Engert, A. ${ }^{1}$; Pogge von Strandmann, E. ${ }^{1}$ ${ }^{1}$ Universitätsklinik Köln, Med I, Köln, Germany

Introduction: A hallmark of Hodgkin lymphoma (HL) is the constitutive activation of the NF-kB which leads to apoptosis resistance in the malignant HRS cells. Small molecules that influence this survival pathway are currently investigated in the clinic. We investigated the cytotoxicity and mechanisms of cell death of the proteasome inhibitor bortezomib in Hodgkin lymphoma cell lines.

Methods: Membrane protein expression, apoptosis and reactive oxygen species (ROS) were measured by flow cytometry and Western blot. Ectodomain shedding and particle release was separated by ultracentrifugation and analyzed by Western blot and ELISA technique.

Results: Proteasome inhibition by bortezomib caused elevation of ROS and concomitantly stimulated shedding of CD30, CD138 and interleukin-6 receptor, later followed apoptosis. Receptor down-regulation and release of the ectodomain was catalyzed by ADAM17 (TACE), as demonstrated by selective inhibitor Ro32-7315 and by ADAM17-deficient cells. Shedding and apoptosis were inhibited by the radical scavenger $N$-acetyl-L-cysteine (NAC), indicating that ROS play a pivotal role in both effects. In contrast, pan-caspase inhibitor zVAD-fmk blocked bortezomib-induced apoptosis but not CD30 shedding and TACE-selective metalloproteinase inhibitor Ro32-7315 blocked shedding but allowed apoptosis.

Conclusion: Despite the established clinical value of the proteasome inhibitor bortezomib inducing apoptosis in certain tumor cells, it might also impede the therapy through the removal of target antigens and the release of competitors of the host immune surveillance, utilizing reactive oxygen species in both pathways.

Disclosure: No conflict of interest disclosed.
P851

\section{Insights into the role of ASPP2 (Apoptosis stimulating protein of $\mathrm{p53-2}$ ) in lymphomagenesis}

\author{
Kampa-Schittenhelm, K.M. ${ }^{1}$; Mueller, S. ${ }^{1}$; Bonin, M. ${ }^{2}$; Kanz, L. ${ }^{1}$ \\ Schittenhelm, M.M. ${ }^{1}$; Lopez, C.D. ${ }^{3}$ \\ ${ }^{1}$ Universitätsklinikum Tübingen, Med. II Hämatologie, Onkologie, \\ Rheumatologie, Immunologie, Pulmologie, Tübingen, Germany, \\ ${ }^{2}$ Microarray Facility, Tübingen, Germany, ${ }^{3}$ Oregon Health and Science \\ University, Hematology and Medical Oncology, Portland, United States
}

Introduction: The p53 pathway is a central apoptotic mediator. Its inactivation is common in human tumors resulting in failure of apoptosis promoting oncogenesis. ASPP2 is a p53 binding protein that enhances apoptosis in part through selective stimulation of $\mathrm{p} 53$ transactivation of pro-apoptotic target genes. Low ASPP2 expression is found in many human cancers and has been associated with poor clinical outcome in patients with aggressive lymphoma. Using an ASPP2+/- mouse model, we previously demonstrated that ASPP2 is a haploinsufficient tumor suppressor that cooperates with p53, and that reduced ASPP2 expression results in attenuated damage-response thresholds (Kampa et al., PNAS 2009). However, the underlying mechanisms of tumor suppression remain to be elucidated. While ASPP2-/- mice are not viable, ASPP2+/- mice have an increased incidence of various spontaneous cancers and develop especially high-grade thymic T-cell lymphomas after g-irradiation. Consistently, primary ASPP2+/- thymocytes have an attenuated apoptotic response after g-irradiation compared to ASPP2+/+ thymocytes.

Methods: To explore the mechanism how attenuated ASPP2 expression attenuates apoptosis and increases thymic lymphomagenesis, we performed global gene expression profiling on unirradiated and 5 Gy irradiated ASPP2+/+ and ASPP2+/- thymocytes using an Affymetrix Mouse GeneChip ${ }^{\circledR}$ Array. Differential expression of the identified genes was verified by qRT-PCR and Western Blot.

Results: We found significant differences in gene expression between ASPP2+/+ and ASPP2+/- thymocytes in both unirradiated and irradiated sets. Attenuated activation of p53 target genes like Puma, Bax, Noxa and p21 was detected in ASPP2+/- thymocytes. Moreover differences in numerous p53 independent genes intimately involved in pathways associated with cell growth, tumor formation, hematologic malignancies and immune responses were observed.

Conclusion: We demonstrate that reduced ASPP2 expression affects p53 dependent as well as p53 independent pathways in both transcriptional as well as post-transcriptional networks intimately involved in the development of hematologic disorders. Reduced ASPP2 levels result in global dysregulation of pathways engaged in tumor suppression networks and the cellular damage response, which may ultimately promote genomic instability and tumor formation. Our findings provide insights into the role of ASPP2 in lymphomagenesis and reveal possible new targets for cancer therapy.

Disclosure: No conflict of interest disclosed

\section{P852}

Quality of life of long term survivors with hodgkin's lymphoma after high-dose chemotherapy with autologous stem cell transplantation and conventional chemotherapy

\author{
Brandt, J. ${ }^{1}$; Dietrich, S. ${ }^{1}$; Meissner, J. ${ }^{1}$; Neben, K. ${ }^{1}$; Ho, A. ${ }^{1}$; \\ Witzens-Harig, $M$.
}

${ }^{1}$ Universitätsklinik Heidelberg, Hämatologie und Onkologie, Heidelberg, Germany

Introduction: High-dose chemotherapy (HDCT) followed by peripheral blood stem cell transplantation (PBSCT) is frequently applied in eligible patients with relapsed or refractory Hodgkin's disease. The toxicity of HDCT, however, might manifest itself in the respective patients' reduced quality of life (QoL). In this study we investigated the QoL of long term survivors after HDCT in comparison with patients after conventional chemotherapy and the healthy German population.

Patients and methods: QoL was evaluated with two standardized questionnaires: EORTC QLQ-C30 and EQ-5D. A total of 98 patients were included in the study. 37 patients with a median age of 46 received HDCT with PBSCT between 1986 and 2007. The median follow up of this group was 11 years. 
This group was compared with 61 patients with a median age of 41 treated with conventional chemotherapy and supplementary radiation in our institution. In addition, Qol of the patients was compared to QoL of healthy people on the basis of two studies about the general health status of the German population.

Results: In the HDCT group, the results of the questionnaires show a reduced QoL compared to the healthy population. In the EORTC QLQ-C30 questionnaire, the one sample t-test evaluation shows that the decrease in QoL is significant with $\mathrm{p}<0.05$ in all of the subcategories of the functional state and four of the nine subcategories of the symptomatic state, namely fatigue, dyspnoea, diarrhoea and financial difficulties. Compared to the group of patients who received conventional chemotherapy, there is a tendency towards reduced QoL in patients with HDCT in all of the three main categories of the EORTCQLQ-C30, in particular in the category of symptomatic state. However, these differences were not statistically significant, with the exception of the subcategory of dyspnoea $(\mathrm{p}<0.001)$

Conclusions: In this long term follow up study, QoL is reduced in patients after HDCT as well as in patients after conventional treatment compared to the healthy population. In the HDCT group, more patients are suffering from dyspnoe than in the conventional therapy group, possibly due to BCNU containing high dose regimens. We conclude that the negative impact of both HDCT and conventional therapy on the QoL of long term survivors with Hodgkin's lymphoma should not be underestimated and should lead to the development of less toxic therapy strategies.

Disclosure: No conflict of interest disclosed.

\section{P853}

\section{Ultrasound guided splenic biopsy}

Klühs, L. ${ }^{1}$; Meier, A. ${ }^{2}$; Schuler, M. ${ }^{3}$; Benter, $T^{2}$

${ }^{1}$ HELIOS Klinikum Berlin-Buch, Berlin, Germany, ${ }^{2}$ Asklepios Klinikum Uckermark, Schwedt, Germany, ${ }^{3}$ Medizinische Fakultät Carl Gustav Carus Universität Dresden, Dresden, Germany

Objective: The objective of this study is to evaluate the efficacy and safety of ultrasound-guided percutaneous splenic biopsies as diagnostic procedure. We performed a retrospective review of our experiences with percutaneous splenic interventions and examined both the success rate and the complication rate.

Materials: Between 1997 to December 2009 about 3500 biopsies were performed. Of these, twenty-nine were sonographically-guided percutaneous splenic procedures in 29 patients ( $0,7 \%$ compared to $23,4 \%$ liver biopsies). A $4 \mathrm{MHz}$ ultrasound probe was used with four different ultrasound apparatuses. Biopsies were performed with $18 \mathrm{G}$ true cut needles $(0,95-1,2 / 15-22 \mathrm{~mm})$ using either the subcostal or the intercostal approach. In nine cases fibrin gluten was used to close the branch canal. Twenty-six patients were monitored over 24 hours, whereas three were out-patients. Diagnosis were assured by clinical course or histologies (bone marrow, whole spleen). Indications for biopsy:

1. focal spleen lesions (18 pat.)

2. progress of splenomegaly and B-symptoms (6 pat.)

3. suspicion of diffuse infiltration (3 pat.)

4. spleen abscess (1 pat.)

5. accessory spleen/Gist-filia (1 pat.)

Results: Splenic biopsy was successful in 25 cases of the procedures. We found 9 cases of aggressive B-NHL and one relapse; 5 follicle center lymphomas, and one each: hemangioma, hamartoma, Hodgkin-disease, medulloblastoma, mantle-cell lymphoma, sarcoidosis, abscess (streptococcus constellatus), accessory spleen und AIHA. In 2 cases we could not find the causation and the splenomegaly was regressive. Together, 16 maligne $(61,5 \%)$ and 10 benigne $(38,5 \%)$ cases have been seen. No bleeding or pain after the procedures could be observed.

Histology:

False negative 7 sensitivity t 56,25\%

true negative 10 Spezifität $100 \%$

true positive 9 pos. predictive value $100 \%$

False positive 0 neg. predictive value $58,8 \%$

efficiency $73,1 \%$

Conclusion: This study suggests that splenic biopsy can be performed safely and is of considerable value in diagnosis. No complications were considered after the procedures. In cases of potential bleeding a fibrin gluten can be used.
For indication the therapeutic relevance and diagnostic alternatives were crucial.

Disclosure: No conflict of interest disclosed.

\section{P854}

\section{Schiff base transition metal complexes induce strong antileukemia and antilymphoma activity}

Hille, A. ${ }^{1}$; Wolf, T. ${ }^{2}$; Schumacher, P.2; Ott, I. ${ }^{1}$; Gust, R. ${ }^{1}$; Kircher, $B^{2}$

${ }^{1}$ Freie Universität Berlin, Institut für Pharmazie, Berlin, Germany, ${ }^{2}$ Medizinische Universität Innsbruck, Universitätsklinik für Innere Medizin V - Hämatologie \& Onkologie, Innsbruck, Austria

Schiff base transition metal complexes exert various biological effects like antifungal, antibacterial and even antitumor activities. Therefore, they are used as attractive lead structures for the design of cytostatics with a mode of action that differs from that of cisplatin and its second-generation derivatives, which still induce non-tolerable side-effects and resistance. Data on antileukemic and antilymphoma effects of Schiff base transition metal complexes, however, are limited.

The activity of salophene (N,N'-bis(salicylidene)-1,2-phenylenediamine), its iron(II/III) and manganese(II/III) complexes as well as methoxy-substituted iron(III) salophene complexes was evaluated against the HL-60, SUP-B15 and K-562 leukemia and the U-937 non-Hodgkin's lymphoma cell lines.

The free ligands induced in all cell lines, if at all, only marginal, concentration-dependent antiproliferative effects, and did not trigger $\mathrm{Cu} / \mathrm{Zn}$ superoxide dismutase (SOD) release or induce apoptosis. The Schiff base transition metal complexes $\left[\mathrm{Fe}^{\mathrm{II}}\right.$ (salophene) $]$ and $\left[\mathrm{Fe}^{\mathrm{III}}(\right.$ salophene $\left.) \mathrm{Cl}\right]$ blocked cellular proliferation, caused a strong release of $\mathrm{Cu} / \mathrm{Zn}$ SOD and induced apoptosis. The methoxy-substituted iron(III) complexes $\left[\mathrm{Fe}^{\mathrm{III}}\right.$ (4-OMe-salophene) $\mathrm{Cl}$ and $\left[\mathrm{Fe}^{\mathrm{III}}\right.$ (6-OMe-salophene)Cl were even more active than the regioisomers. In contrast, the manganese analogs $\left[\mathrm{Mn}^{\mathrm{II}}\right.$ (salophene) $]$ and $\left[\mathrm{Mn}^{\mathrm{III}}\right.$ (salophene) OAc] inhibited cell growth, caused the programmed cell death only at higher concentrations and did not provoke release of $\mathrm{Cu} / \mathrm{Zn}$ SOD in any of the four cell lines.

In conclusion, Schiff base transition metal complexes induce strong inhibitory effects on human leukemia and lymphoma cells.

Disclosure: No conflict of interest disclosed.

\section{P855}

\section{Lymphoma-like course of aggressive multisystem Langerhans cell histiocytosis in an adult patient: Case report}

Szturz, P.1; Adam, Z. ${ }^{1}$; Řehák, Z. 2; Koukalová, R. ${ }^{2}$; Nebeský, T.3.; Neubauer, J. ${ }^{3}$; Moulis, M. ${ }^{4}$; Mayer, J. ${ }^{1}$

${ }^{1}$ Faculty of Medicine of Masaryk University and University Hospital Brno, Department of Internal Medicine - Hematooncology, Brno, Czech Republic, ${ }^{2}$ PET Centre at the Masaryk Memorial Cancer Institute, Department of Nuclear Medicine, Brno, Czech Republic, ${ }^{3}$ Faculty of Medicine of Masaryk University and University Hospital Brno, Radiological Clinic, Brno, Czech Republic, ${ }^{4}$ Faculty of Medicine of Masaryk University and University Hospital Brno, Institute of Pathology, Brno, Czech Republic

Langerhans cell histiocytosis ( $\mathrm{LCH})$ is a rare idiopathic disease characterized by clonal proliferation of Langerhans dendritic cells in various tissues and organs. The case report given here describes an unusual case of a 35-year otherwise healthy man diagnosed with aggressive form of LCH initially taking course under the form of lymphoma with expressed B-symptoms (night sweats, fever and weight loss) and generalized peripheral lymphadenopathy. Also present were productive cough and perianal itching. The diagnosis was determined from lymph node and perianal skin biopsies. Furthermore, by a typical HRCT finding, pulmonary involvement had been confirmed the gradual development of which we succeeded to document through several HRCT and PET/CT scans from its initial florid phase characterized by disseminated nodularities up to the terminal phase with the decline of activity and development of cystic formations. After the collection of peripheral blood stem cells, 
the planned patient's therapy started which in all consisted of three monotherapy cycles with cladribine followed by three cycles of combined chemotherapy (cladribine + cyclophosphamide + methylprednisolone) and complemented with curative radiotherapy of the perianal area. This treatment put the disease into complete remission. However, in two months the initial B-symptoms occurred again, along with the pulmonary symptomatology, perianal pruritus and newly also with hip bone pains. The suspected LCH relapse was proved histologically by lymph node biopsy and confirmed on a restaging PET/CT examination which also showed disease dissemination into the hip bones. Thus, an aggressive form of the disease with early relapse had been the case which was indicated for administering 4 cycles of CHOEP as salvage regimen completed in March 2010 with high-dose BEAM chemotherapy followed by autologous peripheral blood stem cell transplantation. Therefore, generalized involvement of nodes doesn't always need to be a malignant lymphoma or metastatic dissemination of a tumour but also LCH may be the case. The presence of B-symptoms may very likely stand for an aggressive form of the disease. Proving beneficial in classic forms of $\mathrm{LCH}$, therapy with cladribine (2-chlorodeoxyadenosine) in cases of highly aggressive forms of LCH doesn't need to have the same effect as in $\mathrm{LCH}$ with low proliferative activity, which conforms to the present experience in the treatment of indolent and aggressive lymphomas.

Disclosure: No conflict of interest disclosed.

\section{Posterdiskussion Stammzellbiologie \\ P856 \\ Adipogenic differentiation of human mesenchymal stromal cells is up-regulated by microRNA-371 and down-regulated by microRNA-369-5p}

\section{Bork, S. ${ }^{1,2}$; Horn, P. ${ }^{1}$; Castoldi, M. ${ }^{3}$; Ho, A.D..$^{1}$; Wagner, W. ${ }^{1,4}$}

${ }^{1}$ Department of Medicine V, University Hospital of Heidelberg, Heidelberg, Germany, ${ }^{2}$ Heidelberg Academy of Sciences and Humanities, Heidelberg, Germany, ${ }^{3}$ Department of Pediatric Oncology, Hematology and Immunology, University Hospital of Heidelberg, Heidelberg, Germany, ${ }^{4}$ Helmholtz-Institute for Biomedical Engineering, Aachen University Medical School, Aachen, Germany

Introduction: Long-term culture of human mesenchymal stromal cells (MSC) has implications on their proliferation and differentiation potential. Recently, we have demonstrated that this is also associated with up-regulation of the five microRNAs (miRNAs) miR-29c, miR-369-5p, miR-371, miR-499 and let-7f. In this study we have now addressed the role of these senescence-associated miRNAs for cellular aging and differentiation of MSC.

Methods: To determine the functional role of these miRNAs we transfected young MSC with the different miRNAs or anti-miRNA molecules and examined the effect on the adipogenic and osteogenic differentiation potential and replicative senescence of MSC.

Results: We could demonstrate that adipogenic differentiation was significantly impaired upon transfection with mir-369-5p whereas it was highly increased by mir-371. These differences were also detected on gene expression level. Transfection with miR-369-5p impaired up-regulation of the adipogenic associated markers adiponectin (ADIPOQ) and fatty acid binding protein 4 (FABP4) whereas transfection with an inhibitor of miR-369-5p increased expression of ADIPOQ even under non-differentiation conditions. miR-371 stimulated expression of ADIPOQ and FABP4 under non-differentiation conditions and this is consistent with the observed positive effect on adipogenic differentiation. Microarray analysis upon adipogenic or osteogenic differentiation revealed down-regulation of several miRNAs albeit miR-369-5p and miR-371 were not affected. Therefore is conceivable that these two miRNAs are up-stream regulators rather than effectors of adipogenic differentiation. Additionally, it has been suggested that DNA methylation plays a role in replicative senescence and in vitro differentiation. Therefore we analyzed the effects of the five miRNAs on the expression of de novo DNA methyltransferases. Transfection of miR-29c decreased expression of DNMT3A and DNMT3B. In addition, miR-369-5p reduced gene expression of DNMT3A, whereas miR-371 stimulated significantly expression of DNMT3A and DNMT3B.
Conclusions: These data support the notion that miR-369-5p and miR-371 are important regulators of adipogenic differentiation in MSC and this might be mediated by epigenetic modifications.

Disclosure: No conflict of interest disclosed.

\section{P857}

\section{Age-dependent methylation pattern in hematopoietic} progenitor cells

Hellwig, I. ${ }^{1}$; Bocker, M. ${ }^{2}$; Reu, F.1.; Taubert, I. ; Eckstein, V.1; Lyko, F. ${ }^{2}$; Ho, A.D.

${ }^{1}$ Universitätsklinikum Heidelberg, Innere Medizin V, Stammzelllabor AG Ho, Heidelberg, Germany, ${ }^{2}$ Deutsches Krebsforschungszentrum, Epigenetics, Heidelberg, Germany

Introduction: DNA methylation of CpG-Islands plays an important role in the regulation of gene expression. It is known as an key regulator in development as well as in maintenance and differentiation of stem cells. Aberrant DNA methylation was found to play a role in formation of several human diseases including cancer. We hypothesized that, changes in DNA methylation contributes to reduced self-renewal of hematopoietic progenitor cells (HPC).

Methods: We studied the genome wide methylation pattern in HPC isolated from 5 umbilical cord bloods (cb) and 5 healthy voluntary donors of mobilized peripheral blood (pb), at the age of 28 to 40 years. Therefore, we applied the Illumina Infinium human-methylation 27 BeadChip which examines 27'580 CpG-Islands accordingly to more than $14^{\prime} 000$ genes. In this study allosomes were excluded from analysis because the gender of cb-Blood was unknown. As control we used the differentiated blood cells Granulocytes and Monocytes. Methylation data will be validated by 454 deep bisulfite sequencing.

Results: There are various different methylated $\mathrm{CpG}$ in between the two groups of HPCs, isolated from infants (cb) versus older donors (pb), even if the overall Pearson correlation coefficient is 0,979 . The narrow methylation difference is set at $20 \%$ more or less methylation in pb-cells minus cb-cells. Almost twice CpG-Islands are more methylated in older HPCs ( $\mathrm{pb}$ ) compared to young ones (cb). 202 CpG-Islands, respectively 194 genes, showed a decreased methylation in aged stem cells. A gain of methylation was measured at $136 \mathrm{CpG}$-sites, according to 128 genes. The methylation pattern within the cb-cells, as well as in the pb-cells, is unexpected homogeneous. The promoter DNA of stemmness factors as e.g. CD34, KLF 4 and Sox 2 are almost unmethylated in young as well as in aged HPC (R-value 0,99$)$.

Conclusion: HPCs from the same age show a very similar pattern which implicates a change of DNA methylation pattern during the aging of stem cells. Furthermore it is crucial to point out the importance of DNA methylation for gene expression by semi quantitative real-time RT-PCR. In further experiments it is important to investigate functional differences between the two groups by pathway analysis and functional assays. Therewith it could be possible to identify epigenetic changes, relative to the age of the donor, that confer a risk for development of bone marrow disease warranting therapeutic intervention.

Disclosure: No conflict of interest disclosed.

\section{P858}

DNA methylation changes upon aging and long-term culture of mesenchymal stromal cells and dermal fibroblasts

Koch, C. ${ }^{1}$; Suschek, C. ${ }^{2}$; Bork, S. ${ }^{3}$; Pfister, S. ; Witt, H. ${ }^{4}$; Pallua, N. ${ }^{2}$; Arden, N. ${ }^{1}$; Ho, A.D. ${ }^{3}$; Zenke, M. ${ }^{1}$; Wagner, W. ${ }^{1}$ ${ }^{1}$ Helmholtz Institute for Biomedical Engineering, Department for Cell Biology, University of Aachen, Aachen, Germany, ${ }^{2}$ Department for Plastic Surgery, University of Aachen, Aachen, Germany, ${ }^{3}$ Department of Medicine V, University of Heidelberg, Heidelberg, Germany, ${ }^{4}$ Molecular Genetics of Pediatric Brain Tumors, German Cancer Research Center (DKFZ), Heidelberg, Germany

Introduction: We have recently demonstrated significant changes in the global DNA-methylation pattern of mesenchymal stromal cells (MSC) upon long-term culture and aging. Similar epigenetic modifications might also 
occur upon aging of related cell types such as fibroblasts. To address this question, we have now analyzed DNA-methylation profiles in dermal fibroblasts from different body regions and from young and elderly donors.

Methods: DNA methylation profiles were analyzed by using the novel HumanMethylation27 BeadChip platform. This technique allows simultaneous analysis of 27,578 unique $\mathrm{CpG}$ sites. Age-associated methylation changes were exemplarily validated for CDKN2B by pyrosequencing.

Results: Fibroblasts revealed the same morphology and immunophenotype as MSC, however, adipogenic and ostogenic differentiation could only be induced in MSC but not in fibroblasts. DNA-methylation profiling revealed significant differences between fibroblasts and MSC. Interestingly, hierachica clustering demonstrated a close relation between samples from the same dermal region and this supports the notion of differences between fibroblasts from distinct body parts. Overall the methylation pattern was similar in fibroblasts from young and elderly donors but some specific $\mathrm{CpG}$ sites revealed significant age-associated changes. Notably, CDKN2B was hyper-methylated upon aging in fibroblasts, whereas it was hypo-methylated in MSC. Long-term culture induced similar epigenetic modifications in fibroblasts and MSC.

Conclusion: These results point to an epigenetic regional memory of fibroblast and specific $\mathrm{CpG}$ sites become differentially methylated upon aging. Methylation changes upon long-term culture correlated in fibroblasts and MSC, indicating that cellular aging is governed by similar epigenetic modifications.

Disclosure: No conflict of interest disclosed.

\section{P859}

In vitro analysis of intracellular signalling events by flow cytometry using phospho-specific antibodies in haematopoietic progenitor cells

Munjal, U. ${ }^{1}$; Boehme, S. ${ }^{2}$; Kollar, K. ${ }^{2}$; Heinz-Senf, P. ${ }^{2}$; Deak, E. ${ }^{2}$; Seifried, E. ${ }^{2}$; Twja, M. ${ }^{2}$; Henschler, R. ${ }^{2}$

${ }^{1}$ III. Medizinische Klinik, Universitätsmedizin Mannheim, Mannheim, Germany, ${ }^{2}$ DRK Blutspendedienst Baden-Württemberg-Hessen, Frankfurt, Germany

Background: Recent advances in intracellular staining techniques, fluorescent reagents, and antibody production have advanced the number of intracellular antigens that can be analyzed by flow cytometry. Measurement of protein phosphorylation with phospho-specific antibodies has provided insight into kinase signaling cascades. However, in cellular therapy, the activation status of intracellular signal transduction proteins such as tyrosine, serine and theronine kinases have not yet been elucidated. In this study, we aimed to analyze the activation status of two intracellular signalling pathways in hematopoietic progenitor cell populations.

Materials and methods: In vitro expanded FDCP-mix cells (a multipotent cell line of hematopoietic stem cells which have the ability to differentiate along the myeloid and erythroid lineages) and human bone marrow-derived mesenchymal stromal cells (MSC) at passage 5-9 were stimulated with phorbol ester. Cells were fixed in $1.5 \%$ PFA, washed in methanol buffer and incubated with Alexa flour 647 conjugated anti P-STAT3 P-Ser 727and antiERK1/2 P-Tyr Y204 antibody in different concentrations $(0-20 \mu 1)$. The cells were washed and resuspended in staining media and analysed by flow cytometry using the "multiplex staining by fluorescent- cell barcoding" (SFB project 834: Endothelial signalling and Vascular repair).

Results: We found that FDCP-mix cells showed a dose-dependent activation of ERK1/2 and STAT3 whereas stimulation of MSC with phorbol ester resulted in a strong induction of pSTAT3 (10 $\mu$ or above). Stimulation of both FDCP mix cells and MSC resulted in a strong induction of pSTAT3 and pERK $1 / 2$ at $10 \mu \mathrm{l}$ or above. Depletion of IL3 (growth factor) in cell cultures did not improve the phosphorylation efficiency of FDCP mix cells or MSC. These findings indicate that FDCP-mix cells and MSC show little constitutive activity of ERK1/2 and STAT3 phosphorylation. Furthermore, when cells were incubated with a JAK inhibitor (benzimidazol-isoquinolone derivative) reduced induction of STAT3 was shown, confirming specificity of the detected signal. Conclusions: We have shown the phosphorylation of ERK1/2 and STAT3 by using the phospho-flow technique. The use of phospho-specific antibodies provides an alternative to classical analysis methods allowing us to examine intracellular signalling pathways which have previously not been possible.

Disclosure: No conflict of interest disclosed.
P860

Analysis of SDF-1 $\alpha$ expression in hMSC for the experimental investigation of hematopoietic stem cell chemotaxis

Leinweber, C. ${ }^{1}$; Saffrich, R. ${ }^{2}$; Ho, A.D. ${ }^{2}$; Grunze, M. ${ }^{1,3}$; Rosenhahn, A. ${ }^{1,3}$

${ }^{1}$ Angewandte Physikalische Chemie, Universität Heidelberg, Heidelberg, Germany, ${ }^{2}$ Innere Medizin V, Universitätsklinikum Heidelberg, Heidelberg, Germany, ${ }^{3}$ Institut für Funktionelle Grenzflächen (IFG), Karlsruhe Institute of Technology (KIT), Karlsruhe, Germany

Introduction: Human mesenchymal stromal cells (hMSC) can be used as a niche model for the experimental investigation of the chemotactic migration of human hematopoietic stem and progenitor cells (HSC) towards their stem cell niche, the so called homing process. These bone marrow derived hMSC express the messenger chemokine stromal cell-derived factor-1 alpha (SDF-1 $\alpha$ ). Because the exact sensing mechanism and migration behavior of HSC is still not completely unraveled, we studied migration of human HSC under laboratory conditions using several chemotaxis experiments with increasing intricacy from single well up to microfluidic devices.

Methods: For the definition of chemical gradients inside these setups we explored in detail the expression of the chemokine SDF- $1 \alpha$ in hMSC applying ELISA tests (RayBiotech). The expression behavior has been studied dependent on culture time, cell amount and culture media. Additionally we performed intracellular immunofluorescence staining of SDF- $1 \alpha$ in hMSC to support the ELISA results. Human HSC (CD34+) for migration experiments were derived from cord blood or mobilized peripheral blood. Both, HSC and $\mathrm{hMSC}$, as well as recombinant SDF- $1 \alpha$ were used to investigate HSC chemotaxis in microwell, transwell and microfluidic migration setups.

Results: The media test clearly shows that SDF- $1 \alpha$ expression in $\mathrm{hMSC}$ is strongly dependent on culture conditions. Highest expression rates and values were obtained within a commercial MSC culture medium (Lonza). Therein SDF-1 $\alpha$ expression increases with increasing cell amount and longer culture time. About 24 hours after a medium exchange an equilibrium plateau concentration in the medium supernatant (about $200 \mathrm{pg} / \mathrm{ml}$ for 50.000 cells) is reached in most cases. The chemotactic response of HSC correlates with the expected development of chemokine gradients according to the measured production rates and diffusion profiles inside the applied microwell and microfluidic setups.

Conclusions: With our study we demonstrate the importance of hMSC culture conditions in planning in-vitro chemotaxis and homing experiments with hMSC as a niche model. SDF- $1 \alpha$ expression data measured with ELISA can be used to calculate chemical gradients inside of microfluidic setups. Thus it is possible to determine sensing thresholds and sensitivity of HSC in response to SDF-1 $\alpha$. Furthermore we showed the applicability of a simple microfluidic setup in experimental medical research.

Disclosure: No conflict of interest disclosed.

\section{P861}

\section{Soluble CD40L is released by matrix metalloprotease-2} (MMP-2)-dependent shedding of platelets and accumulates in stem cell products of autologous donors

Wenzel, F. ${ }^{1}$; Rox, J. ${ }^{2}$; Reinboldt, S. ${ }^{3}$; Weber, A.A. ${ }^{3}$; Giers, G. ${ }^{4}$; Fischer, J. ${ }^{1}$

${ }^{1}$ Universitätsklinik Düsseldorf, Institut für Transplantationsdiagnostik und Zelltherapeutika, Düsseldorf, Germany, ${ }^{2}$ Universitätsklinik Düsseldorf, Düsseldorf, Germany, ${ }^{3}$ Universitätsklinik Essen, Pharmakologie, Essen, Germany, ${ }^{4}$ Universitätsklinik Düsseldorf, Institut für Hämostaseologie und Transfusiosnmedizin, Düsseldorf, Germany

Introduction: Conditional on apheresis techniques, stem cell products contain a considerable amount of thrombocytes. Platelets are the major source of soluble CD40 ligand (sCD40L) (1) in the blood. It has been demonstrated that CD40L is cleaved from the surface of activated platelets. sCD40L is well known to show immun-modulatory functions and high concentrations in blood products (2). Therefore we examined sCD40L concentrations in stem cell apheresis. 
Material and Methods: In five patients suffering from multiple myeloma and undergoing autologous stem cell apheresis, sCD40L concentrations were measured in peripheral blood samples before, during and after apheresis procedure and in the respective stem cell product. sCD40L concentrations were determined by a commercially available ELISA Kit (R\&D Systems). In an additional approach, platelet-rich plasma (PRP) from healthy volunteers $(n=5)$ was incubated with different pharmacological inhibitors (MMP-2/MMP-9 Inhibitor I, MMP-9 Inhibitor I, MMP-2 Inhibitor I, recombinant ADAM 10, and recombinant ADAM-17) during platelet activation.

Results: During stem cell apheresis, a decrease in platelet count could be observed from $95,070 / \mu \mathrm{L} \pm 58,234 / \mu \mathrm{L}$ at the beginning to $54,638 / \mu \mathrm{L} \pm 26,944 /$ $\mu \mathrm{L}$ at the end of the procedure. The thrombocyte loss was accompanied by a significant lowering of sCD40L concentrations in peripheral blood samples from $239 \mathrm{pg} / \mathrm{mL} \pm 139 \mathrm{pg} / \mathrm{mL}$ to $126 \mathrm{pg} / \mathrm{mL} \pm 71 \mathrm{pg} / \mathrm{mL}$ (dependent on platelet count, linearly correlated, $r=0.95$ ). In stem cell products, $\mathrm{SCD} 40 \mathrm{~L}$ concentrations were manifold elevated (range from 2239 to $3641 \mathrm{pg} / \mathrm{mL}$ ) in comparison to concentrations of peripheral blood samples. Using the MMP-9 inhibitor $(100 \mathrm{nM})$ and the MMP-2/9 inhibitor $(3 \mu \mathrm{M}) \mathrm{sCD} 40 \mathrm{~L}$ release by platelets could be inhibited by $>60 \%$. Interestingly, the MMP-2 inhibitor (17 $\mu \mathrm{M})$ completely prevented the shedding of sCD40L from activated platelets. Conclusions: During stem cell apheresis, sCD40L concentrations in periph eral blood were mainly influenced by alterations of platelet count. As known from platelet concentrates, an accumulation of SCD40L could also be observed in stem cell products pointing out the importance of $\mathrm{SCD} 40 \mathrm{~L}$ release by platelets. Additionally, these data support the hypothesis that MMP-2 might be the protease, primarily responsible for sCD40L cleavage from platelet surface. References:

1. Wenzel et al.: Clin Hemorheol Microcirc 2008; 39: 417-22

2. Khan et al.: Blood. 2006; 108: 2455-62

Disclosure: No conflict of interest disclosed.

\section{P862}

In vitro oxygen tension - a critical player in Hematopoietic stem cell co-cultures with mesenchymal stromal cells

\section{Jing, D. ${ }^{1}$; Poitz, D. ${ }^{2}$; Wobus, M. ${ }^{1}$; Bornhäuser, M. ${ }^{1}$; Ehninger, G. ${ }^{1}$;} Ordemann, R. $^{1}$

${ }^{1}$ Medizinische Klinik 1, Stammzelllabor2, Technische Universität Dresden, Dresden, Germany, ${ }^{2}$ Labor für experimentelle und molekulare Kardiologie, Technische Universität Dresden, Dresden, Germany

Background: Hematopoietic stem cells (HSCs) are regulated by stem cell niches in vivo. To mimic the in vivo condition, co-culture assays with mesenchymal stromal cells (MSCs) were performed in vitro. Recently we could demonstrate three spatial compartments as distinct HSC niches in the co-culture. In this study we investigated the impact of oxygen tension on HSC distribution and behavior in the three compartments.

Design and methods: Oxygen tension in the co-culture was evaluated by pimonidazole staining. "HSC distribution ratios" were studied according to cell counts in the three compartments. Hypoxia co-culture were compared with nomoxia co-culture for the characteristics of HSC subsets (i.e. CD34 expression, cell division, migratory capacity) and cytokine secretion by MSCs (i.e. SDF-1 and VEGF-A)

Results: In normoxia co-culture the most hypoxic area was located at the bottom beneath the MSC layer where the reservoir for more primitive HSCs in vitro is located shown recently. In hypoxia co-culture, cell adhesion on MSC layer was diminished (proportion of adherent HSCs at day five: $51 \%$ in nomoxia v.s. 29\% in hypoxia), whereas cell migration beneath MSC layer was increased (proportion of migrated HSCs at day five: $7 \%$ in nomoxia v.s. $22 \%$ in hypoxia). Interestingly SDF-1 secretion by MSCs was down regulated by hypoxia ( $352 \mathrm{pg} / \mathrm{ml}$ in normoxia co-culture v.s. $182 \mathrm{pg} / \mathrm{ml}$ hypoxia co-culture) The capacity of HSCs division was decreased as well. The phenotype of HSCs was maintained in all three compartments of hypoxia co-culture over time in comparison to the normoxia culture. However the distribution of more primitive HSCs was still depending on the spatial localization. In addition we could demonstrate that silencing VEGF-A could enhance cell adhesion on MSC surface in hypoxia co-culture.

Conclusions: Similar to in vivo HSC niche, the in vitro niche in co-culture may also require hypoxia for HSC maintenance. In our experiments hypoxia caused modification of HSCs phenotype, migration and division capacity.
Therefore our data suggest that oxygen tension is critical for stem cell distribution into the proper niche for maintenance in vitro.

Disclosure: No conflict of interest disclosed

\section{P863}

\section{Effects of high dose radiation on hMSC: Proliferation, clonogenicity, differentiation and phenotype}

\section{Schmidtke-Schrezenmeier, G. ${ }^{1}$; Fekete, N. ${ }^{2}$; Rojewski, M. ${ }^{1}$;} Schrezenmeier, $H_{.}{ }^{1,2}$, CASCADE - 7th EU Framework Programme ${ }^{1}$ Universität Ulm, Institut für klinische Transfusionsmedizin, UIm, Germany, ${ }^{2}$ Rotes Kreuz Blutspendedienst Baden-Württemberg - Hessen, Institut für Klinische Transfusionsmedizin und Immunogenetik, Ulm, Germany

Background: Cell therapy with mesenchymal stem/stromal cells (MSCs) is of high interest in varying indications nurtured by their high proliferative capacities. The flip side is the potential risk of tumorigenicity of these cells. However, there is increasing evidence that at least some of the effects of MSCs are exerted through release of cytokines. Long term persistence or MSC may not always be necessary. One method of minimizing this risk could be irradiation of MSCs. Irradiation (usual standard with $30 \mathrm{~Gy}$ ) is a routine procedure in transfusion medicine to prevent long term persistence of nucleated cells. Methods: Different MSC lines were irradiated with either 30 or $60 \mathrm{~Gy}$. Influence on proliferation was evaluated via CYQUANT® test, cell count after trypan blue staining and photodocumentation on days 7, 14, 21 and 28 after irradiation. Influence on clonogenicity was evaluated via CFU-F assay up to 28 days after radiation. Trilineage differentiation potential was tested via standard protocols and phenotype was determined via Flow Cytometry.

Results: In CYQUANT® proliferation test, MSCs showed a mean drop in proliferation down to $17,9 \%$ (SD $0,9 \%$ ) at day 7 after 30 Gy radiation which recovered to $30,7 \%$ (SD 9,4\%) after 28 days compared to non-radiated cells. $60 \mathrm{~Gy}$ radiation showed a drop of proliferation down to $14,6 \%$ (SD 1,8\%) and recovery up to $23,7 \%$ (SD 6,1\%) after 28 days. Consistent results were obtained in cell count after trypan blue staining. In CFU-F assays non-radiated cells showed reproducible, constant colony formation. In all 4 MSC lines evaluated, single colonies could be detected $14 \mathrm{~d}$ but also at later time points after irradiation. 2 MSC lines even showed colonies after 60 Gy irradiation. Trilineage differentiation (ostoeblasts, chondrocytes, adipocytes) could be shown 4 weeks after irradiation. Distinct changes of the phenotype were observed 8 weeks after irradiation (expression of CD3, CD34, CD45, CD49e, HLA class II, reduced expression of CD105 and HLA class I).

Conclusions: It could be shown that MSC show a irradiation resistance remarkable enough to warrant further studies. After 30 and $60 \mathrm{~Gy}$ irradiation, respectively, a drop in proliferation can be detected; however there are surviving cells able to proliferate and to form colonies. Surviving cells still demonstrated trilineage differentation. Phenotype of MSC 8 weeks after irradiation showed distinct changes. (Supported by EU $7^{\text {th }}$ FP CASCADE; N²23236)

Disclosure: No conflict of interest disclosed.

\section{P864}

\section{Regulatory networks of hematopoietic stem cells and their micro-environment}

Vilne, B. ${ }^{1,2}$; Kröger, M. ${ }^{1}$; Peschel, C. ${ }^{1}$; Mewes, H.W. ${ }^{2}$; Oostendorp, R.A.J. ${ }^{1}$

${ }^{1} \mathrm{Klinikum}$ rechts der Isar der TU München, III. Medizinische Klinik, München, Germany, ${ }^{2}$ Technische Universität München, Department of Genome Oriented Bioinformatics, Freising, Germany

In adult bone marrow, HSC reside within a supportive micro-environmenta niche. Current available data support the idea that this niche integrates both cell-intrinsic and cell-extrinsic signals affecting HSC bahavior. Precise signaling mechanisms coordinating HSC-fate decisions and self-renewal have remained largely elusive. To understand these signals, we co-cultured lineagenegative Sca1+ Kit+ (LSK) and HSC-supporting UG26-1B6 stromal cells, and analysed gene expression changes of both cell types at three different timepoints of co-culture. 
These experiments identified 222 transcripts (157 in hematopoietic and 66 in stromal cells) with expression changes during the time-course experiment. In hematopoietic cells, 79 transcripts were significantly up-regulated ("activated") during co-culture, in comparison to input LSK cells. Preliminary analyses of activation demonstrate changes in metabolism, cell adhesion, transcription, and signal transduction as well as hematopoietic differentiation. Transcripts that were downregulated ("quiescence" markers) included regulators of chromatin structure and transcription as well as transcripts coding for cell cycle proteins. Interestingly, 66 genes were differentially expressed in stromal cells in response to coculture with LSK cells. These included regulators of transcription and the epigenome, as well as growth factors and signal transducers.

We will present an in-depth analysis of these gene expression data during coculture of both hematopoietic and niche cells using integrated bioinformatics aproaches. Our future work is aimed at modelling and experimentally testing dynamic gene regulatory networks. Our studies should generate novel insights into the behavior of HSCs in their micro-environment.

Disclosure: No conflict of interest disclosed.

\section{P865}

\section{Expansion of cord blood CD34+ progenitor cells with combination of cytokines and mesenchymal stromal cells}

Walenda, T. ${ }^{1}$; Bork, S. ${ }^{2}$; Horn, P. ${ }^{2}$; Bokermann, G. ${ }^{1}$; Wein, F.3. Diehmann, A. ${ }^{2}$; Joussen, S. ${ }^{1}$; Drescher, W. ${ }^{4}$; Piroth, D. ${ }^{5}$ Ho, A.D. ${ }^{2}$ Wagner, $W{ }^{1}$

${ }^{1}$ RWTH Aachen Medical School, Stem Cell Biology and Cellular Engineering, Aachen, Germany, ${ }^{2}$ University of Heidelberg, Department for Hematology and Oncology, Heidelberg, Germany, ${ }^{3}$ Swiss Federal Laboratories for Material Testing and Research, St. Gallen, Switzerland, ${ }^{4}$ RWTH Aachen Medical School, Department for Orthopedics, Aachen, Germany, ${ }^{5}$ RWTH Aachen Medical School, Department for Gynecology, Aachen, Germany

Introduction: Umbilical cord blood has become a viable option for transplantation of hematopoietic stem and progenitor cells (HPC). Due to the limited number of stem cells per cord blood unit, in vitro expansion prior to transplantation might provide a strategy to reduce time for hematopoietic recovery after transplantation. Mesenchymal stromal cells (MSC) have been suggested to provide a suitable cellular environment for in vitro expansion of HPC. Here, we have analyzed the influence of MSC co-culture on HPC expansion.

Methods: CD34 ${ }^{+}$cells were isolated from cord blood and cultured with different cytokine combinations either with or without MSC. Division history and differentiation of HPC was monitored using carboxyfluorescein diacetate $\mathrm{N}$-succinimidyl ester (CFSE) and flow cytometric analysis of surface markers.

Results: MSC significantly enhanced the proliferation rate of HPC, especially in the more primitive $\mathrm{CD} 34^{+} / \mathrm{CD} 38^{-}$fraction. Co-culture maintained expression of the stem cell markers CD34 and CD133 for more cell divisions. Up-regulation of differentiation markers CD45 (common lymphocyte antigen), CD13 (myeloid marker) and CD56 (NCAM, expressed on NK-cells) was delayed to a higher number of cell divisions. Furthermore, co-culture facilitated a significant expansion of HPC with primitive immunophenotype, and the highest number of $\mathrm{CD} 34^{+}$cells was achieved in co-culture with MSC in StemSpan expansion medium supplemented with SCF, TPO and FGF Additional supplementation of Angpt15 and IGFBP2 promoted expansion of HPC only under culture conditions without stromal support. Expansion of colony forming unit cells was rather dependent on addition of SCF, TPO and FGF than on stromal support.

Conclusion: MSC support proliferation as well as self-renewal of HPC with primitive immunophenotype. Our data indicate that combination of specific cytokines with stromal cells support further expansion of HPC from umbilical cord blood.

Disclosure: No conflict of interest disclosed.

\section{P866}

Inhibiting effect of lenalidomide on both leukemic CD34+ cells and normal CD34+ progenitors

\author{
Möhle, R. ${ }^{1}$; Mazier, A. ${ }^{1}$; Drost, A. ${ }^{1}$; Kanz, L. ${ }^{1}$; Weisel, K. ${ }^{1}$
}

${ }^{1}$ Med. Klinik II, Univ. Tübingen, Tübingen, Germany

Introduction: The immunmodulatory drug lenalidomide has emerged as a mainstay in multiple myeloma therapy, and in $\operatorname{del}(5 q) \mathrm{MDS}$ in some countries. However, hematotoxicity and suppression of stem cell mobilization are observed during lenalidomide treatment. It is unknown to which extent direct effects on hematopoietic stem and progenitor cells (HPC) or indirect modulation of the hematopoietic microenvironment contribute to these side effects. Methods: To assess direct effects of lenalidomide on CD34+ cells, apoptosis was measured by annexin staining in the CD34+ leukemic progenitor cell line $\mathrm{KG}-1 \mathrm{a}$. In addition, primary $\mathrm{CD} 34+\mathrm{HPC}$ were isolated from G-CSFmobilized peripheral blood and maintained in serum-free liquid culture supplemented with cytokines (SCT, FLT-3, IL-6) and various concentrations of lenalidomide ( $1 \mathrm{nM}$ to $100 \mu \mathrm{M})$. Total cell number, surface antigens, and colony-forming units were measured after 7 days using flow cytometry and methylcellulose colony assays.

Results: In the CD34+ KG1a cell line which shares some features with normal CD34+ cells, $4 \mathrm{~h}$ after exposure to lenalidomide, an increased percentage of apoptotic cells was observed throughout the evaluated dose range from $1 \mathrm{nM}$ to $100 \mu \mathrm{M}$ (maximum at $10 \mu \mathrm{M}, 3.6$-fold compared to control). This effect could be due to the leukemic nature of KG1a including presence of del(5). However, also in cytokine-supplemented liquid culture, the proportion of nonmalignant CD34+ HPC was significantly reduced after 7 days by the presence of lenalidomide in a dose-dependent manner beginning at $100 \mathrm{nM}$ up to $50 \%$ at $100 \mu \mathrm{M}$ lenalidomide. Conversely, the proportion of differentiated CD34- CD45+ myeloid cells increased, whereas only minor changes were observed in the total cell number and in the number of erythroid and myeloid colony-forming units. However, when $10 \mu \mathrm{M}$ lenalidomide was added directly to the semisolid colony assay, mainly erythroid, but also myeloid colony growth of freshly isolated CD34+ HPC was markedly reduced.

Conclusions: These results suggest that lenalidomide may impair growth no only of leukemic, but also of normal CD34+ cells. Direct effects on HPC may be partially responsible for the hematologic side effects of lenalidomide treatment.

Disclosure: Robert Möhle: No conflict of interest disclosed.

Katja Weisel: Advisory Role: Celgene $\mathrm{GmbH}$; Honoraria: Celgene $\mathrm{GmbH}$; Financing of Scientific Research: Celgene $\mathrm{GmbH}$

\section{P867}

$\alpha 6$ and $\alpha 4$ integrin levels on mobilized peripheral blood stem cells predict engraftment velocity in patients receiving autologous stem cell transplantation

Hartz, B. ${ }^{1}$; Loechelt, C. ; Volkmann, T. ${ }^{1}$; Neubauer, A. ${ }^{1}$; Brendel, C. ${ }^{1}$

${ }^{1}$ University Hospital, Philipps University Marburg, Dept. of Hematology, Oncology, Immunology, Marburg, Germany

Latency of leukocyte engraftment in patients receiving peripheral blood stem cell transplantation (PBSCT) is clinically important, because the risk of opportunistic infections increases with prolonged aplastic post transplant phases. Expression of adhesion and chemokine recepters on hematopoietic stem cells (HSC) have been shown to modulate homing and engraftment in murine model systems. Therefore we were interested to correlate engraftment and leukocyte regeneration in patients receiving chemotherapy and G-CSF mobilization for PBSCT with the expression levels of $\alpha 4, \alpha 6$ integrin and CXCR 4 on the CD34+ hematopoietic stem cell compartment . Leukapheres products were stained with fluorochrome labeled antibodies and analysed by multiparameter flow cytometric analysis. Elevated expression levels of $\alpha 6$ integrin in CD34+ cells were highly predictive for rapid stem cell engraftment (day 9-13), while low surface expression was associated with delayed recovery of leukocytes (21-41 days) and thrombocytes (21-61) ( $<<0.0001)$. High expression of the $\alpha 4$ integrin adhesion receptor and the CXCR 4 chemokine receptor were also associated with rapid engraftment after PBSCT. These findings are in line 
with recent data indicating that lack of adhesion receptor expression on HSC restricts self renewal capacity.

Thus, determination of CXR4, $\alpha 4$ and $\alpha 6$ integrin expression on HSC is a useful tool in order to predict the engraftment capacity of mobilized peripheral blood stem cells. In addition drugs that are known to downregulate these adhesion receptors should be handled with care in these patients, while drug induced up-regulation of adhesion molecules on transplanted HSC could be a promising strategy to accelerate engraftment in patients receiving PBSCT.

Disclosure: No conflict of interest disclosed.

\section{P868}

\section{GMP-compliant manufacturing of multipotent stroma cells} including functional potency tests for product release

Nerger, K. ${ }^{1}$; Weber, T. ${ }^{1}$; Lützkendorf, J. ${ }^{1}$; Mögel, A. ${ }^{2}$; Müller, T.1. Tetzlaff, A. ${ }^{2}$; Hering, J.2; Schmoll, H.-J. ${ }^{1}$; Müller, L.P. ${ }^{1}$

${ }^{1}$ Universitätsklinik für Innere Medizin IV, Universitätsklinikum Halle, MartinLuther-Universität Halle-Wittenberg, Halle, Germany, ${ }^{2}$ Einrichtung für Transfusionsmedizin, Universitätsklinikum Halle, Martin-Luther-Universität Halle-Wittenberg, Halle, Germany

Background: Multipotent stroma cells (MSC) provide a tool for new therapeutic approaches in autoimmune diseases and tissue repair as well as for the generation of transgenic cell products. Clinical grade manufacturing has been reported and MSC are used in clinical trials. However, evaluation in clinica studies as well as in prospective routine clinical use requires the standardized production of MSC including release criteria predicting the clinical efficacy and safety of the produced specific batch.

Methods: A SOP-system for the animal-protein-free manufacturing of native and cryopreserved MSC was established and authorized by the federal regulatory agencies. Based on that protocol, complete validation runs were performed in different patients. Apart from microbiological testing and phenotypical analysis several criteria for in-process testing and finished product assessment were defined and evaluated. Additionally, non-GMP MSC were used to develop quality testing and analyze its relevance.

Results: In all patients the protocol allowed isolation and expansion of MSC to a final cell number of $>160 \times 10^{6}$ over a period of $<30$ days and $<40$ population doublings. At the time of release - i. e. at the time of simulated transplantation - cell viability was $>80 \%$ in native and cryopreserved products. However, cell loss due to adherence to the plastic bags was observed Manufactured MSC showed typical phenotype and osteogenic, adipogenic and chondrogenic differentiation potential before and after cryopreservation. In mixed cultures, native and cryopreserved MSC did not elicit a proliferation of native HLA-incompatible leukocytes but suppressed their mitogen-induced proliferation to a limited extent. Apparently this degree of suppression was dependent on the nature of the leukocytes but independent of the degree of HLA-compatibility. Neither native nor cryopreserved manufactured MSC showed malignant transformation in vitro and in vivo.

Discussion: Based on these data a protocol with inclusion of potency tests for finished product specifications is presented. Specific release and rejection criteria are defined particularly for the use of MSC for immunosuppressive applications. We suggest that cryopreserved MSC should be preferred for the use in clinical trials as the cell loss due to cryopreservation is outweighed by the possibility of extensive recipient-specific testing which will enhance the relevance of the study data.

Disclosure: No conflict of interest disclosed.
P869

\section{Impact of individual platelet lysates on isolation and growth of human mesenchymal stromal cells}

Horn, P.1; Bokermann, G. ${ }^{2}$; Cholewa, D. ${ }^{2}$; Bork, S. ${ }^{1}$; Walenda, T. ${ }^{2}$; Koch, C. ${ }^{2}$; Drescher, W. ${ }^{3}$; Hutschenreuther, G. ${ }^{4}$; Zenke, M. ${ }^{2}$, Ho, A.D. ${ }^{1}$; Wagner, W. ${ }^{2}$

${ }^{1}$ University of Heidelberg, Department of Medicine V, Heidelberg, Germany, ${ }^{2}$ Aachen University Medical School, Helmholtz-Institute for Biomedical Engineering, Aachen, Germany, ${ }^{3}$ Aachen University Medical School, Department for Orthopedics, Aachen, Germany, ${ }^{4}$ Aachen University Medical School, Institute for Transfusion Medicine, Aachen, Germany

Introduction: Culture medium for mesenchymal stromal cells (MSC) is frequently supplemented with fetal calf serum (FCS). FCS can induce xenogeneic immune reactions, transmit bovine pathogens and has a high lot-to-lot variability that hampers reproducibility of results. Human supplements have been used as substitutes for FCS before and several other groups demonstrated that pooled human platelet lysate (HPL) provides an attractive alternative. However, the composition of heterogeneous subpopulations might be affected by serum supplements and lot-to-lot variation of individual platelet lysates has not yet been addressed in detail.

Methods: In this study we compared the impact of serum supplements on initial fibroblastoid colony forming units (CFU-F). Subsequently, we addressed the activity of lysates from platelet units of individual donors on proliferation, in vitro differentiation and long-term culture of MSC. These data were correlated with chemokine profiles of HPLs.

Results: Isolation of MSC with either HPL or FCS resulted in similar CFU-F frequency, colony morphology, immunophenotype, and adipogenic differentiation potential. Osteogenic differentiation was more pronounced in HPL than in FCS. There was some variation in MSC proliferation with individual lysates but it was always higher in comparison to FCS. Proliferation of MSC correlated with the concentration of platelet-derived growth factor (PDGF) and there was a moderate association with platelet counts. All HPLs facilitated expansion for more than 20 population doublings.

Conclusions: Reliable long-term expansion was possible with each lysate of individual platelet units and this supports the notion that donor recipient matched or autologous HPL can be used for therapeutic MSC products. However, there was some variation in growth supportive potential and this correlated with PDGF concentration.

Disclosure: No conflict of interest disclosed.

\section{P870}

\section{Peripheral blood stem cell mobilization after Bendamustin pretreatment in patients with Multiple Myeloma}

Pönisch, W. ${ }^{1}$; Wiesler, J. ${ }^{1}$; Leiblein, S. ${ }^{1}$; Edel, E. ${ }^{2}$; Al-Ali, H.K. ${ }^{1}$; Basara, N. ${ }^{1}$; Hegenbart, U. ${ }^{3}$; Heiß, C. ${ }^{3}$; Niederwieser, D. ${ }^{1}$; Goldschmidt, $\mathrm{H}^{3}$

${ }^{1}$ Universität Leipzig, Hämatologie/Onkologie, Leipzig, Germany, 2Universität Leipzig, Institut für Blutspende und Transfusionsmedizin, Leipzig, Germany, ${ }^{3}$ Universität Heidelberg, Hämatologie/Onkologie, Heidelberg, Germany

Introduction: The alkylating agent bendamustine has structural similarities to both alkylating agents and purine analogs and is non-crossresistant with alkylating agents and other drugs in vitro. Bendamustine is effective in the treatment of patients with multiple myeloma, but no data are available on stem cell toxicity and on stem cell mobilization after bendamustine treatment.

Material and methods: A retrospective analysis on peripheral blood stem cell mobilizations over a period of fifteen years was carried out in 56 patients with multiple myeloma after bendamustine pretreatment at the university hospital Leipzig and Heidelberg. Patients had a median age of 58 years (range, 31-72 years). The median number of bendamustine cycles was 3 (range $1-10$ ) and the cumulative dosis of bendamustine was between 120 and $2400 \mathrm{mg} / \mathrm{qm}$. The mobilization regimen was either cyclophosphamide $4 \mathrm{~g} / \mathrm{qm}(\mathrm{n}=33)$ or $7 \mathrm{~g} / \mathrm{qm}$ $(\mathrm{n}=4)$ followed by G-CSF $(2 \times 5 \mathrm{ug} / \mathrm{kg}$ s.c.). Alternative regimen such as CAD, CED, TCED and others were also used in the remaining patients. Apheresis 
was started with peripheral blood $\mathrm{CD} 34^{+}$counts of $>10 \times 10^{3}$ and the standard target $4 \times 10^{6} \mathrm{CD}^{2} 4^{+} / \mathrm{kg}$, with a minimal target of $2 \times 10^{6} \mathrm{CD} 34^{+} / \mathrm{kg}$.

Results: In 54 of the 56 patients, successful harvest was obtained. Only in two patients insufficient stem cell mobilization was observed. In 18 out of 54 patients $(33 \%)$ the target was reached with a single apharesis. The median number of apharesis was two with a range from 1 to 7 . The median CD34+ cell-count $/ \mathrm{kg}$ obtained was $5,5 \times 10^{6}$ (range $\left.1,7-20,4\right) / \mathrm{kg}$. Of the 56 patients, 52 had a successful transplant.

Conclusion: These are first results showing that mobilization of peripheral blood stem cells for autologous stem cell transplantation is feasible after pretreatment with bendamustine.

Disclosure: Wolfram Pönisch: Honoraria: Mundipharma, Celgene; Financing of Scientific Research: Mundipharma, Celgene

Hartmut Goldschmidt: No conflict of interest disclosed.

\section{P871}

\section{Plerixafor (Mozobil ${ }^{\mathrm{TM}}$ ) and large volume apheresis} improve the yield of hematopoietic progenitor cells in patients insufficiently mobilized by chemotherapy and G-CSF alone

\section{Humpe, A. ${ }^{1}$; Renders, U. ${ }^{1}$; Günther, A. ${ }^{1}$; Buwitt-Beckmann, U. ${ }^{1}$; Jung, N. ${ }^{1}$; Valerius, T. $^{1}$; Gramatzki, M. ${ }^{1}$ \\ ${ }^{1}$ University Hospital Schleswig-Holstein Campus Kiel, Division of Stem Cell and Immunotherapy, Kiel, Germany}

Introduction: In the majority of patients successful mobilization of hematopoietic progenitor cells (HPC) for autologous transplantation can be achieved by chemotherapy and G-CSF. In some patients HPC mobilization is suboptimal and alternatives are needed. A novel cytokine receptor blocker plerixafor (Mozobil ${ }^{\mathrm{TM}}$ ), an antagonist of the CXCR4 receptor, may be such an alternative. Here, a series of 7 patients is reported, who insufficiently mobilized HPC after chemotherapy and G-CSF induction. In these patients harvest of a transplantation dose by one apheresis appeared highly unlikely according to a predictive algorithm formerly published.

Methods: Four male and 3 female patients with a median age of 61 years (range: 54 - 69 years) and diagnosed with multiple myeloma $(\mathrm{n}=5)$ or NHL $(\mathrm{n}=2)$ received plerixafor $(240 \mu \mathrm{g} / \mathrm{kg}$ body weight $(\mathrm{bw}))$ subcutaneously 10 hours before apheresis, in addition to the chemotherapy and 5-10 $\mu \mathrm{g} / \mathrm{kg}$ bw G-CSF applied already for mobilization. The addition of plerixafor was dependent on a number of CD34+ cells in peripheral blood (PB) not sufficient for harvest of a transplantation dose in one apheresis according to the predictive algorithm.

Results: Four of the 7 patients were submitted to apheresis in attempt to harvest HPC and showed a median yield of $1.21 \times 10 \mathrm{E}+06 \mathrm{CD} 34+$ cells $/ \mathrm{kg}$ bw before plerixafor was added. The median number of CD34+ cells and of white blood cells (WBC) in PB in the morning before addition of plerixafor were 11/ $\mu \mathrm{L}(4-20 / \mu \mathrm{L})$ and $11,400 / \mu \mathrm{L}(2,900-19,800 / \mu \mathrm{L})$, respectively. In the next morning, the median number of CD34+ cells as well as the number of WBC significantly $(\mathrm{p}<0.05)$ increased to $44 / \mu \mathrm{L}(22-112 / \mu \mathrm{L})$ and $28,300 / \mu \mathrm{L}(7,700$ - 48,600/ $\mu \mathrm{L}$ ). The total PB volume was apheresed 4.3 times (median). The apheresis process now resulted in a significantly higher median number of CD34+ cells $/ \mathrm{kg}$ bw and graft of $5.57(4.35-12.77) \times 10 \mathrm{E}+06$. So far, 6 of the 7 patients were successfully transplanted after high dose chemotherapy with a median amount of 3.69x10E+06 CD34+ cells/kg bw. A WBC count $>1 / \mathrm{nL}$ was reached after 11 days after transplantation (median).

Conclusions: Addition of plerixafor to chemotherapy and G-CSF mobilization together with a large volume leukapheresis was well tolerated and enabled for harvest of HPC numbers sufficient for transplantation after high dose chemotherapy. A transplantation dose together with a "back-up" graft could be harvested with one apheresis in formerly not optimally mobilizing patients.

Disclosure: Andreas Humpe: Honoraria: Geplante Vortragstätigkeit beim DGHO Jahreskongress 2010 für Genzyme; Other Financial Relationships: Geplante Übernahme der Reisekosten und Kongressteilnahme beim DGHO Jahreskongress 2010 durch Genzyme

Martin Gramatzki: No conflict of interest disclosed.

\section{Posterdiskussion Stammzelltransplantation experimentell \\ P872 \\ Reduced alloreactivity of NK cells after G-SCF mobilization in stem cell donors}

Kordelas, L. ${ }^{1}$; Switala, M. ${ }^{2}$; Heinemann, F.M. ${ }^{2}$; Ottinger, H. ${ }^{1}$; Beelen, D.W. ${ }^{1}$; Horn, P.A. ${ }^{2}$; Rebmann, V. ${ }^{2}$

${ }^{1}$ University Hospital Essen, Department of Bone Marrow Transplantation, Essen, Germany, ${ }^{2}$ University Hospital Essen, Institute for Transfusion Medicine, Essen, Germany

Introduction: Granulocyte colony-stimulating factor (G-CSF) is commonly used for stem cell mobilization in the context of autologous or allogeneic stem cell transplantation. A limited number of studies elucidating the effect of G-SCF on the specific leukocyte subtypes of the donor has been published so far. Earlier studies indicate a down-regulating effect on T cell activity. Little is known, in contrast, of the effect of G-CSF on NK cells. Therefore we investigated the cytotoxic function of NK cells derived from 13 peripheral stem cell donors before and 5 days after G-CSF administration.

Methods: NK cells were isolated from IL-2 stimulated peripheral blood cells by negative selection. NK cell cytotoxicity was determined by flow cytometry using the HLA-E*01:03 transfected K562 cells and the parental HLA class I negative chronic myelogenous leukemia K562 cell line as target cells in a effector/target ratio of 6:1. Lysed target cells were detected by propidium iodide staining. In addition, the expression of HLA-E specific receptors (CD94, NKG2A and C) was analysed on NK cells from 10 donors before and after G-CSF treatment by flow cytometry.

Results: The NK cells procured after G-CSF administration showed a reduced cytotoxicity (mean \pm SEM \%) towards HLA class I negative K562 target cells $(10.4 \pm 3.3)$ compared to NK cells obtained before G-CSF treatment $(5.3 \pm$ 1.6, $\mathrm{p}=0.06)$. This effect was found to be more prominent in HLA-E*01:03 transfected K562 target cells $(10.9 \pm 3.7$ vs. $2.8 \pm 1.3 ; \mathrm{p}=0.03)$. The reduced capability to lyse HLA-E*01:03 transfected K562 target cells can be explained by the reason that the proportion of NK cells bearing HLA-E specific receptors were significantly decreased after G-CSF administration. The proportion of CD94, the inhibitory receptor NKG2A and the activating receptor NKG2C on NK cells before and after G-CSF were as followed: CD94: $66.4 \pm 4.3$ vs. 57.7 $\pm 4.9 ;$ NKG2A: $46.7 \pm 4.9$ vs. $43.7 \pm 6.1 ;$ NKG2C: $8.1 \pm 4.5$ vs. $3.4 \pm 1.3$.

Conclusion: To the best of our knowledge this study is the first one showing that G-CSF decreases the proportion of NK cells with HLA-E specific receptors, which in turn results in a reduced lytic function towards HLA-E* 01:03 positive target cells. A prospective study needs to investigate how the expression of other NK cell receptors is influenced by G-CSF.

This study was supported by a research grant by the Deutsche José Carreras Leukämie-Stiftung e.V.

Disclosure: No conflict of interest disclosed.

\section{P873}

\section{Effect of G-CSF mobilization on the release of soluble NKG2D ligands in stem cell donors}

Rebmann, V. ${ }^{1}$; Switala, M. ${ }^{1}$; Lindemann, M. ${ }^{1}$; Horn, P.A. ${ }^{1}$; Beelen, D.W. ${ }^{2}$; Kordelas, $L^{2}{ }^{2}$

${ }^{1}$ University Hospital Essen, Institute for Transfusion Medicine, Essen, Germany, ${ }^{2}$ University Hospital Essen, Department of Bone Marrow Transplantation, Essen, Germany

Introduction: Ligands of the natural killer group 2 member D (NKG2D) receptor play a specific role in several immune pathways. Surface expressed NKG2D ligands (NKG2DL) such as MICA, MICB, and ULBP2 trigger NK cells and costimulate T lymphocytes whereas soluble NKG2DL (sNKGDL) impairs the effector function of these cells. Soluble molecules of NKG2DL are generated either by shedding, proteolysis or by the release of microvesicles from NKG2DL positive cells. As granulocyte colony-stimulating factor (G-CSF) - a cytokine frequently used for stem cell mobilization - impairs the effector function of lymphocytes we question whether G-CSF can mediate the release of soluble MICA (sMICA), sMICB, and sULBP2. 
Methods: We analysed sMICA/B and sULBP2 levels in samples procured from 33 peripheral blood stem cell (PBSC) donors before and 5 days after G-CSF administration by specific ELISAs. In 3 patients the course of sNKG2DL levels were serially monitored once a month in the first 6 months after allogeneic SCT. Microvesicle or protein aggregate formations of sNKG2DL with soluble TIE-2 molecules - a receptor expressed by endothelial cells or early hematopoietic cells - were detected by the combination of antibodies specific for NKG2DL and TIE-2.

Results: Levels of sNKG2DL (mean \pm SEM pg/ml) were significantly increased $(\mathrm{p}<0.01)$ in plasma samples procured after G-CSF administration (sMICA: 543 \pm 88 ; sMICB: $181 \pm 32$; sULBP2: $199 \pm 33$ ) compared to samples obtained before G-CSF treatment (sMICA: $387 \pm 66$; sMICB: $84 \pm 23$; sULBP2: $79 \pm 18$ ). The detection of microvesicle or protein aggregate formations positive for both sNKG2DL and TIE-2 molecules suggests that sNKG2DL molecules were originated from endothelial cells or early hematopoietic cells. In two of the three patients no sNKG2DL was detected after allogeneic SCT. The only patient with a substantial amount of sNKG2DL experienced acute graft versus host disease.

Conclusion: To the best of our knowledge this study is the first to show that G-CSF mediates the release of sNKG2DL/TIE-2 positive microvesicles or protein aggregate formations. This observation strongly suggests that sNKG2DL molecules are originated by endothelial cells or early hematopoietic cells. Further studies will have to investigate the functional and clinical relevance of such vesicle/complex formations for allogeneic stem cell transplantation.

This study was supported by a research grant by the Deutsche José Carreras Leukämie-Stiftung e.V.

Disclosure: No conflict of interest disclosed.

\section{P874}

Steroid treatment alters adhesion molecule and chemokine expression in experimental acute graft versus host disease of the intestinal tract

Bouazzaoui, A. ${ }^{1}$; Spacenko, E. ${ }^{1}$; Muller, G. ${ }^{1} ;$ Huber, E. ${ }^{2}$; Schubert, T.; Holler, E. ${ }^{3}$; Andreesen, R. ${ }^{1}$; Hildebrandt, G.C. ${ }^{1,4}$

${ }^{1}$ Klinik Uni-Regensburg, Häma/Onko, Regensburg, Germany, ${ }^{2}$ Klinik Uni-Regensburg, Pathology, Regensburg, Germany, ${ }^{3}$ Germany Institut of Applied Pathology, Speyer, Germany, ${ }^{4}$ Feist-Weiler Cancer Center, Blood and Marrow Transplant Program, Shreveport, United States

Acute graft versus host disease (aGVHD) is a major complication after allogeneic bone marrow transplantation (allo-BMT) characterised by high morbidity and mortality. Systemic treatment with steroids has been the mainstay of first line therapy of aGVHD, although controlled experimental data in this context are limited. Using a haploidentical murine BMT model, the steroid effects on hepatic and intestinal inflammation during aGVHD have been investigated. Lethally irradiated B6D2F1 mice received $5 \times 10^{6}$ bone marrow cells supplemented with $10 \times 10^{6}$ splenocytes from either syngeneic (B6D2F1) or allogeneic (C57BL/6) donors. Intraperitoneal administration of prednisolone $(2 \mathrm{mg} / \mathrm{kg}$ BW qd) from day 10 until day 42 resulted in reduced clinical GVHD severity and improved survival of allogeneic recipients, along with decreased alloantigen-specific $\mathrm{T}$ cell expansion in vivo and in vitro. Interestingly, while aGVHD of the liver was barely affected following prednisolone treatment, aGvHD-related histopathologic injury of the GI tract was strongly reduced in association with diminished expression of INFg, TNF, of inflammatory chemokines including CXCL 9-11, CCL2-4 and of MadCAM-1 and ICAM-1. Even more, prednisolone-induced reduction of adhesion molecule expression in the gut manifested earlier than seen for cytokines or chemokines.

Conclusion: Steroid-mediated beneficial effects in the treatment of GVHD affect organs differently depending on anatomical structures such as Peyer's patches, differences in T cell subpopulations or divergent regulation of inflammation and leukocyte migration. Specifically, a change in adhesion properties between leukocytes and endothelial cells in the GI tract may be one of the initial steps in a steroid-mediated cascade of aGVHD-attenuating events.

Disclosure: No conflict of interest disclosed.

\section{P875}

\section{Primary reconstitution of human antibody secreting cells after B-cell and T-cell depleted allogeneic stem cell transplantation}

\author{
Steurer, M. ${ }^{1}$; Malenke, E. ${ }^{1}$; Federmann, B. ${ }^{1}$; Kanz, L. ${ }^{1}$; \\ Bethge, W. ${ }^{1}$; Wirths, S. ${ }^{1}$
}

${ }^{1}$ Universität Tübingen, Department Innere Medizin, Hämatologie, Onkologie, Rheumatologie, Immunologie, Pulmologie, Tübingen, Germany

Introduction: Reconstitution of adaptive immunity after allogeneic stem cell transplantation is frequently delayed and incomplete over months. Innate immunity elements including granulocytes, monocytes, and NK cells in contrast are reported to recover rapidly within weeks. Kinetics in reconstitution of innate and adaptive immunity after ablation in adults may give insight into hierarchy and attribution to innate and adaptive immunity of defined lymphocyte populations. In murine models innate type marginal zone and B1 B cells, established at fetal age and providing natural antibodies, are distinguished from adaptive B2 cells. A crucial maturation and survival factor for adaptive murine B cells was shown to be the TNF-family member BAFF (B cell-activating factor), while development of innate type B1 B cells is BAFF independent.

Methods: Reconstitution of lymphopoiesis after CD3 and CD19 depleted haploidentical stem cell transplantation was analyzed in 10 patients, which received OKT3 as immunosuppressant only. Weekly samples before and after reduced-intensity conditioning were analyzed by flow cytometry for absolute numbers of T-cell, NK-, and B-cell subsets. Their origin of host or donor hematopoiesis was differentiated by HLA-surface expression. Antibody secreting cells (ASC) were enumerated by ELISPOT. Masquerade of myeloid cells was excluded by kappa/lambda two-color ELISPOT to demonstrate light chain restriction.

Results: CD19+ B-lymphocytes of naïve and memory phenotype (>0,5\% of all lymphocytes) were not detected before day +60 . In contrast, complete reconstitution of antibody-secreting cells after a nadir $(<0,05 / \mu \mathrm{l})$ was observed at day +14 . Absolute numbers of ASC were even higher than those of healthy controls (d+14: $72 / \mu \mathrm{l}$ vs. control: $12 / \mu \mathrm{l})$, and ASC secreting the isotypes IgM and IgA were more prevalent than IgG compared to controls (IgM 20 fold; IgA 10 fold; IgG 3 fold). Reconstitution of ASC occurred without detectable circulating T-cells and before an increase of BAFF concentration was observed. Conclusion: The rapid and complete reconstitution of peripheral blood ASC after allogeneic transplantation, far proceeding detection of naïve and memory type B-cells, is a novel observation. Incidence before T-cell reconstitution and increase in BAFF concentrations indicates a T-cell and BAFF independent mechanism allocating these early ASC to innate immunity, potentially maintaining natural antibody levels.

Disclosure: No conflict of interest disclosed.

\section{P876}

The role of $\mathrm{Vd} 1$ positive gdT-cells in controlling CMVreactivation after allogeneic stem cell transplantation

\section{van Dorp, S. ${ }^{1}$; Pietersma, F. ${ }^{1}$; Hol, S. ${ }^{1}$; Heijhuurs, S. ${ }^{1}$;} Marcu-Malina, V. ${ }^{1}$; Minnema, M. ${ }^{1}$; Becke, S. ${ }^{2}$; Plachter, B. ${ }^{1}$; van Baarle, D. ${ }^{1} ;$ Kuball, J. ${ }^{1}$

${ }^{1}$ UMC Utrecht, Utrecht, Netherlands, ${ }^{2}$ UMC Mainz, Mainz, Germany

One major problem after allogeneic-stem cell transplantation (allo-SCT) remains a severe CMV-infection. V $\delta 2^{\text {neg }} \gamma \delta \mathrm{T}$-cells have been reported to be involved in controlling CMV-disease after solid organ transplantation. Therefore, we investigated the potential role of $\gamma \delta \mathrm{T}$-cells after allo-SCT in controlling CMV-disease and to cross-talk with the adaptive immune system. Frequency and function of $\gamma \delta \mathrm{T}$-cells were investigated in the graft and patients after alloSCT. No correlation could be observed between the frequency of total gdT-cells in the graft and leukemia relapse, CMV-reactivation or graft-versus-host-disease (GVHD). However, CMV-reactivation after allo-SCT induced a specific expansion of $\mathrm{V} \delta 2^{\text {neg }} \mathrm{gdT}$-cells of donor origin. In addition, in vivo-primed $\mathrm{V} \delta 2^{\text {neg }} \mathrm{gdT}-$ cells were reactive against CMV-infected fibroblasts. Within the V $82^{\text {neg }}$ gdT-cell-fraction, V $\delta 1^{\text {pos }}$ gdT-cells were CMV-reactive and up-regulated CD137 upon stimulation with CMV-infected fibroblasts. Furthermore, in vivo-primed 
$\mathrm{V} \delta 2^{\text {neg }} \mathrm{gdT}$-cells were able to mature dendritic cells in a CMV-independent way via CD1c which could play an important role in supporting a CMV-specific adaptive immune-response. As V $\delta 1^{\text {pos }}$ gdT-cells could be detected not only in the graft of healthy donors, but also in the graft of CMV-negative donors, V $\delta 1^{\text {pos }}$ gdT-cells can serve as an alternative source of CMV-reactive T-cells which might be useful in order to prevent or treat CMV disease in patients with CMV-negative donors after allo-SCT.

Disclosure: No conflict of interest disclosed.

\section{P877}

\section{Mechanisms of regulatory T cell-mediated suppression of alloreactivity in the context of acute graft-versus-host disease}

\author{
Lupp, C. ${ }^{1}$; Bopp, T. ${ }^{1}$; Schmitt, E. ${ }^{1}$; Schild, H. ${ }^{1}$; Radsak, M.1,2 \\ ${ }^{1}$ Universitätsmedizin der Johannes Gutenberg-Universität, Institut für \\ Immunologie, Mainz, Germany, ${ }^{2}$ III. Medizinische Klinik der Johannes \\ Gutenberg-Universität, Mainz, Germany
}

Allogenic bone marrow transplantations are the only curative treatmen options for patients with relapsed or high risk leukemias or lymphomas. The recognition of host alloantigens by donor $\mathrm{T}$ cells is most likely the basis of graft-versus-tumor effects, but is oftentimes also the cause of graft-versus-hos disease, a key contributor to the high morbidity and mortality rates. Regulatory FoxP $3^{+} \mathrm{CD}^{+}{ }^{+} \mathrm{T}$ cells (Tregs) are known to suppress the activation of conventional $\mathrm{T}$ cells (e.g. $\mathrm{CD}^{+}$or $\mathrm{CD} 4^{+} \mathrm{T}$ cells) and represent a therapeutic paradigm for the control of GvHD.

In our present study we analyzed potential mechanisms to prevent or attenuate acute graft-versus-host reactions in an allogenic MHC mismatched transplantation model.

We performed allogenic mixed lymphocyte reactions (MLR) in vitro and allogenic bone marrow transplantations in mice to study the suppressive effects of Tregs. Lethally irradiated BALB/c mice were transplanted with bone marrow and CD $90.2^{+} \mathrm{T}$ cells from C57BL/6 donors resulting in severe graft-versus-host disease. Additional transplantation of donor $\mathrm{CD} 4^{+} \mathrm{CD} 25^{+}$Tregs rescued the animals from GvHD. In vitro and in vivo studies using Tregs from IL-10 deficient donors demonstrated that Treg-mediated suppression of alloresponses occurred in an IL-10 independent manner. Further in vitro studies showed that Tregs communicate with allogenic dendritic cells (DCs) via direct gap junction intercellular communication, demonstrated by the transfer of calcein from calcein-labeled Tregs to DCs. Tregs suppressed the activation of allogenic DCs cells by reducing the upregulation of costimulatory molecules (CD80 / CD86). In conclusion, our results demonstrate that Tregs interact with allogenic antigen presenting cells via gap junction intercellular communication while Treg derived IL-10 seems to be dispensable for the inhibition of alloreactivity. These data provide the basis for future concepts to manipulate allogenic $\mathrm{T}$ cell responses to prevent GvHD.

Disclosure: No conflict of interest disclosed.

\section{P878}

\section{Reliability of quantitative hematopoietic chimerism testing, assessment of performance and error quantification}

Waterhouse, M. ${ }^{1}$; Huber, I. ${ }^{1}$; Samek, E. ${ }^{1}$; Enger, S. ${ }^{1}$; Riedl, M. ${ }^{1}$; Bertz, H. ${ }^{1}$; Finke, J. ${ }^{1}$

${ }^{1}$ Universitätsklinikum Freiburg, Hematologie/Onkologie, Freiburg, Germany

Quantitative hematopoietic chimerism testing is a key element for the follow up of patients after allogeneic hematopoietic cell transplantation (HCT) Chimerism testing commonly relies on an assay for short tandem DNA repeats (STRs). DNA content from patient and recipient in the clinical sample is estimated using relative fluorescence units (RFU) and elapsed time of retention. Hence, the amount of DNA at an allele is not directly measured but only estimated from indirect measurements that correlate with quantity. Therefore an unavoidable systematic error is introduced in the allele quantification by STRs. Our goal was to quantify the introduced error and analyze the analytical impact in chimerism testing after allogeneic HCT. For this purpose artificial mixed chimeras using peripheral blood cells from unrelated individuals, were made. Clinical samples from transplanted patients, with different levels of hematopoietic chimerism, were used as well. STRs for different markers showed an excellent correlation with FISH and amelogenin (non STR, PCR based method). Correlation coefficient was $>0.90$, both with artificially created chimeras and with clinical samples in all levels of chimerism tested $(0-100 \%)$. Lowest analytical sensitivity was $5 \%$, while FISH and amelogenin showed a sensitivity of $0.5 \%$. Limit of detection (LD) for all STRs was determined as 3 times the standard deviation of the mean RFU for each STR. The highest limit of detection was detected in THO1 marker with a RFU of 200. In order to detect the error introduced in STRs determination donor and patient's allelic ratio difference was measured at different levels of chimerism $(1,5,10$, 20 and $50 \%$ ). Low allelic peak ratio resulted in up to $50 \%$ difference in the chimerism percentage. To conclude there is a significant correlation STRs and FISH or non STRs (amelogenin) both in artificial chimeras and clinical samples. Low peaks (under LD) should be interpreted with caution because there is a significant probability that there are parts of background RFU. Suboptimal PCR performance (low allelic ratio) has clear impact in chimerism testing, with potential clinical consequences.

Disclosure: No conflict of interest disclosed.

\section{P879}

\section{The dynamics of homing receptor expression on human peripheral blood T cells}

Herz, S. ${ }^{1}$; Bäuerlein, C. ${ }^{1}$; Jordán Garotte, A.-L. ${ }^{1}$; Riedel, S. S. ${ }^{1}$; Brede, C. ${ }^{1}$; Kiesel, C. ${ }^{1}$; Ritz, M. ${ }^{1}$; Chatterjee, M. ${ }^{1}$; Negrin, R.S. ${ }^{2}$; Einsele, H. ${ }^{1}$; Beilhack, $A{ }^{1}$

${ }^{1}$ Universitätsklinikum Würzburg, Medizinische Klinik und Poliklinik II, Würzburg, Germany, ${ }^{2}$ Stanford University, Division of Blood and Marrow Transplantation - Department of Medicine, Stanford, United States

Acute graft-versus-host disease (aGvHD) poses a major risk of allogeneic hematopoietic cell transplantation (allo-HCT). In murine in vivo imaging models we recently demonstrated that aGvHD pathogenesis is tightly spatially and temporally regulated. We showed that alloreactive $\mathrm{T}$ cells need to express certain homing receptors in order to reach the aGvHD target organs (intestinal tract, liver and skin). Thus, we reasoned that certain homing receptors on peripheral blood $\mathrm{T}$ cells could serve as predictive markers for aGvHD. This could improve the prevention and treatment of aGvHD.

To establish a clinical test to predict aGvHD we developed a multiplex flow cytometry test for cytotoxic and $\mathrm{T}$ helper cell subsets. We investigated the expression of 25 activation markers, organ specific homing receptors and inflammatory response receptors on human peripheral blood $\mathrm{T}$ cells that possibly play a role in the pathogenesis of aGvHD in patients. Here we evaluated how $\mathrm{T}$ cell surface marker expression changes over time in healthy probands. For our study we recruited 22 healthy individuals (female and male, mean age 24,9 years). We analyzed peripheral blood mononuclear cells by flow cytometry at 8 time points within 3 weeks. Preliminary mouse experiments in our group had determined these time points as critical for subsequent prospective clinical studies.

We found that the surface marker expression on peripheral blood cytotoxic and $\mathrm{T}$ helper cells in healthy probands can be subdivided into 3 categories:

1. Constant low numbers of cytotoxic and T helper cells expressed CD25, CD69, chemokine receptor (CCR) 2, CCR10, CXCR1 (on T helper cells only), CXCR2, cutaneous lymphocyte antigen, leukotriene B4 receptor 1 and integrin alpha E.

2. Constant high numbers of $\mathrm{T}$ cells expressed CD45RA, CD62L, integrin alpha 4.

3. T cell subsets that dynamically changed the expression of CCR1, CCR4, CCR5, CCR6, CCR8, CCR9, CXCR1 (on cytotoxic T cells only), CXCR3 and integrin beta 7 .

With the data from healthy probands we established the basis for a recently initiated clinical prospective study. The analyzed homing receptor profiles were important to determine physiological ranges to compare them to alloHCT patients. The data from the healthy individuals encourage further investigations with allo-HCT patients to find an efficient and reliable method to predict aGvHD.

Disclosure: No conflict of interest disclosed. 
P880

Soluble Human Leukocyte Antigen G (sHLA-G) and 14 bp polymorphisms in HLA G gene are associated with several clinical complications after allogeneic hematopoietic cell transplantation

Waterhouse, M. ${ }^{1}$; Duque-Afonso, J. ${ }^{1}$; Samek, E. ${ }^{1}$; Bertz, H. ${ }^{1}$; Finke, J. ${ }^{1}$

${ }^{1}$ Universitätsklinikum Freiburg, Hematologie/Onkologie, Freiburg, Germany

HLA-G is a non classical class I HLA molecule with low level of polymorphisms and with 7 isoforms, 4 are membrane bound and 3 are soluble. Another differential characteristic is it is tissue restricted and have been described in adult thymic medulla, cornea, pancreatic islet and endothelial cell precursors. Several immune modulatory functions have been attributed to this molecule such as the interaction between B, T, NK and antigen presenting cells. Due to their immunomodulatory properties we investigated the possible role of sHLA-G and the 14 bp polymorphisms of HLA G gene in the allogeneic hematopoietic cell transplantation (HCT) setting. A cohort of 59 patients, who underwent HCT, was studied, 22 patients had acute myeloid leukemia, and 11 patients had myelodisplastic syndromes, while the rest of the patients had non myeloid malignancies.

Patients received mostly reduce intensity conditioning regimen $(97 \%)$. DNA and plasma samples from all patients were obtained before the conditioning regimen and after transplant at different time points. Patients with the $+14 /+14$ polymorphism in exon 8 had significant lower sHLA G plasmatic levels. We found a statistically significant association between pre-transplant low levels of sHLA G, +14/+14 polymorphism and infectious complications, in particular fungal infections. Low pre transplant sHLA G levels were associated with increased mortality after transplant. Interestingly no relapse was detected among patients with the $+14 /+14$ genotype. Our data suggest that sHLA-G molecules and exon 8 polymorphism in the HLA G gene seems to play a role in several complications after allogeneic hematopoietic cell transplantation.

Disclosure: No conflict of interest disclosed.

\section{P881}

SNP array analysis in allogeneic transplanted patients reveals several genomic lesions and acquired uniparental disomy

Waterhouse, M. ${ }^{1}$; Pfeifer, D. ${ }^{1}$; Pantic, M. ${ }^{1}$; Zerweck, A. ${ }^{1}$; Bertz, H. ${ }^{1}$; Finke, J. ${ }^{1}$

${ }^{1}$ Universitätsklinikum Freiburg, Hematologie/Onkologie, Freiburg, Germany

Hematological neoplasm can be effectively treated with allogeneic hematopoietic cell transplantation (HCT). Although relapse is one of the main causes of treatment failure.

Cytogenetics studies stratify patients into three groups of prognosis according to the overall survival but these classifications are not fully predictive of response. SNP array analysis allows a detailed detection of genomic changes in patients undergoing allogeneic HCT. We performed high resolution singlenucleotide polymorphism array-profiling in 19 allogeneic HCT patients, before HCT and at relapse after HCT in paired matched samples. Primary acute myeloid leukemia (AML) or myelodisplastic syndrome (MDS) was diagnosed in 17 patients, while the remaining 2 patients had lymphoid malignancies. In five out of six patients with a normal karyotype pre-transplant, genomic aberrations (GA) was a novel finding. GA were present in the form of gains and losses (5/1 gains/losses) in several regions of different chromosomes with a median of 2 GA per case. The abnormal karyotype group, in addition to the cytogenetically detected aberrations, GA were present in 5 out of 13 patients (7/6 gains/ losses). All FAB subtypes of AML showed GA, being M5 and secondary AML both with more numerous GA (average: 12 and 17 GA respectively). In the relapse sample all patients showed GA in several chromosomes. Recurrent GA were present in chromosomes 9 (q22.31), 16 (q13), 19 (p13.11) and 22 (q11.23). Loss of heterozygosity (LOH) was found in chromosomes $3,4,6,10,11,13,14,16$ and 18. All LOH were more than $10 \mathrm{mb}$ and some of them involved the whole chromosome arm. Interestingly LOH were found in chromosome $6 \mathrm{p}$ in which HLA genes are encoded and in chromosome 9p were JAK 2 gene is located. LOH in chromosome 9p resulted in homozygosity for JAK2 mutation detected by pyrosequencing. GA were validated using FISH and biostatistical evaluation of the array data. No significant association was found between GA and $\mathrm{LOH}$ detected by SNP array and clinical outcome. Genome wide profiling should be taken in account for risk stratification in patients undergoing allogeneic HCT.

Disclosure: No conflict of interest disclosed.

\section{P882}

\section{Increase in uric acid serum level indicates incipient release of leukocytes after allogeneic stem cell transplantation}

Haen, S.P. ${ }^{1}$; Eyb, V. ${ }^{1}$; Kanz, L. ${ }^{1}$; Rammensee, H.-G. ${ }^{2}$; Bethge, W. ${ }^{1}$ ${ }^{1}$ Medizinische Universitätsklinik Tübingen, Abteilung II, Tübingen, Germany, ${ }^{2}$ Interfakultäres Institut für Zellbiologie, Abteilung Immunologie, Tübingen, Germany

Introduction: Uric acid is released from dying cells and serves as an endogenous danger signal to the immune system activating both innate and adaptive immunity. During haematopoietic cell transplantation (HCT) multiple cytokines and danger signals are released into the blood modulating cellular and humoral immune responses. Uric acid levels may be elevated during engraftment after HCT due to consumption of early recurring differentiated cells in the periphery. We investigated serum levels of uric acid during aplasia after allogeneic HCT to evaluate its use as early marker of engraftment activity before detection of leukocytes in differential blood counts.

Methods: We retrospectively analyzed differential blood counts and serum levels of uric acid, creatinine and urea in 47 consecutive patients ( 22 men and 25 women, median age 48 years, range 19 to 73 years) during 50 allogeneic HCT (three patients received a second transplantation due to recurrent disease). All patients received allopurinole for 5 days from the beginning of the conditioning which was discontinued afterwards. Serum levels of uric acid and peripheral blood counts were determined daily.

Results: The mean nadir in uric acid levels was observed 13 days (median) after the last dose of allopurinole (range 2 to 32 days) and 9 days after transplantation (range 2 to 21 days). These time differences were independent from the conditioning regimen. Earliest leukocytes $(>50 / \mu \mathrm{L})$ were detected between day 6 and 22 (median day 11, SD 3.8) while hematopoietic engraftment (neutrophils $>500 / \mu \mathrm{l}$ ) occurred between day 10 and 43 (median day 18, SD 6.1). After 35 out of 50 transplantations, a re-increase of uric acid was observed 1 to 10 days (median 4 days, SD 2.4) before detection of leukocytes in peripheral blood. In three cases, the rise of uric acid levels was observed concomitantly with increasing leukocytes. In the remaining 12 cases, this elevation was observed later (increase 1 to 6 days after re-increase of leukocytes, median 2 days, SD 1.5). The difference between timepoints of uric acid rise in the first and the last group was statistically significant (Student's t-test, $\mathrm{p}<0.001)$.

Conclusion: A re-increase of uric acid can be indicative for incipient haematopoietic reconstitution after allogeneic HCT and may serve as early indicator for engraftment activity even before leukocytes can be detected in the blood.

Disclosure: No conflict of interest disclosed.

P883

Immature platelet fraction as a predictor for thrombopoiesis in children after stem cell transplantation?

Hennel, E.K. ${ }^{1}$; Böer, K. ${ }^{1}$; Kiehntopf, M. ${ }^{1}$; Beck, J.F. ${ }^{2}$; Kentouche, $K^{2}$

${ }^{1}$ Universitätsklinikum Jena, Institut für Klinische Chemie und Laboratoriumsmedizin, Jena, Germany, ${ }^{2}$ Universitätsklinikum Jena, Klinik für Kinder- und Jugendmedizin, Jena, Germany

Introduction: Immature platelet fraction (IPF) correlates with megacaryocyte activity and is discussed as a predictor of platelet regeneration after stem cell transplantation (SCT) in adult patients to reduce platelet transfusions (Briggs et al.). So far, only one study has monitored IPF in children. (Saigo et al. 
Table 1. Results (for Abstract P883)

\begin{tabular}{|l|l|l|l|}
\hline & $\begin{array}{l}\text { group A: } \\
\text { IPF } \max >10 \% \mathrm{n}=8\end{array}$ & $\begin{array}{l}\text { group B: } \\
\text { IPF } \max 6-10 \% \mathrm{n}=6\end{array}$ \\
\hline age $(\mathrm{m}=$ months, $\mathrm{y}=$ years) & $6 \mathrm{~m}-18 \mathrm{y}$ & $19 \mathrm{~m}-18 \mathrm{y}$ & \\
\hline mean IPF \% (range) & $17.6(10.2-30.0)$ & $8.5(7.9-9.2)$ & $25 \mathrm{~m}-9 \mathrm{y}$ \\
\hline days between IPF peak and platelet recovery & $6(1->10)$ & $5.7(2-16)$ & $5.7(3.5-5.8)$ \\
\hline
\end{tabular}

2007). We investigated if IPF could predict the onset of thrombopoiesis in children after SCT.

Patients and methods: 16 paediatric patients undergoing stem cell therapy were enrolled in this study. Platelets and IPF were continuously measured; clinical parameters and the need for platelet concentrates were monitored.

Results: Despite an increase of IPF in all patients it was not always followed by a rise of platelets during a defined time. Overall sensitivity for a platelet rise within 3 days was 1 for an IPF cut-off of $6 \%$ and 0,4 for an IPF cut-off of $10 \%$. Specificity was 0.18 resp. 0.45 .

Conclusion: Even high IPF $>10 \%$ were only followed by a platelet recovery within 3 days in 2 of 8 patients. Infections were described to cause an increase of IPF not followed by increasing platelet counts (Briggs 2004) but patient histories did not suggest this causality. Interestingly the opposite occurred, namely peek IPF counts in 4 patients were associated with no instant platelet recovery even after 10 days. IPF should not be regarded as a reliable marker for the onset of thrombopoiesis after SCT in children.

References:

Briggs et al., Assessment of an immature platelet fraction (IPF) in peripheral thrombocytopenia. Br J Haematology 2004; 126: 93-99

Saigo et al., Automatic detection of immature platelets for decision making regarding platelet transfusion indications for pediatric patients, Transfusion and Apheresis Science 2008; 38, 127-132

Disclosure: No conflict of interest disclosed.

\section{Posterdiskussion}

Tumore Magen, Pankreas, Leber

P884

\section{Expression of VEGFR-1 is associated with bone marrow} micrometastasis in esophageal carcinoma

Schultze, A. ${ }^{1}$; Bubenheim, M.2; Yekebas, E. ${ }^{3}$; Erbersdobler, A.4; Reichelt, U. ${ }^{4}$; Izbicki, J.R. ${ }^{5}$; Pantel, K. ${ }^{6}$; Bokemeyer, C. ${ }^{1}$; Fiedler, $W^{1}{ }^{1}$; Loges, S. $^{1,6}$

${ }^{1}$ Universitätsklinikum Hamburg-Eppendorf, Hämatologie und Onkologie mit den Sektionen KMT und Pulmologie, Hubertus Wald Tumorzentrum, Universitäres Comprehensive Cancer Center Hamburg, Hamburg,

Germany, ${ }^{2}$ Biostatistics Department; University of Rouen, Rouen, France, ${ }^{3}$ Klinik und Poliklinik für Allgemein-, Viszeral- und Thoraxchirurgie; Universitätsklinikum Hamburg-Eppendorf, Hamburg, Germany, ${ }^{4}$ Institut für Pathologie, Charité, Berlin, Germany, ${ }^{5}$ Klinik für Allgemein-, Visceral- und Thoraxchirurgie; Universitätsklinikum Hamburg-Eppendorf, Hamburg, Germany, ${ }^{6}$ Institut für Tumorbiologie, Universitätsklinikum HamburgEppendorf, Hamburg, Germany

Background: Placental growth factor (PlGF) and its receptor vascular endothelial growth factor receptor-1 (VEGFR-1) are involved in tumor progression. Humans can express four PlGF isoforms (PlGF1-4), but it is unknown, whether all of them are expressed in cancer. In this retrospective exploratory study, we analyzed expression levels of PIGF1-4 and of VEGFR-1 in primary esophageal tumor tissue and correlated gene expression data with clinical data. We included presence of disseminated tumor cells (DTCs) in the bone marrow in our analysis, which has recently evolved as adverse prognostic factor in different cancers.

Methods: Gene expression levels were determined by QRT-PCR in primary tumor samples of 69 patients with esophageal cancer. Subsequently, expression data was correlated with clinical data (pT, pN, pM, survival, DTCs). For analysis of association between expression levels and categorical clinical parameters Wilcoxon's test for independent samples was used. To determine correlation between quantitative variables, Kendall's tau (t) was used to measure the degree of dependence.

Results: PLGF-1, PLGF-2 and VEGFR-1 were expressed in virtually all analyzed tumor samples. Expression of PLGF-3 and PLGF-4 were limited to 59\% and $74 \%$ of the analyzed patients, respectively. Expression levels of all PlGF variants were correlated amongst each other (Kendall tau rank>0.55). Expression of PlGF splice variants was not correlated with clinical variables. Interestingly, expression levels of VEGFR-1 predict presence DTCs in bone marrow $(\mathrm{p}=0.002)$. Patients with DTC in bone marrow had lower expression levels of VEGFR-1 within primary tumor tissue than patients without DTCs $(0.004 \pm 0.01$ vs. $0.07 \pm 0.15)$. In contrast, VEGFR-1 expression level was not correlated with lymphatic or distant macrometastasis, indicating a potential specific role of VEGFR-1 in protecting from dissemination of tumor cells into then bone marrow.

Conclusions: All four known isoforms of PLGF are expressed in human esophageal cancer tissue. Furthermore, VEGFR-1 levels are inversely correlated with presence DTCs in the bone marrow of patients. If validated in prospective studies, VEGFR-1 expression in tumors might serve as novel prognostic biomarker for bone marrow micrometastasis.

Disclosure: No conflict of interest disclosed.

P885

Docetaxel, cisplatin and capecitabine (DCX) as perioperative chemotherapy in gastro-esophageal adenocarcinoma. (A phase II study of the Arbeitsgemeinschaft Internistische Onkologie AIO)

Gahn, B. ${ }^{1}$; Bichev, D. ${ }^{2}$; Hofheinz, R. ${ }^{3}$; Arnold, D. ${ }^{4}$; Florschütz, A. ${ }^{5}$ Daum, S. ${ }^{6}$; Kretzschmar, A. ${ }^{7}$; Mantovani-Löffler, L. ${ }^{8}$; Kneba, M. ${ }^{1}$; Reichardt, P. ${ }^{9}$; Schumacher, G. ${ }^{10}$; Thuss-Patience, P.C. ${ }^{2}$

${ }^{1}$ Universitätsklinikum Schleswig-Holstein, II. Medizinische Klinik, Kiel, Germany, ${ }^{2}$ Charité - Campus Virchow-Klinikum, Med. Klinik m.S. Hämatologie und Onkologie, Berlin, Germany, ${ }^{3}$ Universitätsmedizin Mannheim, Hämatologie und Internistische Onkologie, Mannheim, Germany, ${ }^{4}$ Martin Luther University Halle-Wittenberg, Universitätsklinik und Poliklinik für Innere Medizin IV, Halle, Germany, ${ }^{5}$ Städtisches Klinikum Dessau, Klinik f Innere Medizin, Hämatologie/Onkologie, Dessau, Germany, ${ }^{6}$ Charité - Campus Benjamin Franklin, Med. Klinik m.S. Gastroenterologie, Infektiologie u Rheumatologie, Berlin, Germany, ${ }^{7} \mathrm{HELIOS}$ Klinikum Berlin-Buch, Klinik f. Hämatologie, Onkologie und Tumorimmunologie, Berlin, Germany, ${ }^{8}$ Städtisches Krankenhaus St. Georg, Internistische Onkologie/ Hämatologie, Leipzig, Germany, ${ }^{9} \mathrm{HELIOS}$ Klinikum Bad Saarow, Hämatologie, Onkologie und Palliativmedizin, Bad Saarow, Germany, ${ }^{10}$ Charité - Campus Virchow-Klinikum, Klinik f. Allgemein-, Visceral- und Transplantationschirurgie, Berlin, Germany

Background: Perioperative chemotherapy with ECF or ECX is the standard approach in Europe for resectable gastro-esophageal cancer. Docetaxel significantly improves efficacy if added to cisplatin and 5-FU as palliative treatment. To translate this efficacy into a curative approach, a phase II trial combining a new regimen of docetaxel (D), cisplatin (C) with capecitabine (X) was initiated from our group.

Methods: Pts with curatively resectable gastro-oesophageal adenocarcinoma were treated with 3 pre- and 3 postoperative cycles ( 9 weeks each) of chemotherapy: D $75 \mathrm{mg} / \mathrm{m}^{2} \mathrm{~d} 1, \mathrm{C} 60 \mathrm{mg} / \mathrm{m}^{2} \mathrm{~d} 1$ and X $1875 \mathrm{mg} / \mathrm{m}^{2} / \mathrm{d} \mathrm{d} 1-14$, q3w. G-CSF was not routinely administered.

Results: Accrual is completed with now 51 pts included. Here we report on the protocol specified interim safety analysis of the preop chemo and surgery after 15 patients: male/female:15/0; med. age 65 yrs (45-73); distal esophagus/ 
Table. Toxicity of preop chemotherapy (worst grade per pt) [n;\%]

\begin{tabular}{|l|l|l|l|l|}
\hline & CTC 1 & CTC 2 & CTC 3 & CTC 4 \\
\hline diarrhoea & $3(20 \%)$ & $5(33 \%)$ & $1(7 \%)$ & $1(7 \%)$ \\
\hline nausea & $9(60 \%)$ & $3(20 \%)$ & $1(7 \%)$ & - \\
\hline asthenia & $7(47 \%)$ & $7(47 \%)$ & $1(7 \%)$ & - \\
\hline loss of appetite & $4(27 \%)$ & $4(27 \%)$ & $1(7 \%)$ & - \\
\hline neutrop. fever & - & - & $3(20 \%)$ & - \\
\hline leukopenia & $3(20 \%)$ & $4(27 \%)$ & $7(47 \%)$ & $1(7 \%)$ \\
\hline neutropenia & $2(13 \%)$ & - & $2(13 \%)$ & $11(73 \%)$ \\
\hline anemia & $4(27 \%)$ & $2(13 \%)$ & - & $1(7 \%)$ \\
\hline other & $6(40 \%)$ & $4(27 \%)$ & $\begin{array}{l}2(13 \%) \text { GI-bleed., } \\
\text { sigma-perf }\end{array}$ & - \\
\hline
\end{tabular}

GE-junction/stomach: 2/12/1 pts; ECOG PS 0/1/2: 7/8/0 pts. Dose reduction of preop chemo was necessary in $7 / 15$ pts; G-CSF was not administered. Chemo toxicity see table. Resection ypR0: $14 / 15$ pts. Surgical complications: 3/15 pts: anastomotic leakage with septic shock (1 pt), pneumonia and thrombosis (1 pt); urinary tract infection (1 pt);

Conclusions: DCX seems well tolerated and can safely be administered as preoperative chemotherapy as part of a perioperative regimen. Updated results will be presented.

Disclosure: Benedikt Gahn: No conflict of interest disclosed.

Peter Thuss-Patience: Advisory Role: Roche, Sanofi-Aventis; Honoraria: Vortragshonorar Roche, Sanofi-Aventis; Financing of Scientific Research: Studienunterstützung Roche, Sanofi-Aventis

\section{P886}

Docetaxel, Oxaliplatin and Capecitabine (TEX Regimen) in patients with advanced or metastatic Gastric or GastroEsophageal Cancer (GC/GEC). Updated results from a phase II trial of the German AIO Group

Stein, A. ${ }^{1 ;}$ Thuss-Patience, P. ${ }^{2}$; Grothe, W. ${ }^{3}$; Arnold, D. ${ }^{1}$; Wegner, F.' ; Seufferlein, T. ${ }^{1}$; Reinacher-Schick, A. ${ }^{4}$; Geissler, M. ${ }^{5}$; Hofheinz, R. ${ }^{6}$; Moehler, M.H. ${ }^{7}$; Schmoll, H.J. ${ }^{1}$

${ }^{1}$ Martin-Luther University, Halle, Germany, ${ }^{2}$ Charité, Campus VirchowKlinikum, Berlin, Germany, ${ }^{3}$ Klinikum, Fulda, Germany, ${ }^{4}$ Ruhr University, Bochum, Germany, ${ }^{5}$ Klinikum, Esslingen, Germany, ${ }^{6}$ University of Heidelberg/Mannheim, Mannheim, Germany, ${ }^{7}$ University of Mainz, Mainz, Germany

Background: Three drug regimen of docetaxel with cisplatin and 5-FU (CF) improves overall survival, response rates and quality of life in previously untreated patients with metastatic or locally advanced GC/GEC compared to CF alone (Van Cutsem et al. J Clin Oncol 2006). However, significant toxicities of the original regimen warrant the development of further modifications. Therefore, a combination of docetaxel (T), oxaliplatin (E) and capecitabine (X) was established from our group in a phase I (Grothe et al., Proc. ASCO 2006). Final data of the phase II trial are reported here.

Methods: Pts with metastatic or locally advanced GC/GEC, adequate organ function, ECOG PS 0-2, and no prior chemotherapy for advanced disease (adjuvant allowed) were enrolled. TEX regimen was administered as defined T $35 \mathrm{mg} / \mathrm{m} 2$ and $\mathrm{E} 70 \mathrm{mg} / \mathrm{m} 2$ on days (d) 1 and 8, with X $800 \mathrm{mg} / \mathrm{m} 2$ bid on d1-14 every 22 days. Toxicity assessment was done 3 -weekly while CT scans were repeated 9-weekly.

Results: 56 pts (55 evaluable) were enrolled until 02/09 (One pt was excluded due to violation of inclusion criteria). Patients characteristics: 43 male/12 female, median age 60 (29-81) years, ECOG PS 0/1/2 61\%/35\%/4\%, GC/GEC $59 \% / 41 \%$, distant metastases $94 \%$. One pt did not receive any treatment. Thus, safety population included 54 pts. A median number of 6 cycles (one cycle equals 21 days) (range 1-17) were administered. The most common toxicities (grade 3/4) were diarrhea (30\%), nausea/vomitus (13\%/9\%) and infections $(17 \%)$. Best tumor response assessment in 54 pts (RECIST criteria v1.0); Complete Response: 4\%, Partial Response: 39\% (confirmed 28\%), Stable
Disease: $39 \%$ of patients, Progressive Disease or death: $18 \%$. Disease control rate $(\mathrm{CR}+\mathrm{PR}+\mathrm{SD})$ was $82 \%$. Primary endpoint of the study, Progression Free Survival Rate after 6 months was 0.563 [90\% CI (0.439-0.669)]. Final data for PFS and overall survival data will be available $06 / 2010$ and be presented at the meeting.

Conclusion: The combination of docetaxel, oxaliplatin and capecitabine is an active and tolerable treatment for patients with advanced or metastatic GC/ GEC, with comparable efficacy to other infusional 5-FU-based triplets such as DCF and FLOT (Van Cutsem et al. J Clin Oncol 2006; Al Batran et al. Ann Oncol 2008). The TEX regimen, however, has the added benefits of oral delivery of the fluoropyrimidine component.

Disclosure: Alexander Stein: No conflict of interest disclosed. H. J. Schmoll: Advisory Role: diverse

\section{P887 \\ Inflammatory lesions of the peritoneum mimicking carcinomatosis after treatment with intravenous chemotherapy and intraperitoneal treatment with catumaxomab}

Rüssel, J. ${ }^{1}$; Stein, A. ${ }^{1}$; Behrmann, C. ${ }^{2}$; Hauptmann, S. ${ }^{3}$; Krummenerl, P. ${ }^{4}$; Jordan, K. ${ }^{1}$; Arnold, D. ${ }^{1}$

${ }^{1}$ Martin-Luther-Universität Halle-Wittenberg, Universitätsklinik für Innere Medizin IV - Onkologie/Hämatologie, Halle (Saale), Germany, ${ }^{2}$ MartinLuther-Universität Halle-Wittenberg, Universitätsklinik für Diagnostische Radiologie, Halle (Saale), Germany, ${ }^{3}$ Martin-Luther-Universität HalleWittenberg, Institut für Pathologie, Halle (Saale), Germany, ${ }^{4}$ Martin-LutherUniversität Halle-Wittenberg, Universitätsklinik für Innere Medizin I, Halle (Saale), Germany

Introduction: We report on a 54-year-old male patient diagnosed with small local lesions of the peritoneum mimicking peritoneal carcinomatosis after curative gastrectomy and postoperative adjuvant treatment with intravenous (i.v.) chemotherapy and intraperitoneal (i.p.) administration of the trifunctional antibody catumaxomab for diffuse growing gastric adenocarcinoma. Methods: The patient was initially staged as uT2 uN+ and enclosed in a prospective phase II trial which was designed to evaluate the safety and efficacy of adding postoperative i.p. catumaxomab (given on day of surgery and on postoperative days 7, 10,13 and 16) to a standard ECX chemotherapy regimen given preoperatively, followed by gastrectomy. At resection, histology did not reveal any residual malignancy (pT0 pN0). In particular, macroscopic peritoneal carcinomatosis was also excluded at this time.

Results: Suspicion of relapse with peritoneal carcinomatosis was raised at a routine follow-up CT scan 6 months later. In the area of the left upper abdomen and the surface of the right liver lobe multiple small white peritoneal lesions were evident in subsequent explorative laparoscopy hardening the tentative diagnosis. However, histological and immunohistochemical evaluation of the peritoneal plaques and fragments was consistent with definitively non-malignant inflammatory tissue whereas the histological sections demonstrated different stages of a local inflammatory process. There was no further evidence of peritoneal carcinomatosis or liver metastases at all.

Conclusions: Up to our best knowledge, this is the first case reported so far regarding development of local inflammatory lesions of the peritoneum after treatment with catumaxomab (and i.v. chemotherapy). We discuss potential pathogenetic mechanisms, such as the release of pro-inflammatory and cytotoxic cytokines initiated by the binding of catumaxomab to peritoneal cells. The development of local inflammatory lesions of the peritoneum should be considered in the differential diagnosis with gastrointestinal epithelial malignancies after i.v. chemotherapy or i.p. treatment with catumaxomab.

Disclosure: No conflict of interest disclosed. 
P888

\section{Disseminated intravasal coagulation associated with relapse of gastric cancer as bone marrow carcinomatosis effectively treated with Oxaliplatin and Fluorouracil}

\section{Kim, J.-W. ${ }^{1}$; Hanus, L. ${ }^{1}$; Fischer, T. ${ }^{1}$; Hütten, H. $^{1}$ \\ ${ }^{1}$ Universitätsklinikum Magdeburg, Hämatologie und Onkologie, Magdeburg, Germany}

Introduction: Acute disseminated intravasal coagulation (DIC) presenting with a fibrinolytic phenotype is a rare but life-threatening complication in advanced gastric cancer. Treatment of the underlying malignancy is a prerequisite to resolve the severe coagulopathy. Due to the limited data available a standard therapy regimen is not yet established. However, 5-fluorouracil (5-FU) is highly active in gastric and colorectal cancers and is favoured by some authors for its negligible myelosuppressive properties for treatment of cancer-related DIC. Here, we applied a combination of 5-FU with oxaliplatin as liver dysfunction argued against the use of taxans or irinotecan.

Case: On admission a 42 year old female presented with persisting gingival hemorrhage and complained of malaise and easy bruisability. She had a history of stage 3 diffuse-type gastric cancer and underwent subtotal gastrectomy three years earlier followed by an adjuvant chemotherapy with 5- FU and cisplatin. Laboratory findings confirmed overt DIC according to the ISTH scoring system with severe hypofibrinogenamia and decreased factor XII activity. Markedly elevated liver enzymes suggested liver dysfunction due to DIC. Whereas endoscopy showed no signs of local tumor relapse, CT-scan revealed multiple bone lesions. Finally, bone marrow examination revealed relapse of gastric cancer with diffuse bone marrow infiltration. The clinica picture was dominated by hyperfibrinolysis with severe bleeding. Tranexamic acid was administered followed by chemotherapy consisting of oxaliplatin $\left(85 \mathrm{mg} / \mathrm{m}^{2}\right)$ on day one and 5 -fluorouracil $\left(400 \mathrm{mg} / \mathrm{m}^{2}\right.$ bolus and $600 \mathrm{mg} / \mathrm{m}^{2} 22 \mathrm{~h}$ infusion) on days one and two. DIC parameters and liver enzymes normalized within three days after starting chemotherapy. Signs of bleeding also improved rapidly.

Conclusion: Oxaliplatin and 5-FU in combination with tranexamic acid proved highly effective in resolving paraneoplastic DIC presenting with fibrin olytic phenotype in a patient with bone marrow relapse from gastric cancer. As DIC and tumor infiltration often give rise to various patterns of organ dysfunction, chemotherapy needs to be tailored to the specific situation found in the patient.

Disclosure: No conflict of interest disclosed.

\section{P889}

Signet ring cell carcinoma of the stomach is significantly associated with poor prognosis and diffuse gastric cancer (Lauren's) - single center experience of 160 cases

Hass, H.G. ${ }^{1}$; Smith, U. ${ }^{2}$; Jäger, C. ${ }^{3}$; Schäffer, M. ${ }^{3}$; Wellhäußer, U. ; Markmann, H.-U.5; Hehr, T.6; Nehls, O.7; Denzlinger, C. ${ }^{8}$ ${ }^{1}$ Paracelsus-Klinik Scheidegg, Scheidegg, Germany, ${ }^{2}$ Onkologischer Schwerpunkt Stuttgart (OSP), Stuttgart, Germany, ${ }^{3}$ Marienhospital Stuttgart, Abdominal-, Viszeral und Thoraxchirurgie, Stuttgart, Germany, ${ }^{4}$ Marienhospital Stuttgart, Gastroenterologie, Hepatologie und Pulmologie, Stuttgart, Germany, ${ }^{5}$ Marienhospital Stuttgart, Pathologie, Stuttgart, Germany, ${ }^{6}$ Marienhospital Stuttgart, Radioonkologie, Stuttgart, Germany, ${ }^{7}$ Hospital Bad Cannstatt, Onkologie, Gastroenterologie und Hepatologie, Stuttgart, Germany, ${ }^{8}$ Marienhospital Stuttgart, Onkologie, Hämatologie und Palliativmedizin, Stuttgart, Germany

Background: To evaluate survival rates and treatment response in stage I-IV gastric cancers in relation to tumor stage (TNM), histology, Lauren's classification and tumor localisation.

Patients/methods: Clinical and histopathological data of 160 patients with stage I-IV gastric cancer were analyzed in this retrospective study.

Results: Most patients $(73.1 \%)$ showed an advanced or metastatic tumor stage

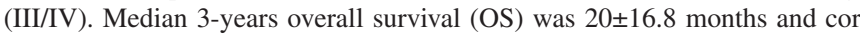
related significantly with tumor stage (I: OS $30.6 \pm 15$ months vs. IV: $10.4 \pm 9.3$ months; $\mathrm{P}<0.0001)$. III/IV tumors were significantly more often poorly differentiated $(\mathrm{G} 3 ; \mathrm{P}=0.011)$ and localisated in the corpus region. In most of these tumors signet ring cell cancers (SRC) were found when compared with locally limited gastric cancers $(43.1 \%$ vs. $16.3 \% ; \mathrm{P}=0.002)$. In $\mathrm{SRC}$ tumors significantly more often the diffuse subtype after Lauren $(7.5 \%$ vs. $63.2 \% ; \mathrm{P}<$ 0.0001 ) or poor differentiation (G3 in $95 \%$ vs. $73 \%, \mathrm{P}=0.001)$ were found and occurred predominantly in female and younger patients.

Conclusions: SRC gastric cancer is correlated with poor histopathological criteria and poor prognosis when compared with other histological subtypes. These observations underline the need for more effective treatment in addition to standard approaches such as conventional neoadjuvant/perioperative chemotherapy and radical D2 dissection.

Disclosure: No conflict of interest disclosed.

P890

Ion transport proteins control pancreatic ductal adenocarcinoma

Schwab, A. ${ }^{1}$; Stock, C. ${ }^{1}$; Swietach, P. ${ }^{2}$; Hulikova, A. ${ }^{2}$; Vaughan Jones, R. ${ }^{2}$; Pedersen, S.F.'; Andersen, A.-D. ${ }^{3}$; Hoffmann, E.'; Novak, I. ${ }^{3}$; Reshkin, S. ${ }^{4}$ Prevarskaya, N. ${ }^{5}$ : Lemonnier, L.5. Borowiec, A.-S. ${ }^{5}$; Arcangeli, A. ; Zanieri, F. ${ }^{6}$; D'Amico, M. ${ }^{6}$; Fraser, S.P. ${ }^{7}$; Djamgoz, M.B. ${ }^{7}$; Alves, F. ${ }^{8}$ Kalthoff, H. $^{9}$; Pardo, L. ${ }^{10}$; Stühmer, W. ${ }^{10}$

${ }^{1}$ Institute of Physiology II, Münster, Germany, ${ }^{2}$ Department of Physiology, Anatomy and Genetics, Oxford, United Kingdom, ${ }^{3}$ Department of Biology, Copenhagen, Denmark, ${ }^{4}$ Department of General and Environmental Physiology, Bari, Italy, ${ }^{5}$ Laboratory of Cell Physiology, INSERM U 1003 Lille, France, ${ }^{6}$ Department of Pathology and Experimental Oncology, Florence, Italy, ${ }^{7}$ Imperial College, London, United Kingdom, ${ }^{8}$ Department of Hematology and Oncology, University Medical Center, Göttingen, Germany, ${ }^{9}$ Division of Molecular Oncology, Kiel, Germany, ${ }^{10}$ Max-PlanckInstitute of Experimental Medicine, Göttingen, Germany

Introduction: Pancreatic ductal adenocarcinoma (PDAC) has a very poor prognosis. There has been no improvement in the clinical outcome for several decades. Mature ductal epithelial cells from normal pancreas are unique because they express an array of carbonic anhydrases (CAs), ion channels and transporters that allow for copious secretion of $\mathrm{HCO}^{-}$. This could have major implications for $\mathrm{pH}_{\mathrm{i}}$ and $\mathrm{pH}_{\mathrm{e}}$ regulation and thereby impact on tumour progression since membrane proteins involved in ion transport control many "hallmarks of cancer". So far, there is little information available on the role of these proteins in PDAC. "Data mining" of published gene arrays of PDAC tissue reveals distinct differences when compared to arrays from non-cancerous pancreatic tissues. We describe experiments to elucidate the role of these membrane proteins in PDAC progression.

Methods: We used several PDAC cell lines, primary human PDAC samples as well as orthotopic implants of human pancreatic cell lines into immunodeficient mice. Expression and function of transport proteins was assessed by PCR, Western blot, immunohistochemistry, ionic imaging, patch clamp techniques and video microscopy.

Results: PDAC cells express many proteins involved in ion transport that had been linked to tumour progression, e.g. pH-regulatory proteins NHE1, NBCn1, CA IX, $\mathrm{K}^{+}$channels $\mathrm{K}_{\mathrm{Ca}} 3.1$, EAG, hERG, voltage-gated $\mathrm{Na}^{+}$channels and store-operated $\mathrm{Ca}^{2+}$ channel components. NHE1 and CA IX are upregulated by hypoxia-inducible factor. Extensive CA IX staining in sections of mouse duodenum and stomach showed high metastatic potential of pancreatic cancer cell lines. NHE1 and $\mathrm{K}_{\mathrm{C}} 3.1$ blockade impair PDAC cell migration on defined extracellular matrices. Digestion of the extracellular matrix is attenuated by NHE1 inhibition.

Conclusions: The experiments show that PDAC cells functionally express a number of transport proteins whose pathophysiological importance in tumour progression is well established. Hence, these membrane proteins could constitute a new class of therapeutic targets for PDAC.

Disclosure: No conflict of interest disclosed. 
P891

\section{Safety and Efficacy of Sorafenib (Sor) in Patients (Pts) with Advanced Hepatocellular Carcinoma (HCC): Subgroup Analyses of the SHARP Trial by Baseline (BL) Transaminase (ALT/AST)/a-Fetoprotein (AFP) and Bilirubin (Bil) Levels}

Dollinger, M.M. ${ }^{1}$; Raoul, J.L. ${ }^{2}$; Sherman, M. ${ }^{3}$; Nadel, A. ${ }^{4}$; Lentini, G. ${ }^{5}$; Moscovici, M. ${ }^{6}$; Voliotis, D. ${ }^{4}$; Meinhardt, G. ${ }^{4}$; Bruix, J.7; Llovet, J.M. ${ }^{7,8}$

${ }^{1}$ Klinik und Poliklinik für Innere Medizin I, Martin-Luther-Universität, Halle/ Saale, Germany, ${ }^{2}$ Centre Eugène Marquis, Rennes, France, ${ }^{3}$ Toronto General Hospital, Toronto, Canada, ${ }^{4}$ Bayer HealthCare Pharmaceuticals, Montville, United States, ${ }^{5}$ Bayer Vital $\mathrm{GmbH}$, Leverkusen, Germany, ${ }^{6}$ Bayer Schering Pharma, Milan, Italy, ${ }^{7}$ Barcelona Clinic Liver Cancer (BCLC) Group, Liver Unit, CIBERehd, IDIBAPS, Hospital Clinic, Barcelona, Spain, ${ }^{8}$ Mount Sinai Liver Cancer Program, Mount Sinai School of Medicine, New York, United States

Background: Results of the phase 3, double-blind, placebo (Pla)-controlled SHARP trial demonstrated that Sor is effective and safe for the treatment of advanced HCC. As hepatic function influences treatment options, we examined the effect of Sor on hepatic function, as indicated by bil levels, and performed subset analyses of SHARP according to BL levels of ALT/AST/AFP. Methods: Pts $(\mathrm{N}=602)$ were randomized 1:1 to receive Sor $400 \mathrm{mg}$ bid or Pla in 6-week cycles. Endpoints included overall survival (OS), disease-control rate (DCR; defined as complete/partial response or stable disease by RECIST maintained for $\geq 28$ days from first demonstration of response), time to progression (TTP), and safety. Pts were grouped by BL levels of ALT/AST (normal, mild, or moderate) and AFP (normal or abnormal). Bil was measured at BL and day 1 of each cycle.

Results: TTP, OS, and DCR are shown below. No notable differences in the safety profiles were observed between pts with normal vs elevated ALT/AST/ AFP levels. Mean BL levels of bil in the Sor and Pla groups were 0.83 and $0.88 \mathrm{mg} / \mathrm{dL}$, respectively. A transient elevation in bil was observed in Sor pts at cycle 2. Mean change in bil at last cycle in the Sor and Pla groups were 1.01 and $0.95 \mathrm{mg} / \mathrm{dL}$, respectively.

Conclusions: These results suggest that Sor is an effective treatment for $\mathrm{HCC}$, irrespective of ALT/AST/AFP levels, and that hepatic function (bil levels) is not appreciably affected by Sor treatment.

Disclosure: M Dollinger: Advisory Role: Bayer, Novartis; Financing of Scientific Research: Bayer, Novartis, Roche, BMS

J Llovet: Advisory Role: Bayer; Honoraria: Bayer; Financing of Scientific Research: Bayer

\section{P892}

Mini-laparoscopy as diagnostic instrument in patients with gastrointestinal malignancies

Krause, F. '; Damm, M.2; Ulmer, M. ${ }^{2}$; Wehrmann, K. ${ }^{2}$; Kayser, T.2; Schiedeck, T.; Caca, K. ${ }^{2}$; Schwella, N. ${ }^{2}$

${ }^{1}$ Klinikum Ludwigsburg, Neurologie, Ludwigsburg, Germany, ${ }^{2} \mathrm{Klinikum}$ Ludwigsburg, Gastroenterologie/Onkologie, Ludwigsburg, Germany,

${ }^{3}$ Klinikum Ludwigsburg, Allgemeinchirurgie, Ludwigsburg, Germany

Introduction: Peritoneal carcinosis and/or small metastases of the liver are not sufficiently recognized with conventional radiological imaging technology. In this work a retrospective analysis examines the results of mini-laparoscopy as a staging procedure in patients with gastrointestinal malignancies. Aim of the mini-laparoscopy is the exclusion or verification of peritoneal carcinosis and/or liver metastases before potential surgery.

Patients/methods: Between Nov 2005 and Dec 2008 mini-laparoscopy was carried out in 132 patients suffering from the following gastrointestinal malignancies: gastric ca. $(n=44)$, pancreatic ca. $(n=43)$, intrahepatic or extrahepatic bile duct ca. $(n=14)$, carcinoma of the papilla vateri $(n=11)$, gallbladder ca. $(n=6)$, adenocarcinoma of the esophagus $(n=8)$ and other miscellaneous malignant tumors $(n=6)$. Diagnostic findings of the mini-laparoscopy were rated as positive if peritoneal carcinosis and/or liver metastases could be macroscopically detected and verified by histological methods. Overall, 93 patients $(70,4 \%)$ underwent surgery with curative intention $(n=70)$ or within a palliative setting $(n=23)$. Other 34 patients did not have surgery and 5 patients continued treatment in other hospitals and were lost for follow-up.

Results: The mini-laparoscopy was macroscopically suspect with histological evidence of carcinoma in 17 of 132 cases $(12,9 \%)$. In 11 of 132 patients $(8,3 \%)$ the suspicious macroscopic findings of the mini-laparoscopy could not be confirmed by histological methods. In 104 of 132 patients $(78,8 \%)$ the findings of the mini-laparoscopy were macroscopically negative. In the 17 cases with histologically proven peritoneal carcinosis and/or liver metastases 15 patients did not undergo surgery. Out of 115 cases with macroscopically negative $(n=104)$ or macroscopically suspect, but histologically negative laparoscopical findings $(n=11) 91$ patients underwent surgery. In only 16 of 91 cases $(17,6 \%)$ liver metastases $(n=7)$, peritoneal carcinosis $(n=7)$ or peritoneal carcinosis plus liver metastases $(n=2)$ were found during surgery. Thus, the macroscopically or histologically negative results of the mini-laparoscopy could be confirmed in 75 patients at surgery $(82,4 \%)$.

Conclusion: Staging mini-laparoscopy is able to detect occult peritoneal carcinosis and/or liver metastases in a significant number of patients with gastrointestinal malignancies. These patients should be prevented from an unnecessary and straining surgical treatment.

Disclosure: No conflict of interest disclosed.

Table 1. (for Abstract P891)

\begin{tabular}{|c|c|c|c|c|c|c|c|}
\hline \multirow[t]{2}{*}{ BL Population } & \multicolumn{2}{|l|}{$\mathrm{n}$} & \multicolumn{2}{|l|}{ TTP (Sor/Pla) } & \multicolumn{2}{|l|}{ OS (Sor/Pla) } & \multirow{2}{*}{$\begin{array}{l}\operatorname{DCR}(\%) \\
\text { (Sor/Pla) }\end{array}$} \\
\hline & Sor & Pla & Median (mo) & HR $(95 \%$ CI) & Median (mo) & HR $(95 \%$ CI $)$ & \\
\hline Total & 299 & 303 & $5.5 / 2.8$ & $0.58(0.45,0.74)$ & $10.7 / 7.9$ & $0.69(0.55,0 ; 87)$ & $43 / 32$ \\
\hline $\begin{array}{l}\text { Normal ALT/AST } \\
(<1.8 \times \mathrm{ULN})\end{array}$ & 152 & 153 & $5.7 / 3.9$ & $0.57(0.41,0.80)$ & $11.6 / 8.8$ & $0.68(0.49,0.93)$ & $53 / 39$ \\
\hline $\begin{array}{l}\text { Moderately elevated } \\
\text { ALT/AST }(>3.0 \times \\
\text { ULN })\end{array}$ & 68 & 72 & $5.8 / 2.6$ & $0.54(0.31,0.94)$ & $6.3 / 4.6$ & $0.71(0.46,1.09)$ & $32 / 21$ \\
\hline $\begin{array}{l}\text { Normal AFP }(\leq 40 \\
\mu \mathrm{g} / \mathrm{L})\end{array}$ & 111 & 97 & $9.6 / 4.1$ & $0.72(0.46,1.11)$ & $12.4 / 9.5$ & $0.76(0.51,1.13)$ & $48 / 41$ \\
\hline $\begin{array}{l}\text { Elevated AFP (>40 } \\
\mu \mathrm{g} / \mathrm{L})\end{array}$ & 171 & 194 & $5.3 / 2.7$ & $0.55(0.40,0.75)$ & $9.4 / 7.0$ & $0.72(0.55,0.95)$ & $40 / 26$ \\
\hline
\end{tabular}


P893

\section{Hepatic arterial infusion chemotherapy (HAI) - a feasible therapeutic option for liver tumours?}

Sinn, M. ${ }^{1}$; Nicolaou, A. ${ }^{1}$; Podrabsky, P. ${ }^{2}$; Gebauer, B. ${ }^{2}$; Hildebrandt, B. ${ }^{1}$; Dörken, B. ${ }^{1}$; Riess, $H^{1}{ }^{1}$

${ }^{1}$ Charité - Universitätsmedizin Berlin, Klinik für Hämatologie/Onkologie, Berlin, Germany, ${ }^{2}$ Charité - Universitätsmedizin Berlin, Klinik für

Radiologie, Berlin, Germany

Introduction: Chemotherapy with Gemcitabine/Cisplatin (phase III tria NEJM 2010) for unresectable cholangiocarcinoma (CCC) and the accreditation of Sorafenib in the first line therapy of hepatocellular carcinoma (HCC improved the very limited therapeutic options of these patients (pts). HAI may be an additional chance in the treatment strategies of liver tumours.

Methods: In pts with unresectable histologically confirmed advanced CCC or HCC without relevant distant metastases catheters were placed interventionally via a. femoralis sup. in a. hepatica or a. lienalis and connected to a port chamber. HAI of 5 -FU $600 \mathrm{mg} / \mathrm{mq} / 2 \mathrm{~h}$ combined with Folinic Acid $170 \mathrm{mg} /$ $\mathrm{mq} / 2 \mathrm{~h}$ (FF) followed by Oxaliplatin $85 \mathrm{mg} / \mathrm{mq} / 2 \mathrm{~h}$ were given every 2 weeks. Pts were followed prospectively with regard to port complications, response rate, TTP and OS

Results: Between 2003 and end of december 200974 Pts (37 CCC/37 HCC) obtained port implantation in local anasthesia without complications and were treated with HAI chemotherapy. Median age was 60,5 years (range 38-80), median treatment duration 3.9 months $(0.6-41.7) .18$ pts developed manageable port complications ( 6 thrombosis, 6 dysfunction, 4 infection, 1 malposi tion, 1 arterial spasm). For CCC III ${ }^{\circ}$ toxicity occured in 9 pts (1 diarrhea, 1 PNP, 1 nausea, 2 leukopenia, 4 thrombopenia). End of treatment (48 pts) was due to tumour progression in 35 Pts, to port complications in 4 Pts. Median TTP was 5.9 months (0.6- 41.7). Median OS was 10.0 months, (0.9-69.0+), 1year-survival $40 \%$

Conclusions: Prolonged HAI chemotherapy with Oxaliplatin, 5-FU/FA is feasible and well tolerated.

$\mathrm{HAI}$ is an promising therapeutic option in the enlarging treatment possibilities of $\mathrm{CCC}$ and $\mathrm{HCC}$

Disclosure: Marianne Sinn: No conflict of interest disclosed.

Hanno Riess: Honoraria: Amgen, Bayer, Boehringer, GSK, Lilly, Merck, Novartis, Roche, Sanofi Aventis

P894

\section{Clinical experience in use of catumaxomab - a single center solution of problems}

\section{Steinborn, W. ${ }^{1}$; Stein, W. ${ }^{1}$; Kokowski, K. ${ }^{1}$; Kiehl, M.G. ${ }^{1}$}

${ }^{1}$ Klinikum Frankfurt (Oder) $\mathrm{GmbH}$, Klinik für Innere Medizin I, Frankfurt (Oder), Germany

Introduction: Malignant ascites is associated with poor prognosis an reduced quality of life as a typical late-stage manifestation of epithelial cancer. Treatment with the trifunctional antibody catumaxomab resulted in a clinically relevant prolongation of puncture-free survival. One of the major problems associated with catumaxomab are cytokine-release-related symptoms - fever, nausea, abdominal pain, and vomiting.

Methods: Patients with epithelial cell adhesion molecule (EpCAM)- positive malignant ascites were observed concerning occurrence of adverse events during receiving the four doses of catumaxomab. Two groups were built, which obtained a different prophylaxis of potential side effects.

Results: Among eight patients, four in each group, cytokine-release-related symptoms (CRRS) are occurred in all patients. The first group, which got the standard prophylaxis (paracetamol before start of infusion), CRRSs were limited to the duration of catumaxomab treatment with onset of a couple of hours after catumaxomab administration and a median duration of 2 days. The other group received a second administration of paracetamol at hour 4 during infusion and additional ondansetron before and 4 hours after start of infusion. The side events were very rare and the infusion were better tolerated than in the first group. Most of these symptoms were mild to moderate.

Conclusions: CRRSs are a common occurrence due to its mode of activating $\mathrm{T}$ cells and accessory cells. The management of possible side effects with intensified prophylaxis tends to e better tolerance of the treatment without influence of the clinical effect.

Disclosure: No conflict of interest disclosed

\section{Posterdiskussion Immuntherapie \\ P895 \\ Human Langerhans cells reconstitute in skin xenografts}

Hemmerling, J. ${ }^{1}$; Wegner-Kops, J.2; Sommer, M.'; Wagner, E.M. ${ }^{1}$; Hartwig, U.F. ${ }^{1}$; Schopf, R.E. ${ }^{2}$; Theobald, M. ${ }^{1} ;$ Herr, W. ${ }^{1}$;

Meyer, R.G. ${ }^{1}$

${ }^{1}$ Universitätsmedizin der Johannes Gutenberg-Universität Mainz, III. Medizinische Klinik, Mainz, Germany, ${ }^{2}$ Universitätsmedizin der Johannes Gutenberg-Universität Mainz, Dermatology, Mainz, Germany

Dendritic cells (DC) of the skin, e.g. epidermal Langerhans cells (LC), are potent regulators of $\mathrm{T}$ cells. They therefore are interesting targets for autologous T-cell stimulation in the context of vaccine-strategies as well as for the manipulation of allo-reactive T-cells in graft-versus-host disease (GVHD). In an attempt to study the biology of human LC in vivo, we transplanted human skin to immunodeficient NOD/SCID/gc $\mathrm{c}^{\text {null }}$ (NSG) mice and studied the impact of xeno-transplantation on the distribution of LC. We analyzed skin xenografts at different time points after transplantation and found that during wound healing, LC were absent in grafts prepared 4 to 6 weeks after transplantation. However, in many animals analyzed beyond week 6, LC were again present with almost normal distribution. These findings were re-confirmed by staining with antibodies against human CD1a, CD207/Langerin, S100 and HLA-DR. CD11c positive human dermal cells were also transiently reduced in numbers, but never vanished from the dermis after xenografting. In subsequent experiments, we analyzed the skin grafts by sequential biopsies and again demonstrated that more than $50 \%$ of the skin grafts were devoid of LC in week 6. Three to 5 weeks later, LC were detected again in these transplants with a slightly reduced density compared to normal skin. In congenic mouse models of hematopoietic stem cell transplantation, murine LC have been shown to re-populate after local skin injury without the need of blood-derived precursors. Up to now, there are only few data supporting this hypothesis for human LC. By demonstrating the re-establishment of LC in the xenografts in the absence of any human hematopoiesis, our data confirm that human LC are able to re-populate the skin after local depletion. We further analyzed the proliferative activity of LC in the xenografts by double staining of CD207/ Langerin-positive cells with Ki67 and found a significantly increased proliferative activity of LC compared to healthy skin (31\% vs. $5 \%)$. Proliferation is a unique feature of LC among DC. Our data suggest that this contributes to LC-reconstitution. In summary, we introduce a human skin xenograft-model that allows studying the biology of LC and dermal DC. Our findings help to unravel the phenomenon of local LC-reconstitution in human skin. This model might also help to study the role of skin DC for inflammatory skin disease and skin GVHD as well as for vaccine-strategies in vivo.

Disclosure: No conflict of interest disclosed.

\section{P896}

\section{Functional deficiency of chimeric antigen receptors expressing $T$ cells is due to reduced phosphorylation of signal proteins in key signal pathways upon stimulation}

\section{Duell, J. ${ }^{1}$; Lurati, S. ${ }^{1}$; Bedke, T. ${ }^{1}$; Einsele, H. ${ }^{1}$; Pule, M. ${ }^{2}$,} Rossig, C. ${ }^{3}$; Topp, M.S. ${ }^{1}$

${ }^{1}$ Medizinische Klinik und Poliklinik II, Würzburg, Germany, ${ }^{2}$ Department of Haematology, UCL, London, United Kingdom, ${ }^{3}$ Department of Pediatric Hematology and Oncology, Münster, Germany

Introduction: A promising strategy for tumor therapy is the adoptive transfer of tumor specific T cells which are endowed with chimeric antigen receptors (CAR). First generation CARs are constructed by by linking single chain antibodies to the signaling domain of the $\mathrm{CD} 3 \zeta$ chain. However, clinical trials were disappointing as adoptive transferred T-cells showed only limited clinical activity. We therefore hypothesized that CAR expressing T-cells in comparison 
to unmodified T-cells display signaling defects when stimulated via their CARs.

Methods: CMV-specific CD8 ${ }^{+}$T-cells were generated and modified with first generation CAR targeting CD19. T-cell receptor (TCR) and CAR expression were quantified by Quantibright beads. Effector functions of both T-cell populations were analyzed for specific lysis, cytokine production (IFN-g, TNF-a) and proliferation (CSFE) as well as phosphorylation of ERK, PLC- $\gamma$, p38 and JNK upon target cell stimulation.

Results: CMV-specific TCRs and CARs are expressed at same density levels and T-cells show equal lysis of target cells. In contrast, cytokine production and proliferation was reduced in CAR expressing T-cells when compared to CMV-specific CD8 ${ }^{+}$T-cells upon target exposure. PLC- $\gamma$ was phosphorylated within minutes after target contact by CMV-specific TCRs whereas CAR transduced T-cells showed no significant phosphorylation of PLC- $\gamma$ to target cell exposure. CMV-specific stimulation induced maximum phosphorylation of the MAP-kinases ERK, JNK and p38 after 20, 45 and 120 min, respectively. In T-cells activated via CARs, maximum phosphorylation was reduced to $66 \%$ for ERK, $23 \%$ for JNK and $40 \%$ for p38 in comparison to CMV-TCRs. Furthermore, phosphorylation in CAR transduced T-cells returned to base line 20-45\% earlier than in T-cells stimulated via their TCR.

Conclusion: CAR expressing T-cells show functional signs of split anergy, with efficient target elimination but failure to produce significant levels of cytokines and to proliferate in response to target stimulation. Split anergy is not due to reduced expression of the CARs but to a complete lack of phosphorylation of PLC- $\gamma$ as well as reduced phosphorylation of MAP-kinases ERK, p38 and JNK. These results potentially explain why CAR expressing T-cells fail to show significant clinical efficacy. Analysis of adequate phosphorylation, as proposed here, may be a powerful tool to identify the most promising second generation CARs for clinical studies.

Disclosure: No conflict of interest disclosed.

\section{P897}

\section{Dasatinib modulates NK cell cytotoxicity by interfering with early activation events}

Kempf, K. ${ }^{1}$; Urlaub, D. ${ }^{2}$; Watzl, C. ${ }^{2}$; Einsele, H. ${ }^{1}$; Seggewiss, R. ${ }^{1}$ ${ }^{1}$ Department of Internal Medicine II, University of Würzburg, Hematology/ Oncology, Würzburg, Germany, ${ }^{2}$ Institute of Immunology, University of Heidelberg, Heidelberg, Germany

Introduction: As natural killer (NK) cell immunotherapy against lymphoma is still not as successful as hoped for, pharmacological approaches seem warranted to enhance NK cell activity. For the SRC/BCR-ABL inhibitor dasatinib an inhibition of NK cell cytotoxicity has been demonstrated (Blake et al. 2008). However, after $24 \mathrm{~h}$ pretreatment of NK cells we found an enhancement of cytokine secretion and cytotoxic activity against selected lymphoma cell lines by dasatinib (Hassold et al. 2009). Therefore, we analysed the mechanisms of action by focussing on early events of NK cell activation and the role of specific receptor-ligand systems at clinical relevant dasatinib doses of 10 and 50nM.

Methods: Peripheral blood mononuclear cells or expanded polyclonal NK cells $\left(\mathrm{CD} 3^{-} \mathrm{CD} 56^{+}\right)$were used for in vitro assays. For expansion we co-cultured non-adherent peripheral blood lymphocytes of healthy human donors with irradiated RPMI 8866 feeder cells for 8-10 days. Functional outcomes assessed included NK cell conjugate formation over time $(0,20,60 \mathrm{~min})$ with lymphoma cells (K562, Daudi) and intracellular $\mathrm{Ca}^{2+}$ flux after costimulation of activating NK cell receptors (2B4, NKG2D) by antibody-mediated crosslinking. As the CD94/NKG2A receptor system regulates inhibitory signal transduction we evaluated its expression after $24 \mathrm{~h}$ treatment of freshly isolated PBMCs with dasatinib.

Results: Conjugate formation between NK and target cells was dose dependently inhibited when dasatinib was present in the assay $(n=4)$. In contrast, no inhibition was observed when NK cells were pretreated for $24 \mathrm{~h}$ with dasatinib, the drug washed off and the assay performed $(n=4)$. Dasatinib inhibited NK cell $\mathrm{Ca}^{2+}$ flux completely at 50nM and partially with a delayed flux at $10 \mathrm{nM}$ $(n=3)$. Regarding CD94/NKG2A expression a significant upregulation with dasatinib $10 \mathrm{nM}$ at $24 \mathrm{~h}$ compared to $0 \min (\mathrm{n}=5, \mathrm{p}<0.05)$ and to untreated control $(\mathrm{p}<0.01)$ was observed in the cytotoxic $\mathrm{CD} 94^{\text {low }} \mathrm{CD} 56^{\mathrm{dim}}$ subset; no significant effects was seen in the functional intermediary (CD94 ${ }^{\text {high }} \mathrm{CD} 56^{\mathrm{dim}}$ ) and cytokine secreting (CD94 ${ }^{\text {high }} \mathrm{CD} 56^{\text {bright }}$ ) subset.
Conclusions: Dasatinib interferes with early SRC kinase dependent NK cell activation events like conjugate formation and PLC $\gamma$-mediated $\mathrm{Ca}^{2+}$ flux in a reversible manner leading to inhibition of NK cell effector functions. The increased frequency of the cytotoxic CD $94^{\text {low }} \mathrm{CD} 56^{\mathrm{dim}}$ subset may be a mechanism of cytotoxicity enhancement after $24 \mathrm{~h}$ pretreatment with dasatinib useable in the clinic.

Disclosure: Kristina Kempf: No conflict of interest disclosed.

Ruth Seggewiss: Honoraria: Vortragstätigkeit für Bristol-Myers-Squibb

\section{P898 \\ Hyperactivable NFAT1 ameliorates autoimmune encephalitis in vivo}

Ghosh, S. ${ }^{1}$; Koralov, S. B. ${ }^{1}$; Stevanovic, I. ${ }^{1}$; Sundrud, M.S. ${ }^{1}$; Sasaki, Y. ${ }^{2}$; Rajewsky, K. ${ }^{1}$; Rao, A. ${ }^{1}$; Müller, M.R. ${ }^{1,3}$

${ }^{1}$ Harvard Medical School, Dept. of Pathology and Immune Disease Institute, Boston, United States, ${ }^{2}$ RIKEN Center for Developmental Biology, Laboratory of Stem Cell Biology, Kobe, Japan, ${ }^{3}$ Universitätsklinikum

Tübingen, Abt. für Hämatologie, Onkologie, Immunologie, Rheumatologie und Pulmonologie, Tübingen, Germany

Introduction: $\mathrm{CD} 4 \mathrm{~T}$ cells differentiate into diverse subsets to coordinate the adaptive immune response. $\mathrm{T}_{\mathrm{H}} 1$ and $\mathrm{T}_{\mathrm{H}} 2$ effector subsets produce IFN- $\gamma$ and IL-4, while $T_{H} 17$ cells are regulators of autoimmune inflammation and characteristically produce IL-17 and IL-22. Naive T cells can also differentiate into induced $\mathrm{T}$ regulatory $\left(\mathrm{iT}_{\mathrm{reg}}\right.$ ) cells. NFAT proteins are highly phosphorylated and reside in the cytoplasm of resting cells. Upon dephosphorylation by calcineurin, NFAT proteins translocate to the nucleus, where they orchestrate developmental and activation programs in diverse cell types. Here, we investigated the role of the Ca/NFAT signaling pathway in $\mathrm{T}$ cell lineage differentiation and the development of autoimmune diseases.

Methods: We generated transgenic mice conditionally expressing a hyperactivable version of NFAT1 (AV-NFAT1). To restrict AV-NFAT1 expression to the $\mathrm{T}$ cell compartment, AV-NFAT1 transgenic mice were bred to CD4-Cre transgenic mice.

Results: While the numbers of CD4+CD25+ "natural" $\mathrm{T}_{\text {reg }}$ cells in peripheral lymphoid organs and their suppressive functions were slightly decreased in AV mice, $\mathrm{iT}_{\text {reg }}$ generation from CD4+CD25- $\mathrm{T}$ cells of AV mice was markedly enhanced. $\mathrm{T}_{\mathrm{H}} 17$ cells generated in vitro from CD4 T cells of AV mice exhibited dramatically increased expression of both IL-10 and IL-17. To investigate putative NFAT binding sites in the IL-10 and IL-17 gene loci, we performed chromatin immunoprecipitation experiments. We show that NFAT1 can bind at the IL-17 locus at 3 out of 9 CNS regions which are accessible specifically during $\mathrm{T}_{\mathrm{H}} 17$ but not during $\mathrm{T}_{\mathrm{H}} 1$ and $\mathrm{T}_{\mathrm{H}} 2$ differentiation. Furthermore, we provide evidence that NFAT1 binds one CNS region in the IL10-locus in $\mathrm{T}_{\mathrm{H}} 17$ cells. To verify our observations in vivo, we induced experimental autoimmune encephalitis (EAE). While wild type animals showed a normal course of disease, AV mice showed a markedly weaker disease phenotype with less severe degrees of paralysis and accelerated kinetics of remission. Moreover, at the peak of the response, there were fewer CD4+CD25- but more CD4+CD25+ $\mathrm{T}$ cells in the CNS of AV animals. Surprisingly, these cells produced significantly more IL-2, IL-17 and IFN- $\gamma$ upon restimulation, even though they displayed decreased disease.

Conclusions: Our data provide strong evidence that NFAT1 contributes to the regulation of IL-10 and IL-17 expression in $\mathrm{T}_{\mathrm{H}} 17$ cells and show that increasing NFAT1 activity can ameliorate autoimmune encephalitis.

Disclosure: No conflict of interest disclosed. 
P899

Isolation and characterization of MHC class II-restricted T cells with specificity for the tumor-associated antigen FMNL1

Weigand, L.U. ${ }^{1}$; Liang, X. ${ }^{1}$; Schuster, I. ${ }^{2}$; Eppinger, E. ${ }^{1}$; Han, Y.1; Schiemann, M. ${ }^{3}$; Kremmer, E. ${ }^{2}$ Moosmann, A. ${ }^{4}$; Peschel, C. ${ }^{1}$; Krackhardt, $A .^{1}$

${ }^{1}$ Technische Universität München/ Klinikum Rechts der Isar, 3. Med. Klinik Hämatologie/Onkologie, München, Germany, ${ }^{2}$ Helmholtz Zentrum München, Institut für molekulare Immunologie, München, Germany, ${ }^{3}$ Technische Universität München/ Klinikum Rechts der Isar, Institut für medizinische Mikrobiologie, Immunologie und Hygiene, München, Germany, ${ }^{4} \mathrm{Helmholtz}$ Zentrum München, Klinische Kooperationsgruppe Molekulare Onkologie, München, Germany

Patients with hematologic malignancies relapsed after allogeneic stem cell transplantation may be treated with donor lymphocyte infusions resulting potentially in a beneficial graft versus leukemia effect. Selecting lymphocytes directed specifically towards defined tumor associated antigens (TAA) may improve this approach. We therefore previously isolated and characterized allorestricted CD8+ T cells and T cell receptors (TCR) with specificity for the TAA formin-related protein in leukocytes 1 (FMNL1) which is restrictedly expressed in hematopoietic cells and over expressed in different leukemias. However, CD4+ T cells with specificity for TAA have been rarely described and experiences with MHC class II-restricted TCR for transfer are highly limited. As it has been repeatedly shown that CD4+ T cells play a critical role in tumor eradication and maintenance of immune memory, the aim of this project was to isolate CD4+ T cells specifically recognizing FMNL1.

We used dendritic cells pulsed with recombinant protein or one out of nine peptide pools from an FMNL1 peptide-library for stimulation to isolate FMNL1-specific MHC class II-restricted T cells. After several rounds of restimulation, we were able to detect IFN-g responses of some T cell lines in response to targets either expressing FMNL1 or pulsed with the respective FMNL1 peptide pool. These T cell lines were then cloned by limiting dilution and further investigated by flow cytometry, IFN-g ELISA and Bioplex analysis.

Within the T cell clones, which have been stimulated with the peptide pool, we obtained several $\mathrm{T}$ cell clones highly specific for the same FMNL1 peptide. However, these clones did not recognize targets naturally presenting FMNL1. In contrast, using protein-pulsed dendritic cells as stimulator cells, we were able to generate CD4+ T cell clones reactive against cells naturally expressing FMNL1. Recognition of target cells could be improved by genetic transfer of FMNL1 and blocked by an HLA-DR specific antibody. Moreover preliminary data show, that one clone recognizes HLA-DR-matched chronic lymphocytic leukemia cells.

We were able to isolate HLA-DR-restricted CD4+ T cells with specificity for FMNL1 recognizing leukemic cells. Specific TCR have been isolated and are currently characterized after genetic transfer in indicator cell lines and PBMC

Disclosure: No conflict of interest disclosed.

P900

\section{FMR1NB represents a highly specific surface antigen in patients with acute myeloid leukemia}

Luetkens, T. ${ }^{1}$; Cao, Y. ${ }^{1}$; Bartels, K. ${ }^{1}$; Meyer, S. ${ }^{1}$; Bokemeyer, C. ${ }^{1}$; Atanackovic, $D{ }^{1}$

${ }^{1}$ University Medical Center Hamburg-Eppendorf, Hubertus-Wald

Tumorzentrum, Department of Oncology and Hematology, Hamburg,

Germany

Introduction: Their extraordinary tissue restriction renders Cancer-testis (CT) antigens ideal candidates for tumor-specific therapy. However, a pre dominantly intracellular localization of most CT antigens hampers the development of immediate targeted approaches. CT antigen FMR1NB might represent a promising exception as prediction algorithms indicate a putative transmembrane topology.

Methods: We screened acute (AML) and chronic (CML) myeloid leukemia samples as well as healthy tissues for the expression of FMR1NB. Mammalian cell lines were transfected with FLAG- or GFP-tagged FMR1NB expression constructs. We evaluated the subcellular localization of FMR1NB by fractionation and confocal microscopy. Epitopes of commercial anti-FMR1NB antibodies were characterized by ELISA using overlapping peptides, and localization and expression of the protein in AML cells was evaluated by immunofluorescence stainings and Western Blot.

Results: FMR1NB mRNA was expressed in AML cell lines and patient samples and confirmed by western blot on the protein level. Specificity was confirmed by the lack of FMR1NB expression in healthy tissues. Confocal fluorescence microscopy of GFP chimeras in mammalian cell lines, immunofluorescence staining of AML cell lines, and subcellular fractionation strongly support a membraneous localization of FMR1NB including the plasmamembrane. In contrast to results of structural prediction software our characterization of epitopes recognized by polyclonal anti-FMR1NB antibodies and the subsequent immunostaining of intact transfected and native AML cells suggest an extracellular amino-terminus of FMR1NB. Flow cytometric analysis of AML and CML cell lines as well as AML patient samples and healthy donors further confirmed a strong and specific expression of FMR1NB on leukemia samples.

Conclusions: FMR1NB mRNA is specifically expressed in AML cells and the resulting protein localizes to the membrane compartment including the plasma membrane. A putative extracellular domain of over 100aa length of the protein can be detected using FMR1NB-specific antibodies. The membrane localization of FMR1NB is a rare exception within the CT antigen family and our data suggest that it represents an ideal target for the diagnosis and therapy of myeloid leukemias.

Disclosure: No conflict of interest disclosed.

\section{P901}

\section{Relevance of receptor affinity for the adoptive $C D 8+T$ cell} therapy of HLA-A2/NY-ESO-1 positive tumors

\section{Jakka, G. ${ }^{1}$; Wadle, A. ${ }^{1}$; Kleber, S. ${ }^{1}$; Schuberth, P. ${ }^{1}$; Petrausch,} U. ${ }^{1}$; Hombach, A. ${ }^{2}$; Knuth, A. ${ }^{1}$; Renner, C. ${ }^{1}$; Mischo, A. ${ }^{1}$

${ }^{1}$ Universitätsspital Zürich, Klinik für Onkologie, Zürich, Switzerland, ${ }^{2}$ Klinik I für Innere Medizin und Zentrum für Molekulare Medizin der Universität zu Köln (ZMMK), Köln, Germany

The Cancer-testis (CT) antigen NY-ESO-1 is expressed in a variety of cancers but not in normal adult tissue except for the testis. The clinical goal of immunotherapy has traditionally been to provide either active or passive immunity against the malignant process. As NY-ESO-1 is a good tumorspecific antigen, it has been targeted by various immunotherapeutical strategies. It seems to be immunogenic as spontaneous humoral and CD8+ T-cell responses can be detected in some patients with advanced NY-ESO-1 expressing tumors. Effective cancer immunotherapy depends on the presence of sufficiently large number of CD8+ lymphocytes with anti tumor specificity and appropriate homing and effector functions, enabling recognition and destruction of cancer cells. Transfusion of ex vivo generated CD8+ T-cells with chimeric receptors (T-bodies) specific for the NY-ESO-1antigen is an alternative T-cell based tumor therapy.

In our study we expressed chimeric immune receptors on human CD8+ T-cells via retroviral mediated gene transfer. These receptors are composed of single chain variable fragments of various affinities binding to the NY-ESO-1 157165 peptide presented in the HLA-A2 complex fused to an intracellular signalling domain of CD28 and CD3 zeta.

Our results show differences in cytotoxicity and production of IFN-gamma in response to NY-ESO-1 expressing tumor cells with respect to their affinity.

Disclosure: No conflict of interest disclosed. 
P902

\section{T cell receptor gene transfer for the treatment of HER2 overexpressing breast cancer via adoptive $\mathrm{T}$ cell transfer}

Conrad, H. ${ }^{1}$; Brackertz, B. ${ }^{1}$; Meyerhuber, P. ${ }^{2}$; Kast, B. ${ }^{1}$;

Peschel, C. ${ }^{1}$; Uckert, $W^{2}$; Bernhard, $H^{1,3}$

${ }^{1}$ Klinikum rechts der Isar, III. Medizinische Klinik, München, Germany,

${ }^{2}$ Max-Delbrück-Center for Molecular Medicine, Berlin, Germany, ${ }^{3}$ Klinikum

Darmstadt, Medizinische Klinik V, Darmstadt, Germany

The clinical goal of our studies is the adoptive transfer of primary $\mathrm{T}$ cells transduced with HER2-specific T cell receptors (TCR) for patients with HER2-overexpressing breast cancer. Firstly, we established a panel of HER2reactive $\mathrm{T}$ cell clones as a source for the isolation of HER2-reactive TCR genes in order to redirect primary $\mathrm{T}$ cells toward HER2. Therefore, we generated tumor-reactive HER $2_{369-377}$ specific $\mathrm{T}$ cell clones either by stimulating HLA-A $2^{+} \mathrm{CD}^{+} \mathrm{T}$ cells with autologous HLA-A2 ${ }^{+}$dendritic cells (DC) transfected with HER2-mRNA, or by stimulating HLA-A2 CD8 $^{+} \mathrm{T}$ cells with allogeneic HLA-A2 ${ }^{+}$DC loaded with the peptide HER2 $2_{369-377}$. HER2 reactive $\mathrm{T}$ cells were screened via ELISpot and FACS sorting, respectively, cloned and further tested in ${ }^{51}$ Chromium release assays, ELISA or proliferation assays using HER2 peptides pulsed on as well as HER2-overexpressing cell lines.

Retroviral constructs containing a HER2-specific TCR either from the autologous or the allogeneic approach were transduced into $\mathrm{CD}^{+} \mathrm{T}$ cells and compared with each other and their respective parental clones in different functional tests. To enhance the TCR expression its TCRalpha and beta chain were linked via P2A, its constant regions were murinized and it was codon optimized. As a control for cardiotoxicity TCR-transduced T cells were tested against $\mathrm{A} 2^{+} \mathrm{HER} 2^{+}$cardiomyocytes.

The $\mathrm{CD}^{+} \mathrm{T}$ cells transduced with the optimized TCR showed a better recognition than the T cells with the non-optimized TCR consistent with the parental $\mathrm{T}$ cell clone. The allogeneic $\mathrm{T}$ cell clone as well as the respective TCRtransduced $\mathrm{T}$ cells recognized not only

HER $2_{369-377}$ but also the corresponding peptides derived from HER3 and HER4. The autologous CTL clone and the respective TCR-transduced T cells recognized only HER $2_{369-377 .}$ The cardiomyocytes were not recognized. Our results are very promising for the HER2-directed design of immunotherapies based on TCR-transduced T cells. Depending on whether HER2 or HER2 and HER3/HER4 are overexpressed different TCR are at disposal. First tests show no cardiotoxicity in vitro. Additionally, one of the TCR is currently tested in a preclinical in vivo model.

Disclosure: No conflict of interest disclosed.

\section{P903}

\section{Generation of CAR-transgenic T and NK cells to enhance cell-mediated cytotoxic activity against erbB2/HER2neu- expressing tumors}

\section{Rudzki, J.D. ${ }^{1}$; Rumpold, H. ${ }^{2}$; Gastl, G. ${ }^{1}$; Wolf, D. ${ }^{1}$}

${ }^{1}$ Medizinische Universität Innsbruck, Innere Medizin V, Hämatologie und Onkologie, Innsbruck, Austria, ${ }^{2}$ Krankenhaus der Barmherzigen Schwestern Linz, Innere Medizin I, Hämatologie und Onkologie, Linz, Austria

Introduction: Cellular-based therapies have become an interesting alternative to target malignant diseases. Infusion of genetically engineered immune cells is a highly innovative approach to target tumor cells. Chimeric antigen receptor (CAR) expressing cells enable tumor-cell killing in a MHC-I independent manner contrary to $\mathrm{T}$ cell receptor overexpressing cytotoxic T cells (CTL). Apparently, the fact that CAR transgenic (CARtg) CTL are clinically not as potent as expected emphasizes the need for the development of novel concepts to improve adoptive $\mathrm{T}$ cell transfers.

Methods: In order to obtain high viral titers 293FT cells were transfected with a gateway-compatible $3^{\text {rd }}$ generation lentiviral vector system to overexpress a CAR which comprises a scFV-domain recognizing erbB2/HER2neu linked to costimulatory elements (mu-/hu-CD28, huCD3zeta). CRAs (chromium release assays) were carried out to determine the efficacy of CARtg cells in mediating tumor cell lysis of a HER2-positive as well as HER2-negative MCF7 cell line. Moreover, novel NK-CAR for specific enhancement of CARtg human NK cell-induced cytotoxicity are established with the ultimate goal to increase anti-tumor efficacy by combining CARtg T cell subpopulations together with NK cells.

Results: Viral titers as calculated by real time PCR reached up to $3.8 \times 10^{+10}$ viral particles. High efficient transduction of human cell lines (Jurkat, K562) were obtained by using the control vector (pCCL_g_EGFP) and the major target vector (pCCL_g_CAR)(i.e. EGFP: $87 \%$ of Jurkat/96\% of K562; CAR: $84 \%$ of Jurkat/97\% of K562). Subsequently selected primary human T cells $\left(\mathrm{CD}^{+}: 74.7 \%\right.$; $\left.\mathrm{CD}^{+}: 80.4 \%\right)$ were lentivirally tranduced and further enriched by MACS selection $\left(\operatorname{tgCD} 4^{+}: 95.8 \% ; \operatorname{tgCD} 8^{+}: 93.1 \%\right)$, thus enabling an effective target lysis of $37 \%-25 \%(\mathrm{E}: \mathrm{T}=100: 1-10: 1)$ after short-term in vitro expansion ( $\operatorname{tgCD} 8^{+} \mathrm{T}$ cells alone).

Conclusions: Ongoing experiments are performed to test out the helper function of CARtg CD4 $4^{+} \mathrm{T}$ cells in augmenting antitumor response together with CARtg effectors like $\mathrm{CD}^{+} \mathrm{T}$ or NK-Cells. A novel set of NK-CARs containing HER2/neu-reacting single chain domains linked to NK cell specific stimulatory elements (NKG2D with DAP10 or h2B4) will amend the existing CAR. It is the final goal of this project to generate CARtg lymphocyte subpopulations to target HER2/neu expressing tumors using a combination of these cell fractions, as each population has been shown to have limited anti-tumor potential.

Disclosure: No conflict of interest disclosed.

\section{P904}

\section{Radiotherapy may potentiate immunotherapy through presentation of novel tumor antigens}

\section{Sharma, A. ${ }^{1}$; Bode, B. ${ }^{2}$; Knuth, A. ${ }^{1}$; van den Broek, M. ${ }^{1}$; von}

\section{Boehmer, $L^{1}{ }^{1}$}

${ }^{1}$ Universitätsspital Zürich, Onkologie, Zürich, Switzerland,

${ }^{2}$ Universitätsspital Zürich, Pathologie, Zürich, Switzerland

Introduction: Radiotherapy is a standard modality in cancer treatment. Recent studies have shown that therapeutic irradiation not only enhances antigen presentation by MHC class I molecule upregulation, but also results in the presentation of novel epitopes derived from radiation induced proteins. Cancer testis antigens (CTAs) are promising targets for immunotherapy, due to their restricted expression to germ cells and many cancer types. Spontaneous humoral and cell-mediated immune responses have been demonstrated against several CTAs, which illustrates their high immunogenicity. We propose that radiation induces de novo expression and upregulation CTAs in cancer tissues.

Methods: To test the hypothesis, we irradiated multiple cancer cell lines with gamma-radiation from a ${ }^{60} \mathrm{Co}$ source. The cells were harvested at different time points after different doses of radiation and the induction of CTAs and MHC class I expression was analyzed by real-time PCR and immunofluorescence. To further verify our findings, we irradiated human tumor biopsies of different cancer types in vitro and also obtained tissue samples for immunohistochemistry from sarcoma patients before and after neoadjuvant radiotherapy (in vivo)

Results: We found that irradiation induced the expression of CTAs in a dose and time dependent manner in multiple CTA negative cancer cell lines. In CTA positive cell lines an upregulation of the antigens was observed. MHC class I molecules were upregulated upon irradiation. In the tumor biopsies irradiated ex vivo the expression of CTAs and MHC class I molecules was induced and upregulated. These results were confirmed in the immunohistochemical stainings from sarcoma patients treated with neoadjuvant radiotherapy. Interestingly, in these patients a dense immune infiltrate in the tumor tissue was observed after radiotherapy.

Conlusion: Radiotherapy may enhance immune recognition of cancer cells. A combination of radio- and immunotherapy may therefore become a promising new approach to cancer treatment.

Disclosure: No conflict of interest disclosed 
P905

\section{Advanced stage of malignant melanoma corresponds significantly to elevated MelanA-specific and PD-1+ ${ }^{+}{ }^{+} 8^{+}$ T cells}

Krönig, H. ${ }^{1}$; Falchner, K.J. ${ }^{1}$; Odendahl, M.2; Muck, D. ${ }^{3} ;$ Blank, C.4; Peschel, C. ${ }^{1}$; Haller, B. ${ }^{5}$; Schulz, S. ${ }^{6}$; Bernhard, H. ${ }^{1,7}$

${ }^{1}$ Technische Universität München/ Klinikum Rechts der Isar, Department of Hematology/Oncology, München, Germany, ${ }^{2}$ Technische Universität München/ Klinikum Rechts der Isar, Institute of Microbiology, Immunology and Hygiene, München, Germany, ${ }^{3}$ Technische Universität München/ Klinikum Rechts der Isar, Department of Dermatology, München, Germany, ${ }^{4}$ The Netherlands Cancer Institute - Antoni van Leeuwenhoek Hospital (NKI-AVL), Division of Immunology and Department of Medical Oncology, Amsterdam, Netherlands, ${ }^{5}$ Technische Universität München/ Klinikum Rechts der Isar, Institute of Medical Statistics and Epidemiology, München, Germany, ${ }^{6}$ Technische Universität München/ Klinikum Rechts der Isar, Department of Pathology, München, Germany, ${ }^{7}$ Klinikum Darmstadt, Department of Hematology/Oncology, Darmstadt, Germany

The PD-1 pathway is emerging as an important tumor-evasion mechanism. It has been postulated that tumor cells may upregulate PD-L1 in order to inhibit cytotoxic T cells expressing the corresponding receptor PD-1. We investigated the frequency of PD-1 expressing peripheral MelanA-specific T cells subject to the tumor stage of melanoma patients and their possible prognostic relevance for overall survival.

100 samples of PBMCs from HLA- $2^{+}$patients with malignant melanoma were analyzed in 5 colour FACS. This was combined with tetramer analyses for accurate phenotyping. After gating on $\mathrm{CD}^{+} / \mathrm{CD}^{+}$lymphocytes, staining with an HLA-A2/MelanA-tetramer was performed. Subsequently we analyzed PD-1 expression on MelanA-specific $\mathrm{CD}^{+} \mathrm{T}$ cells depending on the tumor stage. Corresponding formalin-fixed paraffin-embedded tissues of primary tumor and distant organ metastases from 37 cases was analyzed by immunohistochemistry for MelanA, PD-L1 and PD-1 expression.

The frequency of MelanA-specific $\mathrm{CD}^{+} \mathrm{T}$ cells increased significantly from stage I to IV $(\mathrm{p}<.001)$. Moreover the PD-1 expression on MelanA-specific $\mathrm{CD}^{+} \mathrm{T}$ cells was overrepresented in stages III and IV compared to all CD8 $\mathrm{T}$ cells $(\mathrm{p}<.001)$ and compared to the local stages I and II $(\mathrm{p}=0.035)$ Consistent with these data we found a tendency to higher PDL-L1 expression in the advanced tumor stages III and IV. However, the increased PD-1 and PD-L1 expression was not associated with overall survival.

Our results suggest that the PD-1 expression occurred concerted on the tumor antigen-specific $\mathrm{T}$ cells in the context of the disease, which increased with the advanced tumor stage. The increased expression of PD-1 on MelanA-tetramerpositive $\mathrm{CD}^{+} \mathrm{T}$ cells had no effect on overall survival which could be due to other immune escape mechanisms such as the induction of $\mathrm{T}$ cell anergy, regulatory $\mathrm{T}$ cell mediated $\mathrm{T}$ effector inhibition, myeloid derived suppresso cells (MDSC) and stromal protection. Furthermore there was a tendency to higher PD-L1 expression in the tumor stages III and IV consistent with the significant higher PD-1 levels on $\mathrm{CD}^{+} \mathrm{T}$ cells in the peripheral blood Interestingly the Kaplan Meyer curves yield even an insignificant tendency of higher overall survival in patients who expressed higher PD-L1 on thei tumors. This indicates that PD-L1 mediated inhibition may also occur between cell partners other than effector T cells and tumor cells, such as during the interaction between APC and regulatory T cells.

Disclosure: No conflict of interest disclosed.

\section{P906}

\section{The immune inhibitory receptor osteoactivin is up-regulated in monocyte-derived dendritic cells by tyrosine kinase inhibitors}

Schwarzbich, M.-A. ${ }^{1}$; Schmidt, S.M. ${ }^{1}$; Gutknecht, M. ${ }^{1}$; Salih, J. ${ }^{1}$; Kanz, L. ${ }^{1}$; Salih, H.R. ${ }^{1}$; Grünebach, F.

${ }^{1}$ University of Tübingen, Internal Medicine II, Division of Oncology, Hematology, Immunology, Rheumatology and Pneumology, Tübingen, Germany

Introduction: Osteoactivin (glycoprotein nonmetastatic B (GPNMB) DC-HIL) is differentially expressed in human dendritic cells (DC), and expression on antigen presenting cells can inhibit $\mathrm{T}$ cell activation by binding syndecan-4 (SD-4). Tyrosine kinase inhibitors (TKI) display impressive anticancer effects. The TKI imatinib is successfully used in the treatment of chronic myeloid leukemia (CML) and gastrointestinal stroma tumors. Dasatinib and nilotinib represent second generation TKI for the treatment of CML. However, it has been shown that these agents also affect the differentiation and function of DC. Here we investigated the effect of TKI on the expression of osteoactivin in human in vitro generated DC.

Methods: DC were generated from blood monocytes by plastic adherence and exposure to GM-CSF and IL-4. LPS was added on day 6 of culture for maturation of DC. Imatinib, nilotinib or dasatinib were added to the culture medium every second day starting from the first day of culture. Cells were harvested for immunophenotyping and osteoactivin expression analysis by immunostaining, western-blotting and real-time RT-PCR or for mixed lymphocyte reactions (MLR) on day 7 of culture.

Results: DC generated in the presence of therapeutic concentrations of the TKI showed a significantly reduced expression of CD1a, CD83, the co-stimulatory molecule CD86 and DC-SIGN. Furthermore, we found excessive upregulation of osteoactivin on TKI-treated DC. Interestingly, incubation with the immunosuppressive and anti-inflammatory cytokine IL-10 also resulted in osteoactivin over-expression. In line with osteoactivin up-regulation, exposure to TKI resulted in reduced stimulatory capacity of DC in MLR with allogenic T cells

Conclusions: Osteoactivin is up-regulated in human DC by TKI or immunosuppressive cytokines indicating that up-regulation of osteoactivin may be involved in the inhibition of DC function upon TKI exposure. This is supported by the fact that the immunosuppressive cytokine IL-10 up-regulated osteoactivin expression. Our findings are of importance for future combinatory approaches using TKI and DC-based immunotherapy and indicate that inhibition of osteoactivin expression or function may serve as a novel strategy for immunotherapeutic interventions in cancer patients.

Disclosure: No conflict of interest disclosed.

\section{P907}

\section{Lenalidomide combined with dasatinib shows long-term but no synergistic short-term effects on direct NK-cell activity against MM cells in vitro}

\section{Zekl, M. ${ }^{1}$; Stühmer, . $^{1}$; Einsele, H. ${ }^{1}$; Seggewiß, R.}

${ }^{1}$ Department of Internal Medicine II, University of Würzburg, Hematology/ Oncology, Würzburg, Germany

Introduction: Lenalidomide, an IMiD $®$ (immunomodulatory drug), is established for the treatment of relapsed/refractory multiple myeloma (MM) in combination with dexamethasone. It modulates long-term natural killer (NK) cell effector functions by induction of IL-2 production. The BCR-ABL/SRC inhibitor dasatinib blocks SRC kinases relevant for signal transduction in MM and NK cells. A clinical trial evaluates the combination of lenalidomide/dasatinib/dexamethasone for the treatment of MM (NCT00560391). We investigated in vitro effects of lenalidomide \pm dasatinib against MM cells and on NK cell activity targeting MM.

Methods: Analysis of drug effects on human PBMCs or expanded NK cells included apoptosis/necrosis induction, cytotoxicity, degranulation marker $\mathrm{CD} 107 \mathrm{a} / \mathrm{b}$ expression and cytokine production. Evaluation of direct drug effects on primary MM cells and cell lines U266 and OPM-2 included proliferation and apoptosis measurements.

Results: Dasatinib \pm lenalidomide increased the apoptosis/necrosis rate in PBMCs $(n=4, p<0.05)$, but decreased it in expanded NK cells $(n=5, p<0.05)$ 5 days lenalidomide $+24 \mathrm{~h}$ dasatinib pretreatment of PBMCs and then washout of the drugs led to enhancement of IL-2 production and NK cells CD107a/b expression, when stimulated with MM cell lines. In contrast, $24 \mathrm{~h}$ pretreatment of NK cells with lenalidomide followed by wash-out of the drug had no effect on cytotoxicity, CD107a/b expression and cytokine production. However, 24h pretreatment of expanded NK cells with dasatinib \pm lenalidomide reduced the cytotoxicity against MM cell lines $(n=3, p<0.05)$. When the drugs were present in the assay, but NK cells not pre-treated, cytotoxicity was also inhibited $(n=3, p<0.05)$. Apoptosis induction in primary MM cells in co-culture with stromal cells was heterogeneous with 4/12 samples showing an enhancement by dasatinib + lenalidomide, 4/12 samples no improvement over the dasatinib activity alone, and the remaining samples unaffected by 
either the single drugs or their combination. Lenalidomide + dasatinib was more effective than the single drugs alone in OPM-2 cells \pm coculture with stromal cells, but had little effect on U266 cells.

Conclusions: Lenalidomide combined with dasatinib does not increase shortterm NK cell activity against MM cells, although some activity was observed long-term. The anti-proliferative dasatinib effect against MM cells is not in all cases enhanced by lenalidomide in vitro. Further evaluations are warranted.

Disclosure: Michael Zekl: No conflict of interest disclosed.

Ruth Seggewiß: Honoraria: Vortragstätigkeit für Bristol-Myers-Squibb

\section{P908}

\section{Spontaneous WT1 specific T cell responses in patients with HCC and chronic liver diseases}

Döring, S. ${ }^{1}$; Scheibenbogen, C. ${ }^{2}$; Somasundaram, R. ${ }^{3}$; Klugewitz, K. ${ }^{3}$; Stather, D. ${ }^{1}$; Seehofer, D. ${ }^{4}$; Pascher, A. ${ }^{4}$; Daum, S. ${ }^{3}$; Neuhaus, P. ${ }^{4}$; Zeitz, M. ${ }^{3}$; Thiel, E. ${ }^{1}$; Keilholz, U. ${ }^{1}$; Letsch, $A{ }^{1}$

${ }^{1}$ Hämatologie und Onkologie, Charité CBF, Berlin, Germany, ${ }^{2}$ Institut für Medizinische Immunologie, Charité Campus Mitte, Berlin, Germany,

${ }^{3}$ Gastroenterologie, Infektiologie und Rheumatologie, Charité CBF, Berlin, Germany, ${ }^{4}$ Klinik für Allgemein-, Visceral- und Transplantationschirurgie, Charité Campus Virchow Klinikum, Berlin, Germany

Introduction: Immunotherapy approaches are currently proposed in hepatocellular carcinoma (HCC) and appear promising in treatment and prevention of HCC. Tumor-specific antigens identified in HCC demonstrated immunogenicity both in preclinical and clinical trials. In addition to the antigens analysed so far, one promising target antigen for immunotherapy in HCC is the transcription factor Wilms tumor gene product 1 (WT1), which is overexpressed in HCC and may play an important role in carcinogenesis and prognosis of HCC. We analysed WT1 specific T cells spontaneously occurring in patiens with HCC and chronic liver disease.

Methods: PBMC from healthy controls $(n=6)$, patients with chronic hepatitis $(n=20)$, patients with chronic liver cirrhosis $(n=20)$ and patients with HCC $(n=20)$ were analysed direct ex vivo for spontaneous CD4 and CD8 T-cell responses after stimulation with a WT1-peptide-mix by multicolor flow cytometry.

Results: After stimulation with WT1 peptide-mix IFNg and/or TNFa -producing CD8+ WT1-specific T cells could be detected in 8 of 19 HCC patients $(42 \%)$, in 3 of 21 patients with liver cirrhosis (14\%) and in 2 of 21 patients with chronic hepatitis (10\%). Cytokine secreting CD4+ WT1 specific T cells were found in 5 of $19 \mathrm{HCC}$ patients $(26 \%)$, in 4 of 21 patients with liver cirrhosis (19\%) and in 3 of 21 patients with chronic hepatitis (14\%). Frequencies of CD8+ and CD4+ WT1 specific T cells ranged between $0.24 \%$ and $5.5 \%$ of cytokine producing $\mathrm{T}$ cells and were similar for both cytokines, $\mathrm{T}$ cell subtypes and disease subgroups. In none of the healthy controls WT1 specific CD8+ or CD4+ cytokine+ $\mathrm{T}$ cells could be detected after stimulation with the WT1 peptide-mix.

Conclusion: These results provide for the first time evidence for spontaneous T-cell reactivity against WT1 in patients with HCC and to a lower extend also in patients with chronic liver disease. These data support the immunogenicity of WT1 in HCC and the potential usefulness of this antigen for HCC immunotherapy.

Disclosure: No conflict of interest disclosed.
P909

Optimized activation of T-cells with specificity for the human telomerase reverse transcriptase (hTERT) in patients with advanced non-small cell lung cancer (NSCLC)

Gahn, B. ${ }^{1}$; Wellnitz, D. ${ }^{1}$; Wöster, K. ${ }^{1}$; Staudinger, M. ${ }^{2}$; Böttcher, S. ${ }^{1}$; Gramatzki, M. ${ }^{2}$; Kneba, M. ${ }^{1}$

${ }^{1}$ Universitätsklinikum Schleswig Holstein Campus Kiel, II.Medizinische Klinik, Kiel, Germany, ${ }^{2}$ Universitätsklinikum Schleswig Holstein Campus Kiel, II.Medizinische Klinik, Sektion für Stammzelltransplantation und Immuntherapie, Kiel, Germany

Introduction: The human telomerase reverse transcriptase (hTERT) is expressed in $93 \%$ to $100 \%$ of non small cell lung cancer (NSCLC), making the enzyme a potential target for specific immunotherapy. Present vaccination studies show a survival advantage in patients with a hTERT-specific immune response (Kosmatopoulos et al., 2007), but the number of immune responders is still limited. In the current study we tried to improve the activation of hTERT-specific T-cell responses in NSCLC-patients of different HLA-types who simultaneously received platinum based standard $1^{\text {st }}$ line chemotherapy protocols.

Methods: After depletion of regulatory T-cells the remaining lymphocytes were stimulated in vitro with mature peptide loaded dendritic cells (DC). The DC were either matured with the standard cytokine cocktail consisting of GM-CSF, IL-4, IL-6, IL- $1 \beta$, TNF- $\alpha$ and PGE $_{2}$ or with a TLR7/8- in combination with a CD40-ligand. The activation of hTERT-specific T-cells was measured by flow cytometric analysis of intracellular IFN- $\gamma$.

Results: In 2 patients the hTERT-specific T-cell response was more intensively activated by cytokine matured DC than by TLR7/8- and CD40-L matured DC. Using this standard approach $0,1-0.6 \% \mathrm{IFNg}^{+} \mathrm{CD} 8^{+} \mathrm{T}$-cells cells were activated.

In contrast in 3 other patients hTERT-specific T cells could be more effectively activated by TLR7/8- and CD40-L matured DC. We could activate between $0,1 \%$ and $2,1 \% \mathrm{IFN}^{+} \mathrm{CD} 8^{+}$T-cells.

Similarly in one healthy donor the hTERT-specific T-cell response was more intensively activated by cytokine matured DC showing up to $0,5 \% \mathrm{IFNg}^{+}$ $\mathrm{CD}^{+} \mathrm{T}-$ cells. However in another healthy donor hTERT-specific T-cell responses could be more effectively activated by TLR7/8- and CD40-L with up to $0.7 \% \mathrm{IFNg}^{+} \mathrm{CD}^{+}$cells.

In 2 other patients we could not activate any hTERT-specific IFNg $^{+}$T-cell response.

Conclusion: These data indicate that by using different DC maturation protocols hTERT-specific T-cells can be activated in the majority of patients. Even concomitant to standard chemotherapy clinical vaccination protocols may be feasible. However DC maturation protocols may need to be individualized. The very low percentage of hTERT-specific T-cells in some patients implicate the need for even more potent immune adjuvants.

Disclosure: No conflict of interest disclosed.

P910

\section{Lymphoblastoid cell lines as potential vaccines for the immunotherapy of cancer testis antigen-expressing tumors}

Kaddu-Mulindwa, D. ${ }^{1}$; Neumann, F. ${ }^{1}$; Kubuschok, B. ${ }^{1}$;

Pfreundschuh, M. ${ }^{2}$

${ }^{1}$ Universität des Saarlandes, José Carreras Zentrum für Immuntherapie und Gentherapie, Homburg, Germany, ${ }^{2}$ Universitätsklinikum des Saarlandes, Innere Medizin I, Homburg, Germany

Introduction: Cancer germ line or cancer testis antigens (CTA) are expressed in a broad spectrum of human tumors, but not in normal tissues, except for germinal cells in the testis and ovary. Due to this expression pattern CTAs are promising candidates for immunotherapeutic approaches in haematological malignancies and solid tumors. EBV-transformed lymphoblastoid cell lines (LCL) have been shown to be potent antigen-presenting cells. To investigate whether LCLs can be used as anti-cancer testis antigen vaccines, we studied the composite expression of 12 CTAs in 20 LCLs. 
Methods: The existence of mRNA of these $12 \mathrm{CT}$ genes was assessed by RT-PCR. Moreover, if the corresponding antibodies were available, CTA expression also was analyzed by Western Blot and immunostaining.

Results: The most frequently expressed CT genes were SSX4 (10/20 or 50 $\%)$, followed by GAGE (9/20 or $45 \%)$. SSX1 was expressed in $7 / 20(35 \%)$, MAGE-A3 in 5/20 (25\%) and SSX2 in 4/20 LCL (20\%). mRNA encoding the CT antigens MAGE-C1, SCP1, MAGE-C2, and HOM-TES-85 was expressed in 3/20 LCL (15\%). MAGE-A4 and NY-ESO-1 could be found in one LCL in each case (5\%). BORIS (CT 27) was not detected in any of the 20 LCL tested. Fourteen of 20 LCL (70 \%) expressed at least one antigen; 9 (45\%) LCL expressed $\geq 2$ CT genes, and 4/20 LCL (20\%) expressed at leas $7 / 10$ of the CT genes analyzed in this study. The expression of CT genes did not correlate with the length of in vitro culture, telomerase activity, aneuploidy, proliferation or copy numbers of the Latent Membrane Protein 1 (LMP1) or DNA methyltransferase 1 (DNMT1) per cell.

Conclusion: The stability of multiple CT gene expression over prolonged culture periods makes LCL attractive candidates for CT vaccines. In addition, EBV-transformed LCL represent an ideal model for studying in more details the mechanisms underlying the expression of $\mathrm{CT}$ genes during malignant transformation.

Disclosure: No conflict of interest disclosed.

\section{P911}

\section{Influence of Lenalidomide on NK-cell mediated antibody- dependent cellular cytotoxicity}

\author{
Friedrichs, B. ${ }^{1}$; Bruns, C. ${ }^{1}$; Blau, W. ${ }^{1}$; Thiel, E. ; Uharek, L. \\ ${ }^{1}$ Charite Universitätsmedizin Berlin - Campus Benjamin Franklin, \\ Hämatologie/Onkologie/Transfusionsmedizin, Berlin, Germany
}

Introduction: Treatment options of hematological malignancies have been expanded by the introduction of immunomodulatory drugs (IMiDs), i.e. Lenalidomide, and the increasing use of monoclonal antibodies (mABs). NK cells are the major effector cell population for antibody-dependent cellular cytotoxicity (ADCC). Although the exact mechanism of action of IMiDs is not known, it is postulated that modulation of NK cell function is one of their properties. In order to characterize the effect of IMiDs on NK cells, a series of stimulation assays was performed in vitro. Patients undergoing Lenalidomide treatment for multiple myeloma (MM) and chronic lymphocytic leukemia (CLL) were monitored for ADCC and NK cell activation markers.

Methods: CD56+-selected NK cells of healthy donors (HD) and patients with MM ( $n=3)$ and CLL $(n=1)$ were obtained. HD NK cells were incubated for $48 \mathrm{~h}$ with Lenalidomide in varying concentrations $(0,5 \mu \mathrm{mol} / \mathrm{ml} ; 1 \mu \mathrm{mol} / \mathrm{ml}$ $10 \mu \mathrm{mol} / \mathrm{ml}$ ). Lenalidomide-stimulated NK cells were co-incubated with K562 cells for analysis of natural cytoxicity. For assessment of ADCC NK cells were co-incubated with Raji cells (CD20+/CD40+ lymphoma cell line) and CD20antibody Rituximab and CD40-antibody HCD122 in a 4h CD107a assay FACS analysis included IFNg- and TNFa-production, NK cell markers NKG2D, NKp30, NKp44, and NKp46 and CD107a as a marker for NK cell degranulation. Lysis of target cells was analyzed in a standard calcein labeling assay.

Results: For ADCC, the percentage of degranulating NK in cells was significantly increased when the effector NK cells were preincubated with Lenalidomide $((20 \% \pm 3 \%$ without Lenalidomide stimulation vs. $41 \% \pm 4 \%$ after Lenalidomide preincubation). Incubation of the target cell line Raji did not lead to an additional lysis. Intracellular production of IFNg and TNFa was increased in Lenalidomide-pretreated NK cells after CD16 stimulation, but no increase of natural NK cell cytoxicity was observed. Three out of four patients undergoing Lenalidomide treatment showed an increase of $20 \% \pm 4 \%$ of NK-cell mediated ADCC on $\mathrm{d}+15$ while no changes in natural cytoxiticity were observed. We did not see an increase in expression of NK activation markers.

Conclusions: In vitro, Lenalidomide increases ADCC mediated by NK cells in response to two different antibodies. First results of patient monitoring during treatment support these findings and larger cohort will be examined. Use of IMiDs and mABs may be a synergistic treatment option.

Disclosure: No conflict of interest disclosed.

\section{P912}

\section{UV exposure boosts transcutaneous immunization and} improves tumor immunity: Cytotoxic T cell priming via the skin

Stein, P. ${ }^{1}$; Rechtsteiner, G. ${ }^{1,2}$; Warger, T. ${ }^{1,3}$; Fuhr, T. $^{1}$; Probst, H.-C. ${ }^{1}$; Stassen, M. ${ }^{1}$; Langguth, P. ${ }^{4}$; Schild, H. ${ }^{1}$; Radsak, M.P. ${ }^{1,5}$

${ }^{1}$ Institut für Immunologie, Universitätsmedizin, Mainz, Germany, ${ }^{2}$ Fresenius Medical Care, Bad Homburg, Germany, ${ }^{3}$ Schering-Plough, Dept. Immunology, Zürich, Switzerland, ${ }^{4}$ Institut für Pharmazie, Johannes Gutenberg-Universität, Mainz, Germany, ${ }^{5}$ III. Medizinische Klinik, Universitätsmedizin, Mainz, Germany

Background: Immunologic approaches to combat cancer aim at the induction of tumor reactive immune responses to achieve long-term protection. In this context, we recently developed a transcutaneous immunization (TCI) method using the toll-like receptor 7 agonist imiquimod and a peptide epitope. Application onto intact skin induces potent cytotoxic $\mathrm{T}$ lymphocyte (CTL) responses and protection against transplanted tumors in an experimental mouse model.

Purpose: The intention of this study was to explore the effects of UV irradiation on imiquimod based TCI.

Results: Here we show that skin exposure to low dose ultraviolet light before TCI with imiquimod strongly boosts specific CTL responses leading to memory formation and enhanced tumor protection. Towards the mechanisms, we demonstrate the activation of bone marrow derived dermal Dendritic cells (DC), but not Langerin expressing DCs are responsible for enhanced CTL activation.

Conclusions: We describe an optimized TCI method that mediates enhanced CTL and anti-tumor responses by a DC and TLR dependent mechanism. These data may provide the basis for the future development of advanced vaccination protocols against tumors and persistent virus infections.

Disclosure: No conflict of interest disclosed.

\section{P913}

\section{Functional dendritic cells can be generated from the peripheral blood of patients with glioblastoma even during treatment with temozolomide}

\section{Xun, X. ${ }^{1}$; Stockhammer, F.2 ; Mani, J. ${ }^{1}$; Schmitt, A. ${ }^{1}$;} Casalegno-Garduno, R. ${ }^{1}$; Freund, M. ${ }^{1}$; Schmitt, M. ${ }^{1}$

${ }^{1}$ Universität Rostock, Klinik für Innere Medizin III, Hämatologie und Onkologie, Rostock, Germany, ${ }^{2}$ Universität Rostock, Klinik für Neurochirurgie, Rostock, Germany

Introduction: The median survival of patients with glioblastoma remains poor despite the combination of surgery, radiotherapy and chemotherapy. There is a fervent need for innovative immunotherapies. such as dendritic cell (DC) vaccination to enhance T cell-mediated anti-tumor immunity. The basis of DC therapy is the generation of sufficient and functional autologous DCs. The methylating agent temozolomide (TMZ) is widely used for malignant glioma chemotherapy. Prior to applying DC therapy, the influence of TMZ on the phenotype and function of DCs needs to be defined.

Methods: An in vitro DC culture protocol with IL-4, GM-CSF and TNF alpha was followed to generate DCs from the peripheral blood of 20 healthy donors (HDs) and 20 patients (Pts) suffering from glioblastoma. DCs were phenotyped by flow cytometry and the morphology of the cells was analyzed by light microscopy. Both day +6 immature DCs and day +8 mature DCs were collected and subsequently exposed to FITC-dextran $(1 \mathrm{mg} / \mathrm{ml})$ and examined by flow cytometry. TMZ was added in DC culture medium on day 0 of DC generation at a concentration of $0,50,100,250$ or $500 \mu \mathrm{M}$

Results: On day +8 , cells from both groups in the monocyte displayed a typical DC morphology with cytoplasmic veils and a mature DC phenotype with coexpression of CD86 and CD83 in 45\% (Median) of the cells and high expression of HLA-DR in $99 \%$ of the cells. Expression of CD11c (90\% vs. $79 \%)$ and CD209 (72\% vs. 51\%) was even higher in DCs generated from Pts than from HDs. In contrast expression of CD40 (1.5\% vs. $12 \%)$ and CD80 (25\% vs. $42 \%$ ) was lower in Pts. DC precursors from monocytes of Pts and HDs also showed typical veils and colony formation in DC medium by phase contrast 
microscopy on day+6. Endocytic activity was shown in immature DCs generated from the peripheral blood of both HDs and Pts. Monocytes from HDs exposed with 250 or $500 \mu \mathrm{M}$ TMZ containing medium for 6 days showed a significant dose-dependent inhibition of the generation of CD83+ mature DCs (Median: $23.2 \%$ vs. $11.5 \%$ ) when compared with $0 \mu \mathrm{M} \mathrm{TMZ}$ group (49.3\%). Conclusion: In summary, mature DCs can be generated from glioblastoma Pts as well as from HDs. These DCs are characterized by up-take of FITC-dextran and by a high expression of HLA-DR, CD86, CD83 and CD209, even cocultured with 50-100 $\mu \mathrm{M}$ TMZ. Higher concentrations $(250$ or $500 \mu \mathrm{M})$ of TMZ hamper generation of autologous DCs. However, TMZ levels in the serum of patients under TMZ therapy do usually not exceed $50 \mu \mathrm{M}$.

Disclosure: No conflict of interest disclosed.

\section{P914}

Allogeneic gene-modified tumor cells (RCC-26/IL-7/CD80) as a vaccine in patients with metastatic renal cell cancer (RCC) - a clinical phase-I study

Flörcken, A. ${ }^{1}$; Willimsky, G. ${ }^{2,3}$; Kopp, J. ${ }^{1}$; van Lessen, A. ${ }^{1}$; Takvorian, A. '; Jöhrens, K. ${ }^{4}$ 'Lukowsky, A. ${ }^{5}$ 'Schönemann, C. ${ }^{6}$; Sawitzki, B. ${ }^{7}$; Pohla, H. ${ }^{8,9}$; Frank, R. ${ }^{10}$; Dörken, B. ${ }^{1,3}$; Schendel, D.J. ${ }^{9}$; Blankenstein, T. ${ }^{3}$; Westermann, J. ${ }^{1,3}$; Pezzutto, A. ${ }^{1,3}$

${ }^{1}$ Dept. of Hematology, Oncology and Tumor Immunology, Campus Virchow-Klinikum, Charité University Medicine, Berlin, Germany, ${ }^{2}$ Institute of Immunology, Campus Benjamin Franklin, Charité University Medicine, Berlin, Germany, ${ }^{3}$ Max Delbrück Center for Molecular Medicine, Berlin, Germany, ${ }^{4}$ Dept. of Pathology, Campus Mitte, Charité University Medicine Berlin, Germany, ${ }^{5}$ Dept. of Dermatology, Campus Mitte, Charité University Medicine, Berlin, Germany, ${ }^{6}$ Dept. of Transfusion Medicine, Campus Mitte Charité University Medicine, Berlin, Germany, ${ }^{7}$ Institute of Immunology, Campus Mitte, Charité University Medicine, Berlin, Germany, ${ }^{8}$ Laboratory of Tumor Immunology, LIFE Center, Ludwig-Maximilians-University, Munich, Germany, ${ }^{9}$ Institute of Molecular Immunology, Helmholtz Zentrum Munich, German Research Center for Environmental Health and Clinical Cooperation Group 'Immune Monitoring', Munich, Germany, ${ }^{10}$ Dept. of Chemical Biology, Helmholtz Center for Infection Research, Braunschweig, Germany

Introduction: Cellular immunotherapy aiming at eradication of tumor cells in minimal residual disease is an attractive experimental strategy for RCC, since long-term outcome of advanced disease remains poor. IL-7/CD80-cotransfected tumor cells are strongly immunogenic in mouse tumor models. Allogeneic vaccines may enhance immunogenicity and present a broad spectrum of tumor-associated antigens.

Methods: The human HLA-A0201+ RCC tumor cell line RCC26 was transfected with IL-7 and B7.1 (CD80), gene-modified vaccine cells were generated in our GMP facility. After approval by the committee for somatic gene therapy of the BÄK the study was registered in the German Clinical Trials Register (DRKS 00000294). 10 HLA-A0201+ patients with progressive, metastatic RCC were immunized repetitively over 22 weeks with $2.5-40 \times 10^{6}$ IL-7/CD80 cotransfected allogeneic tumor cells (RCC26-IL-7/CD80). Endpoints of the study were feasibility, safety, immunological and clinical responses. T cell responses against RCC-associated antigens were evaluated by g-IFN-ELISpot and cytokine bead array (CBA).

Regulatory T cells (Treg) were monitored in peripheral blood by PCR-based detection of a demethylated FoxP3 gene locus.

Results: Vaccination was feasible and safe; no grade $3 / 4$ toxicities were observed. $50 \%$ of the patients showed stable disease throughout the study; the median time to progression was 18 weeks. The median overall survival was 40 months, with 3 patients still alive in December 2009 (at a median follow-up of 41.5 months). However, the vaccine was not able to induce TH-1-polarized immune responses. A TH2 cytokine profile with increasing amounts of antigen-specific IL-10 secretion was observed in most of the patients with a detectable immune response. In some of these patients, increasing numbers of Tregs were detected. Interestingly, IFN-g secretion by patient lymphocytes upon antigen-specific and non-specific stimulation was substantially impaired, both before and during vaccination, as compared with healthy controls.

Conclusions: Vaccination with allogeneic RCC-26-IL-7/CD80 tumor cells is feasible and safe.
As measured by our immunomonitoring protocol, the vaccine was not able to induce TH-1-polarized immune responses. This is possibly due to pre-existing profound immunosuppression by the tumor preventing induction of anti-tumor immunity by the gene-modified vaccine. Depletion of regulatory cells before vaccination might be one strategy to circumvent this limitation.

Disclosure: Anne Flörcken: Honoraria: Bayer Schering Pharma Antonio Pezzutto: No conflict of interest disclosed.

\section{P915 \\ CD34+ progenitors as a relevant reservoir for JC virus - disproving a concept}

Warnke, C. ${ }^{1}$; Smolianov, V.1 ; Dehmel, T.1; Andree, M. ${ }^{1}$; Hengel, H. ${ }^{1}$; Zooren, F. ${ }^{1}$; Arendt, G. ${ }^{1}$; Wiendl, H. $^{2}$; Haas, R. $^{1}$; Hartung, H.-P. ${ }^{1}$; Adams, O. ${ }^{1}$; Kieseier, B.C. ${ }^{1}$

${ }^{1}$ Universität Düsseldorf, Düsseldorf, Germany, ${ }^{2}$ Universität Würzburg, Würzburg, Germany

Introduction: Progressive multifocal leukoencephalopathy (PML) is a known side effect of natalizumab (Tysabri®), an immunotherapeutic for patients with relapsing multiple sclerosis (MS) targeting the adhesion molecule VLA-4. The mechanism of JC-virus (JCV) reactivation by natalizumab remains elusive at present. However, one hypothesis discussed in the literature is the reactivation of JCV-infection by mobilization of JCV-infected bone marrow cells leading to JC-viremia and PML as a consequence of this specific therapy.

Methods: JCV-DNA and CMV-DNA were assessed in plasma, peripheral blood mononuclear cells (PBMCs), and magnetically isolated CD34+ cells of 67 MS patients while on treatment with natalizumab and six PML patients.

Results: Our data confirm previous results from our group and others that natalizumab induces an increase in CD34+ cell counts in the peripheral venous blood. This increase is independent of the number of treatments received and is maintained over the entire treatment period. In none of the studied patients, JCV- or CMV-DNA was observable in PBMCs or CD34+ hematopoietic progenitor cells. However, in two plasma sample, JCV-DNA was detectable. In one case, the patient developed clinical PML eight month thereafter.

Conclusions: Our present findings do not support the hypothesis that blocking VLA-4 mobilizes JCV-infected CD34+ cells out of the bone marrow into the peripheral venous blood. As such, alternative scenarios causing JCV reactivation in patients treated with natalizumab should be considered. Notably, one of the patients with positive JCV plasma findings in our cohort developed clinical PML eight month thereafter. Still, given the frequency of positive plasma findings in healthy individuals, it remains speculative, whether or not transient JC-viremia precedes development of clinical PML in general. This clearly warrants further investigation in large and diverse collectives applying standardized PCR-protocols.

Acknowledgments: The technical assistance of Tatjana Males is gratefully acknowledged. Part of this study was supported by the Forschungskommission of the Heinrich-Heine-University, Düsseldorf. This work was done partly as an effort within the German competence network of MS (KKNMS), funded by the German ministry of health and education ("Natalizumab pharmacovigilance study group").

Disclosure: Clemens Warnke: No conflict of interest disclosed.

Bernd Kieseier: Honoraria: B.C.K. has received honoraria for lecturing, travel expenses for attending meetings, and financial support for research from Bayer Health Care, Biogen Idec, Merck Serono, Novartis, Sanofi Aventis, and TEVA. 
P916

\section{Phase I/II trial of Zoledronic Acid plus Interleukin-2 in patients with solid and hematological malignancies}

Smetak, M. ${ }^{1}$; Kunzmann, V. ${ }^{2}$; Kimmel, B. ${ }^{2}$; Weigang-Koehler, K. ${ }^{1}$; Goebeler, M. ${ }^{2}$; Birkmann, J. ${ }^{1}$; Becker, J. ${ }^{3}$; Schmidt-Wolf, I. ${ }^{4}$; Einsele, H. $^{2}$; Wilhelm, M. ${ }^{1}$

${ }^{1}$ Institute of Medical Oncology and Hematology, Klinikum Nürnberg, Nürnberg, Germany, ${ }^{2}$ Medizinische Klinik und Poliklinik II and Early Clinical Trial Unit (ECTU), Julius Maximilians University, Würzburg, Germany, ${ }^{3}$ Department of Dermatology, Julius Maximilians University, Würzburg, Germany, ${ }^{4}$ Klinik und Poliklinik I, Rheinische Friedrich-Wilhelms-University, Bonn, Germany

Emerging evidence suggests that zoledronic acid (ZA) has antitumor properties including immunomodulatory effects through induction of $\gamma \delta \mathrm{T}$ cells. These effectors of the innate immune system just as NK cells are increasingly used for immunotherapy approaches. We and others have previously shown that aminobisphosphonates (ABP) are potent $\gamma \delta \mathrm{T}$ cell stimulatory compounds by inducing secretion of pro-inflammatory cytokines and cell-mediated cytotoxicity against several tumor cell lines in vitro. The results of two clinical trials revealed that an effective $\gamma \delta \mathrm{T}$ cell activation/expansion can also be induced in vivo by the combination of ABP and low dose interleukin-2 (IL-2). This multicenter Phase I/II study evaluated the safety, pharmacodynamics and antitumor activity of ZA in combination with low-dose IL-2 in adults with advanced malignancies. We examined the effect of adding low-dose IL-2 ( 2 x $10^{6} \mathrm{IU} / \mathrm{m} 2$ subcutaneously days 1 to 6 ) to ZA ( $4 \mathrm{mg}$ intravenously day 1 ) in 21 patients with different advanced malignancies (metastatic renal cell carcinoma; metastatic malignant melanoma and acute myeloid leukemia). The primary endpoints were safety and objective response rate. Blood samples were assessed for immune responses (pharmacodynamics) and serum levels of cytokines and VEGF. ZA in combination with low-dose IL-2 was well tolerated, with no grade 3-4 drug-related adverse events except for fever. The most common adverse effects (Grade 1-3) included fever and chills, postinfusional superficial phlebitis, injection site skin reaction, arthralgia and infections. 2 patients with chemotherapy refractory AML $(25 \%)$ achieved objective tumor responses (partial remission, IWG Response Criteria for AML). No objective responses were observed in both cohorts of solid tumors. Pharmacodynamic analyses showed significant in vivo activation and expansion of $\gamma \delta \mathrm{T}$ cells ( $\mathrm{V} \gamma 9 \mathrm{~V} \delta 2$ subset) accompanied by production of IFN- $\gamma$ in all evaluable patients. Interestingly, high pretreatment serum VEGF levels and unexpected increase of VEGF induced by ZA plus lowdose IL-2 were correlated with lack of clinical response. Conclusion: Although zoledronic acid plus IL-2 appeared well tolerated and induced significant immunologic responses, objective clinical response rate is low und restricted to hematological malignancies. VEGF serum level was identified as a new biomarker counteracting potential $\gamma \delta \mathrm{T}$ cell-mediated antitumor activity in vivo and should be validated in upcoming clinical trials.

Disclosure: Manfred Smetak: No conflict of interest disclosed. Martin Wilhelm: Advisory Role: Novartis, Sandoz

\section{P917}

\section{HMGB1 serum levels in patients with hematologic malignancies before and following chemotherapy}

Jost, K. ${ }^{1}$; Killian, D. ${ }^{1}$; Sekora, A. ${ }^{1}$; Escobar, H. ${ }^{2}$; Nolte, I. ${ }^{2}$; Kahl, C. ${ }^{1}$; Freund, M. ${ }^{1}$; Junghanss, C. ${ }^{1}$

${ }^{1}$ Zentrum für Innere Medizin, Uniklinik Rostock, Hämatologie, Onkologie und Palliativmedizin, Rostock, Germany, ${ }^{2}$ Tierärztliche Hochschule Hannover, Klinik für Kleintiere, Hannover, Germany

Introduction: The efficacy of anticancer treatment is associated with its direct ability to inhibit tumor cell proliferation. However recent data shows that an immunoadjuvant pathway triggered by the release of endogenous danger signals by the dying tumor cell also contributes to the success of cyctotoxic therapies. High mobility group box 1 (HMGB1) is one of these alarmins, a nuclear protein that is released during infection and tissue damage to mediate inflammation and facilitate optimal presentation of antigens from dying tumor cells. We hypothesized that HMGB1 levels are altered following chemotherapy. The aim of this study was to evaluate HMGB1 serum levels in patients with hematologic malignancies before and after chemotherapy.
Methods: Samples of 25 patients (mean age 57 years (range 28-70)) with hematologic malignancies (multiple myeloma $\mathrm{n}=20$, Non Hodgin lymphoma $\mathrm{n}=3$, acute myeloid leukemia $=2$ ) were investigated. Patients with multiple myeloma were treated with cyclophosphamide $(n=1)$ or high dose melphalan followed by autologous stem cell transplantation $(n=19) .5$ patients received an allogenic stem cell transplantation following different conditioning regimens (reduced intensity protocols $n=4$, myeloablative $n=1$ ). HMGB1 serum levels were measured by an enzyme-linked immunosorbent assay (ELISA) before the first chemotherapy agent was given (day 0) and on the 3 following mornings (day 1 to 3 ).

Results: The mean HMGB1 levels on days $0,1,2$, and 3 were $5.46 \mathrm{ng} / \mathrm{ml}$ (range 0.1 - 13.6), $10.17 \mathrm{ng} / \mathrm{ml}$ (range $0.4-39.2, \mathrm{n}=23$ ), $6.42 \mathrm{ng} / \mathrm{ml}$ (range 0.9 - 22.5) and $7.36 \mathrm{ng} / \mathrm{ml}$ (range 0-27.5) respectively. In the multiple myeloma patients who received high dose melphalan the data were $6.68 \mathrm{ng} / \mathrm{ml}(0.1$ 13.6) on day $0,10.46 \mathrm{ng} / \mathrm{ml}(0.4-29.0)$ on day $1,7.28 \mathrm{ng} / \mathrm{ml}(0.9-22.5)$ on day 2 and $8.21 \mathrm{ng} / \mathrm{ml}(0-27.5)$ on day 3. Although mean HMGB-1 levels were higher on day 1 there was great interindividual heterogenicity. No correlation could be shown between HMGB-1 levels and C-reactive protein or leucocyte count. There was no relationship between tumor remission status and HMGB-1 levels before or after chemotherapy although most patients $(n=21)$ were at least in partial remission due to previous chemotherapies.

Conclusions: Our preliminary data suggests that HMGB1 serum levels are not significantly altered following chemotherapy administration in hematological patients that have limited tumor burden.

Disclosure: No conflict of interest disclosed.

\section{P918}

Investigation of five commercially intravenous immunoglobulines in immunocompromised haematological patients regarding their ability to inhibit CMV infection, transmission and to enhance NK-cellmediated lysis

Ganepola, S. ${ }^{1,2}$; Frenzel, K. ${ }^{3}$; Krüger, D.H. ${ }^{3}$; Thiel, E. ${ }^{1}$; Hofmann, J. $^{3}$; Uharek, L. ${ }^{1}$

${ }^{1}$ II. Medical Clinic, Charite University Medicine, Berlin, Department of Hematology, Oncology and Transplantation Medicine, Berlin, Germany, ${ }^{2}$ present address: Asklepios Klinik Altona, II. Medical Department, Hematology, Oncology and Transplantation Medicine, Hamburg, Germany, ${ }^{3}$ Institut of Medical Virology, Charite University Medicine, Berlin, Germany

Primary infection and reactivation of human cytomegalovirus (HCMV) is one of the major problems in immunocompromised patients, especially after allogeneic stem cell transplantation, frequently resulting in life threatening diseases. Intravenous (hyper)immunoglobulins (Ig) can be administered for prophylaxis against HCMV infection. However, the clinical impact is a subject of controversy today and the benefit especially for stem cell transplant recipients remains uncertain, as several studies revealed contradictory results. To evaluate the influence of different Ig products, we performed a qualitative and quantitative comparison of five commercially available intravenous immunoglobulins concerning their ability to neutralize circulating virus, to inhibit cell associated transmission and to opsonize infected cells for NK cell mediated lysis.

The neutralizing activity (NA) was tested against the HCMV laboratory strain AD169 and four clinical isolates. To approach the situation in vivo, epithelial cells infected with the HCMV strain TB40 were used. As expected, a CMV specific hyperimmunoglobulin showed the highest NA regardless of the virus or cell type. Within the different clinical strains we observed differences in the $50 \%$ inhibition dose over a 3-log range. Surprisingly, no correlation between the neutralizing antibody titre and the ability to inhibit cell to cell spread or cell-cell fusion was found. Cytotoxicity assays using CMV infected fibroblasts and NK cells from a CMV seronegative donor suggest an additional impact of some but not all immunoglobulins by enhancing the NK-cell mediated lysis by antibody dependent cellular cytotoxicity.

In summary, to characterize the effect of an immunoglobulin on viral replication all possible mechanisms of action must be taken into account and individual testing could be performed finding the most effective Ig.

Disclosure: No conflict of interest disclosed. 
P919

\section{Donor transmission of lung cancer to a lung graft recipient: A case report}

von Boehmer, L. ${ }^{1}$; Draenert, A. ${ }^{2}$; Boehler, A. ${ }^{3}$; Soltermann, A. ${ }^{4}$; Thiesler, T. ${ }^{4}$; Schäfer, N. ${ }^{1}$; Stahel, R. ${ }^{1}$; van den Broek, M. ${ }^{1}$; Weder, W. ${ }^{2}$; Knuth, A. ${ }^{1}$

${ }^{1}$ UniversitätsSpital Zürich, Onkologie, Zürich, Switzerland,

${ }^{2}$ Universitätsspital Zürich, Thoraxchirurgie, Zürich, Switzerland,

${ }^{3}$ Universitätsspital Zürich, Pneumologie, Zürich, Switzerland,

${ }^{4}$ Universitätsspital Zürich, Pathologie, Zürich, Switzerland

Introduction: There are multiple lines of evidence for immunosurveillance and immunoediting of cancers. During the past 20 years, three independent cases were described, where after bilateral lung transplantion, patients developed donor-derived tumors, although donors had no clinical history of malignancy. In such cases, the tumor or a premalignant lesion presumably was held 'in check' by the donor immune system, but grew out after transplantation into an immunosuppressed host. We present the clinical history of a 61-yr-old female, who suffered end-stage idiopathic pulmonary fibrosis with characteristic features of usual interstitial pneumonia (UIP). Bilateral lung transplantation was performed with adequate immunosuppression to prevent graft rejection. The transplanted patient developed a primary large cell carcinoma of the lung 10 months after lung transplantation.

Methods: SSP Genovision analysis of Tumor DNA was performed to investigate the origin of the tumor (recipient or donor?). Immunohistochemistry was used for immune infiltrate analysis in the tumor tissue. And FACS was performed on peripheral blood mononuclear cells (PBMC) of the recipient under immunosuppression.

Results: HLA-DR 11 (only expressed in the donor) and HLA-DR 14 (only expressed in the recipient) was analyzed. Only HLA-DR 11 was positive, demonstrating that the graft carcinoma was of donor origin. Although a dense cellular infiltrate in the tumor was seen, no immunohistochemical staining for granzyme and perforin was detected revealing a lack of cytotoxic effector cells under continued immunosuppression. PBMC analysis compared to healthy controls showed a lack of B and NK cells as well as memory T cells. In line with this finding, we found less $\mathrm{IL}-2$ production after unspecific stimulation (IL-2 is produced mainly by memory T cells).

Conclusions: We describe here a patient, developing lung cancer of donor origin under immunosuppression after bilateral lung graft as treatment of endstage idiopathic pulmonary fibrosis. This case report provides further evidence for immunosurveillance of cancer in vivo.

Disclosure: No conflict of interest disclosed.

Wissenschaftliches Symposium
Damage to self

\section{V927}

Netting neutrophils: NETs in immunodefiency and autoimmunity

\section{Zychlinsky, A. $^{1}$}

${ }^{1}$ Max-Planck-Institut für Infektionsbiologie, Berlin, Germany

Neutrophils are one of the first lines of defence of the immune system against microbes. These cells kill microorganisms effectively by phagocytosis. We identified a new killing mechanism: stimulated neutrophils produce extracellular structures, called neutrophil extracellular traps (NETs) that capture and kill microorganisms. After stimulation, neutrophils produce radical oxygen species (ROS) that trigger irreversible morphological changes like nuclear membrane disintegration, chromatin decondensation and loss of cytoplasmic organelles. Later, chromatin mixes with granular components intracellularly and when the plasma membrane is disrupted the NETs are released. This process is distinct from apoptosis and necrosis. We now identified through a proteomic approach the complete NET composition and identified calgranulin as its main antifungal component. This novel ROS dependent death program allows neutrophils to fulfill their antimicrobial function even beyond their life span.

Disclosure: No conflict of interest disclosed.
V928

Mast cell proliferation and malignant mast cell disorders

Valent, $P .^{1,2}$

${ }^{1}$ Medical University of Vienna, Department of Internal Medicine I, Division of Hematology, Vienna, Austria, ${ }^{2}$ Ludwig Boltzmann Cluster Oncology, Vienna, Austria

Mastocytosis is a myeloid neoplasm characterized by abnormal accumulation and sometimes activation of tissue mast cells (MC) in various organs. Clinical systems result from effects of mast cell derived mediators and/or pathologic infiltration of various organs by $\mathrm{MC}$ with consecutive organ destruction, which is seen primarily in advanced systemic mastocytosis (SM). In a substantial number of patients the skin is affected. Mastocytosis can be divided into cutaneous mastocytosis (CM) and SM as well as the rare localized MC tumors, i.e. mastocytomas and MC sarcoma. CM is usually diagnosed in (early) childhood. By contrast, most adult patients are suffering from SM. The WHO classification discriminates between indolent SM (ISM), SM with an associated clonal hematologic non-MC-lineage disease (SM-AHNMD), aggressive SM (ASM), and mast cell leukemia (MCL). Organ systems frequently involved in SM are the bone marrow, skin, liver, and the gastrointestinal tract. In a vast majority of all patients with SM, the KIT mutation D816V is detectable, independent of the category of SM. Since in all SM patients the course of disease ranges from completely asymptomatic with normal life expectancy (ISM) to highly aggressive with short $(<1 \mathrm{y})$ survival times $(\mathrm{MCL})$, other (additional) oncogenic factors are considered to lead to transformation in advanced SM. In these patients but not in patients with ISM, MC express CD30 and are triggered by additional KIT-independent signalling pathways involving Btk and/ or Lyn. Other factors, such as a co-existing allergy, may lead to MC activation with consecutive anaphylactic reactions which can be severe or even lifethreatening in SM. Treatment of ISM is usually focusing on symptom relieve by histamine receptor antagonists and other supportive therapy. However, in aggressive and leukemic variants, cytoreductive and targeted drugs need to be applied. Unfortunately, the prognosis in these patients remains poor even when treated with novel KIT-targeting agents, polychemotherapy, or stem cell transplantation.

Disclosure: Peter Valent: Financing of Scientific Research: Forschungsunterstützung: Merck-Serono, Novartis, Domantis; Other Financial Relationships: Reisekosten: Novartis

\section{Freie Vorträge Multiples Myelom experimentell 2 \\ v935 \\ Inhibition of HIF1A signaling by a novel class of sulfonanilides for targeted treatment of multiple myeloma}

Hose, D. ${ }^{1,2}$; Seckinger, A. ${ }^{1}$; Goldschmidt, H. ${ }^{1,2}$; Meißner, T.'; Rebacz, B. ${ }^{3,4}$; Moreaux, J. ${ }^{5,6}$; Benes, V. ${ }^{3}$; Neben, K. ${ }^{1}$; Hillengass, J. ${ }^{1}$; Bertsch, U. ${ }^{1}$; Jauch, A. ${ }^{7}$; Rossi, J.F. 5,6; Möhler, T. ${ }^{1}$; Zimmermann, J. ${ }^{3}$; von Kalle, C. ${ }^{2}$; Lewis, J. ${ }^{3,4}$; Klein, B. ${ }^{5,6}$; Schultes, C. ${ }^{3,4}$

${ }^{1}$ Universitätsklinikum Heidelberg, Medizinische Klinik V, Heidelberg, Germany, ${ }^{2}$ Nationales Centrum für Tumorerkrankungen, Heidelberg, Germany, ${ }^{3}$ European Molecular Biology Laboratory, Heidelberg, Germany, ${ }^{4}$ ELARA Pharmaceuticals, Heidelberg, Germany, ${ }^{5} \mathrm{CHU}$ Montpellier, Montpellier, France, ${ }^{6}$ INSERM U847, Montpellier, France, ${ }^{7}$ Universitätsklinikum Heidelberg, Institut für Humangenetik, Heidelberg, Germany

Background: Molecular profiling of multiple myeloma allows the identification of novel targets, including HIF1A, and evaluation of their expression within large cohorts of patients. We report here the expression of HIF1A and the preclinical testing of 4 members of a novel class of sulfonanilide HIF1A signaling inhibitors.

Patients and methods: Expression of HIF1A was assessed using Affymetrix DNA-microarrays in 329 samples of CD138-purified myeloma cells from previously untreated patients. Chromosomal aberrations were assessed by comprehensive iFISH, proliferation of primary myeloma cells $(n=67)$ was determined by propidium iodide staining. The effect of the novel HIF1A signaling inhibitors ELR510490, ELR510454, ELR510444 and ELR105813 on 
the proliferation of 12 myeloma cell lines and the first three on the survival of 5 primary myeloma cell-samples was tested, and their ability to inhibit HIF1A signaling was examined. Studies were also conducted to determine in vitro stability as well as single-dose PK parameters and maximum tolerated dose levels after dosing in mice.

Results: We found (i) HIF1A to be expressed by $95.4 \%$ of myeloma cell samples. (ii) HIF1A expression shows a weak but significant correlation ( $\mathrm{r}=0.3, P<0.001)$ with a gene expression based proliferation index. (iii) Of the chromosomal aberrations tested, myeloma cells of patients with a translocation $\mathrm{t}(4,14)$ show a significantly higher expression of HIF1A $(P<0.001)$ vs. patients without. Myeloma cells of hyperdiploid patients show a significantly lower expression of HIF1A ( $P=0.02)$ vs. non hyperdiploid. (iii) HIF1A expression does not show a correlation with event-free or overall survival. (iv) The compounds completely inhibit proliferation of all tested cell lines at nM concentrations and (v) are active on all primary myeloma cell-samples. (vi) The compounds show a pronounced effect on the HIF1A signaling pathway at $\mathrm{EC}_{50} \mathrm{~s}$ of $1-25 \mathrm{nM}$. (vii) Pre-clinical pharmacology data in mice indicate favorable absorption, distribution, metabolism and excretion profiles as well as exposure levels upon dosing at well-tolerated levels that are significantly above the in vitro $\mathrm{EC}_{50}$ in all the cell lines tested.

Conclusion: HIF1A is expressed in almost all primary myeloma cells. The novel HIF1A signaling inhibitors tested are very active on myeloma cell lines as well as primary myeloma cells and show favorable in vivo profiles. This class of compounds thus represents a promising weapon against multiple myeloma

Disclosure: Dirk Hose: No conflict of interest disclosed.

Christoph Schultes: Employment or Leadership Position: ELARA Pharmaceuticals

\section{V936}

The paraproteins of patients with familial MGUS / multiple myeloma (MM) target family-specific antigens: paratarg-7 and paratarg-8

Grass, S. ${ }^{1}$; Preuss, K.-D. ${ }^{1}$; Wikowicz, A. ${ }^{1}$; Thomé, S. ${ }^{2}$; Weisenburger, D.D. ${ }^{3}$; Witt, V. ${ }^{4}$ : Lynch, J. ${ }^{2}$ Pfreundschuh, M. ${ }^{1}$; Lynch, $\mathrm{H}^{2}{ }^{2}$

${ }^{1}$ Unikliniken des Saarlandes, Klinik für Innere Medizin I, Homburg (Saar), Germany, ${ }^{2}$ Creighton University School of Medicine, Department of Preventive Medicine and Public Health, Omaha, United States, ${ }^{3}$ Nebraska Medical Center, Omaha, United States, ${ }^{4}$ Newberry College, Newberry, United States

Background: Antigenic targets of paraproteins in monoclonal gammopathy of undetermined significance (MGUS) and multiple Myeloma (MM) might play a role in the pathogenesis of these neoplasms by chronic antigenic stimulation. However, very few antigens have been identified, of which most were specific for one individual paraprotein only. In contrast, we recently described paratarg-7 (P-7), a protein of unknown function which is expressed in all human tissues as the target of $15 \%$ of $\operatorname{IgA}$ and $\operatorname{IgG}$ paraproteins in MGUS and MM (Grass et al. Lancet Oncology 2009; 10: 950-956). MGUS/MM patients with P-7 specific paraproteins all carry a hyperphosphorylated variant of P-7 (pP-7), which is inherited in a dominant fashion, explaining $\mathrm{pP} 7$-posistive cases of familial MGUS/MM. pP-7 is the first molecularly defined autosomaldominant risk factor for MGUS/MM and is associated with the highest odds ratio (7.9) of all risk factors for MGUS/MM known to date. Since not all cases of familial MGUS/MM have a P-7 specific paraprotein, we set out to identify the antigenic targets of paraproteins in cases of familial MGUS/MM that are not carriers of $\mathrm{pP}-7$.

Methods: The sera of 28 members of a family with familial MGUS/MM (Lynch et al. N Engl J Med 2008;359:152-157) were tested for antibody reactivity against antigens represented in a fetal brain derived protein macroarray using a modified SEREX approach (Preuss et al. International J. Cancer 2009; 125, 656-661).

Results: Sera from 28 family members were analyzed. The sera of all 6 family members affected by MGUS $(n=3)$ and MM $(n=3)$ reacted with paratarg-8, which is encoded by the ATG13 gene, a member of the ,,autophagy regulatory complex" family of genes. The titers ranged from $1: 10^{7}$ to $1: 10^{8}$. No antibody reactivity was detected in the sera of non-affected family members nor in the paraproteins from $>300$ other patients tested.
Conclusions: The paraproteins of members affected by familial MGUS/MM are directed against family-specific antigenic targets, suggesting that the genetic background shared by these patients entertains a chronic auto-immune response. Paratarg-7 and paratarg- 8 are the first family-specific antigens that have been molecularly defined to date. Whether the antigen/paraprotein interaction is an epiphenomeon or invovled in the pathogenesis of familial cases of MGUS/MM can now be addressed using specific molecular tools.

Disclosure: No conflict of interest disclosed.

\section{V937}

Association of hyperphosphorylated paratarg-7, the first dominantly inherited risk factor in hematological neoplasia with IgM-MGUS and Waldenstrom's macroglobulinemia (WM)

Grass, S. ; Preuss, K.-D. ${ }^{1}$; Wikowicz, A. ${ }^{1}$; Terpos, E. ${ }^{2}$; Ziepert, M. ${ }^{3}$; Ahlgrimm, M. ${ }^{1}$; Fadle, N. ${ }^{1}$; Regitz, E. ${ }^{1}$; Murawski, N. ${ }^{1}$; Dimopoulos, M.A. ${ }^{2}$; Treon, S.P. ${ }^{4}$; Hunter, Z.R. ${ }^{4}$; Pfreundschuh, M. ${ }^{1}$ ${ }^{1}$ Saarland University Medical School, Dep. Internal Medicine I, JoseCarreras-Center for Immuno- and Gene Therapy, Homburg/Saar, Germany, ${ }^{2}$ University of Athens School of Medicine, Department of Clinical Therapeutics, Athens, Greece, ${ }^{3}$ IMISE, Leipzig, Germany, ${ }^{4}$ Bing Center for Waldenstrom's Macroglobulinemia, Dana Farber Cancer Institute, Harvard Medical School, Boston, United States

Background: We recently described paratarg-7, a protein of unknown function which is expressed in all human tissues as the target of $15 \%$ of $\operatorname{IgA}$ and IgG paraproteins in MGUS and MM.

Methods: To determine if and how frequently paratarg-7 functions as the antigenic target of the IgM paraproteins in MGUS/WM, sera from patients with IgM-MGUS/WM were tested for reactivity with recombinant paratarg-7 by ELISA. Lysates of peripheral blood from patients and controls were tested by gel electrophoresis and isoelectric focusing before and after phosphatase treatment.

Results: The paraproteins of 18 (9 WM and 9 IgM-MGUS) of 161 (11\%) patients reacted specifically with paratarg-7, proving paratarg-7 as the first antigen identified as a paraprotein target in a significant proportion of patients with IgM-MGUS/WM. 2D-gelelectrophoresis, isoelectric focusing and phosphatase treatment revealed that paratarg-7 was hyperphosphorylated in all patients with an anti-paratarg-7 specific IgM-paraprotein tested. In contrast, only 4 of 200 (2\%) healthy blood donors were carriers of hyperphosphorylated paratarg-7. Thus, carriers of hyperphosphorylated paratarg-7 have a significantly increased risk (odds ratio $=6.5 ; 95 \%-\mathrm{CI}: 2.1-19.6 ; \mathrm{p}=0.001$ ) for developing IgM-MGUS/MW. Moreover, family analyses of relatives of IgM-MGUS/ WM patients with an anti-paratarg-7 specific paraprotein revealed that the hyperphosphorylated state of this protein is inherited as a dominant trait.

Conclusions: Hyperphosphorylated paratarg-7 is a highly significant risk factor for MGUS, WM and MM, with the highest odds ratio of any risk factor reported to date for these diseases. The carrier state of hyperphosphorylated paratarg-7 explains cases with familial MGUS, MM and WM and enables the identification of family members of patients at increased risk for MGUS/WM/ MM. Dominant inheritance and the identification of familial cases with MGUS/MM/WM and hyperphosphorylated paratarg-7 carrier state facilitate genome-wide screens for the identification of the SNP responsible for hyperphosphorylation of this molecule.

Disclosure: No conflict of interest disclosed 
V938

Proliferation and activation patterns of naïve, memory and regulatory $\mathrm{T}$ cells in patients with multiple myeloma during thalidomide, interferon- $\alpha$ and bortezomib maintenance therapy

Hundemer, M. ${ }^{1}$; Herth, I. ${ }^{1}$; Meissner, T. ${ }^{1}$; Hose, D. ${ }^{1}$; Ho, A. ${ }^{1}$; Neben, K. ${ }^{1}$; Goldschmidt, H. ${ }^{1}$; Witzens-Harig, M. ${ }^{1}$

${ }^{1}$ Universitätsklinik Heidelberg, Heidelberg, Germany

Introduction: In patients with Multiple myeloma, maintenance therapy after high-dose chemotherapy and autologous stem cell transplantation is performed with the aim to prolong remission duration and survival. In this prospective study we have analysed the implication of the various types of maintenance therapy on the patients T-cell pattern and activation status.

Patients and methods: T-cells from 63 patients in clinical remission were analyzed. Eighteen patients were treated with IFN- $\alpha, 22$ with thalidomide, 7 with bortezomib and 16 patients received no maintenance therapy. Activated and nonactivated T-cells were analyzed by intracytoplasmatic staining, flow cytometry and ELISA.

Results: Patients treated with IFN- $\alpha$ showed a high rate of naïve T-cells, while in the thalidomide-group a high rate of effector memory T-cells were observed. The rate of IFN- $\gamma$-producing $\mathrm{CD}^{+}{ }^{+} \mathrm{T}$-cells was significant higher in the thalidomide-group than in the IFN- $\alpha$-group after activation $(\mathrm{p}=0.02)$. On the basis of the cytoplasmatic staining of Foxp3 there was a trend to a higher amount of regulatory T-cells in the thalidomide-group compared to the control-group $(\mathrm{p}=0.08)$. Analysis of IFN-y-secretion by ELISA, an increases IFN- $\boldsymbol{\gamma}$-secretion could be demonstrated in all groups after activation (control-group: $\mathrm{p}=0.002$, IFN- $\alpha$-group: $\mathrm{p}<0.001$, thalidomide-group: $\mathrm{p}<0.001$, bortezomib-group: $\mathrm{p}=0.01$ ), furthermore in all groups despite the bortezomib-group an increase of the granzyme B-production can be observed (control-group: $\mathrm{p}=0.003$, IFN$\alpha$-group: $p=0.03$, thalidomide-group: $\mathrm{p}<0.001)$. Regarding the activated state of the T-cells the production of IFN- $\gamma$, perforine and granzyme B was significant higher in the thalidomide-group than in the IFN- $\alpha$-group (IFN- $\gamma$ : $p=0.05$, perforine: $\mathrm{p}=0.02$, granzyme $\mathrm{B}: \mathrm{p}=0.04$ ). Furthermore the nonactivated and the activated T-cells of the patients treated with thalidomide showed a significant higher production of granzyme B than the T-cells of the control-group ( $\mathrm{p}=0.0003$ and $\mathrm{p}=0.006$ ).

Conclusion: During maintenance therapy, thalidomide promotes maturation and proliferation of effector memory T-cells and regulatory T-cells, while IFN- $\alpha$ treatment increases the number of naïve T-cells and subsequently, the T-cell activation in the thalidomide-group was significantly higher than in the IFN- $\alpha$-group. These results might have impact on the development of novel immunomodulating therapy

First and second author contributed equally

Disclosure: No conflict of interest disclosed.

\section{V939}

\section{Generation and preclinical evaluation of Fc-optimized RANK-Ig fusion proteins for neutralization of soluble RANKL and targeting of Myeloma and CLL cells for NK reactivity}

Scheible, C. ${ }^{1}$; Schmiedel, B.J. ${ }^{1}$; Baessler, T.1; Wende, C.M. ${ }^{1}$; Wirths, S. ${ }^{1}$; Azuma, M. ${ }^{2}$; Schneider, P. ${ }^{3}$; Grosse-Hovest, L. ${ }^{4}$; Kanz, L. ${ }^{1}$; Salih, H.R. ${ }^{1}$

${ }^{1}$ Eberhard Karls University, Department of Hematology and Oncology, Tübingen, Germany, ${ }^{2}$ Tokyo Medical and Dental University, Department of Molecular Immunology, Tokyo, Japan, ${ }^{3}$ University of Lausanne, Department of Biochemistry, Epalinges, Switzerland, ${ }^{4}$ Eberhard Karls University, Department of Immunology, Tübingen, Germany

Introduction: RANKL and its receptors RANK and OPG are key regulators of bone remodelling. Multiple myeloma (MM) disrupts the balance within this molecule system towards osteoclastogenesis and bone destruction. Moreover, CLL cells were found to express RANKL. Neutralization of soluble RANKL (sRANKL) using Denosumab is presently being evaluated for treatment of both non-malignant and malignant osteolysis. Here we studied the possibility of targeting RANKL-expressing malignant hematopoietic cells for NK cells, which play an important role in immunesurveillance due to their ability to directly lyse cancer cells and to mediate antibody-dependent cellular cytotoxicity (ADCC) against tumor targets.

Methods: RANKL expression and release was analyzed by FACS, RT-PCR and ELISA (PeproTech, Germany). RANK-Fc fusion proteins with distinct effector functions in their Fc portion were generated as previously described (Lazar et al., 2006; Armour et al., 1999). Effects of RANKL neutralization and targeting were analyzed in osteoclast formation assays, cytotoxicity assays and ELISA.

Results: Primary MM and CLL ( $\mathrm{n}=9$ of 10 and all of 40, respectively) cells express and release substantial levels of RANKL, while no release was observed with PBMC of healthy donors. Compared to wildtype RANK-Fc (RANK-Fc-wt), our mutants (S239D/I332E and E233P/L234V/L235A/ $\Delta$ G236/A327G/A330S) displayed highly enhanced (RANK-Fc-ADCC) and abrogated (RANK-Fc-KO) affinity, respectively, to the NK cell FcgRIIIa but comparable capacity to neutralize RANKL in binding competition and osteoclast formation assays. In cocultures of NK cells with RANKL transfectants and RANKL-negative controls, addition of RANK-Fc-KO did not alter NK reactivity. Presence of RANK-Fc-wt caused minor effects, while RANK-FcADCC dramatically enhanced NK cytotoxicity and cytokine production against RANKL-expressing, but not RANKL-negative targets. This confirmed that the RANK-Fc-ADCC specifically induced NK reactivity against RANKLexpressing malignant cells. Moreover, treatment with RANK-Fc-ADCC also significantly increased anti-tumor reactivity of both allogenic and autologous NK cells against RANKL-positive primary malignant hematopoietic cells Conclusions: The Fc-engineered RANK-Fc-ADCC fusion protein may both neutralize detrimental effects of sRANKL and target NK anti-tumor reactivity to RANKL-expressing malignant cells. It may thus constitute an attractive immunotherapeutic means for treatment of hematopoietic malignancies.

Disclosure: No conflict of interest disclosed.

\section{V940}

\section{Oncogenic Ras and constitutively activated Akt promote multiple myeloma cell survival independently of each other}

\section{Steinbrunn, T. ${ }^{1}$; Stühmer, T. ${ }^{1}$; Chatterjee, M. ${ }^{1}$; Gattenlöhner, S. ${ }^{2}$;} Einsele, H. ; Bargou, R.

${ }^{1}$ Universitätsklinikum Würzburg, Medizinische Klinik und Poliklinik II, Würzburg, Germany, ${ }^{2}$ Universitätsklinikum Würzburg, Pathologisches Institut, Würzburg, Germany

Introduction: Oncogenic Ras is a potential activator of survival pathways in numerous cancer entities and Ras mutations are frequent in multiple myeloma (MM). About half of primary MM samples depend on constitutively active Akt, which renders these samples susceptible to inhibition of Akt in vitro. We were therefore interested to analyze the role of oncogenic Ras for survival of MM cells and its importance for Akt signaling.

Methods: 33 different primary MM samples were treated with Akt inhibitor Akti-1,2 and their relative viability was determined via flow cytometry. To correlate the results with the respective Ras status, the samples were screened for K- and N-Ras mutations by RT-PCR. Corresponding bone marrow biopsies were screened for presence of activated Akt by immunohistochemical CD138/ phospho-Akt double staining. Using siRNA-mediated knockdown, Ras wildtype and mutated MM cell lines were depleted of K- or N-Ras isoforms and the effects on viability and Akt-signaling were analyzed. To characterize the isoform specificity of the siRNAs, their efficacy was tested with ectopically expressed HA-tagged Ras proteins.

Results: K- or N-Ras mutations were found in $42.4 \%$ of primary MM samples, whereas $44 \%$ were classified as sensitive to inhibition of Akt. However these properties were not correlated, as Ras mutations were evenly distributed among the Akt-dependent and the Akt-independent MM subgroups. We therefore assume that constitutive activation of Akt does not rely on oncogenic Ras. Isoform-specific Ras knockdown in MM cell lines led to cell death specifically in the N-Ras mutated INA-6 and JJN-3 cells upon depletion of N-Ras, while K-Ras mutated MM.1S cells were more sensitive to knockdown of K-Ras. Neither of these siRNAs affected Ras wildtype U266 and AMO-1 cells. Of note, Ras knockdown did not reduce the level of phosphorylated Akt, which again suggests that constitutive Akt activation is not a consequence of onco- 
genic Ras. Combined knockdown of K-Ras and Akt1 in MM.1S cells enhanced the level of cell death significantly.

Conclusions: Although not mediated via activation of the Akt-pathway, oncogenic Ras still promotes survival of MM cell lines. Therefore, combined inhibition of both of these targets might enhance anti-myeloma effects also in clinical settings, providing an attractive therapeutic strategy for MM treatment.

Disclosure: No conflict of interest disclosed.

\section{Arbeitskreis Sitzung \\ Stammzellen für die Regenerative Medizin: Hype or Hope? \\ V943 \\ Gene-modified stem cells: Rational approaches to prevent off-target effects}

Baum, C. ${ }^{1}$; Modlich, U. ${ }^{1}$; Bode, J. ${ }^{1}$; Schiedlmeier, B. ${ }^{1}$; Meyer, J. Li, Z. ${ }^{1}$; Cathomen, T. ${ }^{1}$; Schambach, A. ${ }^{1}$

${ }^{1}$ Medizinische Hochschule Hannover, Experimentelle Hämatologie, Hannover, Germany

Introduction: An increasing number of clinical studies indicates the potentia of gene-modified, autologous hematopoietic stem cells to correct genetic disorders of blood cell formation or function. Major progress in the understanding of vector-host interactions has opened rational approaches to increase the therapeutic index with improved technologies for gene delivery. Furthermore, induced pluripotent stem cells have been introduced as an innovative platform for cell-based therapy.

Methods: Work in our department focuses on the analysis of the life cycle of various retroviruses (HIV-1, MLV, ASLV) as a basis for vector development, improvements of technologies for targeted genome engineering, and murine models and cell culture systems to create a new approach to "transgene toxicology".

Results: Our experiments identified a hierarchy of factors that dictate the emergence of insertional mutants: the "stemness" of the target cells, the presence of a strong enhancer-promoter in the integrated transgene, the insertion pattern of the vectors, and systemic homeostatic mechanisms. We revealed the transforming capacity of insertional activation of cellular proto-oncogenes such as Evil or Prdm16, and developed integrating gene vectors that reduce the risk of insertional transformation by altering the insertion pattern and the expression cassette of the transgene. Proof-of principle was obtained for correction of a signaling defect of hematopoietic stem cells by transcriptionally targeted lentiviral vectors. Towards the aim of generating de novo hematopoiesis derived from induced pluripotent cells, we have developed a genetically reversible lentivirus-based system that induces pluripotency at high efficiency, with Flp-recombinase mediated excision of "all-in-one" self-silencing reprogramming vectors. Robust hematopoietic differentiation of pluripotent murine cells was achieved in a multistep culture system supported by regulated expression of the transcription factor, HOXB4.

Conclusions: Studying mechanisms of interactions of gene vectors, targe cells and systemic host factors creates a rich resource of novel approaches in the development of stem cell-based therapies. Proactive analysis of potential off-target effects is crucial for a rational development of the field.

Disclosure: Christopher Baum: Employment or Leadership Position Angestellt bei der Medizinischen Hochschule Hannover, Patentanmelder für alpharetrovirale Gentransfervektoren; Stock Ownership: Anteilseigner Vision7 $\mathrm{GmbH}$; Financing of Scientific Research: BMBF, DFG, EU, FDA; Expert Testimony: EU, DFG, Europäische nationale Forschungsförderstellen und Stiftungen, NIH, Wiss. Zeitschriften

Axel Schambach: Employment or Leadership Position: Angestellt bei der Medizinischen Hochschule Hannover, Patentanmelder für alpharetrovirale Gentransfervektoren; Financing of Scientific Research: BMBF; Expert Testimony: Wiss. Zeitschriften

\section{Arbeitskreis Sitzung Neues zur Studienlandschaft der Nicht-malignen Hämatologie \\ V945 \\ Clinical complement inhibition by the anti-C5 antibody Eculizumab: Update on clinical trials}

Röth, A. $^{1}$

${ }^{1}$ Universitätsklinikum Essen, Klinik für Hämatologie, Essen, Germany

The complement system is an important part of the innate immunity and provides critical immunoprotective and immunoregulatory functions. Uncontrolled complement activation, however, can cause or worsen disease. The novel humanized monoclonal anti-C5 antibody eculizumab is able to block the proinflammatory and cytolytic effects of the terminal complement activation. In paroxysmal nocturnal hemoglobinuria (PNN) the affected cells are deficient in all GPI-anchored surface proteins due to mutations of the PIG-A gene including the complement regulators CD55 and CD59. Therefore, PNH RBCs are highly vulnerable to activated complement with the formation of the membran attack complex (MAC) resulting in the chronic intravascular hemolysis. Eculizumab blocks the MAC formation along with the intravascular hemolysis leading to a marked, decrease of anemia, fatigue, transfusion requirements, renal impairment, pulmonary hypertension, the risk of severe thromboembolic events and an improvement in the quality of life. This effective concept of complement inhibition provides the rationale for ongoing studies in other complement-mediated diseases such as atypical hemolytic uremic syndrome, antibody-mediated transplant rejection and hemolytic cold agglutinin disease (CAD). In CAD, IgM autoantibodies can activate the complement system in colder areas of the body, resulting in intravascular red blood cell hemolysis. Standard therapeutic approaches are typically not effective. Some cases are mild and self-limited, but others require hospitalization and can be life-threatening. Inhibition of the terminal complement by the anti-C5 antibody eculizumab as in PNH could be a new possible therapeutic approach and long-term efficacy has already been demonstrated in one CAD patient (Röth et. al, Blood 2009). A good understanding of the pathophysiology of CAD and the proof of concept in the patient above are the rationale for the ongoing DECADE Trial for the use of eculizumab in patients with CAD.

Disclosure: Alexander Röth: Honoraria: Vortragshonorare von Alexion Financing of Scientific Research: Finanzielle Unterstützung der DECADEStudie durch Alexion

\section{Freie Vorträge Bronchialkarzinom}

\section{V953}

Notch regulates differentiation and tumorigenicity of KRAS-driven adenocarcinomas of the lung in vivo

\section{Baumgart, A. ${ }^{1}$; Mitova, N. ${ }^{1}$; Anton, M. ${ }^{2}$; Siveke, J.;} Loddenkemper, C. ${ }^{4}$; Peschel, C. ${ }^{1}$; Duyster, J. ${ }^{1}$; Dechow, T. ${ }^{1}$ ${ }^{1}$ Klinikum r.d. Isar, TU München, III. Med. Klinik, München, Germany, ${ }^{2}$ Klinikum r.d. Isar, TU München, Experimentelle Onkologie, München, Germany, ${ }^{3}$ Klinikum r.d. Isar, TU München, II. Med. Klinik, München, Germany, ${ }^{4}$ Klinikum r.d. Isar, TU München, Pathologie, München, Germany

The therapeutic options for non-small cell lung cancer (NSCLC) are limited with a poor prognosis. Thus, new compounds including g-secretase inhibitor are being tested in clinical studies. Recently we showed that Notch1 (N1) signaling promotes survival and tumorigenicity in NSCLC cells in vitro. The function of Notch2 (N2) in lung cancer however is largely unclear. We hypothesized that Notch could play an important role in the differentiation and tumorigenesis of KRAS-induced lung cancer in vivo.

To address this question we used a KRAS-driven endogenous NSCLC mouse model (LSL-KRAS ${ }^{\mathrm{G} 12 \mathrm{D}}$ ). These mice were crossed to N1 and N2 conditional knockout (KO) mice to study the role of Notch signaling in the development of adenocarcinomas. In these mice, oncogenic $\mathrm{KRAS}^{\mathrm{G} 12 \mathrm{D}}$ is transcriptionally activated by inhalative application of adenoviral Cre-Recombinase, while Notch is specifically deleted in the same cells. 
KRAS and N1-deficient KRAS tumors developed hyperplasia, adenomas and adenocarcinomas after 6 month with no significant difference in latency, morphology and number/size of tumors in the two genotypes. Surprisingly, N2 deletion led to a markedly increased carcinogenesis. Mice developed aggressive tumors within 3 months. At 6 months, mice revealed large tumor masses in the lungs. Moreover, these mice died significantly earlier. Pathologically, the KRAS and KRAS-N1 tumors showed well-differentiated tubulo-papillary adenocarcinomas. In contrast, KRAS-N2 tumors were undifferentiated and showed a high proliferation index. Further pathological and immunohistochemical analyses are ongoing.

According to our data, the LSL-KRAS ${ }^{\mathrm{G} 12 \mathrm{D}}$ mouse model is suitable to study the role of signaling pathways in lung cancer in vivo. While N1 seems to be dispensable for KRAS-driven carcinogenesis, specific deletion of N2 induces a dramatic enhancement of tumor development. Thus, N2 seems to execute differentiation and tumor suppressor function in these cells. Our data also suggests that Notch signaling is very complex and different Notch family members might have opposite functions. This is clinically important, because g-secretase inhibitors in cancer therapies might have deleterious effects on KRAS-dependent adenocarcinomas of the lung.

Disclosure: No conflict of interest disclosed.

\section{V954}

\section{Feasibility of dynamic contrast-enhanced MRI (DCE-MRI) based angiogenesis biomarker assessment in advanced NSCLC treated with erlotinib and bevacizumab}

Scheffler, M. ${ }^{1}$; Ullrich, R. ${ }^{2}$; Wetzel, T.2; Nogova, L. 3 ; Zander, T. ${ }^{3}$; Mattonet, C. ${ }^{3}$; Stoelben, E. ; Engel-Riedel, W. ${ }^{5}$ Tittgemeyer, M. ${ }^{2}$; Wolf, $\mathrm{J}^{3}$

${ }^{1}$ Klinik I für Innere Medizin, Universitätsklinikum Köln (BMBF 01KN0706), Lung Cancer Group Cologne (LCGC) am Centrum für Integrierte Onkologie (CIO) Köln-Bonn, Köln, Germany, ${ }^{2}$ Max-Planck Institut für neurologische Forschung mit Klaus-Joachim-Zülch-Laboratorien der Max-PlanckGesellschaft und der Medizinischen Fakultät der Universität zu Köln, Köln, Germany, ${ }^{3}$ Klinik I für Innere Medizin, Universitätsklinikum Köln, Lung Cancer Group Cologne (LCGC) am Centrum für Integrierte Onkologie (CIO) Köln-Bonn, Köln, Germany, ${ }^{4}$ Lungenklinik Merheim, Thoraxchirurgie, Köln, Germany, ${ }^{5}$ Lungenklinik Merheim, Onkologie, Köln, Germany

Introduction: So far, no angiogenesis-related predictive biomarkers could be established for VEGF-targeted therapy. For this purpose, dynamic contrastenhanced MRI (DCE-MRI) is increasingly evaluated in solid tumors, its use in lung cancer, however, is hampered by breathing artefacts. Within an ongoing pilot trial of sequential multimodality imaging in patients with advanced NSCLC treated with erlotinib and bevacizumab, we set out to evaluate the feasibility and the impact of sequential DCE-MRI scans for the assessment of imaging-based biomarkers for vascular permeability and perfusion (NCT01047059).

Methods: The trial was initiated in January, 2010. Patients with NSCLC stage IIIB/IV (non-SCC histology) without prior systemic treatment for NSCLC receive combined erlotinib (150 $\mathrm{mg} / \mathrm{d})$ and bevacizumab $(15 \mathrm{mg} / \mathrm{kg} \mathrm{q} 3 \mathrm{w})$ treatment for at least six weeks, and dynamic contrast-enhanced (DCE) MRI data were acquired before, after one week, and after six weeks of therapy on a 3T MRI system (Siemens ${ }^{\text {TM }}$ MAGNETOM Tim Trio) using a time-resolved 3D MRA sequence (TWIST). DCE-MRI scans are coregistrated with T1/T2 scans and corrected for respiration movements. Permeability parameters were estimated using the syngo Tissue 4D software package (Siemens AG, Erlangen, Germany). In cases of non-progression, DCE-MRI scans are repeated every six weeks until progression.

Results: Until May $1^{\text {st }}, 2010,7$ patients were enrolled in this trial, and 15 DCE-MRI scans were performed. To date, DCE-MRI images were of high quality, even giving the possibility to assess response to therapy according to RECIST 1.1. We here demonstrate an example of an early DCE-MRI scan in a patient harboring an activating EGFR mutation, with both perfusion/permeability parameters (ktrans, kep, IAUC) as well as tumor diameters demonstrating response to therapy after one week of treatment. Noteworthy, the tumor burdens in this sequence are not biased strongly by movement artefacts. This observation is stringent througout the performed scans and might be due to improved assessment software and protocols established in preclinical settings.
Conclusions: DCE-MRI is a feasible tool in assessing changes in tumor vasculature under VEGF-targeted therapy. It might also act for response evaluation according to RECIST, due to minimalization of respirative movement artefacts. Further analysis of reproducibility regarding the vasculature parameters (ktrans, kep, IAUC etc.) in 20 patients at baseline level will be presented.

Disclosure: Matthias Scheffler: Financing of Scientific Research: Unterstützung durch Roche

Jürgen Wolf: Advisory Role: Advisory board bei Roche; Honoraria: Vortragshonorare; Financing of Scientific Research: Unterstützung durch Roche

\section{V955}

\section{Dasatinib is a potent inhibitor of cancer-associated} fibroblast (CAF) proliferation and may reverse the phenotype of CAFs to a phenotype comparable to that of normal fibroblasts

Schmid, J.O. ${ }^{1}$; Haubeiß, S. ${ }^{1}$; Muerdter, T.E. ; Friedel, G. ${ }^{2}$; van der Kuip, H. ${ }^{1}$; Aulitzky, W.E. ${ }^{3}$

${ }^{1}$ Dr. Margarete Fischer-Bosch-Institute of Clinical Pharmacology and University of Tübingen, Stuttgart, Germany, ${ }^{2}$ Klinikum Schillerhoehe, Toraxchirurgie, Stuttgart, Germany, ${ }^{3}$ Robert Bosch Hospital, Hämatologie, Onkologie und Palliativmedizin, Stuttgart, Germany

It is well established that cancer associated fibroblasts (CAFs) possess permissive and promoting properties for epithelial tumor cells and therefore play a central role in tumor progression, metastasis, and probably also in sensitivity to antitumor agents. We asked whether CAFs could be reverted back to normal homeostasis by pharmacological inhibition of kinases.

We screened two CAF strains from individual lung carcinomas for their sensitivity to a panel of 160 kinase inhibitors. 5 inhibitors were identified inhibiting more than $50 \%$ of the growth in both CAF strains. Three of them are described to inhibit PDGFR at nanomolar concentrations. This finding prompted us to further test the FDA approved PDGFR inhibitors Dasatinib, Nilotinib, Sorafenib, and Imatinib. All 37 CAF strains investigated were highly sensitive to Dasatinib at clinically relevant dosages. Imatinib was slightly less effective, whereas the inhibitory effects of Nilotinib and Sorafenib were significantly less pronounced.

In a further step, we investigated possible effects of Dasatinib on the CAF transcriptome by microarray analysis of 9 individual CAF strains. The identified Dasatinib expression signature was then compared to previously described differential signatures of normal tissue associated fibroblasts (NAFs) and CAFs derived from breast cancer patients (Sadlonova et al., 2009) and to a signature of fibroblast serum response (Chang et al.2004). There was a significant overlap between genes regulated by Dasatinib and serum repression genes. More importantly, of the 313 genes downregulated by Dasatinib 64 genes were also reduced in NAFs compared to CAFs. In addition, 26 of 179 genes identified as upregulated by Dasatinib were also found to be elevated in NAFs compared to CAFs.

In summary, our data demonstrate that Dasatinib partially reverses the phenotype of CAFs to a normal fibroblast like phenotype. Therefore, targeting CAFs with Dasatinib represents a promising therapeutic principle which should be further explored.

Disclosure: No conflict of interest disclosed. 
V956

Sabarubicin (SABA) in combination with Cisplatin (DDP): Maximum tolerated dose (MTD) evaluation (phase I step) followed by a phase II step in patients (pts) with Small Cell Lung Cancer-Extensive Disease (SCLC-ED)

Cornely, O.A. ${ }^{1,2,3}$; Wolf, J.1,3; Josten, K.M. ${ }^{4}$; Müller, T.5; Dürk, H.A. ${ }^{6}$; Neumeister, W. ${ }^{7}$; Sebastian, M. ${ }^{8}$; Schroeder, M. ${ }^{9}$; Simonelli, C. ${ }^{10}$; Capriati, A. ${ }^{10}$; Karthaus, M. ${ }^{11}$

${ }^{1}$ Uniklinik Köln, Klinik I für Innere Medizin, Köln, Germany, ${ }^{2}$ Zentrum für Klinische Studien ZKS Köln (BMBF 01KN0706), Köln, Germany, ${ }^{3}$ Uniklinik Köln, COI Köln Bonn, Köln, Germany, ${ }^{4}$ Deutsche Klinik für Diagnostik, Wiesbaden, Germany, ${ }^{5}$ Medizinische Klinik III, Hofheim, Germany, ${ }^{6}$ St Marien-Krankenhaus, Hamm, Germany, ${ }^{7}$ Klinikum Marienhof, St. Joseph GmbH, Koblenz, Germany, ${ }^{8}$ Medizinische Klinik III, Mainz, Germany, ${ }^{9}$ St. Johannes-Hospital, Duisburg, Germany, ${ }^{10}$ Menarini Group, Firenze, Italy, ${ }^{11}$ Tumorzentrum München-Süd, München, Germany

Background: SABA, a 3rd generation anthracycline with promising activity as single agent in solid tumors was evaluated to establish the MTD in combination with DDP (Phase I), followed by a Phase II evaluating SABA + DDP as first line therapy in SCLC-ED pts.

Methods: In Phase I, MTD was evaluated with ascending SABA doses of 40, 60,80 and then $10 \mathrm{mg} / \mathrm{m}^{2}$ increments (day 1) together with a fixed dose of DDP $80 \mathrm{mg} / \mathrm{m}^{2}$ (day 2) in pts with solid tumors. In Phase II, SABA $80 \mathrm{mg} / \mathrm{m}^{2}$ and DDP $80 \mathrm{mg} / \mathrm{m}^{2}$ iv q21 days for 6 cycles were primarily assessed for Complete or Partial Response (CR/PR); secondarily for Overall Survival (OS), Duration of Tumor Response (DTR), Time To Progression (TTP), and safety in chemotherapy-naive SCLC-ED pts.

Results: Phase I recruited 17 pts. Best response was PR in 3 (23.1\%) pts. Dose-Limiting Toxicity (DLT) occurred in 2 of 5 pts at SABA $90 \mathrm{mg} / \mathrm{m}^{2}$, i.e. grade (G) 4 thrombocytopenia and G 4 neutropenia associated with $\mathrm{G} 3$ pyrexia. MTD for Phase II was $80 \mathrm{mg} / \mathrm{m}^{2}$. In phase II 17 males, 8 females with SCLC-ED (mean age 63 years) were enrolled. One (4\%) pt was excluded from the efficacy population for a delay of study therapy for non-medical reasons) Nineteen $(79.2 \%)$ pts. achieved CR $(1 ; 4.2 \%)$ or PR $(18 ; 75 \%), 4(16.7 \%)$ SD and $1(4.2 \%)$ progressed. Median OS, DTR and TTP were 11.6, 3.8 and 6.5 months, respectively. In Phase II treatment-related AEs referred mainly to the gastrointestinal (69 AEs in 21 (84\%) pts) or hematological system (84 AEs in 19 (76\%) pts). Premature study discontinuation occurred in $7(28 \%)$ pts due to $8 \mathrm{AEs}$. In $13(52 \%)$ pts $20 \mathrm{G} 4$ related AEs were reported, 18 of 20 were blood/ lymphatic system. In total, 2 cardiac SAEs occurred: $1 / 42(2.4 \%) \mathrm{G} 2$ heart failure and $1 \mathrm{G} 2(2.4 \%)$ tachycardia. No clinically significant signals were detected in laboratory and physical evaluation, ECG and echocardiography Fatal AEs with a suspected relationship to SABA + DDP occurred at the lowest SABA dose $\left(40 \mathrm{mg} / \mathrm{m}^{2}\right)$ in Phase I and in Phase II $\left(80 \mathrm{mg} / \mathrm{m}^{2}\right)$ in one pt. each.

Conclusion: SABA in combination with $80 \mathrm{mg} / \mathrm{m}^{2}$ DDP reached MTD at $80 \mathrm{mg} / \mathrm{m}^{2}$. SABA + DDP were effective and safe as first line treatment in SCLC-ED, which appears comparable to standard regimens.

Disclosure: No conflict of interest disclosed.
V957

Fluoro-L-Thymidine (FLT) uptake in baseline positron emission tomography (PET) as a prognostic marker in patients with advanced non-small cell lung cancer (NSCLC) treated first-line with erlotinib

Scheffler, M. ${ }^{1}$; Zander, T. ${ }^{1}$; Nogova, L. ${ }^{1}$; Kobe, C. ${ }^{2}$; Dietlein, M. ${ }^{2}$; Papachristou, I. ${ }^{1}$; Querings, S. ${ }^{3}$; Thomas, R. ${ }^{4}$; Stoelben, E. Engel-Riedel, W. ${ }^{6}$; Neumaier, B. ${ }^{7}$; Wolf, J. ${ }^{1}$

${ }^{1}$ Klinik I für Innere Medizin, Universitätsklinikum Köln, Lung Cancer Group Cologne (LCGC) am Centrum für Integrierte Onkologie (CIO) Köln-Bonn, Köln, Germany, ${ }^{2}$ Klinik und Poliklinik für Nuklearmedizin,

Universitätsklinikum Köln, Centrum für Integrierte Onkologie (CIO) KölnBonn, Köln, Germany, ${ }^{3}$ Max-Planck Institut für neurologische Forschung mit Klaus-Joachim-Zülch-Laboratorien der Max-Planck-Gesellschaft und der Medizinischen Fakultät der Universität zu Köln, Köln, Germany, ${ }^{4}$ Laboratory of Translational Cancer Genomics (LTCG), Centrum für Integrierte Onkologie $(\mathrm{ClO})$ Köln-Bonn und Max-Planck-Institut für neurologische Forschung, Köln, Germany, ${ }^{5}$ Lungenklinik Merheim, Thoraxchirurgie, Köln, Germany, ${ }^{6}$ Lungenklinik Merheim, Onkologie, Köln, Germany, ${ }^{7}$ Max-Planck Institut für neurologische Forschung mit KlausJoachim-Zülch-Laboratorien der Max-Planck-Gesellschaft und der Medizinischen Fakultät der Universität zu Köln, Radiochemie, Köln, Germany

Introduction: Since the introduction of targeted therapy into the treatment of advanced NSCLC molecular imaging tools gain in importance for assessment of pharmacodynamics, prognosis and prediction of therapeutic outcome. We evaluated the prognostic value of $3^{\prime}-$ deoxy-3'-[18F]fluoro-L-thymidine (FLT) uptake measured by positron emission tomography (PET) as a noninvasive proliferation marker in patients with metastatic non-small cell lung cancer (NSCLC) prior to systemic therapy with erlotinib.

Methods: We retrospectively analysed FLT uptake in 41 chemonaive patients with advanced NSCLC (stage IIIBwet/IV) treated with erlotinib first-line prior to systemic therapy. The peak SUV of the lesion with the highest SUV(maxSUVpeak) as well as sex, age, ECOG performance state at presentation, EGFR mutational status and adenocarcinoma/BAC histology were analyzed in univariate analysis using Kaplan-Meier statistics and log rank test for its association with survival. Multivariate analysis was performed using Cox regression.

Results: Twenty-nine (69\%) of the forty-one patients died until date of reporting. Median follow-up was 16.4 months. In univariate analysis, patients with a maxSUVpeak $<3.1$ had a significantly better overall survival $(15.9$ months [95\% CI 9.3 to 22.5 months]) compared to patients with a peak SUV $\geq 3.1$ ( 3.3 months [95\% CI 0 to 6.9 months]) ( $\mathrm{p}=0.004$, Log-Rank). In addition, ECOG performance status was significantly associated with overall survival $(\mathrm{p}=0.004$ $\log$ rank test). In this cohort none of the clinical parameters gender, histology, age or EGFR mutational status were significantly associated with overall survival. In multivariate analysis including ECOG and maxSUVpeak, maxSUVpeak was a significantly strong prognostic factor for overall survival $(\mathrm{p}=0.019)$.

Conclusions: FLT uptake of the most active tumor manifestation site is a strong prognostic marker for overall survival among patients with advanced NSCLC treated with erlotinib first-line.

Disclosure: Matthias Scheffler: No conflict of interest disclosed. Jürgen Wolf: Advisory Role: Beratungstätigkeit für Roche; Financing of Scientific Research: Finanzierung wissenschaftlicher Untersuchungen durch Roche. 
V958

A phase II double-blind study to investigate efficacy and safety of the triple angiokinase inhibitor BIBF 1120 in patients with relapsed advanced non-small cell lung cancer (NSCLC)

Reck, M. ${ }^{1}$; Kaiser, R. ${ }^{2}$; Eschbach, C. ${ }^{3}$; Stefanic, M.4; Gaschler-Markefski, B. ${ }^{4}$; Gatzemeier, U. ${ }^{1}$; von Pawel, J. ${ }^{5}$

${ }^{1}$ Hospital Grosshansdorf, Hamburg, Germany, ${ }^{2}$ Boehringer Ingelheim Pharma GmbH \& Co. KG, Clinical Research, Biberach, Germany,

${ }^{3}$ Asklepios Hospitals Harburg, Hamburg, Germany, ${ }^{4}$ Boehringer Ingelheim Pharma GmbH \& Co. KG, Biberach, Germany, ${ }^{5}$ Asklepios Hospitals, München-Gauting, Germany

Background: Most patients with NSCLC present with advanced disease and poor prognosis. VEGFR is a major regulator of angiogenesis and VEGFR dysregulation has been implicated in NSCLC development. PDGFR has a role in promoting angiogenesis, tumour growth and metastasis. FGFR is also an important regulator of tumour blood vessels. BIBF 1120 is a potent, oral, triple angiokinase inhibitor of VEGFR 1-3, FGFR 1-3 and PDGFR $\alpha / \beta$. This study assessed the efficacy, safety and tolerability of two BIBF 1120 doses in patients with stage IIIB/IV NSCLC.

Methods: Patients with an ECOG score of 0-2 with locally advanced or metastatic relapsed NSCLC who had failed at least one line of chemotherapy were randomized to daily BIBF $1120250 \mathrm{mg}$ BID or $150 \mathrm{mg}$ BID. Primary endpoints were PFS and objective tumour response (RECIST 1.0). QOL was measured with the EORTC QLQ-C30 and its lung cancer module EORTC QLQ-LC13 (R99-1213). The EORTC QLQ-C30 and EORTC OLQ-LC13 were completed by patients at every treatment course. QOL was assessed by the time to deterioration (defined as a 10-point decrease from the baseline score) for global health status (Questions 29 and 30 in QLQ-C30), cough (Question 1 in QLQL-C13), dyspnoea (Question 8 in QLQ-C30), and pain (Questions 9 and 19 in QLQ-C30). Time to deterioration was analyzed similarly to PFS.

Results: 37 of 73 patients were randomized to receive $150 \mathrm{mg}$ BID and 36 to receive $250 \mathrm{mg}$ BID. Median overall PFS was 6.9 weeks, with no significant difference between treatment arms. Median OS was 21.9 weeks. Patients with ECOG 0-1 ( $\mathrm{n}=56)$ had a median PFS of 11.6 weeks and a longer median OS of 37.7 weeks compared to patients with ECOG 2. Tumour stabilization was achieved in $46 \%$ of patients (ECOG 0-1, 59\%), with one confirmed PR (250 mg BID group). Patients with ECOG 0-1 had a median time to deterioration for pain of 85 wks. The median times differed between the two treatment groups (126 wks and 45 wks for the $150 \mathrm{mg}$ BID and $250 \mathrm{mg}$ BID cohorts, respectively). The most commonly reported drug-related AEs over both dose groups were nausea $(57.5 \%)$, diarrhoea $(47.9 \%)$, vomiting $(42.5 \%)$, anorexia (28.8\%), abdominal pain (13.7\%) and reversible elevations of ALT (13.7\%) and AST $(9.6 \%)$

Conclusions: Continuous treatment with BIBF 1120 was well-tolerated with no difference in efficacy between treatment arms. The observed PFS and OS with single agent treatment in advanced NSCLC warrants further exploration in controlled clinical trials.

Disclosure: Martin Reck: Honoraria: Advisory role for Hoffmann-La Roche (compensated), Lilly (compensated), Merck (compensated), AstraZeneca (compensated), Novartis (compensated)

Joachim von Pawel: Honoraria: Consultant or advisory role for Boehringer Ingelheim.

\section{Freie Vorträge}

\section{Immuntherapie 2}

\section{V959}

Antibody targeting of epithelial carcinoma associated fibroblast activation protein blocks cellular proliferation, migration and invasion

Bauer, S. ${ }^{1}$; Fischer, E. ${ }^{2}$; Wüest, T. ${ }^{3}$; Scott, A.M. ${ }^{4}$; Schibli, R. ${ }^{2}$; Ritter, G. ${ }^{5}$; Old, L.J. ; Renner, C.

${ }^{1}$ Nationales Centrum für Tumorerkrankungen, Medizinische Onkologie, Heidelberg, Germany, ${ }^{2}$ Paul Scherrer Institut, Villingen, Switzerland, ${ }^{3}$ Universitätsspital Zürich, Zürich, Switzerland, ${ }^{4}$ Ludwig Institute for Cancer Research, Melbourne, Australia, ${ }^{5}$ Ludwig Institute for Cancer Research, New York, United States

Introduction: Activated fibroblasts in the stroma of epithelial carcinomas are key mediators of cancer initiation, progression, and metastasis. Their phenotype is characterized by secretion of various growth factors and cytokines, and, amongst others, high expression of the Fibroblast Activation Protein (FAP) on the cell membrane. FAP is a type II serine protease that also has collagenase activity. FAP contributes to the tumor architecture by remodeling of extracellular matrix (ECM) through its proteolytic activity in concert with CD26, b1-integrin, and MMP-1. Thereby, functional impairment of FAP-related bioactivities is recognized to influence malignant growth and invasive cell behavior. Extent of FAP-expression is significantly correlated with a higher risk of tumor recurrence and death in pancreatic carcinoma and has been used as a prognostic marker for colorectal cancer. Since FAP is typically absent from normal adult tissue, it has long been suggested to be a promising therapeutic target.

Methods: We selected novel anti-FAP antibodies from a nonimmunized phage library expressing antibody Fab fragments. Binding of most frequently identified clone, ESC11, to FAP was measured by surface Plasmon resonance. Clones were converted into human $\operatorname{IgG}$ antibodies and chosen for upscaled production. Reactivity of anti-FAP IgG to activated fibroblasts was assessed by flow cytometry. Interference of novel antibodies with FAP-mediated proinvasive effects was demonstrated by proliferation assays and invasion of ECM.

Results: The novel human antibody binds to FAP at low nanomolar affinities and specifically target activated fibroblasts. Crosslinking of FAP by ESC11 antibody significantly reduced its cell surface expression by rapid internalization. Such antibody-mediated conversion of a FAP-positive into a FAPnegative phenotype resulted in significant reduction of proliferation, migration, and invasion of activated fibroblasts.

Conclusions: Since malignant and invasive behaviour of epithelial cancers depends on activated fibroblasts, our data strongly provide evidence for clinical development of novel human anti-FAP antibody.

Disclosure: No conflict of interest disclosed. 
V960

\section{Results of first-in-man phage idiotype vaccine study in patients with multiple myeloma}

\section{Röhnisch, T.'; Nagel, W. 2; Boehm, T.3.; Donceau, M. ${ }^{3}$; Bourquin} C. ${ }^{4}$; Röhnisch, D.A. ${ }^{3}$; Rutz, B. ${ }^{3}$; Emmerich, B. ${ }^{1}$; Oduncu, F.S. ${ }^{3}$

${ }^{1}$ Onkologie Nussbaumstrasse, Gemeinschaftspraxis Prof. Dr. B. Emmerich, Prof. Dr. P. Maubach, Dr. T. Röhnisch, München, Germany, ${ }^{2}$ Deutsches Forschungszentrum für Gesundheit und Umwelt (GSF), Helmholtz Zentrum München, Neuherberg, Germany, ${ }^{3}$ Klinikum der Universität München, Medizinische Klinik, Campus Innenstad, Abt. für Hämatologie-Onkologie, München, Germany, ${ }^{4}$ Klinikum der Universität München, Medizinische Klinik - Campus Innenstad, Abt. für Klinische Pharmakologie, München, Germany

Idiotypes (Id) expressed by tumor cells in B-cell malignancies can be regarded as tumor-specific antigens and may therefore be used as a target for vaccine immunotherapy. In this phase I/II study we have evaluated the feasibility, safety and response of phage idiotype vaccination in patients with relapsed multiple myeloma (MM) using phage displayed Id-specific proteins as immunogens in combination with GM-CSF as immunoadjuvants.

Study Design: Patients received a total of 6 intradermal/subcutanous (i.d./s.c) immunizations of phage idiotype vaccines at day $1,7,14$ and at week 4,8 an 12. The vaccine dose started from $0.25 \mathrm{mg}$ for the first 5 patients and was escalated to $1.25 \mathrm{mg}$ (patient 6-10) and $2.5 \mathrm{mg}$ (patient 11-15) if no doselimiting toxicity was observed. Each vaccine contained $0.2 \mathrm{mg}$ GM-CSF (Leukomax®) as adjuvant and soluble $0.2 \mathrm{mg} \mathrm{KLH}($ Immucothel®) as control.

Patient Characteristics: 15 patients who had previously been treated with high-dose chemotherapy (HDT) followed by peripheral blood progenitor cell (PBPC) transplantation entered this study. All patients were in a beginning relapse after high dose therapy at the time of vaccination. Patients with fulminant relapse or patients with active infection and hematopoetic insufficiency after HDT were excluded.

Results: The results of this first-in-man phase I/II trial for phage-based idiotype vaccination clearly demonstrated the feasibility, safety and excellent tolerability of the phage vaccination whereas local skin reactions were the most frequent side effects. Furthermore, we were able to gather precious data regarding the immunology of phage vaccines in man. We could confirm our preclinical data, that phages are good immunogens, capable of inducing a strong immune response mainly of IgG1-type and therefore enabling ADCC and CDC immune response. Altogether these data suggest, that phage idiotype vaccines has the potential to exert a beneficial effect especially in patients with minimal residual disease. Strikingly, the immune response of phages compared to the well-known KLH particles showed > 10 time higher antibody titer than KLH, which emphasize the prominent immunogenicity of the phages. Overall these data pinpoint to a promising role of phage vaccination for the immunotherapy of cancer but further studies are necessary to evaluate the full potential of phage vaccines.

Disclosure: No conflict of interest disclosed.

\section{V961}

Overcoming negative immune regulatory feedback loops in dendritic cells: basis for a next generation of cancer vaccines

Dohnal, A. ${ }^{1}$; Luger, R. ${ }^{1}$; Halfmann, A. ${ }^{1}$; Vittori, S. ${ }^{1}$; Vakookaran, S. ${ }^{1}$; Schuh, C. ${ }^{1}$; Erhart, F. ${ }^{1}$; Gaestel, M. ${ }^{2}$; Felzmann, T. ${ }^{1,3}$

${ }^{1}$ St. Anna Children's Cancer Research Institute, Laboratory for Tumor Immunology, Vienna, Austria, ${ }^{2}$ Hannover Medical School, Institute of Biochemistry, Hannover, Germany, ${ }^{3}$ Trimed Biotech $\mathrm{GmbH}$, Vienna, Austria

Introduction: Anti-tumour immune therapy is based on the notion that cancer may be viewed as the symptom of an immune deficiency frequently occurring at the level of dendritic cells (DC), which orchestrate all forms of immune responses. This realisation resulted in the development of DC based cancer vaccines, the first of which have recently gained regulatory approval. However, so far DCs do not seem to have fulfilled their potential. Critically, DCs not only trigger immunity but also down-modulate immune responses to prevent them from running out of control. So far, little attention has been paid to these negative immune regulatory feedback loops and their interference with the development of powerful anti-tumour immune responses.

Methods: We used expression profiling and RNA interference to spot DC molecules involved in negative regulation of immune responses. Using allogeneic mixed leukocyte reactions we measure T-cell proliferation in co-cultures with DCs engineered for down-modulation of target molecules. In addition, selected target molecules were studied in DCs from knockout mice using TCR transgenic OT-I and/or OT-II T-cells as effectors.

Results: The knockdown of seven molecules (FOXP1, PHF11, JAK1, MK2, RGS16, similar to IRP1, IFI44L) in human DCs resulted in up to ten-fold improved proliferation of allogeneic CD4 and CD8 T-cells in comparison to unmodified DCs. This surpassed the stimulatory capacity of DCs in which the known immune suppressive molecules STAT3, IRF4, or IDO were blocked. Using cytokine bead arrays we found a strong type 1 polarisation profile. Particularly conclusive results were obtained for MAP kinase (MK) 2 blockade in human and mouse experiments. DCs from murine MK2 knockout mice significantly exceeded the stimulatory capacity of wild type DCs for OT-I and/ or OT-II T-cells.

Conclusions: The activation of DCs is well understood. In particular TLR activated, IL-12 secreting, and strongly type 1 polarising DCs that we currently explore in a randomised clinical efficacy trial for the treatment of brain cancer utilise the full immune stimulatory power of DCs. Further improving the potency of DC cancer vaccines may, however, be accomplished targeting key molecules of negative immune regulatory feedback mechanisms. We could demonstrate that among other molecules, MK2 is a promising candidate for targeting in genetically engineered DCs that may be the active ingredient in the next generation of cancer vaccines.

Disclosure: Alexander Dohnal: No conflict of interest disclosed.

Thomas Felzmann: Employment or Leadership Position: Trimed Biotech $\mathrm{GmbH}$; Financing of Scientific Research: Finanzierung der klinischen Forschung durch Trimed Biotech GmbH

\section{V962}

\section{Serological profiling of patients with different} hematological malignancies before and after RHAMM-R3 peptide vaccination and correlation to specific $T$ cell responses and clinical findings

Greiner, J. ${ }^{1}$; Hofmann, S. ${ }^{1}$; Giannopoulos, K. ${ }^{1}$; Rojewski, M. T. ${ }^{2}$; Babiak, A. ${ }^{1}$; Ushmorova, D. ${ }^{1}$; Bunjes, D. ${ }^{1}$; Doehner, H. ${ }^{1}$; Schmitt, A. ${ }^{3}$ Schmitt, M. $^{3}$

${ }^{1}$ University of UIm, Department of Internal Medicine III, UIm, Germany, ${ }^{2}$ University of Ulm, Institute of Clinical Transfusion Medicine, UIm, Germany, ${ }^{3}$ University of Rostock, Department of Internal Medicine III, Rostock, Germany

We performed a RHAMM-R3 peptide vaccination trial using 300 $\mu$ g and $1000 \mu \mathrm{g}$ peptide for 25 patients with AML, MDS, multiple myeloma (MM) and CLL overexpressing RHAMM. In the first cohort (10 AML, MDS and MM

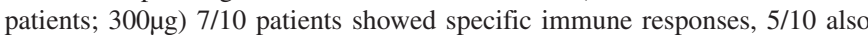
positive clinical effects. In the second cohort (6 CLL patients; 300 $\mu$ g) $80 \%$ showed immune responses and in the third cohort (10 AML, MDS and MM patients; $1000 \mu \mathrm{g}$ ) immune responses were detected in 4/9 and clinical effects in $3 / 9$ patients.

In this work, we performed a serological profiling from cohort 1 and 3 using a proteome array profiling before and after peptide vaccination to detect cytokine and chemokine regulation. 36 cytokines, chemokines and acute phase proteins were measured. Moreover, we investigated serological immune responses detected by a RHAMM-specific ELISA and we correlated these results to specific $\mathrm{T}$ cell responses measured by ELISpot assays for interferon gamma and Granzyme B and tetramer staining. These results were also compared to the frequency of regulatory T cells. Nevertheless, ELISA was established to measure IL-2 and IL-10. 11/19 patients showed specific T cell responses, 4 of these 11 had a pre-existing positive serological immune response against RHAMM detected by ELISA. All of these four patients developed strong specific CD8+ T cell responses detected by ELISpot assays and tetramer staining. Three of these patients showed positive clinical effects. For effective elimination of malignant cells by specific $\mathrm{T}$ cells a co-activation of CD4+ and CD8+ T cells might be relevant. As expected, nonamer peptide 
vaccination did not result in the induction of humoral immune responses. In ELISA we measured IL-2 and IL-10 levels in the sera. While IL-10 levels remained at a low level, we detected an increase of IL-2 up to five-fold of the initial levels in 4/10 patients. All of these patients with an increase of IL-2 showed a positive clinical reaction. Different cytokines analyzed by proteome profiling showed a significant up- or down-regulation due to vaccination in contrast to the pretreatment level. IL-1ra and CD40 ligand were increased in patients with specific $\mathrm{T}$ cell responses.

Taken together, RHAMM-R3 peptide vaccination induced both immunological and positive clinical effects. Co-existence of immune responses of CD4+ $\mathrm{T}$ cells against the target RHAMM and also cytokine regulation seems to be important for an induction of strong immune responses of CD8+ T cells.

Disclosure: No conflict of interest disclosed.

\section{V963}

\section{Host interferon- $\gamma$ signaling and cross-presentation is required for elimination of antigen-loss variants of high- grade B cell lymphomas: Implications for adoptive T-cell therapy}

Gerbitz, A. ${ }^{1}$; Sukumar, M. ${ }^{2}$; Helm, F. ${ }^{3}$; Wilke, A. ${ }^{3}$; Friese, C. Fahrenwaldt, C. ${ }^{3}$; Lehmann, F. ${ }^{5}$; Loddenkemper, C. ${ }^{6}$; Kammertöns, T. ${ }^{3}$; Mautner, J.7; Blankenstein, T. ${ }^{3}$; Bornkamm, G. ${ }^{5}$ ${ }^{1}$ Universität Erlangen, Hämatologie/Onkologie, Erlangen, Germany, ${ }^{2} \mathrm{NIH}$, Sugey Branch, Bethesda, United States, ${ }^{3}$ Charite CBF, Institut für Immunologie, Berlin, Germany, ${ }^{4}$ Charite $\mathrm{CBF}$, Institute für Immunologie, Berlin, Germany, ${ }^{5}$ Helmholtz Zentrum München, Institut für klinische Molekularbiologie und Tumorgenetik, München, Germany, ${ }^{6}$ Technische Universität München, Institut für Pathologie, München, Germany, ${ }^{7}$ Technische Universität München, Kinderklinik, Klinische Kooperationsgruppe, München, Germany

Introduction: Tumor cells are characterized by genetic changes that frequently give rise to the synthesis of mutated proteins that may serve as targets for rejection by T-cells. The ability of the immune system to fight hematologic malignancies has been demonstrated in two paradigmatic settings: allogeneic stem cell transplantation (aSCT) for treatment of CML and adoptive T-cell therapy for the treatment of EBV induced posttransplant lymphoproliferative disease. Both have in common that foreign antigens are the targets of T-cell response. Contrary to CML, the benefit of aSCT for treatment of high-grade lymphomas is not well established. Several studies did not reveal a GvL effect for diffuse large B-cell lymphomas and Burkitt's lymphoma. We therefore established a lymphoma model that would allow us to investigate mechanisms of T-cell mediated rejection in hematopoietic tumors.

Methods: Using a murine model of high-grade B-cell lymphoma we studied T-cell mediated rejection upon introduction of a foreign antigen into lymphoma cells. Chicken Ovalbumin (OVA) was cloned into a MSCV based retroviral vector and transduced lymphoma cells were transferred into genetically different mouse strains.

Results: Expression of OVA in lymphoma cells resulted rejection after transfer in $80 \%$ of recipient mice. Rejection was dependent on the presence of IFNg since IFNg deficient mice failed to reject lymphoma cells and $90 \%$ of mice died due to progression. Outgrowing lymphomas surprisingly displayed antigen loss indicating that killing of antigen positive cells was sufficient but "bystander eradication" of loss variants was abolished. Identical results were observed when recipient mice were devoid of the IFNg-receptor indicating the importance of the stroma in bystander eradication. Recipient mice unable to crosspresent OVA derived peptides displayed reduced T-cell responses and survival was significantly inferior compared to wildtype mice.

Conclusion: This study demonstrates for the first time that T-cell mediated rejection of haematologic malignancies such as high grade B-cell lymphoma is dependent on stromal antigen presentation. The elimination of antigen expressing lymphoma cells is dependent on IFNg and IFNg needs to act on the stroma for sufficient elimination of antigen loss variants. Thus, treatment strategies using chimeric T-cells receptors for adoptive T-cell therapy or antibodies alone may not be sufficient since antigens crosspresented by stromal cells are not recognized.

Disclosure: No conflict of interest disclosed.

\section{V964}

\section{CCL19 as an adjuvant for Her2/neu DNA vaccination: Gene gun immunization is superior to intramuscular} vaccination and conveys long-term tumour protection

\author{
Nguyen Hoai, T.1,2; Hohn, O. ${ }^{3}$; Höpken, U.E.' ${ }^{2}$ Lipp, M. ${ }^{2}$;
} Norley, S. ${ }^{3}$; Dörken, B. ${ }^{1,2}$; Pezzutto, A. ${ }^{1,2}$; Westermann, J.1,2 ${ }^{1}$ Department of Hematology, Oncology and Tumour Immunology, CharitéUniversity Medincine Berlin, Campus Berlin-Buch and Campus VirchowKlinikum, Berlin, Germany, ${ }^{2}$ Max-Delbrück-Center for Molecular Medicine, Berlin, Germany, ${ }^{3}$ Robert Koch Institute, Berlin, Germany

Introduction: We have previously shown that coexpression of chemokines such as CCL19 and CCL21 is a strategy to enhance antitumour immunity by intramuscular (i.m.) DNA vaccination. The aim of this study was to compare the efficacy of i.m. DNA delivery with intradermal (i.d.) immunization by gene gun and to confirm CCL19 as a potent adjuvant in a Her2/neu mouse tumour model.

Methods: Mice were immunized with plasmid DNA twice i.m. (100mg DNA) or i.d. by gene gun (1mg DNA) on days 1 and 15 . Administration of plasmid DNA (pDNA) encoding Her2/neu (pHer2) was compared with pHer2 plus pCCL19, pHer2 plus pCCL19 plus pGM-CSF, pCCL19 or pGM-CSF alone and mock vector. Tumour challenge was performed subcutaneously on day 25 with syngeneic Her2/neu+ tumour cells (D2F2E2). The percentages of tumour-free mice as well as tumour growth were monitored thereafter. Humoral and cellular anti Her2/neu immune responses were assessed by FACS-ELISA, IFN-g/IL-4 Elispot assays and pentamer staining.

Results: Intradermal injection by gene gun led to a substantial enhancement of tumour protection by the DNA vaccine as compared with i.m. immunization: 125 days after tumour challenge $40 \%$ of mice immunized i.d. with pHer 2 alone remained tumour-free as compared with $0 \%$ after i.m vaccination. The protective effect of the vaccine was enhanced by coadministration of pCCL19 (70\% tumour-free mice as compared with $30 \%$ after i.m. vaccination) and pCCL19 plus pGM-CSF (100\% tumour-free mice as compared with $25 \%$ after i.m. immunization). Immunization with pCCL19 or mock vector alone did not result in tumour protection. Gene gun immunization against Her2/neu induced both humoral and $\mathrm{T}$ cellular immune responses. T cell responses were mainly directed against epitopes from the extracellular domain of Her $2 /$ neu. Responses of CD8+ T cells against the Her2p63 epitope, as analysed by pentamer staining, showed that gene gun immunization is much more efficient than i.m. vaccination ( $5.4 \%$ vs. $0.03 \%$ pentamer-positive PBMC).

Conclusion: CCL19 is a potent adjuvant for DNA vaccination and is able to amplify protective antitumour immunity induced by a Her2/neu DNA vaccine. Compared to i.m. immunization, gene gun delivery leads to a substantial improvement of long-term tumour protection. Standardization of vaccination is more readily achieved with the gene gun which would be important in a clinical setting. DNA vaccines against Her2/neu may play a future role in the treatment of Her2/neu+ MRD breast cancer.

Disclosure: No conflict of interest disclosed.

\section{Kompetenznetz Leukämien Neue Trends in der Leukämie}

\section{V968}

\section{Registries for leukaemia patients: Concepts and purposes}

Mansmann, $U^{1}$

${ }^{1}$ IBE, München, Germany

Patient registries are systematic collections of clinical experience and useful to derive key quantities for health systems research and the clinical work-up of a patient. They provide relevant input to health systems research, patient data based meta-analyses, and many other sorts of studies in clinical epidemiology. Furthermore, they provide information for important quantities used in HTA reports.

Their data stems from two qualitatively different sources: Patients treated in clinical routine or in clinical studies. While the increasing use of clinical information systems enables an efficient data collection based on the clinical routine, this information is less precise and of restricted granularity. The information collected from study patients is more expensive but also more refined and of higher value. Therefore, both sources can not be used to answer 
the same questions. Further aspects which steer new concepts for registries are the ubiquity of complex molecular information and the combination of patien registries with biobanks.

We discuss strategies for patient registries developped to meet new challenges in biomedical research. We present an IT infrastructure for collaborative clinical research which enables cooperating centres or study groups to organise joint research projects in modern clinical epidemiology. We exemplify the problems of marker validation and individualized therapeutic approaches using the developing registry infrastructure for study patients within German leukaemia study groups. We discuss the combination of information from registries for routine patients and registries of study patients. This also touches the problem of pseudonymisation and trust centres.

The talk concludes that registries can not replace study based clinical research But, registries of clinical studies allow a new scope of clinical research which goes far behind the possibilities of routine patient registries. The infrastructure of these cooperative study registries enables clinical epidemiologists to derive new instruments for diagnosis and prognosis as building stones for individualized medicine as well as contribute to the elucidation of the cellular basis of disease processes. Contrasting their well documented study based therapeutic results with the outcome in clinical routine patients defines the needed improvement in the standard work-up of patients.

Disclosure: No conflict of interest disclosed.

\section{Arbeitskreis Sitzung \\ Rehabilitation von Patienten mit gastrointestinalen Tumoren \\ V976 \\ Rehabilitation of patients with gastrointestinal tumours after chemotherapy}

\section{Crevenna, R. ${ }^{1}$; Wiltschke, C. ${ }^{2}$; Gaiger, A. ${ }^{2}$; Marosi, C. ${ }^{2}$; Keilani, M. ${ }^{1}$; Mähr, B. ${ }^{1,3}$ \\ ${ }^{1}$ Universitätsklinik für Physikalische Medizin und Rehabilitation der Medizinischen Universität Wien, Wien, Austria, ${ }^{2}$ Universitätsklinik für Innere Medizin I der Medizinischen Universität Wien, Wien, Austria, \\ ${ }^{3}$ Therapiezentrum Rosalienhof, Bad Tatzmannsdorf, Austria}

Introduction: Patients with gastrointestinal tumours after chemotherapeutic treatment often show a decreased quality of life with impaired physical performance, mental health, emotional role functioning, and social participation. Most patients show daily fatigue and deficits in muscular strength and endurance capacity. Impaired nutrition also can lead to loss of muscle mass (sarcopenia) and cachexia. Furthermore, they can show polyneuropathy and sensorimotor deficits with the risk of falls. Bodily pain can be an important problem for these patients, and many of them are suffering from fear and impaired mental health. Some patients can show faecal disorders such as incontinence or constipation.

Methods: This presentation gives an overview about the rehabilitation goals and possibilities in this patient group. An interdisciplinary rehabilitation concept is described.

Results: A rehabilitation concept for this patient group should include an exercise program to increase physical performance and quality of life. Active strength exercise is intended to increase muscular strength and muscle mass, and active aerobic exercise aims to increase endurance capacity. For patients who are unable or not allowed to perform active exercise, neuromuscular electrical stimulation (NMES) can be a modality to increase muscle strength Physiotherapy can improve mobility and sensorimotor deficits. Nutrition helps to gain weight and to increase muscle mass. Biofeedback-training is effective in stress-management and in the treatment of incontinence or other faecal disorders. Further physical therapies such as electrotherapy, mechanotherapy (massage) and thermotherapy should be prescribed according to the individual's state. Psycho-oncological interventions and treatment can help in a time of sadness and fear, and to regain self-confidence and self-competence. Due to the fact that cancer patients often reveal symptoms which are associated to their disease, a permanent back-up of an oncologist seems to be necessary during the rehabilitation process.

Conclusions: Rehabilitation concepts for this patient group should be interdisciplinary. Different specialities such as Internal Medicine and Oncology, Physical Medicine and Rehabilitation, Psycho-Oncology, and others should work in an interdisciplinary team-process. Other associated professions (phys- iotherapists, occupational therapists, nutritionists, psychologists, social worker) complete the so called multi-professional rehabilitation team. During rehabilitation, patients should gain self-confidence and independence and to be no longer passive objects of diagnostic and therapeutic measures, but active participants in the rehabilitation process. Therefore, the rehabilitation goals should be to improve physical performance, mental health and social participation, all leading to increased quality of life.

Disclosure: No conflict of interest disclosed.

\section{Arbeitskreis Sitzung Die Angst des Krebspatienten}

\section{V979}

Fear in cancer patients - realistic fear or anxiety disorder?

Heußner, $P .^{1}$

${ }^{1}$ Klinikum der Universität München - Großhadern, Med. Klinik III - PsychoOnkologie, München, Germany

With the diagnosis of cancer disease life changes for most people dramatically. The confrontation with existential health threat might be the first time in one's life. The first association to the diagnosis of cancer is suffering and dying. On the other hand progress in cancer treatment leads to higher numbers of cancer survivors with special psychologic impact as fear of disease recurrence for several years. Recent studies found up to $56 \%$ of moderate to high levels of future-oriented fears in cancer patients.

Therefore in oncology we have to deal with different fears of our patients. We have to differentiate the thoughts of our patients. These can be

- adequate reaction to realistic health threat

- psychiatric syndrome as an anxiety disorder subdivided in

- adjustment disorder

- generalized anxiety disorder

- panic disorder or

- phobic anxiety or an adequate or inappropriate

- fear of cancer progression.

To avoid unnecessary distress of the patients the oncologist is the most important person to recognize the various manifestation of fears. He can evaluate the different thoughts of his patients and allay inadequate fears. Sometimes further or corrected information concerning the expected course of disease is needed. In other cases pharmacotherapy, ressource oriented psychotherapy, creative therapies or relaxation techniques are indicated as the appropriate therapy. In the rehabilitation setting group therapies and patient education is well established as prevention. These interventions are focussing on coping strategies. All efforts has the goal to reduce psychologic distress, to prevent secondary psychiatric disorder and to stabilize quality of life.

Disclosure: No conflict of interest disclosed.

\section{V980}

\section{Fear in cancer patients - the oncologist's perspective}

\section{Koenigsmann, M. ${ }^{1}$}

${ }^{1}$ Schwerpunktpraxis und Tagesklinik für Hämatologie und Onkologie, Hannover, Germany

For many patients, the diagnosis of cancer means an existential threat. The outlook to death and suffering, to a loss of relationships and normal functions of life causes fear as an adequate reaction. The oncologist's primary task is to restore or at least to improve the health condition of his patient and thereby to take away the primary cause of his or her fear. Dealing with the fear of death - independent of a given disease - is finally a spiritual matter.

Fear, in conjunction with a proper understanding of the disease, can serve as a motivation for the patient to be compliant towards diagnostic and therapeutic measures. However, since standing in fear is a humbling situation, the physicist has a great responsibility to carefully deal with this state of mind.

Specific situations in the course of a cancer patient elicit fear. Some of these include the notification of the new diagnosis, the anticipation of therapyinduced side effects, a relapse of cancer, a switch from curative to palliative treatment and even the upcoming of a regular follow-up examination. 
Fear in cancer patients is highly prevalent, but oftentimes not admitted and therefore unnoticed. It may become apparent through aggressiveness, speechlessness, or a certain facial expression. Dealing with the patients fear requires perceptiveness, experience, knowledge, courage and patience on the side of the oncologist.

In some patients, fear seems to the oncologist as being inadequate and is perceived as anxiety. In such cases we need to distinguish whether we lack understanding for our patient or whether there is a need for additional treatment including psychotherapy, relaxation techniques, or pharmacotherapy.

During the session, we will consider some of these situations and show specific approaches.

Disclosure: Michael Koenigsmann: Financing of Scientific Research: Roche, Amgen, Medac

\section{V981}

\section{Space for creation inspite of anxiety and loss of autonomy- an art therapy intervention (KSKT®) in psycho-oncology}

\section{Hopf, A.M. ${ }^{1}$}

${ }^{1}$ Klinikum der LMU, Campus München-Großhadern, Medizinische Klinik III, Psycho-Onkologie, München, Germany

Introduction: Art therapy is the therapeutic use of making art. This tool can support cancer patients during and after their medical treatment. Art techniques like painting can offer the possibility of self-representation in a picture. Pictures are the basis of self-understanding in main areas of the patients life. Besides cancer patients may loose the capability of self-regulation in certain areas:

1. Self-and bodyimage, which undermines the patients self-capability and physical integrity

2. Inner and outer family relations, which may be destabilized

3. Functions and roles in society and world of work, which cause self-image changes and material instability

Subject: The KSKT® (germ.: kurze strukturierte Kunsttherapie/engl.: short structured art therapy) supports the use of art therapy for distressed cancer patients and the non-verbal process of identifying, structuring and organizing internal and external representations such as self-image, needs of protection and support, related to social and other resources. Anxiety and loss of autonomy can be compensated. The aim of this study was to gain knowledge on how women react to the KSKT® intervention and subjectively evaluate the intervention. Another aim is to learn which kind of needs women have during their cancer treatment and whether the KSKT® intervention responds to these. The study population consisted of 20 adult women in an acute hospital who had heterogeneous cancer diagnoses. Patients were included within the age group from 25 to 55 . They were excluded if they were terminal, had a current psychiatric diagnosis or significant cognitive deficites.

Instruments: The patients are asked with questionnaires $(n=20)$, five of 20 additionally with guided interviews. Guided interview is a tool of qualitative research to learn something about the opinion of the particular patient to gain an insight into the subject. They generate qualitative data. That's why the number of interviews usually is limited and quantitative conclusions cannot be drawn.

Results: The study is not finished yet but suggests that art therapy offered a personal, physical, and pictorial safe space. The first patients comments show that the structured intervention is helpful to stabilize the patients. An illustration of self and resources anchors the strengthening of self-awareness and self-effectiveness.

Disclosure: No conflict of interest disclosed.

\section{V982}

PsyCur - a curriculum for cancer patients

\section{Gaspar, M. $^{1}$}

${ }^{1}$ Hamm-Kliniken, Klinik Nordfriesland, Psychosozial, St. Peter-Ording,

Germany

The most common and therefore most typical affective response to a cancer diagnosis is fear. On the one hand, the diagnosis of cancer is always a confrontation with one's own mortality, on the other hand it creates strong uncertainty about one's future. This reflects the psycho-oncological specificity fear of recurrence. The future life is perceived as threatening, uncertain or uncontrollable. And that (of course) creates fear. Depathologizing of this affect helps to successfully deal with this "normal reaction". A program that has been proved successful in the inpatient cancer rehabilitation is a group approach. This program, developed here at the Klink Nordfriesland, is available for men with prostate carcinoma (Psycur P) in a three-part group concept, and for patients with other cancer diagnoses in a five-part group concept (Psycur). With a maximum of ten participants per group, the following issues are structurally discussed:

- Changes due to cancer (Psycur PsycurP)

- Dealing with the diagnosis (Psycur)

- Dealing with restrictions and strains (PsycurP)

- Dealing with fear and insecurity (PsycurP)

- Stress and stress management (Psycur)

- Dealing with anxiety and fear (Psycur)

- Health (salutogenesis) (Psycur)

Fear becomes understandable and explainable. Of course the fear is still present, however, it is often perceived as less threatening. Positive effects were seen not only in the short-term experience of patients during rehabilitation. Catamneses, which were conducted as part of the study "On the effectiveness of inpatient rehabilitation oncology with particular reference to specific oncological interventions" of the Institute of Medical Psychology at the UKEHamburg showed sustained stabilization of Psycur interventions. The study demonstrates the various elements of the curriculum and their specific characteristics. One of the main principles is not to pathologize fear. The lecture is intended to provide experiences, but also to serve as a basis for subsequent discussions and collegial exchange.

Disclosure: No conflict of interest disclosed.

\section{Wissenschaftliches Symposium Targets of cellular therapy in the context of allogeneic stem cell transplantation \\ V983 \\ Aspergillus fumigatus and Candida albicans shared T-cells epitopes drive the immune response in healthy individuals}

Topp, M.S. ${ }^{1}$

${ }^{1}$ Universitätsklinik Würzburg, Medizinische Klinik und Poliklinik II, Würzburg, Germany

Introduction: Invasive fungal infections (IFI) remains a leading cause of morbidity and infection-related mortality among patients undergoing hematopoietic stem cell transplantation (HSCT). IFI is caused mainly by $A$. fumigatus $(A F)$ and C.albicans $(C A)$. Although prophylaxis and treatment with antifungal drugs can efficiently reduce the incidence of IFI, breakthrough infections remain a major concern. As protective fungus-specific $T_{H} 1$ cells play a crucial role in the control of IFI and mice can be cross-protection with $C A$ against infection with $A F$, immunotherapeutic approaches may be promising but antigens with dual specifity have no yet been identified.

Methods: Recombinant fungal proteins, which demonstrated protection in mice were produced and a protein-specific T-cell response identified by ELISA in healthy donors. Immune dominant epitopes were identified with an overlap peptide library by ELISPOT assay. Capacity of T-cell clones to be activated by endogenous processed antigens was determined by DC feed with whole fungal lysats from different $A F$ and $C A$ strains. MHC-II restriction was 
identified with partial mismatched peptide pulsed B-LCL and the T-cell repertoire determined by identification of Vbeta usage. In order to quantify the frequency of $\mathrm{CD}^{+}{ }^{+} \mathrm{T}$-cells a MHC-II tetramer was synthesized.

Results: Amongst the 7 tested proteins, only Crf- 1 elicited a response in $>90 \%$ of donors and a minimal epitope was identified with a shared MHC II restriction for the 3 of the most common MHC class II alleles with in the Caucasian population. Epitope specific $\mathrm{CD}^{+} \mathrm{T}$-cell clones are efficiently activated by clinical strains of both $A F$ as well as $C A$ and demonstrate a broad Vbeta usage. The frequency of T-cell with dual specificity for both $A F$ and $C A$ is in range of 1:7000-1:10000 within the healthy $\mathrm{CD} 4^{+} \mathrm{T}$-cell population. In order to translate these results into a GMP compatible protocol, $\mathrm{CD}^{+} \mathrm{T}$ cells with dual specifity for both $A F$ and $C A$ can be readily expanded within 14 days with a cytokine combination of IL-2, IL-7 and IL- 15 to a purity of greater than $>50 \%$ without utilizing further selection procedures.

Conclusion: Epitopes which are generated by both A.fumigatus and C.albicans due to homology drive in healthy individuals the fungal immune response and induce a broad $\mathrm{T}_{\mathrm{H}} 1 \mathrm{CD}^{+} \mathrm{T}$-cell response across different MHC-allees. Adoptive transfer of $\mathrm{CD} 4^{+} \mathrm{T}$-cells with dual fungal specifity might be advantageous in patients at risk for development IF infections after HSCT.

Disclosure: Max S. Topp: Honoraria: Gliad

\section{V985 \\ NK cell therapy and ADCC as adoptive immunotherapy after SCT}

\section{Friedrichs, $B .^{1}$}

${ }^{1}$ Charite Universitätsmedizin Berlin - Campus Benjamin Franklin, Hämatologie/Onkologie/Transfusionsmedizin, Berlin, Germany

Human natural killer (NK) cells can discriminate between self- and non-selfMHC-class I molecules according to their repertoire of inhibitory killer Immunoglobulin receptors (KIR). NK cells can mediate alloreactions when faced with mismatched allogeneic targets sensing the missing expression of self-MHC class I alleles. This property plays an important role in haploidentical stem cell transplantation from NK alloreactive donors. Especially in acute myeloid leukemia (AML) NK cells can exert anti-leukemic immmune responses after haploidentical transplantation and adoptive NK cell transfer post transplant can consolidate leukemia control.As NK cells are known to promote lymphohematopoietic reconstitution without causing graft-versushost disease they can safely be used as NK cell donor lymphocyte infusions. Recent data, gives evidence to the hypothesis that only NK cells that can engage self-MHC become functionally competent or licensed. A difference might exist between licensed donor NK cells adoptively transferred and those NK cells regenerating from the bone marrow of the patients. In order to introduce NK cell immunotherapy into current treatment options different methods of NK cell expansion and NK cell generation.

The process of antibody-dependent cellular cytoxicity (ADCC) via the activating CD16 receptor is a second mechanism for elimination of tumor cells by NK cells.

ADCC can be enhanced by stimulation of NK cells with cytokines and concomitant use of expanded NK cells and monoclonal antibodies might be a successful combination, i.e. in the context of lymphoma. In-vitro assays suggest that immunomodulatory drugs (IMiDs) may lead to NK cell activation and also increase ADCC mediated by NK cells in response to different antibodies. The use of IMiDs and mABs may be a synergistic treatment option in combination with adoptive NK cell transfer.

Disclosure: No conflict of interest disclosed.

\section{V986}

Impact of DLI on long term outcome in patients with acute leukaemia

\section{Schmid, C.}

${ }^{1}$ Klinikum Augsburg, Med. Klinik II, Augsburg, Germany

Donor lymphocyte infusion (DLI) for treatment of leukaemia relapse after allogeneic stem cell transplantation (SCT) has been a milestone in the field of immunotherapy against malignant disease. It can be regarded as the proof of principle for the Graft-versus-Leukemia effect.

In contrast to CML and early stages of MDS, DLI was less effective in the treatment of post SCT relapse in highly proliferative diseases as acute leukaemia. Rapid pace of the disease, and reduced immunogenicity of the malignant blasts are regarded the main reasons for the inferior response. Nevertheless, strategies including DLI were more effective in the treatment of AML relapse after SCT than treatment without the use of donor cells. When given as maintenance therapy after effective cytoreduction, DLI could induce long term remissions, both after standard and reduced intensity conditioning transplants. These data have prompted the strategy to use donor cells even before the occurrence of overt haematological relapse, i.e in minimal residual disease, mixed or falling donor chimerism, or even prophylactically. This strategy is now widely used across European transplant centres and has shown promising results. Future strategies include concepts to increase the cytotoxic capacity of donor cells, the use of specific cell subsets, or the combination of targeted therapies and donor cell based strategies.

Disclosure: No conflict of interest disclosed.

\section{V987 \\ Induction of graft-versus-leukemia (GvL) effects by specific $T$ cell therapy}

Herr, $W^{1}$

${ }^{1}$ Universitätsmedizin der Johannes Gutenberg-Universität, 3. Medizinische Klinik, Hämatologie und Onkologie, Mainz, Germany

Introduction: The main therapeutic principle of allogeneic hematopoietic stem cell transplantation (aHSCT) is to replace the failed antitumor immunosurveillance in leukemia patients by the non-tolerized immune system of HLA-compatible healthy donors. Unfortunately, this "exchange" is frequently associated with misdirected and insufficient donor-derived immune functions, clinically resulting in graft-versus-host disease, opportunistic infections and leukemia relapse.

Methods and results: Our group intends to overcome these limitations by the adoptive transfer of leukemia-reactive and pathogen-reactive $\mathrm{T}$ cells generated from donor lymphocytes by primary in vitro stimulation and $\mathrm{T}$ cell receptor gene transfer. As a source for producing leukemia-reactive CD8+ cytotoxic $\mathrm{T}$ lymphocytes (CTL) we use the CD45RA+ naive CD8+ T cell subset of healthy donors because of the superior growth potential and early $\mathrm{T}$ cell differentiation phenotype of naive-derived effector cells, which translates into improved expansion and homing capacities in vivo. We also show that CD45RA-derived leukemia-reactive CD8+ CTL recognize minor histocompatibility and leukemia-associated antigens and are capable of preventing the engraftment of human primary leukemia blasts in immunodeficient mice.

Conclusions: Our strategy based on the use of naive-derived CD8+ leukemiareactive CTL rather than entire donor lymphocyte preparations should help to optimize the risk-benefit ratio of aHSCT. The intended clinical translation of the procedure will show whether the general nihilism concerning the successful separation of beneficial from detrimental immune functions in donor lymphocytes is appropriate and whether specific allogeneic T cell therapy in the context of aHSCT can become a reality.

Disclosure: No conflict of interest disclosed.

v988

\section{Chimerism triggered cellular intervention after allogeneic} SCT

Bornhäuser, M. ${ }^{1}$; Platzbecker, U. ${ }^{1}$; Ehninger, G. ${ }^{1}$; Thiede, C. ${ }^{1}$ ${ }^{1}$ Med. Klinik und Poliklinik I, Hämatologie/Onkologie, Dresden, Germany

In high risk malignant diseases like acute myeloid leukemia (AML), myelodysplastic syndrome (MDS) or acute lymphoblastic leukemia (ALL), relapse remains the major cause of treatment failure after allogeneic stem cell transplantation (SCT). Donor lymphocyte infusions and second allogeneic transplantation have a limited efficacy in patients after overt hematologic relapse. Methods to improve the sensitivity of chimerism analyses include the combination of fluorescence activated cell sorting of specific cellular subpopulations 
and subsequent chimerism analysis or the use of fluorescence in-situ hybridization and immunocytochemistry (FICTION). Several groups have applied chimerism analysis in cells expressing the leukemic phenotype, e.g. cells expressing aberrant antigens. CD34 is expressed on blast cells of more than $80 \%$ of all cases of AML and more than $50 \%$ of all ALL patients. However, sufficient numbers of CD34+ cells for chimerism analysis derived from bone marrow aspirates may be difficult to obtain at short intervals. The sensitivity of conventional chimerism analyses can be significantly increased when using purified CD34+ cells in patients in whom the original disease expressed CD34. This quantitative assay for chimerism analysis can be performed out of peripheral blood and requires low cell numbers.

In a prospective study, we could demonstrate that this method allows to prepone the detection of relapse by two months. This theoretically opens a window for cellular therapies like donor lymphocyte infusion (DLI). Recent data suggest that a combination of cytoreductive strategies like 5-azacyticine and DLI might be even more effective in high-risk patients with AML or MDS who show a decrease in CD34+ chimerism. In sex-mismatched settings a Y-chromosome specific chimerism analysis might even be more sensitive to trigger DLI or other cellular therapies.

In summary, the development of novel sensitive techniques to detect decreasing donor chimerism during the later course after allogeneic SCT has allowed to increase the feasibility and efficacy of adoptive cellular immunotherapy in high-risk leukemia patients after allogeneic SCT. Novel strategies combining pharmacological with immunological therapies are currently tested in clinical trials

Disclosure: Martin Bornhäuser: No conflict of interest disclosed.

Christian Thiede: Employment or Leadership Position: Angestellt/Leitende Funktion bei Fa Agendix

\section{Arbeitskreis Sitzung \\ Personalisierte Medizin und Patientenorientierung: Ethische und klinische Aspekte der Therapieentscheidung in der Hämatologie und Onkologie \\ V990 \\ Chances and challenges of shared decision making: An empirical ethics study about decisions to limit treatments}

\section{Winkler, E.}

${ }^{1}$ University Hospital Grosshadern, Ludwig-Maximilians University, Dept. of Internal Medicine III, Munich, Germany

Purpose: This session is dedicated to ethical questions around personalized medical treatment and patient oriented care. It is organized by the "Medicine and Ethics" Working group of the DGHO.

Methods: A. Ganser, Hannover will discuss clinical and ethical challenge in implementing molecular diagnostics in therapeutic decisions for patients with Acute Myeloic Leukemia. Decisions to limit life-prolonging treatment (DLT) precede up to two thirds of all non-sudden deaths in European countries. The actual role of patients in decisions to limit treatment and ethical difficulties of shared decision making at the end-of-life is discussed by E.Winkler, Munich. M.Feufel, Berlin presents different strategies of communicating risks, statistical probabilities and prognosis for patients who have personalized treatment options. M.Hallek, Köln (not yet confirmed) will discuss what kind of evidence is needed for new therapeutics to be become "standard" therapy in clinical oncology.

Results: Molecular risk stratification and therapy is gaining importance in therapeutic decisions in oncology and hematology. In contrast to the biomedical implications, the ethical and practical relevance has not yet been addressed. This session aims to highlight the ethical and practical implications of personalized medicine.

Disclosure: No conflict of interest disclosed.

\section{Freie Vorträge \\ Experimentelle Onkologie \\ V992 \\ Suppression of VEGFR2 expression in human endothelial cells by Dimethylfumarate treatment: First evidence for anti-angiogenic action}

Meissner, M. ${ }^{1}$; Stein, M. ${ }^{1}$; Hrgovic, I. ${ }^{1}$; Reichenbach, G. ${ }^{1}$; Gille, J. ${ }^{1}$; Kaufmann, $R^{1}{ }^{1}$

${ }^{1}$ Universitätsklinik Frankfurt, Zentrum für Dermatologie und Venerologie,

Frankfurt, Germany

The association between angiogenesis and tumor development seems to be an important phenomenon implicated in the pathogenesis of these medical conditions. Recently, it could be demonstrated, that Dimethylfumarate, an oral available anti-psoriatic drug, reduces melanoma metastasis in a SCID mouse model. As signaling via the vascular endothelial growth factor receptor-2 (VEGFR2) pathway is critical for angiogenic responses, we explored whether dimethylfumarate exhibits antiangiogenic effects mediated by diminished VEGFR2 expression.

In this study, Dimethylfumarate (DMF) is found to inhibit endothelial VEGFR2 expression. Time- and concentration-dependent inhibition is demonstrated both at the level of protein and mRNA VEGFR2 expression. This blockade was paralleled by the respective inhibition of the formation of capillary-like structures and endothelial cell migration. In contrast, neither membrane bound neuropilin-1 nor VE-Cadherin expression was significantly affected by DMF treatment. The suppressive effects on VEGFR2 expression were not conveyed by increased shedding or by a decrease in the protein halflife, suggesting that transcriptional mechanisms accounted for the observed effects. Inhibitory effects of DMF on transcriptional activity of the VEGFR2 promoter are conveyed by an element located between base pairs -60 and -37 that contains two adjacent consensus Sp1 transcription factor binding sites. Constitutive Sp1-containing complex formation to this sequence is decreased by DMF treatment, indicating that VEGFR2 gene expression is inhibited by repressing Sp1 site-dependent DNA binding and transactivation. Additional data demonstrates that binding is lost because phosphorylation of $\mathrm{Sp} 1$ at Thr453 is inhibited. Hence, VEGFR-2 constitutes a critical molecular target of DMF that may mediate anti-angiogenic effects in vivo, demonstrating a new mechanism of action.

Disclosure: No conflict of interest disclosed.

\section{V993}

Peroxisome proliferator-activated receptors regulate the expression of cathepsin B in human endothelial cells in a transcriptional and post-transcriptional manner

Reichenbach, G. ${ }^{1}$; Stein, M. ${ }^{1}$; Gille, J. ${ }^{1}$; Kaufmann, R. ${ }^{1}$; Meissner, $M .^{1}$

${ }^{1}$ Universitätsklinik Frankfurt, Zentrum für Dermatologie und Venerologie, Frankfurt, Germany

Malignant tumors recruit vasculature and stromal cells through production and secretion of stimulatory growth factors and cytokines to activate their local host tumor microenvironment. In this context, the reorganization of the extracelluar matrix is an important tumor initiated process. A group of lysosomal proteases, the cathepsins, especially Cathepsin B and L, were described to be crucial in the process of metastasis and angiogenesis. Peroxisome proliferatoractivated receptors (PPARs) are ligand-activated transcription factors, mainly implicated in the regulation of lipid and glucose homeostasis. In addition, PPAR agonists have also shown to function as anti-inflammatory and antiangiogenic molecules. We therefore explored the effect of PPAR $\alpha$ and PPAR $\delta$ agonists on Cathepsin B expression by endothelial cells. Both inhibited the endothelial Cathepsin B protein expression in a time and dose dependent manner. We further investigated whether the inhibition in Cathepsin B protein synthesis is mediated by changes in the mRNA expression level. Interestingly, treatment with PPAR $\alpha$ agonist considerably suppressed Cathepsin B mRNA accumulation, whereas PPAR $\delta$ ligands failed to do so. Analysis of 5'-deletional Cathepsin B promoter-based constructs revealed, that PPAR $\alpha$ ligands 
mediate their inhibitory effects through an E-box-binding site. Finally, EMSA analysis demonstrated that PPAR $\alpha$ activators reduce the binding of the heterodimer USF1/USF2 at this E-box located between the base pairs -16 and +17 at the Cathepsin B promoter sequence.

In further analysis we could demonstrate that PPAR $\delta$ agonists conveyed their suppressive effects by a shortened Cathpsin B protein half-life. As the translational efficiency of Cathepsin B mRNA can depend in part on their $5^{\prime}$ and 3 untranslated regions (UTRs), we hypothesized that the inhibitory effect of PPAR $\delta$ ligands on Cathepsin B expression might be mediated via an UTR dependent mechanism. First transcriptional activation studies with Cathepsin B UTR reporter gene constructs demonstrated that PPAR $\delta$ ligands do no convey their inhibitory effects on Cathepsin B expression by the 5'UTR Further 3'UTR reporter gene studies are now under investigation. In conclusion, our data identify for the first time endothelial Cathepsin B expression as a novel target for PPAR $\alpha$ and PPAR $\delta$ agonist. As antivascular effects of PPARs are increasingly appreciated, enhanced insights in their modes of action are critical to define new therapeutic drugs.

Disclosure: No conflict of interest disclosed.

\section{V994}

The p14ARF tumorsuppressor triggers p53-independent apoptosis by preferential down-regulation of the antiapoptotic $\mathrm{Bcl}-2 \mathrm{Homolog} \mathrm{Mcl}-1$ and subsequent activation of pro-apoptotic Bak

Müer, A. ${ }^{1,2}$; Overkamp, T.1,2; Gillissen, B. ${ }^{1,2}$; Dörken, B. ${ }^{3}$; Daniel, P. ${ }^{1,2,3}$; Hemmati, $P .{ }^{1,2,3}$

${ }^{1}$ Charité - Universitätsmedizin Berlin, Experimental and Clinical Research Center, Berlin, Germany, ${ }^{2}$ Max-Delbrück-Center for Molecular Medicine, Clinical and Molecular Oncology, Berlin, Germany, ${ }^{3}$ Charité -

Universitätsmedizin Berlin, Medizinische Klinik m.S. Hämatologie und Onkologie, Berlin, Germany

Introduction: The p14ARF tumor suppressor plays an essential role in mediating apoptosis, cell cycle arrest, and senescence following the activation of various cellular stress signals. We previously showed that the expression of exogenous p14ARF induces apoptosis irrespective of the presence or absence of functional p53 or the pro-apoptotic Bcl-2 family member Bax. However, the mechanism of p14ARF-indcued apoptosis in p53/Bax-deficient cells remained unclear.

Methods and results: Here, we show that expression of p14ARF in p53deficient DU145 prostate cancer cells, which are void of Bax but still express the pro-apoptotic Bax-homolog Bak, triggers the mitochondrial apoptosis pathway. This was evidenced by the breakdown of the mitochondrial membrane potential and the release of cytochrome $\mathrm{c}$ followed by the activation of caspases and subsequent apoptotic DNA-fragmentation. Whereas re-expression of pro-apoptotic Bax had no effect, over-expression of pro-apoptotic Bak strongly sensitized for p14ARF-induced apoptosis. Furthermore, the preferential activation of Bak during p14ARF-induced mitochondrial activation was demonstrated by the detection of an N-terminal conformational change and clustering of Bak, rather than Bax, upon expression of p14ARF. Furthermore, expression of p14ARF correlated with the strong down-regulation of antiapoptotic Mcl-1 at the post-transcriptional level. Importantly, secondary cleavage of Mcl-1 via caspases was ruled out by addition of the pan caspase inhibitor Q-VD-Oph. Furthermore, we observed the up-regulation of proapoptotic of $\mathrm{BH} 3$-only proteins, i.e. Noxa, Puma and Nbk, following expression of p14ARF.

Conclusions: Taken together, these data indicate that in the absence of functional p53 p14ARF triggers the mitochondrial apoptosis pathway by activating Bak, rather than Bax, via the differential activation of specific pro- and antiapoptotic Bcl-2 family proteins. This may represent an important fail-safe mechanism allowing the elimination of p53/Bax-deficient tumor cells following cellular stress signals such as oncogene activation of DNA damage.

Disclosure: No conflict of interest disclosed.
V995

Lack of association between polymorphisms of AKT1, AKT2 and FRAP1 and outcome in advanced renal cancer treated with sorafenib

Pfisterer, K. ${ }^{1}$; Fusi, A. ${ }^{1}$; Busse, A. ${ }^{1}$; Zimmermann, K. ${ }^{1}$; Nonnenmacher, A. ${ }^{1}$; Keilholz, U. ${ }^{1}$

${ }^{1}$ Charité - Campus Benjamin Franklin, Hämatologie und Onkologie, Berlin, Germany

Purpose: The PI3K/PTEN/AKT/mTOR signaling pathway is responsible for balancing survival and apoptosis in cancer cells and it has been implicated in resistance to several chemotherapeutic agents. The aim of this study was to investigate whether single nucleotide polymorphisms (SNPs) in AKT1, AKT2 and FRAP1 (encoding mTOR) were associated with the tumor response and survival in advanced renal cancer patients (pts) who received sorafenib.

Methods: Six single SNPs in AKT1 (rs3803304, rs2494738), AKT2 (rs892119, rs8100018) and FRAP1 (rs892119, rs2295080) were genotyped by means of Real Time PCR system and analysed for association with response to therapy and survival.

Results: Forty-nine pts (32 male, 17 female) were evaluated (median age: 64 yrs [range: 37-78 yrs]; MSKCC score: favourable 10; intermediate 34; poor 5). All the pts were evaluable for response. The median follow up was 16 months [1-28 months]. None of the SNPs were found to be associated with risk of progression or survival. Similarly we did not observe any association between SNPs and response to therapy.

Conclusions: The PI3K/PTEN/AKT/mTOR signaling pathway does not influence clinical outcomes in pts with advanced renal cancer who undergo treatment with sorafenib.

Disclosure: No conflict of interest disclosed.

\section{V996}

Differential impact of the endogenous Bak inhibitors Mcl-1 and $\mathrm{BCl}-\mathrm{x}_{\mathrm{L}}$ on death receptor-induced apoptosis

Gillissen, B. ${ }^{1,2}$; Wendt, J. ${ }^{1}$; Hemmati, P. G. ${ }^{1,3}$; Overkamp, T. ${ }^{1}$;

Richter, A. ${ }^{1}$; Müer, A. ${ }^{1}$; Milojkovic, A. ${ }^{1}$; Preissner, R. ${ }^{1}$; Belka, C. ${ }^{4}$; Dörken, B. ${ }^{1,3}$; Daniel, P.T. ${ }^{1,2,3}$

${ }^{1}$ Charité - Universitätsmedizin Berlin, Experimental and Clinical Research Center, Berlin, Germany, ${ }^{2}$ Max-Delbrück-Center for Molecular Medicine, Clinical and Molecular Oncology, Berlin, Germany, ${ }^{3}$ Charité -

Universitätsmedizin Berlin, Medizinische Klinik m.S. Hämatologie und Onkologie, Berlin, Germany, ${ }^{4}$ Maximilians Universität, Klinik und Poliklinik für Strahlentherapie und Radioonkologie, München, Germany

TRAIL (TNF-related apoptosis-inducing ligand), a cytotoxic ligand of the tumor necrosis factor (TNF) family, is a promising anticancer agent. TRAIL induces cell death in a wide range of human cancers independently of their p53 status without apparent toxic side effects in normal tissues. In the majority of tumors the death receptor signal requires amplification via the mitochondrial apoptosis pathway, which is mainly regulated by proteins of the $\mathrm{Bcl}-2$ family. In these so-called ,type II cells” TRAIL-induced cell death entirely depends on the presence of Bax, which is a proapoptotic member of the Bcl-2 family. However, Bax is often lost due to epigenetic inactivation or, in mismatch repair-deficient cancers, due to mutations. Despite expression of the Bax homolog Bak, Bax deficiency confers resistance of tumor cells to TRAILinduced apoptosis.

Characterization of TRAIL-induced cell death revealed that Bax deficient cells are resistant to TRAIL because TRAIL fails to activate the Bax homolog Bak. Two anti-apoptotic Bcl-2 family members, Mcl-1 and Bcl-xL, keep Bak in check. Blocking these Bak inhibitors showed that only inactivation of Mcl-1 overcomes TRAIL resistance by enabling TRAIL to activate Bak. Interestingly, this also proves true for TNF $\alpha$ - and CD95/FasL-induced apoptosis confirming a differential impact of the endogenous Bak inhibitors Mcl-1 and Bcl-xL on death receptor-induced apoptosis in Bax-deficient carcinoma cells. Given the therapeutic impact of our finding on resistance to TRAIL receptor ligation, we also have shown that small molecules, known to down-regulate Mcl-1 expression, can overcome TRAIL resistance. The multikinase inhibitor sorafenib and the cyclin-dependent kinase inhibitor roscovitine both sensitized and efficiently overcame TRAIL resistance of cells mutated for Bax. Interestingly, 
loss of Bax is a frequent event in human cancer, whereas Bak expression persists in most cancers. Thus, down-regulation or blocking of endogenous Bak inhibitors like Mcl-1 by small molecules appears to be a promising strategy to overcome restraints in apoptosis signalling as a result of Bax deficiency and to sensitize these tumors for TRAIL- induced apoptosis via a Bak-dependent pathway.

Disclosure: No conflict of interest disclosed.

\section{V997}

Characterization of EGFR expression in circulating tumor cells in SCCHN

Hristozova, T.1; Konschak, R. ${ }^{1}$; Fusi, A. ${ }^{2}$; Grün, A. ${ }^{1}$; Budach, V. ${ }^{1}$; Keilholz, U. ${ }^{2}$; Tinhofer, ${ }^{1}{ }^{1}$

${ }^{1}$ Charité Universitätsmedizin Berlin, Klinik für Strahlentherapie, Berlin, Germany, ${ }^{2}$ Charite CBF, Department of Hematology and Oncology, Berlin, Germany

Introduction: Our recent analysis of circulating tumor cells (CTCs) in the peripheral blood of SCCHN patients revealed their presence in about $40 \%$ of cases. It has been previously shown that overexpression of epidermal growth factor receptor (EGFR) is not only significantly involved in tumorigenesis and the progression of the primary tumor but also in the development of metastatic disease. In this study we therefore assessed the frequency of EGFR expression in CTCs in peripheral blood of SCCHN patients.

Methods: After informed patient consent, peripheral blood from 65 SCCHN patients was collected for flow cytometric characterization of EGFR in CTCs. For 15 samples both flow cytometry and nested RT-PCR were applied for detection of EGFR. For flow cytometry erythrocytes were lysed and samples were enriched for CTCs by immunomagnetic depletion of CD45+ leukocytes. Samples were stained with anti-CD45 antibody for the discrimination between tumor and white blood cells, and with antibodies to the specific epithelial markers EpCAM and cytokeratin and to EGFR. Unspecific antibody binding was assessed by staining with the relevant isotype control antibodies. Absolute numbers of EpCAM+cytokeratin+CD45- CTCs in $3.75 \mathrm{ml}$ blood and their EGFR expression profile were determined.

For detection of EGFR transcripts in non-enriched peripheral blood samples a nested RT-PCR was established. Detection of EGFR was performed for each sample in triplicates. GAPDH was used as a control.

Results: Using flow cytometry we detected CTCs in 19 out of 65 SCCHN cases $(32 \%)$. In these CTC+ cases EGFR was expressed in $86 \%$ of samples. Our comparative analysis of flow cytometry and nested RT-PCR for detection of EGFR+ CTCs in the subgroup of 15 patients revealed good concordance for both methods (chi-square: $\mathrm{p}=.06$ ): EGFR+ CTCs were detected by flow cytometry in 6 out of 15 samples and in 5 of these cases $(83 \%)$ EGFR transcripts were also detected by the PCR approach. Furthermore, in the 9 out of 15 samples for which flow cytometry revealed absence of CTCs or presence of CTCs without EGFR expression the EGFR PCR analysis was also negative in $78 \%$.

Conclusions: The evaluation of EGFR expression in CTCs by flow cytometry or nested RT-PCR might help in identifying patients likely to benefit from anti-EGFR treatment of metastatic disease.

Disclosure: No conflict of interest disclosed.

\section{Plenarsitzung \\ Presidential Symposium Behring Lecture / P. Ehrlich Lecture \\ V998 \\ Human bone marrow failure and the role of telomere biology in human disease}

\section{Young, N. $^{1}$}

${ }^{1}$ Hematology Branch, National Heart, Lung, and Blood Institute, Bethesda, United States

Telomeres, the natural ends of linear chromosomes, are composed of repetitive hexanucleotides and the shelterin proteins. Telomere attrition is attenuated by telomerase, a complex of an RNA template (TERC), a reverse transcriptase (TERT), and associated proteins. Very short telomeres of peripheral blood leukocytes occurs in acquired aplastic anemia. A genetic explanation was provided by identification through linkage analysis of families with dyskeratosis congenita: $D K C 1$, which encodes a protein that stabilizes telomerase, was mutant in $\mathrm{X}$-linked pedigrees. Mutations in telomerase and shelterin genes are determinative in dyskeratosis and risk factors in aplastic anemia. Telomerase mutations decrease enzymatic activity and lead to accelerated telomere attrition. They are etiologic in pulmonary fibrosis and hepatic cirrhosis as well as aplastic anemia. Telomerase deficiency is also at the nexus of malignant transformation from inflammatory states. TERT mutations are present in acute myeloid leukemia. In acquired aplastic anemia, short telomeres early in disease (absent known telomerase mutations), strongly predict later clonal evolution to myelodysplasia and leukemia. Telomeres have been implicated in oncogenesis (Barrett's esophagus and inflammatory bowel disease). While critically short telomeres usually signal cell senescence or apoptosis, they can lead to chromosome instability and aneuploidy, directly demonstrable in human bone marrow cultures. Sex hormones modulate TERT and up-regulate telomerase, providing a mechanism for activity in marrow failure and also potential therapy of telomere attrition.

Disclosure: No conflict of interest disclosed. 